\title{
Digital technology-enabled home health care
}

\author{
Citation for published version (APA):
}

van de Weijer, S. C. F. (2021). Digital technology-enabled home health care: gamification in online cognitive therapies for Parkinson's disease. [Doctoral Thesis, Maastricht University]. Gildeprint Drukkerijen. https://doi.org/10.26481/dis.20210901sw

Document status and date:

Published: 01/01/2021

DOI:

10.26481/dis.20210901sw

Document Version:

Publisher's PDF, also known as Version of record

\section{Please check the document version of this publication:}

- A submitted manuscript is the version of the article upon submission and before peer-review. There can be important differences between the submitted version and the official published version of record.

People interested in the research are advised to contact the author for the final version of the publication, or visit the DOI to the publisher's website.

- The final author version and the galley proof are versions of the publication after peer review.

- The final published version features the final layout of the paper including the volume, issue and page numbers.

Link to publication

\footnotetext{
General rights rights.

- You may freely distribute the URL identifying the publication in the public portal. please follow below link for the End User Agreement:

www.umlib.nl/taverne-license

Take down policy

If you believe that this document breaches copyright please contact us at:

repository@maastrichtuniversity.nl

providing details and we will investigate your claim.
}

Copyright and moral rights for the publications made accessible in the public portal are retained by the authors and/or other copyright owners and it is a condition of accessing publications that users recognise and abide by the legal requirements associated with these

- Users may download and print one copy of any publication from the public portal for the purpose of private study or research.

- You may not further distribute the material or use it for any profit-making activity or commercial gain

If the publication is distributed under the terms of Article $25 \mathrm{fa}$ of the Dutch Copyright Act, indicated by the "Taverne" license above, 
Digital technology-enabled home health care

Gamification in online cognitive therapies for Parkinson's disease

Sjors van de Weijer 
All rights reserved. No parts of this publication may be reproduced or transmitted in any form or by any means, electronic or mechanical, including photocopy, recording, or any information storage or retrieval system, without permission in writing from the author. The copyright of the articles that have been accepted for publication or that have already been published, has been transferred to the respective journals.

Cover design and illustrations: Lienke Raben, lienkeraben.com

Thesis layout: Anna Bleeker, persoonlijkproefschrift.nl

Printing: Gildeprint Enschede, gildeprint.nl

ISBN: 978-94-6423-385-8

The research presented in this work was partly supported through a grant received from MyCognition Ltd.

Copyright $(2021$ Sjors C.F. van de Weijer 


\section{Digital technology-enabled home health care Gamification in online cognitive therapies for Parkinson's disease}

\section{PROEFSCHRIFT}

ter verkrijging van de graad van doctor aan de Universiteit Maastricht, op gezag van de Rector Magnificus, Prof. dr. Rianne M. Letschert, volgens het besluit van het College van Decanen,

in het openbaar te verdedigen

op woensdag 1 september 2021 om 13:00 uur

door

Sjors Camille Frans van de Weijer

geboren op 12 februari 1988 te Maastricht 


\section{Promotor}

Prof. dr. B.R. Bloem

Radboudumc Nijmegen

\section{Copromotoren}

Dr. A.A. Duits

Dr. M.L. Kuijf

Dr. N.M. de Vries

Radboudumc Nijmegen

\section{Beoordelingscommissie}

Prof. dr. S.A. Kotz (voorzitter)

Prof. dr. C.M. van Heugten

Prof. dr. L. Fasotti

Radboud Universiteit Nijmegen

Dr. R. Esselink

Radboudumc Nijmegen 
Ter nagedachtenis aan Keiron Sparrowhawk For his inspiring entrepreneurship in health care and technology 



\section{TABLE OF CONTENTS}

$\begin{array}{lc}\text { Abbreviations } & 8\end{array}$

$\begin{array}{ll}\text { Chapter 1: General introduction } & 12\end{array}$

Chapter 2: Resting-state fMRI in Parkinson's disease patients with cognitive $\quad 22$ impairment: A meta-analysis

Chapter 3: Promising non-pharmacological therapies in PD: Targeting late stage $\quad 58$ disease and the role of computer based cognitive training

Chapter 4: Do-It-Yourself Gamified Cognitive Training: A Viewpoint 72

Chapter 5: The validation of a new online cognitive assessment tool: 88 The MyCognition Quotient

Chapter 6: The Parkin'Play study: protocol of a phase II randomized controlled trial to assess the effects of a health game on cognition in Parkinson's disease

Chapter 7: Feasibility of gamified cognitive training in Parkinson's disease: the randomized controlled Parkin'Play pilot

Chapter 8: Summary, General Discussion, and Potential Impact

Discussion \& interpretation

Potential impact of this thesis

Dankwoord

About the author 


\section{ABBREVIATIONS}

AES-SDM

CANTAB

CT

DMN

$H \& Y$

LEDD

MMSE

$\mathrm{MoCa}$

$\mathrm{MyCQ}^{\mathrm{TM}}$

PD

PDD

PD-Cl

$\mathrm{PD}-\mathrm{MCl}$

$\mathrm{MCl}$

MRI

MUMC+

RCT

UPDRS
Anisotropic effect size of signed differential mapping

Cambridge Neuropsychological Automated Test Battery

Cognitive training

Default mode network

Hoehn \& Yahr scale

Levodopa equivalent daily dosage

Mini Mental State Exam

Montreal Cognitive Assessment

MyCognition Quotient

Parkinson's disease

Parkinson's disease dementia

Cognitively impaired Parkinson's disease

Parkinson's disease with Mild cognitive impairment

Mild Cognitive Impairment

functional magnetic resonance imaging

Maastricht University Medical Center

Randomized Controlled Trial

Unified Parkinson's Disease Rating Scale 
9 


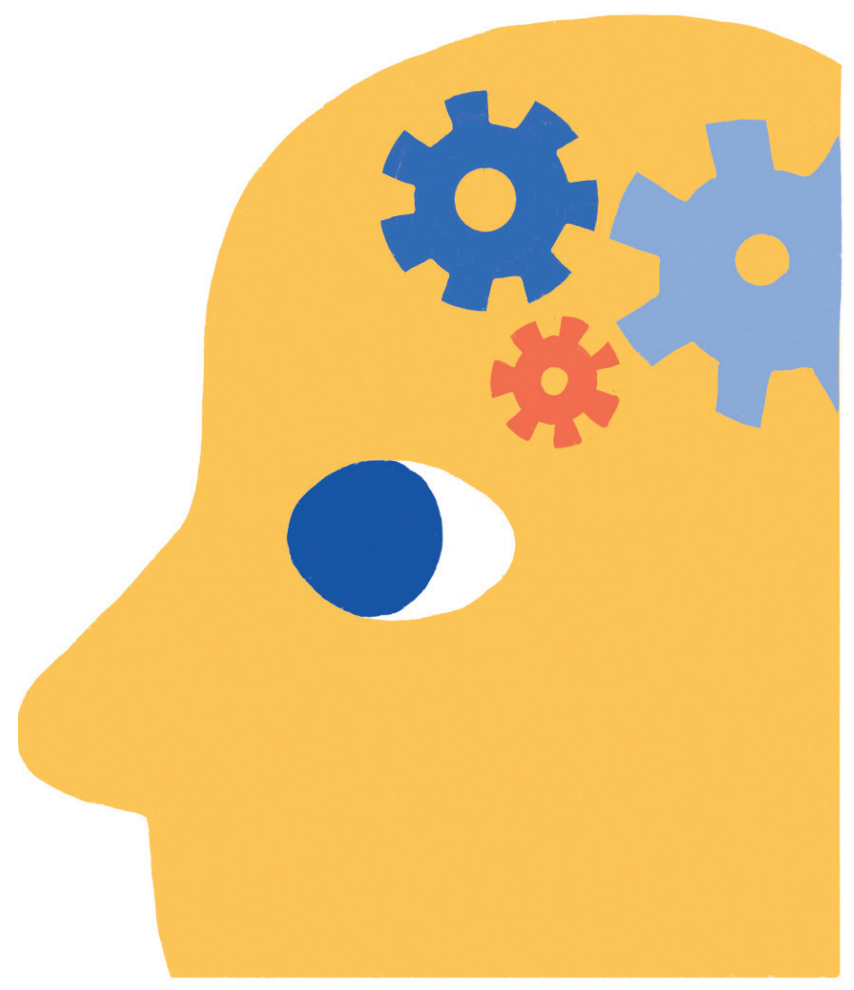




\section{CHAPTER 1}

\section{General introduction}

Parts of this chapter are based on: Van de Weijer SCF, Duits AA, Bloem BR, Kessels RP, Jansen JFA, Köhler S, Tissingh G, Kuijf ML.

The Parkin'Play study: protocol of a phase II randomized controlled trial to assess the effects of a health game on cognition in Parkinson's disease. BMC Neurol BioMed Central; 2016 Nov 3;16(1):209. PMID: 27809791 


\section{PARKINSON'S DISEASE}

Parkinson's disease (PD) is a neurodegenerative disorder that globally affects more than 6 million people; this number is expected to double in the next 20 years [1]. Several factors play a role in the development of the clinical manifestations of PD, but the main pathological factor is a loss of dopamine producing cells in the substantia nigra. Dopamine is a neurotransmitter that plays an large role in controlling and coordinating body movements and other automatic processes, as well as in regulating mood and a variety of cognitive processes. PD is traditionally characterized by motor symptoms, such as a rigidity, resting tremor, and bradykinesia, which start gradually with sometimes barely noticeable unilateral symptoms. As the disease worsens over time, the symptoms progress and extend to bilateral and more severe disabilities. Importantly, PD patients are not solely affected by these readily discernible motor symptoms, but the disease is also characterized by a wide range of non-motor symptoms. This includes autonomic features, pain, sleep disorders and various neuropsychiatric symptoms. Frequently occurring examples of these neuropsychiatric symptoms include depression, anxiety, apathy and psychosis. Both the motor and the non-motor symptoms contribute to the mounting disability experienced by patients with clinically overt PD. A more recent insight is that both the motor and the non-motor symptoms can be present very early on in the disease course, even prior to the formal diagnosis of PD.

\section{COGNITION AND COGNITIVE DECLINE}

Cognitive decline is one of those frequently occurring non-motor symptom in PD and is recognized as a major contributor to a decreased quality of life in PD.

Cognition is an umbrella term for intellectual thinking processes, such as memory, decision-making, attention, reasoning, etc., involved in acquiring knowledge and understanding (new) information.

Cognitive impairment is present in a substantial proportion of the PD population. Mild cognitive impairment is the term used for the transition between normal cognitive aging and serious cognitive decline interfering with daily life (dementia) [2]. Mild cognitive impairment in Parkinson's disease (PD-MCI) is present in up to $20-40 \%$ - depending on the dataset - of newly diagnosed patients [3]. More pronounced decline, including dementia, can ultimately be seen in up to $83 \%$ of PD patients [4]. Common cognitive deficits in PD include impairment in cognitive flexibility, reasoning, attention, planning, and verbal processes [5]. Mild cognitive impairment is associated with a decreased quality of life, an increased caregiver burden and an elevated risk of developing dementia [6]. In order to improve or delay cognitive decline and perhaps even bring it to a halt, many scientists have recently focused on developing improved or new treatment options to alleviate cognitive symptoms for both the general population and for patients with PD.

In some patients, cognitive symptoms remain stable over years before progressing to dementia, whereas in others the disease sets in at a faster pace. This suggest that, from a 
biological perspective, the pathophysiology of cognitive decline may be complex. Current evidence points to several potential changes in the brain, among which the most prominent are the accumulation of beta-amyloid protein plaques between brain nerve cells, the formation of neurofibrillary tau tangles in brain cells, or the build-up of Lewy bodies [7]. The presence of plaques and tangles in and around brain cells can trigger an immune response and cause cell death. Lewy bodies are unusual clumps of alpha-synuclein inside brain neurons that are associated with a decreased activity of neurons, and, eventually, succumbed neural activity. These biological changes can be found in the cortex in areas involved in cognition using advanced autopsy studies, but are currently impossible to diagnose in a living human being. A diagnosis of cognitive decline or dementia is established based on clinical grounds, including a neurological examination that involves a comprehensive testing of mental abilities (cognitive assessment). Some of these methods rule out possible other conditions that cause similar symptoms, such as a concurrent depression.

Brain scans such as MRI, CT or PET scans are ancillary tests that assist in the diagnosis and understanding of cognitive decline. An interesting neuroimaging method to assess cognitive decline is fMRI (functional MRI), a technique that exploits the link between cerebral blood flow and neuronal activation. fMRI measures functional brain activity changes, as a result of cognitive activation, by detecting temporal changes in blood flow. The progressive cognitive decline is paralleled by functional changes in the brain, but it is largely unknown what changes specifically cause the degenerative process of PD-MCI [8]. Resting-state fMRI is a relatively new method utilized to decipher the cause of cognitive decline. Using this method, regional interactions in the brain are evaluated that occur during rest. Resting-state fMRI is used to assess functional brain connectivity, which is the connectivity between brain regions that share functional features.

In patients manifesting cognitive decline, several functional connectivity changes occur between selective brain regions. Global pattern changes of resting-state functional connectivity have been associated with widespread connectivity decrements in several networks in the brain, including the default-mode network (DMN) and occipital networks $[3,9,10]$. The DMN is a network in the brain that is thought to serve an important role in several higher order cognitive functions, such as autobiographical memory and envisioning the future. So far, the underlying pathophysiology of cognitive decline remains unclear. Neuroimaging studies contribute to the understanding of the pathophysiology of cognitive decline in Parkinson patients and functional connectivity alterations may become a promising future biomarker.

\section{COGNITIVE TRAINING}

Unfortunately, few treatment options are available to treat cognitive decline. A pharmacological treatment with very limited effectiveness is rivastigmine: an acetylcholinesterase inhibitor that inhibits the acetylcholinesterase enzyme from breaking down acetylcholine into choline and acetate [11]. It provides improvements in memory and language [12] and may allow patients to remain independent for a longer period: after taking rivastigmine, PD patients 
score significantly higher on autonomy in activities of daily living scales [11]. Nevertheless, the question remains whether these improvements are clinically relevant. A further concern is that, although generally well tolerated, this medication may induce adverse effects, such as increased tremor, nausea or vomiting. Other pharmacological treatments that are prescribed for cognitive decline in PD are donepezil, galantamine, and memantine, and although these seem to have some effect on cognition, they are considered to have only slight benefits. Because of the limited effectiveness of medication in alleviating cognitive symptoms, researchers are looking for alternatives in the form of non-pharmacological treatments.

Cognitive training involves structured and theoretically driven practice of tasks that target cognitive domains. Depending on the type of training, cognitive training can improve cognitive functions or enhance compensation strategies. Analogous to the way a person's physical fitness is improved by exercising the body, a person's cognitive fitness could be maintained or improved by exercising the mind.

The aging brain is thought to retain some degree of plasticity [13], suggesting that cognitive training programs may may benefit adults. Many studies on cognitive training have been performed in healthy older adults or adults with cognitive impairment, and the results showed that these trainings were able to improve cognitive abilities [14-16]. In the last couple of years, a small number of cognitive intervention-studies have been conducted in PD patients. Some have shown that cognitive training interventions improved memory performance [17] and overall cognition [18] after six weeks of training. In other studies, positive effects were also found on processing speed and executive function. More importantly, six weeks of cognitive training reduced the risk of developing PD-MCl after a one-year follow up assessment (one year after training) [18]. In a systematic review, Leung et al. [19] demonstrated a modest effect of cognitive training on cognitive function in patients with mild to moderate PD. Conclusively, Leung et al. [19] suggested that larger sample sizes with longer follow-ups were needed to examine the effectiveness of cognitive trainings in preventing cognitive decline in PD. More recently, a systematic review found positive effects of computer-based multimodal cognitive training on most cognitive domains [20]. Since the functional network activity changes in PD are yet unclear, future imaging studies could provide a better understanding of those changes in PD patients as a result of cognitive training [21].

\section{GAMIFICATION AND VIDEO GAMES}

Traditional cognitive trainings involve repetitive execution of cognitive tasks. This is generally considered an effective method to train cognition, but it is also a mundane exercise, which possibly hampers long-term adherence. Converting monotonous tasks into attractive, playful exercises may increase adherence and thus the effectiveness of such therapies. Introducing playful elements in trainings is also known as gamification: gaming principles, such as reward and engagement, are added to a non-game (serious) context. Through gamification, personalization of the training content can be established, and this could increase the adherence to and effectiveness of cognitive training in PD. Although some cognitive training 
programs have been adapted to the user performance, adherence rates were unfortunately insufficiently reported across studies. More research is currently required, since we are unable to conclude whether gamification of cognitive training is indeed more attractive for PD patients and increases adherence to such interventions.

\section{DIGITAL TECHNOLOGY-ENABLED HOME HEALTH CARE}

Web-based and digital technology-enabled therapies introduce a radical shift in health care delivery: instead of treating patients in the hospitals, caregivers are now able to deliver (preventative) care in the home situation. Especially in the midst of the COVID-19 pandemic, digital care technologies have been catalysed as impactful solutions to continue delivering high quality care in the home without physical interactions between caregivers and patients, thereby preventing exposure to potentially infectious people [22]. Digital technology-enabled home health care has other benefits as well: patients can now better monitor and manage their own health and wellbeing more frequenctly and, if the clinical impact on diseases is sufficiently large, home-based therapies can extend independent living as well as serve as prevention measures for both hospital admissions and readmissions [23]. Therapies such as cognitive training could be specifically suited for a home-based alternative, and although not all types of care are equally suited for digital alternatives, even medical treatments can benefit from digital technologies such as digital medicine support. Moreover, effective digital home care outside of the hospital could result in major cost-reductions in health care systems [24]. Such technological home care solutions should be well designed and tested, and it should be investigated thoroughly whether such solutions have beneficial effects on cost-reduction in the long run as well [25].

\section{MONITORING TREATMENT PROGRESSION}

There is an extensive choice of standard cognitive tests available that have proven to be valid, reliable, and sensitive in measuring specific aspects of cognitive functioning. These assessments are currently essential in establishing $\mathrm{MCl}$ or dementia in a patient, but they are time-consuming, because they require involvement of a professional to execute the assessment. For treatment evaluation or for monitoring progression, however, a timeconsuming assessment is undesirable and perhaps redundant; ideally, a brief and, if possible, home-based measurement will already provide meaningful insights. To achieve this, development of new and faster cognitive assessment alternatives is needed. A web-based cognitive assessment has potential benefits over traditional cognitive assessments, since they can be standardized and do not require specialist supervision, thus preventing timeconsuming and expensive assessments that are restricted to a clinical setting. Web-based delivery of cognitive tests opens up the possibility of home-based assessments, which on the one hand might be both less burdensome for patients and more cost- and time-efficient, and, on the other hand, provide a more real-time cognitive measuring rod, monitoring the progression of therapy over time at multiple time points. 


\section{ONE STEP AT A TIME}

Creating an effective home-based gamified cognitive training that delays (the onset of) cognitive decline is the ultimate goal of research in this area. Yet, prior to taking that leap, there are a few smaller steps that need to be taken in support of such new cognitive trainings. It would be helpful to explore whether PD patients are interested in using gamified cognitive trainings at all and ascertain what elements these game interventions would have to include. First, this can give insight into the motivations of PD patients to perform such an intervention. Second, it would be interesting to see what elements future (gamified) cognitive training programs should contain to increase adherence and thereby the efficacy of such programs. Third, in order to be able to evaluate and monitor treatment progression remotely, a web-based cognitive outcome measure in the form of online cognitive assessments should be designed and evaluated.

\section{AIMS AND OUTLINE OF THE PRESENT THESIS}

This thesis outlines several studies and publications on gamification, cognitive training, and Parkinson's disease. My aim was to analyze the prerequisites of gamification in homebased cognitive training in PD, and to investigate the adherence to and efficacy of such interventions in PD. We designed this project to contain a couple of umbrella phases. In Phase A, we developed a gamified cognitive training in collaboration with patients and a cognitive gamification company (MyCognition Ltd.), aiming to create a science-based training program that is both attractive to play and effective. In Phase B-1, we conducted a pilot study on the feasibility of such a gamified cognitive training in $\mathrm{PD}-\mathrm{MCl}$, where the adherence to a 24-week training program was evaluated. In Phase B-2, we conducted a pilot study on the preliminary effects of such a gamified cognitive training. An active intervention group was compared to a waiting list control group after 12 weeks of home-based gamified cognitive training (aimed at exercising the cognitive domains of working memory, episodic memory, attention, psychomotor speed and executive functioning).

These phases can be considered as preparatory work and a starting position for a later Phase $\mathrm{C}$. This future phase involves the conduction of a large study on the efficacy of an improved gamified home-based cognitive training in PD-MCl, in which all lessons learned from the previous phases will be incorporated into an optimized training program and refined study design.

In Chapter 2, I will outline the problem of cognitive decline in PD and investigate the current knowledge on the biological basis for cognitive training in PD. The underlying pathophysiology of cognitive impairment is still unclear. Several alterations in functional network connectivity have been described in persons with PD experiencing cognitive impairment, but the reported resting-state connectivity patterns vary greatly, perhaps due to the heterogenous pathophysiology underlying cognitive decline. By pooling data from available studies, we evaluated the localization and magnitude of functional connectivity patterns in resting-state networks in PD patients with cognitive impairment. We aimed to gain a better understanding of the pathophysiology of cognitive impairment and we aimed to find a possible biomarker, which relates to the creation of better outcome measures. 
The study in Chapter 3 aimed to outline the current knowledge as well as future directions for cognitive training in PD. Because of the limited effectiveness of pharmacological treatments, non-pharmacological interventions are increasingly acknowledged as alternatives to treat or reduce functional limitations in PD. Aerobic exercise is an example of a widely used therapy in this regard, improving motor function but also mood and cognitive function [26]. Computerbased cognitive training is a relatively new intervention that brings various novel elements to the table. We explored the current knowledge on computer-based cognitive training and discussed the usability of its innovative elements.

Chapter 4 reports on the attractiveness of and motivation for gamified cognitive training in the PD population. As it is important to gauge the interest in gamified cognitive training prior to conducting large trials, this chapter shows a promising interest in gamified cognitive training in Dutch PD patients. This interest is shown by presenting three separate cases of self-invented gamified cognitive training, as well as by presenting the recruitment results of the Parkin'Play study.

Chapter 5 evaluates the validation of the $\mathrm{MyCQ}^{\mathrm{TM}}$ cognitive quotient: a brief online cognitive assessment, which is used in the Parkin'Play study to tailor the training program to the individual cognitive performance levels of a subject. In cognitive intervention studies, assessment of cognitive performance is imperative, but the heterogeneity of cognitive assessment batteries impedes the comparability between studies. Moreover, reliable cognitive assessment batteries are time-consuming, are usually confined to a clinical setting, and are therefore expensive. We aimed to validate a brief online cognitive assessment in a mixed psychiatric population for future in gamified home-based cognitive trainings.

In Chapter 6, the design of the Parkin'Play study (pilot) is presented. The Parkin'Play Study is the first large-scale attempt to investigate the feasibility and efficacy of a gamified homebased cognitive training in a PD population. PD patients with mild cognitive impairment were randomly allocated to a passive control group of active intervention group, which received a gamified cognitive intervention at home for up to 24 weeks. Cognition was assessed at twelveweek intervals. Secondary outcome measures include neuropsychological questionnaires and adherence results.

Chapter 7 presents the interim results of our Parkin'Play study in a group of 41 subjects, with a strong focus on adherence. Although gamification may enhance traditional cognitive training with regard to attractiveness and adherence, the merits of gamified cognitive training in PD have never been investigated. Previous studies on (gamified) cognitive training did not report feasibility outcomes. We report the feasibility results of the Parkin'Play study pilot and discuss potential procedural improvements that should be considered to enhance the design of future larger trial. Additionally, (preliminary) efficacy results of the pilot study are presented.

The last chapter gives a summary of the results presented in this thesis. In the general discussion, implications for future research are discussed. 


\section{REFERENCES}

1. Dorsey ER, Sherer T, Okun MS, Bloem BR. The Emerging Evidence of the Parkinson Pandemic. Brundin P, Langston JW, Bloem BR, editors. Journal of Parkinson's disease IOS Press; 2018;8(s1):S3-S8. PMID: 30584159

2. Goldman JG, Litvan I. Mild cognitive impairment in Parkinson's disease. Minerva medica NIH Public Access; 2011;102(6):441.

3. Yarnall AJ, Breen DP, Duncan GW, Khoo TK, Coleman SY, Firbank MJ, et al. Characterizing mild cognitive impairment in incident Parkinson disease The ICICLE-PD Study. Neurology AAN Enterprises; 2014;82(4):308-316.

4. Hely MA, Reid WGJ, Adena MA, Halliday GM, Morris JGL. The Sydney multicenter study of Parkinson's disease: The inevitability of dementia at 20 years. Mov. Disord. Wiley-Blackwell; 2008 Feb 28;23(6):837-844.

5. Pigott K, Rick J, Xie SX, Hurtig H, Chen-Plotkin A, Duda JE, et al. Longitudinal study of normal cognition in Parkinson disease. Neurology Lippincott Williams \& Wilkins; 2015 Oct 13;85(15):1276-1282. PMID: 26362285

6. Petersen RC, Lopez O, Armstrong MJ, Getchius TSD, Ganguli M, Gloss D, et al. Practice guideline update summary: Mild cognitive impairment: Report of the Guideline Development, Dissemination, and Implementation Subcommittee of the American Academy of Neurology. Neurology. Wolters Kluwer Health, Inc. on behalf of the American Academy of Neurology; 2018. p. 126-135. PMID: 29282327

7. Bellucci A, Mercuri NB, Venneri A, Faustini G, Longhena F, Pizzi M, et al. Review: Parkinson's disease: from synaptic loss to connectome dysfunction. Neuropathol Appl Neurobiol 2016 Feb;42(1):77-94. PMID: 26613567

8. Chaudhuri KR, Schapira AHV. Non-motor symptoms of Parkinson's disease: dopaminergic pathophysiology and treatment. The Lancet Neurology 2009 May;8(5):464-474. PMID: 19375664

9. Garcia-Diaz Al, Segura B, Baggio HC, Marti MJ, Valldeoriola F, Compta Y, et al. Parkinsonism and Related Disorders. Parkinsonism and Related Disorders Elsevier Ltd; 2014 Dec 1;20(12):1405-1410. PMID: 25457818

10. Nagano-Saito A, Habak C, Mejía-Constaín B, Degroot C, Monetta L, Jubault T, et al. Neurobiology of Aging. Neurobiology of Aging Elsevier Ltd; 2014 Jan 1;35(1):223-231.

11. Olin JT, Aarsland D, Meng X. Rivastigmine in the treatment of dementia associated with Parkinson's disease: effects on activities of daily living. Dementia and geriatric cognitive disorders 2010;29(6):510-515. PMID: 20523050

12. Schmitt FA, Aarsland D, Bronnick KS, Meng X, Tekin S, Olin JT. Evaluating rivastigmine in mild-to-moderate Parkinson's disease dementia using ADAS-cog items. American journal of Alzheimer's disease and other dementias SAGE PublicationsSage CA: Los Angeles, CA; 2010 Aug;25(5):407413. PMID: 20392860 
13. Jones S, Nyberg L, Sandblom J, Stigsdotter-Neely A, Ingvar M, Magnus Petersson K, et al. Cognitive and neural plasticity in aging: general and task-specific limitations. Neurosci Biobehav Rev 2006;30(6):864-871. PMID: 16904746

14. Ngandu T, Lehtisalo J, Solomon A, Levälahti E, Ahtiluoto S, Antikainen R, et al. A 2 year multidomain intervention of diet, exercise, cognitive training, and vascular risk monitoring versus control to prevent cognitive decline in at-risk elderly people (FINGER): a randomised controlled trial. The Lancet 2015 Mar;385(9984):2255-2263.

15. Anguera JA, Boccanfuso J, Rintoul JL, Al-Hashimi O, Faraji F, Janowich J, et al. Video game training enhances cognitive control in older adults. Nature 2013 Sep 4;501(7465):97-101.

16. Nouchi R, Taki Y, Takeuchi H, Hashizume H, Akitsuki Y, Shigemune Y, et al. Brain Training Game Improves Executive Functions and Processing Speed in the Elderly: A Randomized Controlled Trial. Aleman A, editor. PLoS ONE 2012 Jan 11;7(1):e29676.

17. Naismith SL, Mowszowski L, Diamond K, Lewis SJG. Improving memory in Parkinson's disease: A healthy brain ageing cognitive training program. Mov. Disord. 2013 Apr 29;28(8):1097-1103.

18. Petrelli A, Kaesberg S, Barbe MT, Timmermann L, Rosen JB, Fink GR, et al. Cognitive training in Parkinson's disease reduces cognitive decline in the long term. Eur J Neurol 2014 Dec 22;:n/a-n/a. PMID: 25534579

19. Leung IHK, Walton CC, Hallock H, Lewis SJG, Valenzuela M, LampitA. Cognitive training in Parkinson disease: A systematic review and meta-analysis. Neurology 2015 Oct30;85(21):1843-1851. PMID: 26519540

20. Nousia A, Martzoukou M, Tsouris Z, Siokas V, Aloizou A-M, Liampas I, et al. The Beneficial Effects of Computer-Based Cognitive Training in Parkinson's Disease: A Systematic Review. Arch Clin Neuropsychol 2020 Jan 14;13(4):217. PMID: 31942917

21. Alzahrani H, Venneri A. Cognitive Rehabilitation in Parkinson's Disease: A Systematic Review. Journal of Parkinson's disease IOS Press; 2018;8(2):233-245. PMID: 29614698

22. Peek N, Sujan M, Scott P. Digital health and care in pandemic times: impact of COVID-19. BMJ Health Care Inform 2020 Jun 21;27(1):e100166-3.

23. Roy S, Jha R, Karnes A, Oates P, Wojciechowski K, Dvorak A, et al. Innovative eHealth at Home Program Reduces Hospitalization and Readmission Rates of Patients with Heart Failure. Journal of Cardiac Failure Elsevier B.V; 2019 Aug 1;25(Supplement):S127.

24. Jiang X, Ming W-K, You JH. The Cost-Effectiveness of Digital Health Interventions on the Management of Cardiovascular Diseases: Systematic Review. J. Med. Internet Res. 2019;21(6):e13166-11.

25. Rahimi K. Comment Digital health and the elusive quest for cost savings. The Lancet Digital Health The Author(s). Published by Elsevier Ltd. This is an Open Access article under the CC BY 4.0 license; 2019 Jun 19;1(3):e108-e109.

26. Smith PJ, Blumenthal JA, Hoffman BM, Cooper H, Strauman TA, Welsh-Bohmer K, et al. Aerobic exercise and neurocognitive performance: a meta-analytic review of randomized controlled trials. Psychosom Med 2010 Apr;72(3):239-252. PMID: 20223924 


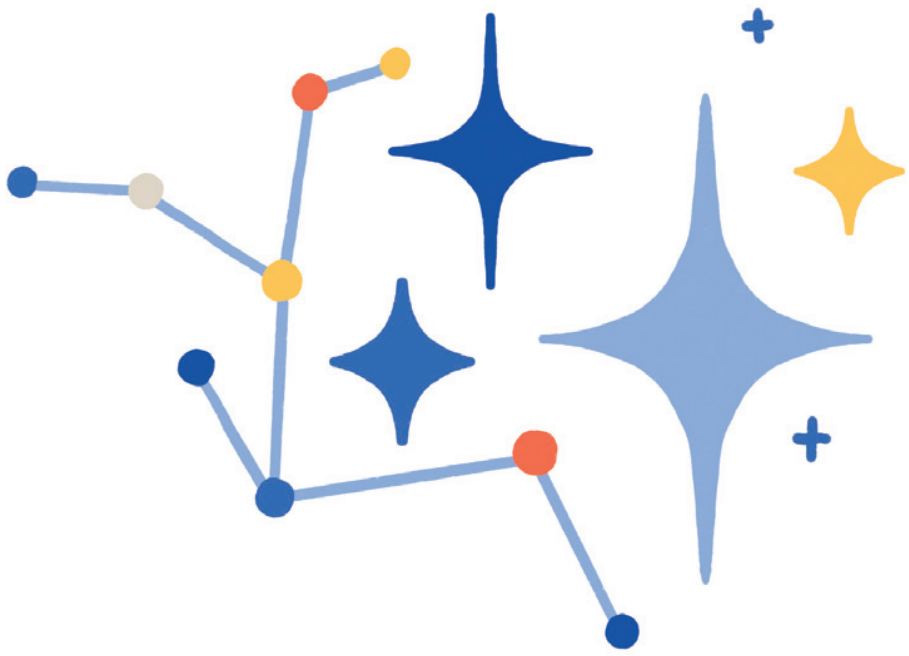




\section{CHAPTER 2}

\section{Resting-state fMRI in}

Parkinson's disease patients

with cognitive impairment:

A meta-analysis 


\section{ABSTRACT}

Background: Cognitive impairment is a common non-motor symptom in Parkinson's disease. So far, the underlying pathophysiology remains unclear. Several alterations in functional network connectivity have been described in Parkinson's disease patients with cognitive impairment which are probably the result of the heterogenous pathophysiology underlying this cognitive decline, including dopaminergic and cholinergic deficits. Accordingly, the reported resting-state connectivity patterns vary greatly among studies.

Objective: To evaluate the localization and magnitude of functional connectivity patterns in resting-state brain networks in Parkinson's disease patients with cognitive impairment by pooling data from available studies.

Methods: We searched PubMed, the Cochrane Library, MEDLINE, Embase and PsycINFO to identify functional MRI studies in Parkinson's disease patients with cognitive impairment. A voxel-based meta-analysis combined with quality statistics was performed, using the anisotropic effect-size version of the signed differential mapping method.

Results: Eighteen studies with cognitively impaired Parkinson's disease patients were included consisting of 236 Parkinson's disease patients with mild cognitive impairment, 68 patients with Parkinson's disease dementia, 320 cognitively unimpaired Parkinson's disease patients and 353 healthy controls. Parkinson's disease patients with cognitive impairment predominantly showed a reduced connectivity in specific brain regions that are part of the default mode network.

Conclusion: Cognitive impairment in Parkinson's disease is associated with reduced connectivity in networks relevant to cognition, most prominently the default mode network. Specific alterations in functional connectivity may contribute to cognitive decline in Parkinson patients and may be a promising future biomarker. 


\section{INTRODUCTION}

Parkinson's disease (PD) is the second most common neurodegenerative disorder after Alzheimer's disease and is characterized by motor symptoms such as bradykinesia, rigidity and tremor [1-3]. Moreover, patients with PD also experience a broad spectrum of non-motor symptoms such as neuropsychiatric disturbances and autonomic dysfunction. Already in the earliest phases of the disease, cognitive impairment $(\mathrm{Cl})$ can be found in up to $42.5 \%$ of the PD patients [4]. Furthermore, up to $80 \%$ of the patients eventually develop Parkinson's Disease Dementia (PDD) in the advanced stages of the disease [5, 6]. Patients with PD experiencing mild cognitive impairment $(\mathrm{MCl})$ are at a higher risk of subsequently developing PDD [7].

The pathophysiological mechanism of cognitive impairment in Parkinson's disease patients has not yet been elucidated and no valid biomarkers have been identified [8]. Research in this field has focused on the formation of protein aggregates, neurotransmitter system dysfunction as well as genetic risk factors and underlying pathways. Lewy-body depositions, formed by neuronal alpha-synuclein aggregates, cause the loss of mesencephalic dopaminergic neurons and result in the typical dopaminergic deficit in the basal ganglia of PD with $\mathrm{Cl}$ [9-12]. In addition, differences in cerebral levels of $A \beta$-amyloid depositions as shown in PET-studies suggest a differential role in cognitive deterioration and development of dementia in PD patients [13]. A previous study of Compta et al. (2011) suggested that it is the combination of cortical amyloid deposits and cortical Lewy bodies that has the most predictive value for the development of $\mathrm{Cl}$ in PD [14]. The spatial distribution of neuronal dysfunction also plays a role in the "dual syndrome hypothesis" [15]. This suggests that (1) dopaminergic dysfunction in the fronto-striatal regions is more involved in a subgroup of PD patients with $\mathrm{MCl}$ and predominantly deficits in planning, working memory and executive functions, whereas (2) cholinergic dysfunction within the posterior cortical and temporal lobes is more involved in early deficits in visuo-spatial function and semantic fluency and a more rapid cognitive decline to dementia [15]. Indeed, several lines of research indicate that besides dopaminergic dysfunction the noradrenergic, serotonergic and cholinergic systems are also affected in PD patients. Dysfunctional neurotransmitter synaptic activity within the locus coeruleus [16-18], dorsal raphe nuclei [19, 20], and cholinergic brainstem nuclei [21] respectively have all been associated with degeneration within these structures in PD. However, as suggested by the dopaminergic overdosing theory, dysfunction of the relatively less effected ventral cortico-striatal circuits, involved in reward processing and learning, may also arise due to an overdose of dopamine in these areas. [15, 22-25]. From another perspective, several genotypes, such as APOE $\varepsilon 4$, MAPT, $\mathrm{H} 1$ haplotypes and GBA mutation [26-30] are increasingly recognized as potential risk factors for dementia in PD and may shed new light in pathophysiological mechanisms of PD-MCI [31-34]. This also points to the direction that there may be several mechanisms involved in $\mathrm{Cl}$ and the development of dementia in PD [8]. 
Of interest, several neuroimaging techniques are able to characterize the pathological substrates of PD and other neurodegenerative disorders [35]. One promising method is resting-state functional MRI, which has shown the ability to explore the functional activity in different brain networks in a reliable and reproducible way [36, 37]. It has been reported that alterations in several neurotransmitter systems influence the functional brain activity measured with fMRI [38-42]. Additionally, a correlation was reported between the loss of dopaminergic neurons and alterations in functional brain network activity in PD [43]. Since the basal ganglia are part of neuronal networks involving the entire cortex, one can expect that reductions in dopaminergic release will affect the functioning of many large-scale cerebral networks relevant for cognitive processing [44]. Furthermore, it has been suggested that an abnormal alpha-synuclein level in the cerebrospinal fluid influences both sensorimotor and non-motor functional connectivity networks in PD [45]. Based on these results, there has been growing interest in using functional magnetic resonance imaging (fMRI) to investigate the neural basis for $\mathrm{Cl}$ in PD. By measuring intrinsic blood oxygen level-dependent (BOLD) low-frequency signal fluctuations, fMRI can be used to detect interregional correlations in specific brain networks during rest [46, 47].

Various studies have investigated the resting-state networks in PD-MCl and PDD and disruptions are predominantly described in the default mode network and the fronto-parietal network [37, 48-54]. The default mode network is thought to serve an important role in several higher order cognitive functions, such as autobiographical memory and imagining the future, while the fronto-parietal network is predominantly involved in attention and cognitive control [37, 55]. Unfortunately, results between studies vary greatly and most fMRI studies consist of small samples.

In this study, we pool functional connectivity data in resting-state brain networks of PD patients with $\mathrm{Cl}$ by means of a voxel-based meta-analysis. The aim of this study is to obtain a better understanding of the functional connectivity networks involved in PD with $\mathrm{Cl}$ and to provide evidence as to whether $\mathrm{FMRI}$ results could serve as a biomarker for PD with $\mathrm{Cl}$. Based on previous studies, we hypothesize that PD patients with $\mathrm{Cl}$ show reduced resting-state brain connectivity compared to healthy controls and PD patients without $\mathrm{Cl}$. We specifically expect to observe these alterations most prominently in the default mode network and fronto-parietal network.

\section{METHODS}

\section{SEARCH STRATEGIES AND STUDY SELECTION}

A literature search in PubMed, Medline, Embase, PsycINFO and the Cochrane Library was performed. For this search, the following search terms were used: ((Parkinson's disease) OR (Parkinson)) AND ((Mild cognitive impairment) OR (MCI) OR (Parkinson's disease dementia) OR (Dementia) OR (Cognitive impairment)) AND ((fMRI) OR (functional MRI)). Afterwards, the reference lists of the included articles were searched for additional eligible publications. The final search was conducted on the 30th of April 2018 and resulted in a total of 1122 articles. 
For this meta-analysis, we included resting-state fMRI studies comparing a group of cognitively impaired PD patients (PD-CI) with either a sample of healthy controls $(\mathrm{HC})$ or a sample of PD patients without $\mathrm{Cl}$. Studies comparing the functional connectivity patterns of $\mathrm{HC}$ or PD with dementia with Lewy bodies were not included. Furthermore, only studies applying a wholebrain analysis, independent component analysis or seed-based analysis, with a correlation of the seeds to the entire brain, were included in this meta-analysis. To prevent the results from being biased towards certain region of interest, studies performing a region of interest analysis were excluded. Other exclusion criteria were: 1 . Undefined PD with $\mathrm{Cl}$ study groups or not enough information provided to determine whether $\mathrm{Cl}$ was present; 2 . No resting-state fMRI; 3. Review articles reporting no original data; or 4. Conference proceedings without full report publication. Three manuscripts had to be excluded because they focused on global connectivity and network topological parameters and did not report specific changes on a regional or voxel-level [56-58].

First, duplicates were removed from the search results, followed by screening of the abstracts independently by two researchers (AW, SW). When any discrepancies existed between the included articles, this was resolved in a consensus meeting. When no consensus was reached, a third specialist was consulted (MK). The following information was extracted from each included study: first author, year of publication, published journal, sample size, MRI type, definition of $\mathrm{Cl}$, statistical analysis technique and patient characteristics (UPDRS score, age, dopaminergic medication, LEDD, and MMSE or MoCA-score). Peak coordinates and effect size measures of the regions with a significant difference in functional connectivity were also collected. Some publications did not report peak coordinates and therefore the relevant authors were contacted by e-mail to request this information.

\section{QUALITY ASSESSMENT}

To our knowledge, there are no official guidelines for assessing the quality of fMRI studies. Therefore for the overall quality assessment of the reports in this meta-analysis, we derived our own criteria from the guidelines for reporting fMRI studies as described by Poldrack et al. (2009) [59]. This resulted in nine quality criteria, which comprise of the following domains: (1) Inclusion and exclusion procedure and patient demographics; (2) fMRI procedure and patient instructions; (3) Spatial normalization method; (4) Determination of the regions of interest; (5) Reproducibility of the analysis; (6) Statistical tests used to substantiate the results; (7) Correction for the multiple testing problem; (8) Figures and tables; (9) Quality control measures. Studies could score $0,0.5$ or 1 point for each item. An overall score of $\geq 7.5$ was considered as good, 4-7.5 as fair and $\leq 4$ as poor quality. See supplementary data S1 for further specification of the criteria. Quality assessment was performed by two researchers (AW, SW) and discrepancies were discussed until consensus was reached. If no consensus could be reached, a third specialist was consulted $(\mathrm{HJ})$. 


\section{DATA ANALYSIS}

This meta-analysis was carried out using the anisotropic effect size version of signed differential mapping (AES-SDM) [60, 61]. This validated voxel-based meta-analyses approach has been used in meta-analysis of several other neuropsychiatric studies [62-67]. AES-SDM is specifically designed to combine neuroimaging studies with studies reporting solely peak coordinates in coordinate systems (e.g. MNI, Talairach). The peak coordinates and their statistical values are used to recreate a statistical parametric map for each study. This map is created by using the effect sizes of the differences between patients and controls. Subsequently, a randomeffects variance-weighted image-based meta-analysis is conducted in each voxel. In this meta-analysis, we applied the default AES-SDM kernel size and thresholds (FWHM $=20 \mathrm{~mm}$, voxel $p=0.005$, peak height SDM-Z $=1$, cluster extent $=10$ voxels). The SDM-Z score represents a probability measure. In the random-effects analysis the results are thresholded to this given probability, to detect if more studies report functional connectivity changes near a certain voxel than would be expected by chance $[61,62,68]$.

To assess the residual heterogeneity of the results, examination of the funnel plots of the peaks coordinates was carried out. This allowed us to check whether the results were driven by one or very few studies and to detect gross differences in study results. These funnel plots were statistically tested with Egger's regression test. To further assess the robustness of the results, we performed a jack-knife analysis within AES-SDM, in which the meta-analysis is systematically repeated as many times as studies have been included, subsequently removing one different study at a time.

After performing the statistical analysis, the spatial layout of these results was compared with the intrinsic connectivity network templates as described by Smith et al. (2009) [69]. These templates were obtained from the BrainMap database [70-72]. We performed a spatial correlation analysis in FSL to determine the degree of spatial overlap between our map with peak coordinate clusters and these well-defined functional networks. This also includes a calculation of the number of voxels of our peak coordinate clusters inside the resting-state network templates as a percentage of the total number of voxels in the clusters. Afterwards we visually inspected these images to identify which peak coordinates led to the corresponding spatial correlation value.

\section{RESULTS}

\section{DEMOGRAPHIC DATA AND QUALITY ASSESSMENT}

Seventeen studies met the in- and exclusion criteria and were included in this meta-analysis $[48,49,52,73-86]$. Among these, fifteen studies compared PD-Cl with healthy controls (HC) and nine studies compared PD-Cl patients to PD patients who were cognitively unimpaired (PDCU). Two studies, from Madhyastha et al. (2015) and Canu et al. (2015), reported results for the comparison of $\mathrm{HC}$ to a group of $\mathrm{PD}$ patients comprising of both $\mathrm{CU}$ patients and patients with 
$\mathrm{MCI}[48,76]$. Both studies were included because, firstly, the sample size of PD contained more $\mathrm{MCI}$ than PD-CU patients and, secondly, the PD group as a whole scored considerably worse on the neuropsychological assessment compared to the control group. The complete in- and exclusion procedure is displayed in figure 1. Altogether, these studies included 932 participants of which $353 \mathrm{HC}, 289$ PD-CU, $222 \mathrm{PD}-\mathrm{MCl}$ and 68 patients with PDD. Basic demographics of the participants per group are summarized in table 1 . Baseline characteristics showed a higher mean age for PDD (71.5) and PD MCI (66.9) when compared to PD CU (63.6) and HC (64.5). Additionally, as expected the PD MCI and PDD groups showed worse results regarding the global cognition scores. Moreover, higher mean LEDD and UPDRS-III scores were found in the $\mathrm{PD}-\mathrm{MCl}$ and PDD subgroup, indicating more advanced stages of the disease.

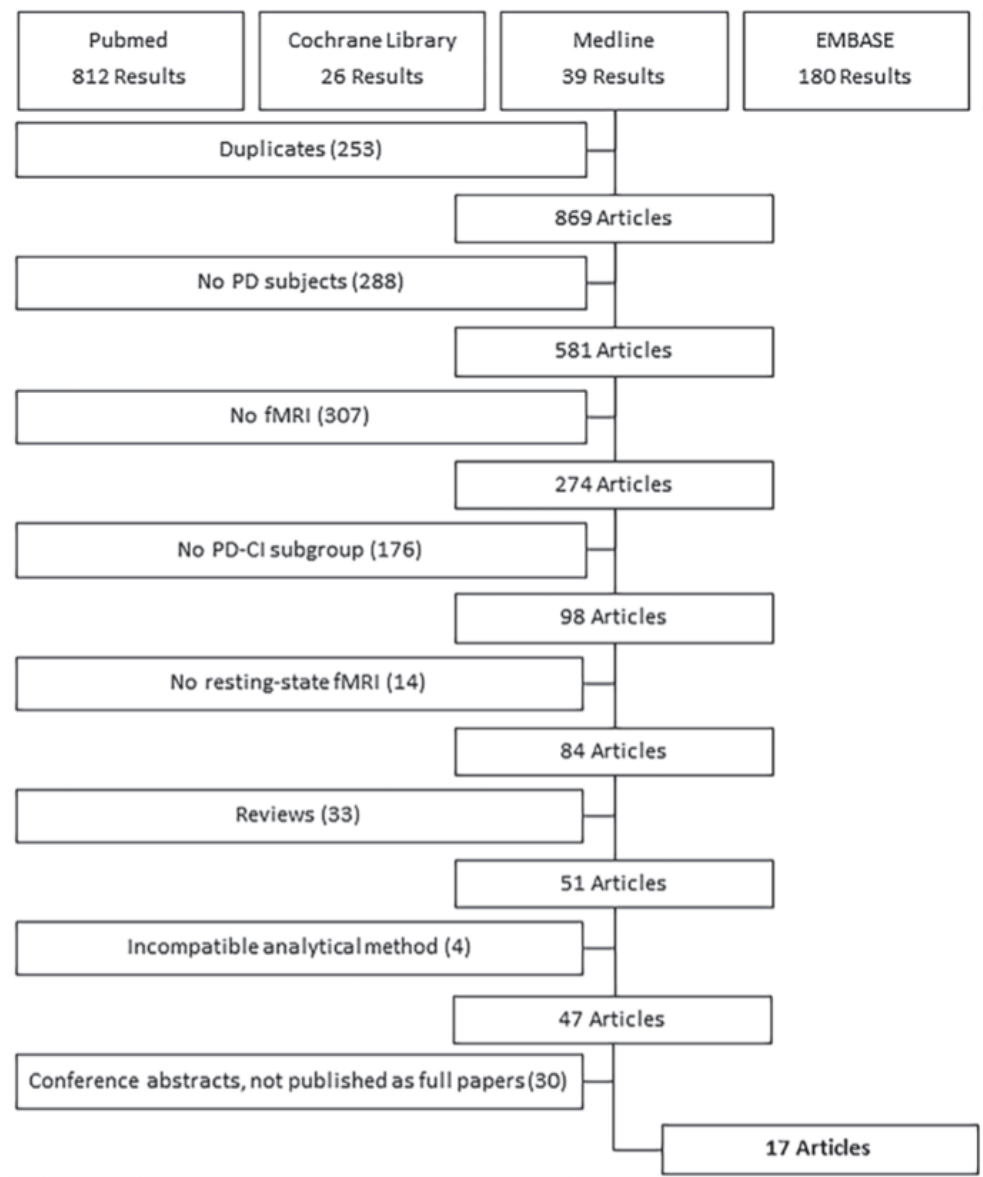

Figure 1. Flowchart of the study selection procedure, performed according to the PRISMA 2009 guidelines.

The fMRI characteristics and statistical details of the included studies are described in table 2 . Eight of the studies performed a seed-based analysis [49, 52, 78-80, 83, 85, 86], six studies performed an independent component analysis $[48,73,75,76,81,84]$ and the three remaining 
studies adopted different whole brain methodologies [74, 77, 82]. Atrophy correction was applied in only six studies $[52,75,77,79,80,83]$. Twelve studies did not enter the subjects gray matter volume maps as a voxel-wise regressor in the group comparison $[48,49,73,74,76,78,81,82$, 84-86]. However, three of these twelve studies conducted a voxel-based morphometry analysis $[73,76,86]$, which did not show a significant difference between HC and PD-Cl. Another study of Bezdicek et al. (2018) has also performed a voxel-based morphometry which did not show significant differences between PD-MCI and PD-CU, but the analysis did express significant differences in brain atrophy between $\mathrm{HC}$ and PD-MCI for which the results were not corrected [82].

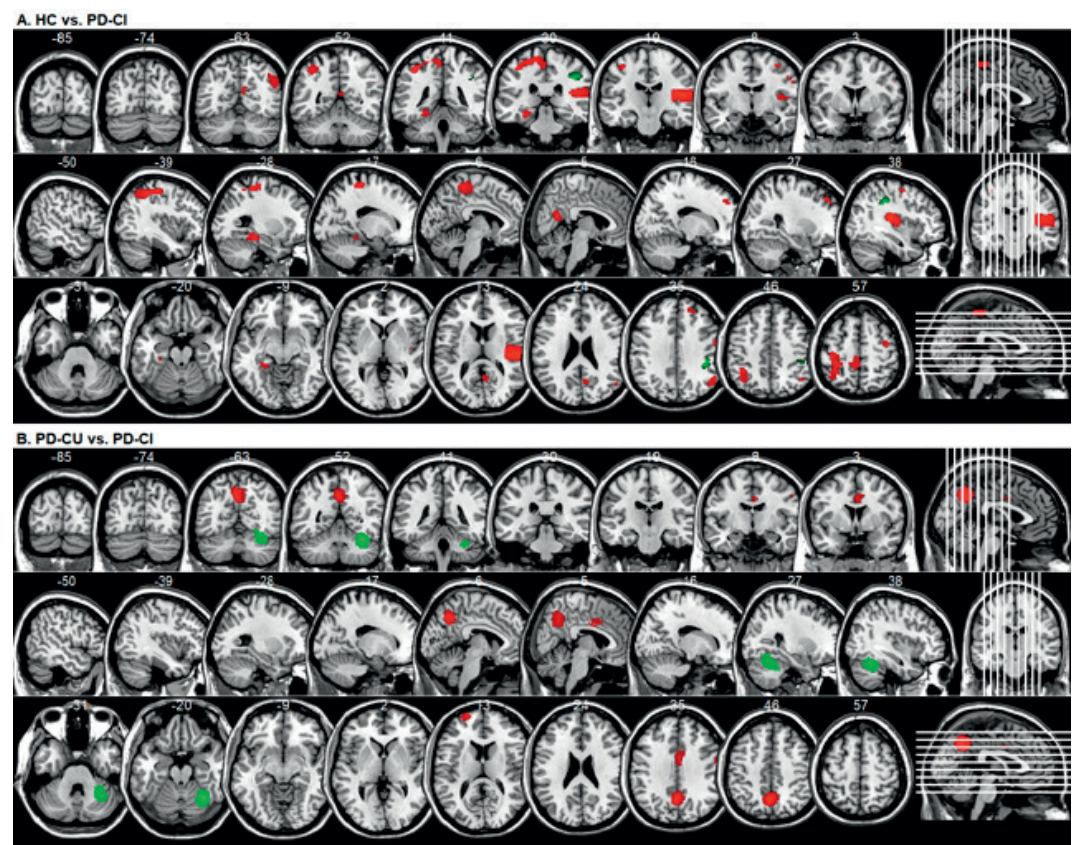

Figure 2. Statistically significant effects of voxel-based meta-analysis for HC vs. PD-CI $[A]$ and PD-CU vs. $\mathrm{PD}-\mathrm{Cl}[\mathrm{B}]$. Decreased functional connectivity in $\mathrm{PD}-\mathrm{Cl}$ is indicated in red and increased connectivity in $\mathrm{PD}-\mathrm{Cl}$ is indicated in green for all contrasts. Voxel threshold $\mathrm{p}<0.005$, peak height threshold: peak SDM-Z $>1.000$, extent threshold: cluster size $\geq 10$ voxels. $x, y, z$-coordinates of axial, sagittal and coronal slices are indicated in white. 
Based on our quality assessment, all included studies reached a score of either 'Good' or 'Fair'. Three studies were classified as fair $[49,83,85]$. The main reasons for this were the lack of motion or atrophy correction, inadequate normalization methods, or the fact that the in- and exclusion criteria and patient instructions for the resting-state fMRI were not clearly reported. All other studies had a total score above 7 and were therefore considered as being of good quality. Further specification of the quality assessment can be found in the supplementary data S1.

\section{REGIONAL CHANGES IN RESTING-STATE CONNECTIVITY}

The meta-analysis showed reduced functional brain connectivity in several brain regions in patients with $\mathrm{PD}-\mathrm{Cl}$ as compared to $\mathrm{HC}$. More specifically, our meta-analysis demonstrated a reduced connectivity in PD-Cl in the right Rolandic operculum, left inferior parietal gyri, right angular gyrus, left parahippocampal gyrus, right calcarine fissure, right superior frontal gyrus and right precentral gyrus as compared to the $\mathrm{HC}$ (see table 3, figure $2 \mathrm{~A}$ ). An increased functional connectivity in PD-Cl was found in the right supramarginal gyrus when compared to the $\mathrm{HC}$ group.

For the contrast PD-CU vs. PD-Cl, lower connectivity was detected in PD-Cl patients in the left precuneus, right median cingulate gyrus, left superior frontal gyrus and right precentral gyrus. In addition, increased functional connectivity of the right cerebellum (hemispheric lobule VI) was found in the PD-Cl group as compared to PD-CU (see table 3, figure 2B).

\section{ROBUSTNESS ANALYSIS}

The robustness analysis was performed with the Egger's linear regression method and visual inspection of the jack-knife analysis. See supplementary data S2 for further specification of the funnel plots and this analysis. The Egger's test showed a significant asymmetry regarding the funnel plot of the right Rolandic operculum, with a p-value smaller than 0.1. None of the other reported peak coordinates in this study showed an intercept that significantly differed from zero $(P>0.1)$. Visual inspection of the jack-knife analysis for the contrast of $\mathrm{HC}$ vs. PD-Cl, exhibited a poor reproducibility of the peak coordinate in the right precentral gyrus (BA 6). With respect to the comparison of $\mathrm{PD}-\mathrm{CU}$ with $\mathrm{PD}-\mathrm{Cl}$ patients, the right cerebellar region (BA 37) showed a somewhat lower reproducibility. 


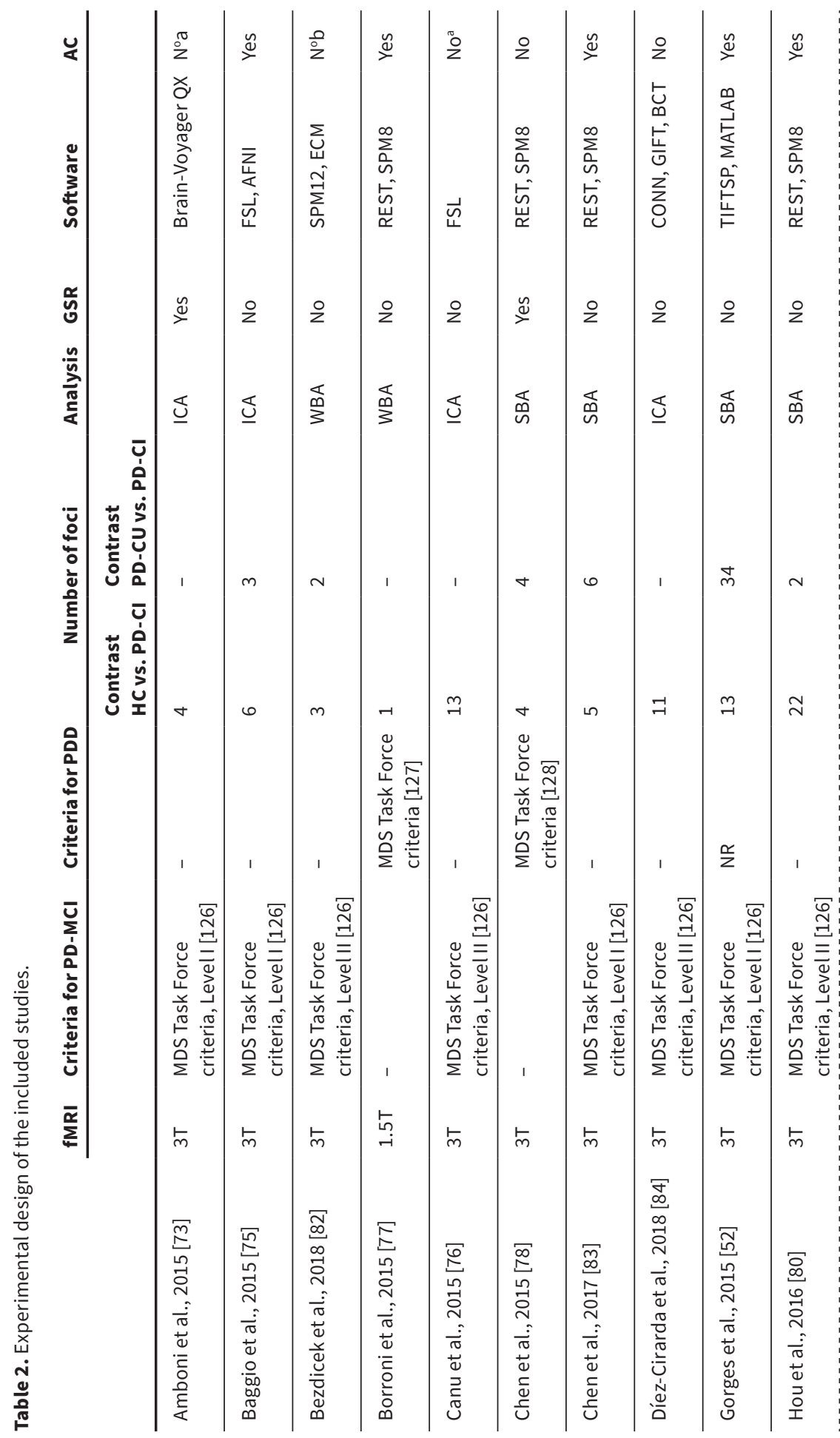


32

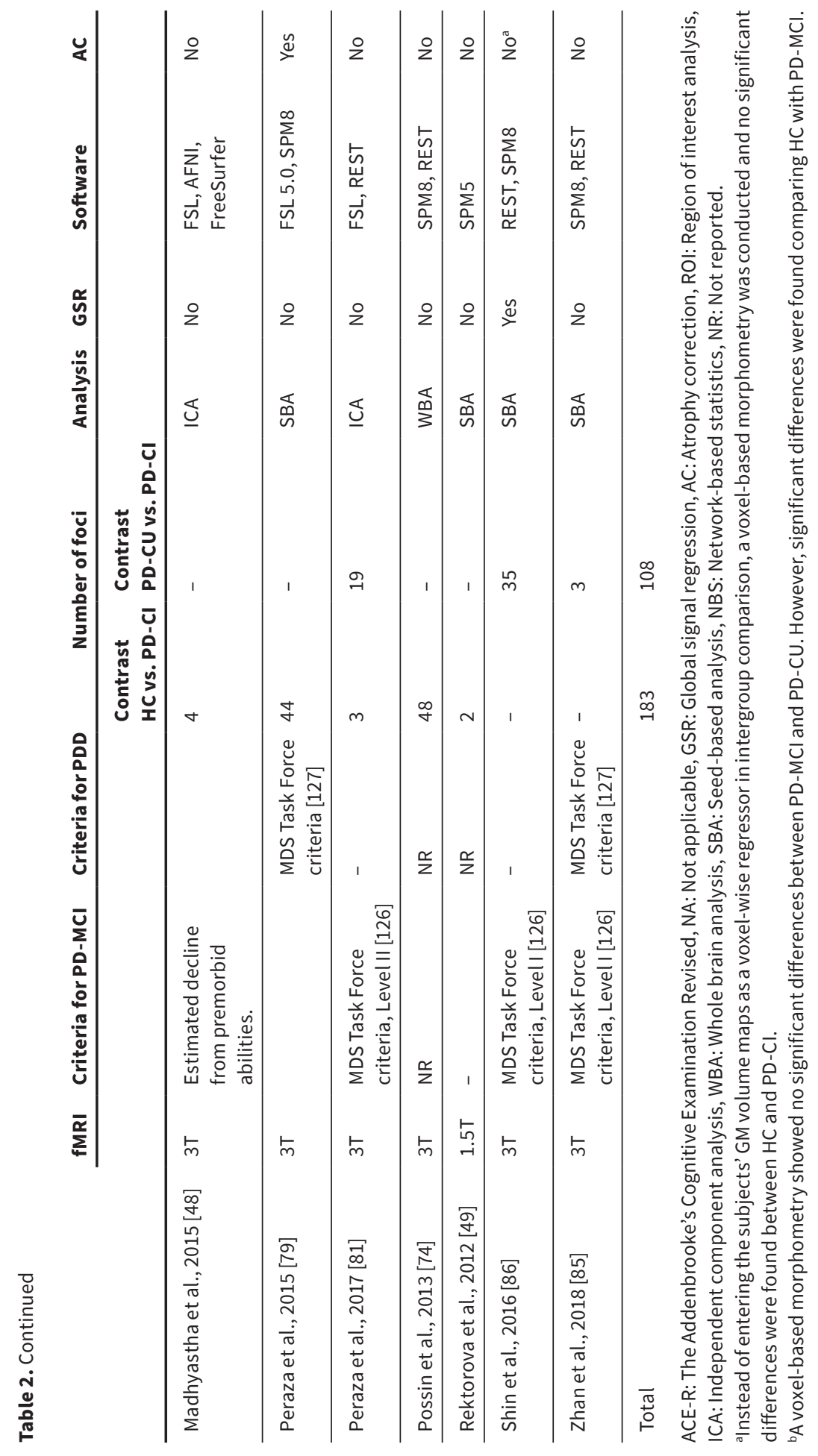


Table 3. Clusters of voxels with significant intergroup functional connectivity differences.

\begin{tabular}{|c|c|c|c|c|c|c|c|c|}
\hline \multirow[t]{2}{*}{ Contrast } & \multirow[t]{2}{*}{ Neural region } & \multirow[t]{2}{*}{ Side } & \multicolumn{3}{|c|}{ MNI coordinates } & Voxels & \multirow[t]{2}{*}{ P-value } & \multirow[t]{2}{*}{ SDM-Z } \\
\hline & & & $\mathbf{x}$ & $\mathbf{Y}$ & $\mathbf{Z}$ & & & \\
\hline \multirow[t]{8}{*}{$\mathrm{HC}>\mathrm{PD}-\mathrm{Cl}$} & Rolandic operculum, BA 48 & Right & 44 & -24 & 16 & 1363 & 0.00002 & -2.705 \\
\hline & Inferior parietal gyri, BA 40 & Left & -38 & -42 & 52 & 1400 & 0.00004 & -2.565 \\
\hline & Angular gyrus, BA 39 & Right & 48 & -58 & 42 & 327 & 0.00089 & -2.159 \\
\hline & Parahippocampal gyrus, BA 37 & Left & -28 & -34 & -14 & 260 & 0.00025 & -2.328 \\
\hline & $\begin{array}{l}\text { Calcarine fissure/surrounding } \\
\text { cortex, BA } 23\end{array}$ & Right & 6 & -60 & 16 & 161 & 0.00056 & -2.221 \\
\hline & $\begin{array}{l}\text { Superior frontal gyrus, } \\
\text { dorsolateral, BA } 9\end{array}$ & Right & 24 & 38 & 40 & 104 & 0.00056 & -2.220 \\
\hline & Precentral gyrus, BA 4 & Right & 54 & -2 & 36 & 67 & 0.00124 & -2.114 \\
\hline & Precentral gyrus, BA 6 & Right & 38 & -8 & 56 & 63 & 0.00143 & -2.093 \\
\hline $\mathrm{PD}-\mathrm{Cl}>\mathrm{HC}$ & Supramarginal gyrus, BA 40 & Right & 38 & -32 & 38 & 277 & $\sim 0$ & 1.243 \\
\hline \multirow[t]{4}{*}{$\mathrm{PDCU}>\mathrm{PD}-\mathrm{Cl}$} & Precuneus, BA 7 & Left & -4 & -60 & 42 & 931 & $\sim 0$ & -2.312 \\
\hline & $\begin{array}{l}\text { Median cingulate/paracingulate } \\
\text { gyrus, BA } 24\end{array}$ & Right & 4 & 2 & 34 & 261 & 0.00051 & -1.820 \\
\hline & $\begin{array}{l}\text { Superior frontal gyrus, } \\
\text { dorsolateral, BA } 10\end{array}$ & Left & -22 & 64 & 14 & 191 & 0.00022 & -1.939 \\
\hline & Precentral gyrus, BA 4 & Right & 56 & -2 & 38 & 53 & 0.00214 & -1.620 \\
\hline $\mathrm{PD}-\mathrm{Cl}>\mathrm{PD} \mathrm{CU}$ & $\begin{array}{l}\text { Cerebellum, hemispheric } \\
\text { lobule VI, BA } 37\end{array}$ & Right & 30 & -58 & -20 & 1083 & 0.00029 & 1.371 \\
\hline
\end{tabular}

Voxel threshold $p<0.005$, peak height threshold: peak SDM-Z $>1.000$, extent threshold: cluster size $\geq 10$ voxels.

\section{NETWORK LOCALIZATION}

We performed a spatial correlation analysis in which we compared the total brain map of our reported peak coordinates with the intrinsic connectivity network templates as described by Smith et al. (2009) to detect in which resting-state networks the peak coordinates are situated. With this analysis, a spatial correlation was found between our reported peak coordinates and several resting-state networks. For all associated networks, we have displayed both the spatial correlation coefficient ( $r$ ) and the percentage of voxels of our peak coordinate clusters inside the resting-state networks as a fraction of the total number of voxels of our peak coordinate clusters. Regarding the contrast of HC vs. PD-Cl, our analysis displayed a correlation between the total map of peak coordinates with a decreased connectivity in PD-Cl and the auditory network $(r=0.13 ; 28,5 \%)$, the sensorimotor network $(r=0.13 ; 30,6 \%)$. Spatial correlation was also found to a lesser extent, in the right fronto-parietal network $(r=0.07 ; 14,4 \%)$ and default mode network ( $r=0.07 ; 17,2 \%)$. Upon visual inspection, the spatial correlation with the auditory network appeared to be driven by the peak coordinate of the right Rolandic 
operculum and right precentral gyrus (BA 4). The observed spatial correlation with the sensorimotor network seemed to be based on the peak coordinate of the left inferior parietal gyri and precentral gyrus (BA 6). Moreover, predominantly the peak coordinate in the right Rolandic operculum explains the spatial correlation with the right fronto-parietal network. And finally, the peak coordinates of the right calcarine fissure and right angular gyrus were mainly associated with the default mode network. The peak coordinate in the right supramarginal gyrus, which showed an increased connectivity in PD-Cl showed a correlation with the sensorimotor network $(r=0.05 ; 90,6 \%)$ and the right fronto-parietal network $(r=0.05 ; 36,1 \%)$.

For our second contrast, PD-Cl vs PD-CU, we observed a decreased connectivity in PD-CI which correlated spatially specifically with the default mode network $(r=0.24 ; 66,2 \%)$. After visual inspection we noticed that the spatial correlation with the default mode network could be attributed to the peak coordinates in the left precuneus, right precentral gyrus and the right median cingulate gyrus. The correlation value of the auditory network was related to the peak coordinates in the left middle temporal gyrus and right precentral gyrus. The peak coordinate in the right cerebellum, which displayed an increased functional connectivity for PD-Cl as compared to PD-CU, did primarily show a notable spatial correlation with the cerebellar network ( $r=0.19 ; 99,7 \%)$. For further specification of the spatial correlation analysis, see supplementary data S3.

\section{DISCUSSION}

In this study, we aimed to evaluate the hypothesis that reduced functional connectivity changes in $\mathrm{PD}$ patients with $\mathrm{Cl}$ can be detected in specific resting-state networks, as the disease induced dopaminergic deficits can have widespread repercussions on brain function. As expected, the found spatial correlation coefficients $(r)$ with specific resting-state networks are rather low. The reason for this is that the results from our meta-analysis contain far fewer voxels than the large functional resting-state networks. The correlation analysis measures overlap and since our peak coordinates overlap only a small part of these networks, there are many voxels in the network not included. To provide more insight into the meaning of the spatial correlation results, we have therefore also provided the number of voxels of our peak coordinate clusters inside the network as a percentage of the total number of voxels of the clusters. Within our analysis, a reduced connectivity was found in the default mode network, auditory network and right fronto-parietal network when PD patients with $\mathrm{Cl}$ were compared with $\mathrm{HC}$. Furthermore, when comparing PD-Cl with $\mathrm{HC}$, we also noted a spatial correlation with the sensorimotor network, which could be related to the motor symptoms of patients with PD. For the comparison of PD-CU with PD-Cl, we detected a reduced connectivity specifically in the default mode network. After varying results in previous studies, our findings provide a more definite step in the differentiation of network disruptions associated with cognitive impairment in PD.

The default mode network is believed to serve an important role in various cognitive functions. It includes the medial parietal, bilateral inferior-lateral-parietal and ventromedial frontal cortex 
[69]. In healthy populations, reduced default mode network connectivity is associated with decreased memory performance, but also slower processing speed and decreased executive function [87-89]. In addition, alterations of the default mode network have been described in several other neurodegenerative disorders such as Alzheimer's disease, Huntington's disease and frontotemporal dementia [90-93]. Also in Parkinson's disease, changes in default mode network connectivity have been previously reported by several studies [94-96]. A recent metaanalysis of Tahmasian et al. (2017) investigated the resting-state functional connectivity in Parkinson's disease patients which were not selected specifically on the basis of cognitive performance. They similarly found an alteration in regions connected to the default mode network [97]. The authors concluded that this could be related to dysfunction of perception and executive functions in the PD patients. This supports our finding that the default mode network seems to be involved in cognitive decline in PD.

Besides the default mode network, we also found a notable decrease of functional connectivity in the auditory network of PD-Cl when compared to HC. This network consists of the superior temporal gyrus, Heschl's gyrus and the posterior insular region [69]. The auditory network is not as well studied as the default mode network in PD. So far, only one study has reported functional connectivity changes in this network in PD patients [98]. It has also been described that, compared to age matched controls, PD patients show greater difficulty in hearing spoken words [99]. Furthermore, a correlation between $\mathrm{Cl}$ and changes in auditory evoked potentials in PD has been reported by Nojszewska et al. (2009). The authors conclude that evaluation of the auditory evoked potentials may even serve as an indicator for $\mathrm{Cl}$ in PD [100]. Thus, although disruption of this network may not necessarily cause $\mathrm{Cl}$, these changes in the auditory network could point to hearing-loss as a potential risk factor for $\mathrm{Cl}$ in $\mathrm{PD}$ as has been suggested for dementia in the aging population [101]. However, only few studies have reported results about this network and in our meta-analysis this outcome was not preserved within the contrast of PD-CU with PD-Cl. Therefore, this outcome should be interpreted carefully. Based on our results it is also possible that disturbances in the auditory network are related to Parkinson's disease as a whole, but not specifically to cognitive impairment.

With respect to the fronto-parietal network, several studies have reported disruptions in the fronto-parietal network in PD patients with $\mathrm{Cl}[53,102,103]$. The fronto-parietal network seems to serve an important role in attention control [104]. While we hypothesized to find differences in functional connectivity of this network in PD-Cl as compared to HC and PD-CU, our meta-analysis did not show this as convincingly as expected. Only for the peak coordinates of the contrast $\mathrm{HC}$ vs. PD-Cl, a weak spatial correlation was found with this network. Since this association was not found for the PD-CU vs. PD-Cl contrast, while the connection with the default mode network became more explicit in this second comparison, a more significant role for the default mode network in cognitive impairment in PD is implicated.

Interestingly, the AES-SDM analysis also revealed patterns of increased functional connectivity when comparing PD-Cl patients with $\mathrm{HC}$, in particular in the right supramarginal gyrus. 
Furthermore, an increased connectivity was also found in the right cerebellum for the contrast of PD-Cl vs. PD-CU. It has been postulated that higher cortical functional connectivity in $\mathrm{PD}$ patients in the early stages of the disease, may reflect a compensatory mechanism to counteract slowly progressing $\mathrm{Cl}$ [105]. The ability of brain areas to display compensatory overactivation was first suggested by Reuter-Lorenz et al. (2008) [106]. Based upon PET CT observations, several studies have also reported such compensatory mechanisms in PD patients $[107,108]$. It has also been described that loss of compensatory hyperactivation is associated with a worse performance on cognitive tasks [109]. This hypothesis may form an explanation for the observed increased functional connectivity in this meta-analysis. However, it is important to note that our robustness analysis indicated that the peak coordinate in the right supramarginal gyrus was driven by the studies of Chen et al. (2017) and Madhyastha et al. (2015), while the increased connectivity in the right cerebellar area was based solely on the study of Chen et al. (2015) and Chen et al. (2017). Because both peak coordinates were driven by only two studies these results must be interpreted cautiously.

Given the consistent involvement of the default mode network, our results suggest this network may hold promise as a biomarker for $\mathrm{Cl}$ in PD patients, though further research is warranted. PD is thought to be a complex and heterogenic disease, probably with several different subtypes and changes over time [110,111]. For example, as stated in the introduction section, the dual syndrome hypothesis suggests that there are at least two PD subtypes, which among other things display a different profile of cognitive characteristics and a dissimilar degree of cognitive deterioration. [15]. This makes it more complex to determine a specific pattern of functional connectivity alterations in PD with $\mathrm{Cl}$ [112]. Therefore, deep phenotyping in longitudinal cohort studies, combining fMRI with other biomarkers such as structural imaging, genetic and clinical characteristics and cerebrospinal fluid biomarkers, would allow to further define specific fMRI correlates underlying the various phenotypes of the disease [113]. A number of studies performing multivariate analysis combining multi-modal neuroimaging techniques support the idea that Parkinson's disease is caused by a network-spread pathophysiology affecting several networks, including the default mode network, that correlate with cognitive deficits in PD [114-116]. Moreover, Long and colleagues (2012) developed a method which discriminated PD patients from $\mathrm{HC}$ with a power of $86.96 \%$ based on the combination of both structural and functional MRI characteristics [117]. The results of these studies support the promising future role of $\mathrm{fMRI}$ as a biomarker for PD.

As implicated by the multi-variate analysis described above, a relationship seems to exist between age-related brain atrophy in the healthy population and functional MRI activity of the brain [118]. For this reason, the application of brain atrophy corrections in fMRI studies in the PD population is important to avoid misleading results. However, in a considerable number of the studies included in our meta-analysis brain atrophy was not taken into account. Since our PD groups with cognitive decline showed a higher mean age when compared to the HC and PD-CU group, it cannot be ruled out that this might have influenced the meta-analysis to some extent. 
This study has several other limitations. First, the study is limited by the small to moderate amount of studies and a certain level of heterogeneity in study characteristics. As demonstrated in table 2, a variety of statistical or imaging methods, software packages and threshold settings were used in the included studies, which may have influenced our study results [119]. Specifically the inclusion of studies with different types of analytical methods (e.g. region of interest versus whole brain, or seed-based versus ICA) may introduce a bias in study results towards specific regions of interests. To provide more conclusive results, only studies applying a whole-brain analysis should be included in a meta-analysis like this. Our analysis could therefore be seen as an exploratory study which provides an indication of the results, while waiting for more conclusive results in the future. We suggest the validation of our findings with future studies consisting of an independent, methodologically homogeneous data set. Furthermore, sleeping during the acquisition has been shown to potentially increase functional connectivity in resting-state fMRI [120]. Unfortunately, only three of the included studies verified whether the subjects remained awake during the acquisition of the restingstate fMRI. Another major point of concern is that the fMRI was performed in 'ON' medication state as well as and in 'OFF' medication states among the studies included in the metaanalysis. Twelve studies completed the MRI procedure while patients were 'ON' medication, three studies performed the $\mathrm{FMRI}$ in 'OFF' status and three studies did not report if the patients were 'ON' or 'OFF' dopaminergic medication during the scanning procedure. Although most studies acquired the fMRI data after patients took their dopaminergic medication, this heterogeneity can still lead to misleading results, since dopaminergic medication influences brain connectivity patterns both in a linear and non-linear way [42, 121]. More specifically, this effect has also been described in the default mode network [122, 123]. Although these effects are particularly relevant for intra-individual differences we cannot rule out that the meta-analysis is influenced by this dissimilarity in study characteristics. For this reason, further studies with homogeneity regarding the dopaminergic status of the PD patients are necessary to validate our results. Finally, diversity in the definition of $\mathrm{MCl}$ is another concern and although agreement on the diagnostic criteria has been formulated [124-128] there is no consensus yet and the cut-off scores for mild cognitive impairment are still ranging between -1 SD and -2 SD. In this meta-analysis, different criteria were allowed for $\mathrm{MCl}$ in the included studies (Table 2). Unfortunately, due to the limited number of included articles, we were not able to perform further subgroup analysis.

In conclusion, this meta-analysis reveals specific resting-state network disruptions in PD patients with $\mathrm{Cl}$, especially in the default mode network. Quantification of these network connectivity changes could serve as a biomarker for $\mathrm{Cl}$ in PD and as such may be helpful in unravelling its pathophysiology. However, future studies with homogenous methodologically data sets, preferably combining different biomarkers in larger sample sizes are necessary to confirm these outcomes and to further explore the potential role of $\mathrm{fMRI}$ as a biomarker for $\mathrm{Cl}$. 


\section{ACKNOWLEDGMENTS}

This research did not receive any specific grant from funding agencies in the public, commercial, or not-for-profit sectors.

AW: Nothing to declare. SW: Nothing to declare. $\mathrm{HJ}$ : Heidi Jacobs is supported by funding from NWO (Veni - [451-14-035]), Alzheimer Nederland (fellowship [WE.15-2014-06]), a standard grant of the International Stichting voor Alzheimer Onderzoek [\#15007] and a research grant by the Deutsche Forschungsgemeinschaft [JA/2336/1-1]. AL: A.F.G. Leentjens received research grants from the Michael J Fox Foundation, as well as royalties from Reed-Elsevier, de Tijdstroom and Van Gorcum publishers. He receives payment from Elsevier Inc as Editor-in-Chief of the Journal of Psychsomatic Research. AD: A.A. Duits receives royalties from Hogrefe publishers. MK: Nothing to declare.

\section{AUTHOR CONTRIBUTIONS}

Conception and organization of the research project: AW, SW, AL, AD, HJ, MK. Execution of the search and article selection: AW, SW, HJ, MK. Design of the statistical analysis: AW, SW, HJ, MK. Execution of the statistical analysis: AW. Review and Critique of the statistical analysis: SW, HJ, MK. Writing of the first draft: AW. Review and critique on the manuscript: SW, HJ, AL, $A D, M K$. Final approval for submission: AW, SW, AL, AD, HJ, MK. 


\section{APPENDIX A. SUPPLEMENTARY DATA}

\section{S1. QUALITY ASSESSMENT}

Amboni et al. 2015

Baggio et al. 2015

Bezdicek et al. 2018

Borroni et al. 2015

Canu et al. 2015

Chen et al. 2015

Chen et al. 2017

Díez-Cirarda et al. 2018

Gorges et al. 2015

Hou et al. 2016

Madhyastha et al. 2015

Peraza et al. 2015

Peraza et al. 2017

Possin et al. 2013

Rektorova et al. 2012

Shin et al. 2016

Zhan et al. 2018

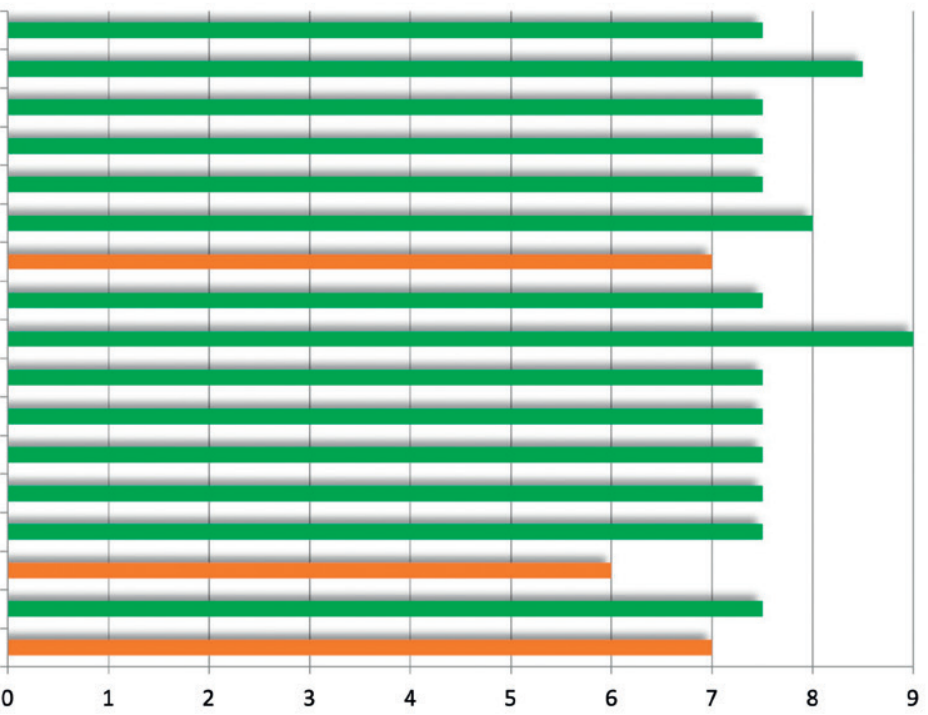

Figure S1. Quality assessment derived from the guidelines of Poldrack et al. [28]. Total score was based on 9 criteria. For each item $0,0.5$ or 1 point could be scored. An overall score of $\geq 7.5$ was considered as good (green), 4-7.5 as fair (orange) and $\leq 4$ as poor (red) quality.

\section{S2. ROBUSTNESS ANALYSIS}

\section{Contrast $\mathrm{HC}$ vs. PD-CI}

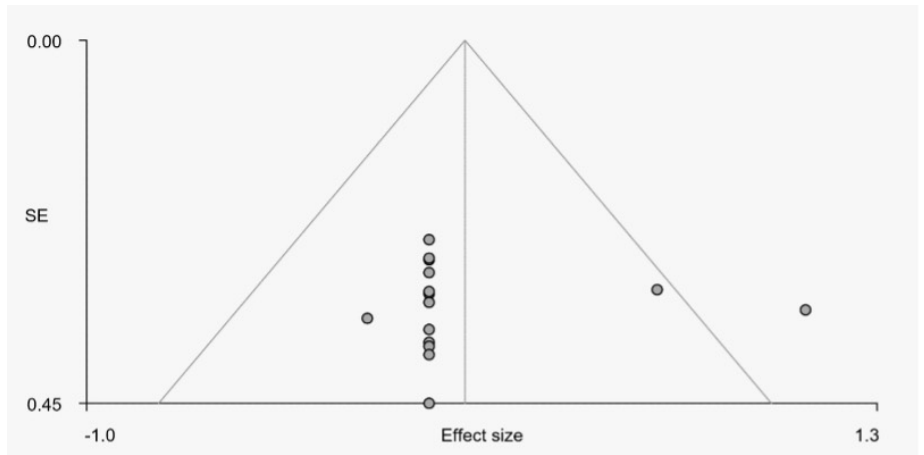

Figure S2.1. Right Supramarginal gyrus, BA 40, Bias: 0.25 , t: $0.14, d f: 13, p: 0.887$ 
40 CHAPTER 2

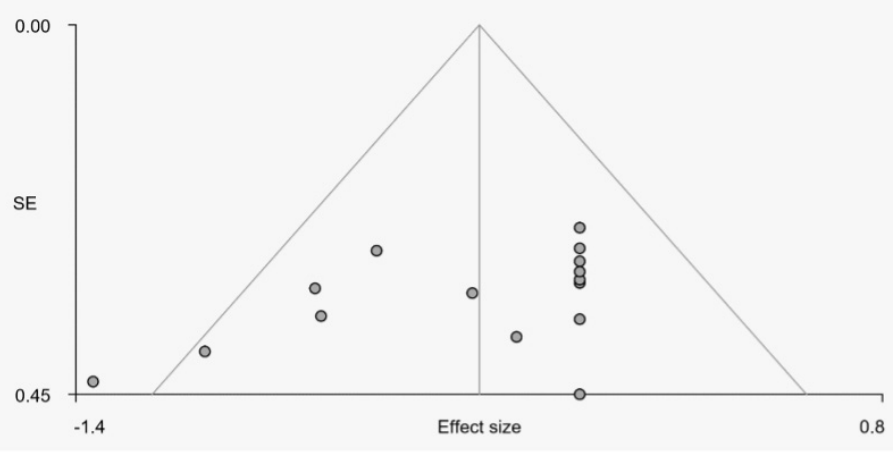

Figure S2.2. Right rolandic operculum (BA 48), Bias: -3.39, t: -1.97, df: 13, p: 0.070

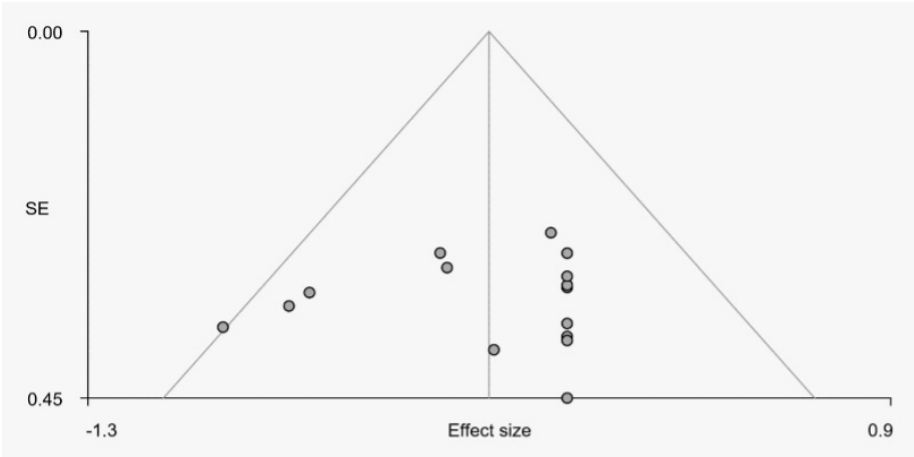

Figure S2.3. Left inferior parietal gyri (BA 40), Bias: -0.65, t: -0.39, df: 13, p: 0.701

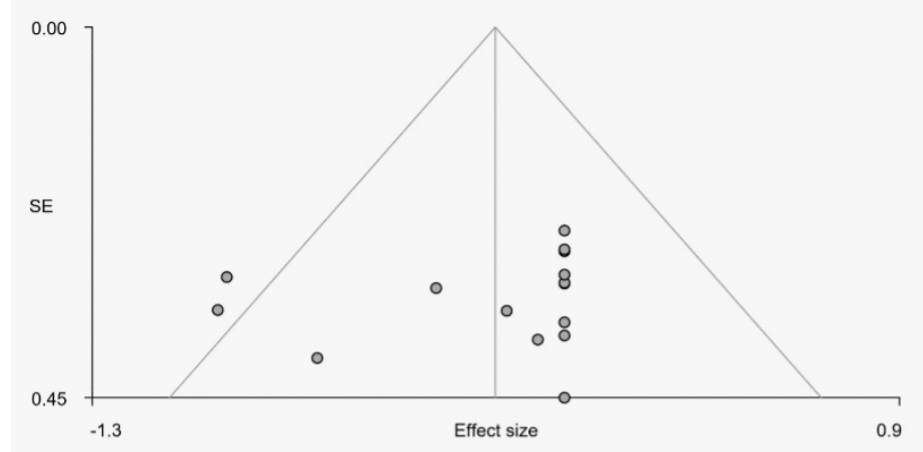

Figure S2.4. Right angular gyrus (BA 39), Bias: $-1.43, t:-0.79, d f: 13, p: 0.442$ 


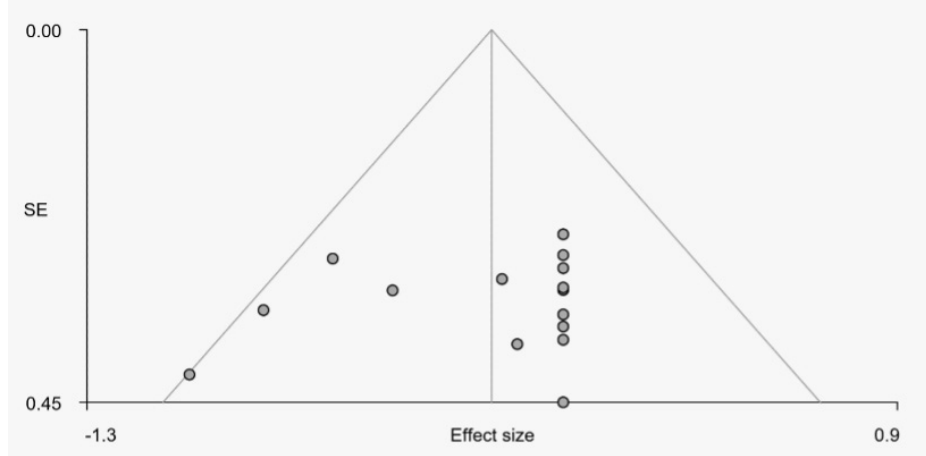

Figure S2.5. Left parahippocampal gyrus (BA 37), Bias: -1.20, t: -0.72, df: 13, p: 0.485

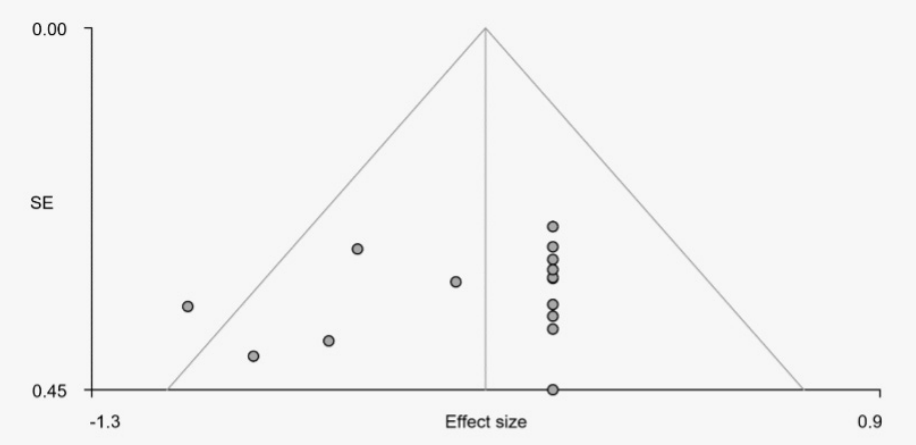

Figure S2.6. Right calcarine fissure/surrounding cortex (BA 23), Bias: -1.94, t: -1.16, df: 13, p: 0.267

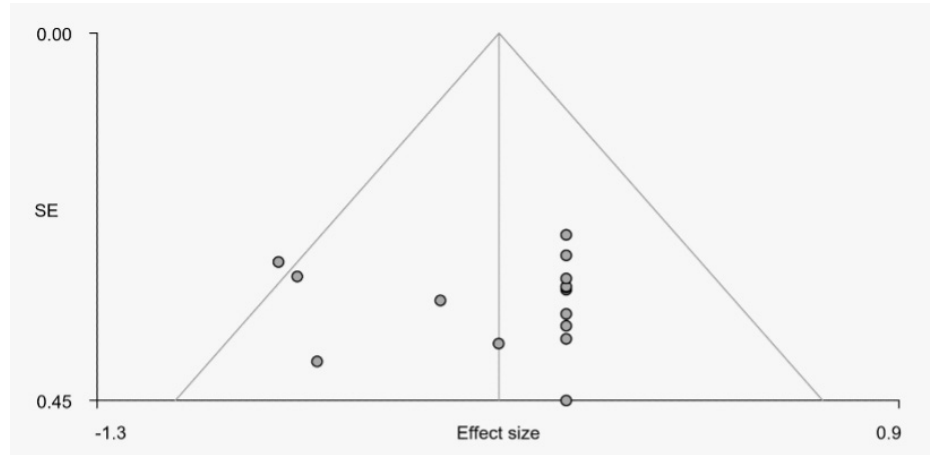

Figure S2.7. Right superior frontal gyrus, dorsolateral (BA 9), Bias: 0.11, t: 0.06, df: 13, p: 0.952 
$42 \mid$ CHAPTER 2

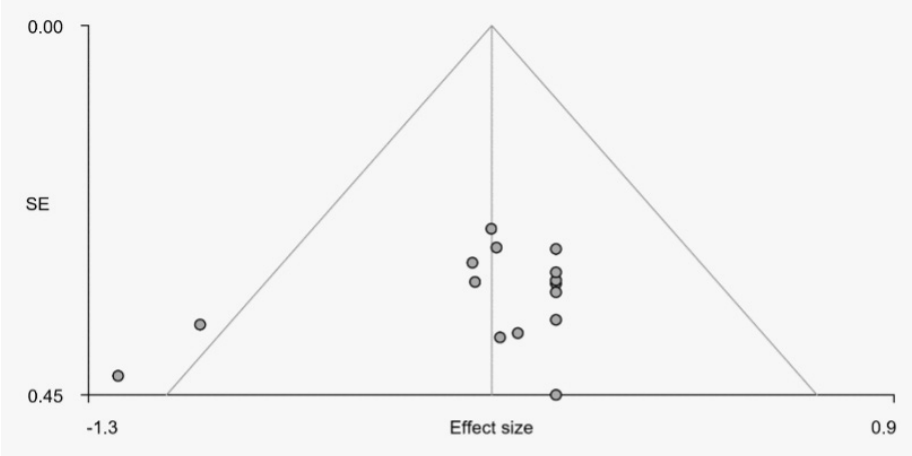

Figure S2.8. Right precentral gyrus (BA 4), Bias: -2.11, t: -1.44, df: $13, p: 0.174$

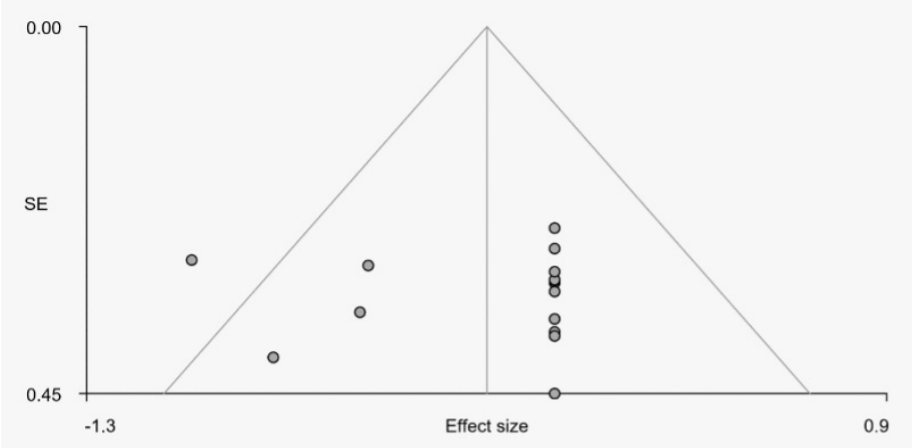

Figure S2.9. Right precentral gyrus (BA 6), Bias: -0.14, t: -0.08, df: 13, p: 0.940

\section{Contrast PD-CI vs. PD CU}

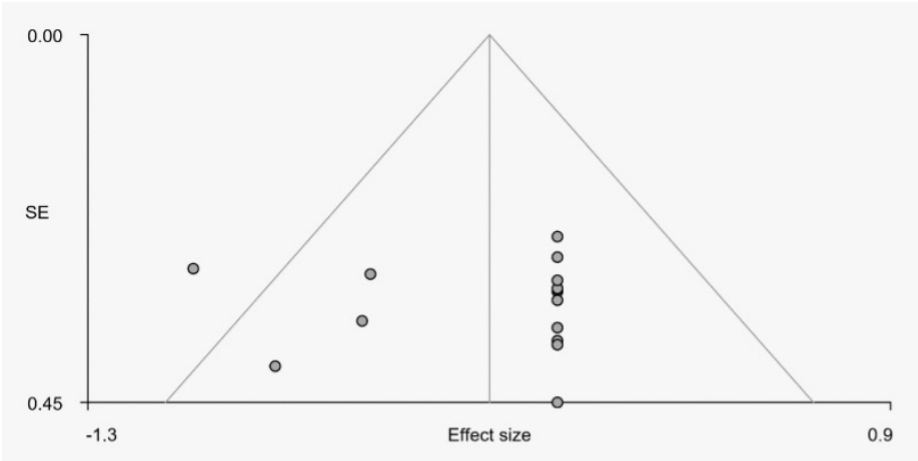

Figure S2.10. Right cerebellum, hemispheric lobule VI (BA 37), Bias: 2.15, t: 1.35, df: 7, p: 0.221 


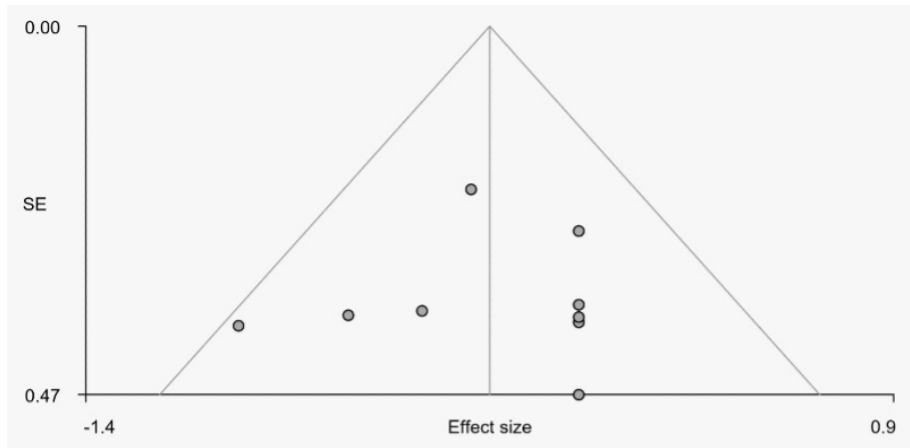

Figure S2.11. Left precuneus, Bias: $-0.27, t:-0.20, d f: 7, p: 0.849$

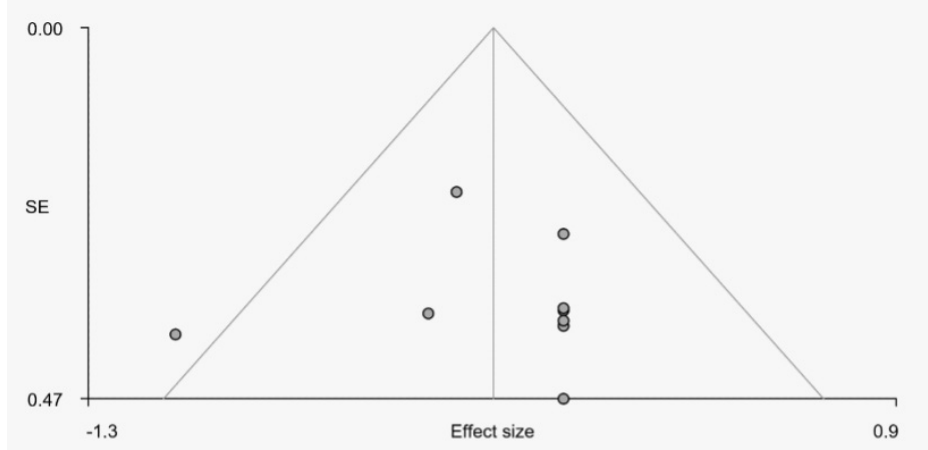

Figure S2.12. Right median cingulate / paracingulate gyri (BA 24), Bias: 0.06, t: 0.04, df: 7, p: 0.967

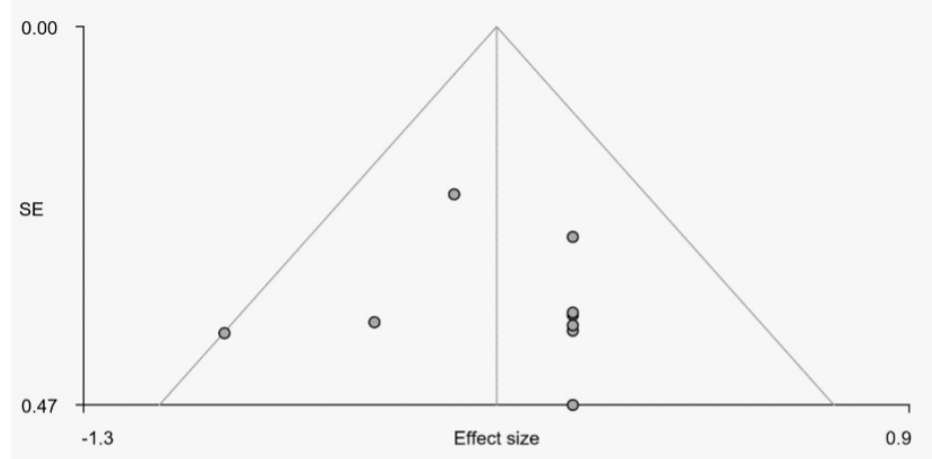

Figure S2.13. Left superior frontal gyrus, dorsolateral (BA 10), Bias: 0.21, t: 0.15, df: 7, p: 0.882 


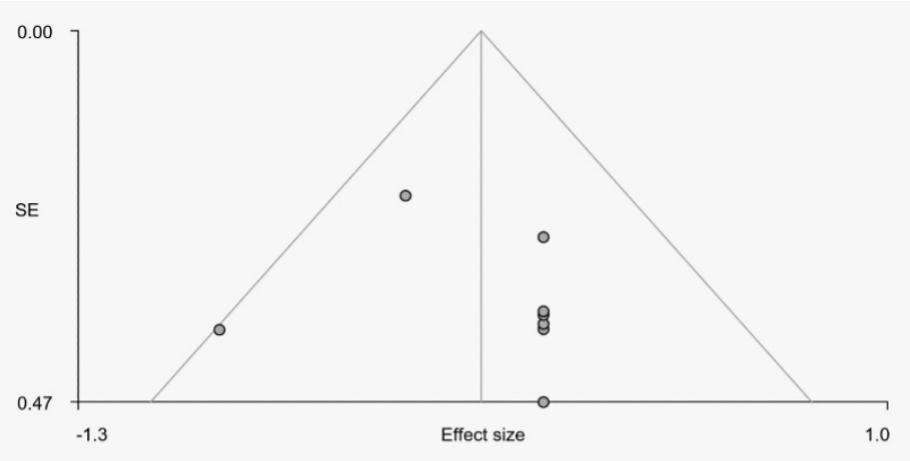

Figure S2.14. Right precentral gyrus (BA 4), Bias: 0.93, t: 0.74, df: 7, p: 0.486

\section{S3. Spatial correlation analysis}

\begin{tabular}{|c|c|c|c|c|}
\hline $\begin{array}{l}\text { Resting-state networks } \\
\text { Smith et al. (2009) }\end{array}$ & $\mathrm{HC}>\mathrm{PD}-\mathrm{Cl}$ & $\mathrm{HC}<\mathrm{PD}-\mathrm{Cl}$ & PD-CU > PD-Cl & PD-CU $<$ PD-Cl \\
\hline Medial visual network & 0.03 & 0.02 & 0.03 & 0.04 \\
\hline Occipital visual network & 0.01 & 0.01 & 0.01 & 0.04 \\
\hline Lateral visual network & 0.00 & 0.03 & 0.02 & 0.05 \\
\hline Default mode network & 0.07 & 0.01 & 0.24 & 0.02 \\
\hline Cerebellum & 0.01 & 0.00 & 0.02 & 0.19 \\
\hline Sensorimotor network & 0.13 & 0.05 & 0.00 & 0.01 \\
\hline Auditory network & 0.13 & 0.01 & 0.02 & 0.04 \\
\hline Executive control network & 0.03 & 0.01 & 0.02 & 0.00 \\
\hline Right frontoparietal network & 0.07 & 0.05 & 0.04 & 0.03 \\
\hline Left frontoparietal network & 0.00 & 0.01 & 0.01 & 0.00 \\
\hline
\end{tabular}

Figure S3. Results of the FSL correlation analysis between AES-SDM peak coordinates and ten resting-state network templates described by Smith et al. (2009). Spatial correlation values ( $r$ ) are given for each network. All images in MNI-space, with a voxel size of $2 \times 2 \mathrm{~mm}$. 


\section{REFERENCES}

1. J. Jankovic, Parkinson's disease: clinical features and diagnosis, Journal of neurology, neurosurgery, and psychiatry 79(4) (2008) 368-76.

2. A.M. Al-Radaideh, E.M. Rababah, The role of magnetic resonance imaging in the diagnosis of Parkinson's disease: a review, Clinical imaging 40(5) (2016) 987-996.

3. R.B. Postuma, D. Berg, M. Stern, W. Poewe, C.W. Olanow, W. Oertel, J. Obeso, K. Marek, I. Litvan, A.E. Lang, G. Halliday, C.G. Goetz, T. Gasser, B. Dubois, P. Chan, B.R. Bloem, C.H. Adler, G. Deuschl, MDS clinical diagnostic criteria for Parkinson's disease, Mov Disord 30(12) (2015) 1591-601.

4. A.J. Yarnall, D.P. Breen, G.W. Duncan, T.K. Khoo, S.Y. Coleman, M.J. Firbank, C. Nombela, S. Winder-Rhodes, J.R. Evans, J.B. Rowe, B. Mollenhauer, N. Kruse, G. Hudson, P.F. Chinnery, J.T. O’Brien, T.W. Robbins, K. Wesnes, D.J. Brooks, R.A. Barker, D.J. Burn, I.-P.S. Group, Characterizing mild cognitive impairment in incident Parkinson disease: the ICICLE-PD study, Neurology 82(4) (2014) 308-16.

5. P. Svenningsson, E. Westman, C. Ballard, D. Aarsland, Cognitive impairment in patients with Parkinson's disease: diagnosis, biomarkers, and treatment, The Lancet. Neurology 11(8) (2012) 697-707.

6. M.A. Hely, W.G. Reid, M.A. Adena, G.M. Halliday, J.G. Morris, The Sydney multicenter study of Parkinson's disease: the inevitability of dementia at 20 years, Movement disorders : official journal of the Movement Disorder Society 23(6) (2008) 837-44.

7. C.C. Janvin, J.P. Larsen, D. Aarsland, K. Hugdahl, Subtypes of mild cognitive impairment in Parkinson's disease: progression to dementia, Movement disorders : official journal of the Movement Disorder Society 21(9) (2006) 1343-9.

8. M. Delgado-Alvarado, B. Gago, I. Navalpotro-Gomez, H. Jimenez-Urbieta, M.C. Rodriguez-Oroz, Biomarkers for dementia and mild cognitive impairment in Parkinson's disease, Movement disorders : official journal of the Movement Disorder Society (2016).

9. M.G. Spillantini, M.L. Schmidt, V.M. Lee, J.Q. Trojanowski, R. Jakes, M. Goedert, Alpha-synuclein in Lewy bodies, Nature 388(6645) (1997) 839-40.

10. R.E. Skogseth, K. Bronnick, J.B. Pereira, B. Mollenhauer, D. Weintraub, T. Fladby, D. Aarsland, Associations between Cerebrospinal Fluid Biomarkers and Cognition in Early Untreated Parkinson's Disease, Journal of Parkinson's disease 5(4) (2015) 783-92.

11. D. Aarsland, R. Perry, A. Brown, J.P. Larsen, C. Ballard, Neuropathology of dementia in Parkinson's disease: a prospective, community-based study, Annals of neurology 58(5) (2005) 773-6.

12. G.M. Halliday, J.B. Leverenz, J.S. Schneider, C.H. Adler, The neurobiological basis of cognitive impairment in Parkinson's disease, Movement disorders : official journal of the Movement Disorder Society 29(5) (2014) 634-50.

13. M. Petrou, N.I. Bohnen, M.L. Muller, R.A. Koeppe, R.L. Albin, K.A. Frey, Abeta-amyloid deposition in patients with Parkinson disease at risk for development of dementia, Neurology 79(11) (2012) 1161-7. 
14. Y. Compta, L. Parkkinen, S.S. O'Sullivan, J. Vandrovcova, J.L. Holton, C. Collins, T. Lashley, C. Kallis, D.R. Williams, R. de Silva, A.J. Lees, T. Revesz, Lewy- and Alzheimer-type pathologies in Parkinson's disease dementia: which is more important?, Brain : a journal of neurology 134(Pt 5) (2011) 1493-1505.

15. A.A. Kehagia, R.A. Barker, T.W. Robbins, Cognitive impairment in Parkinson's disease: the dual syndrome hypothesis, Neurodegener Dis 11(2) (2013) 79-92.

16. R. Cash, T. Dennis, R. L'Heureux, R. Raisman, F. Javoy-Agid, B. Scatton, Parkinson's disease and dementia: norepinephrine and dopamine in locus ceruleus, Neurology 37(1) (1987) 42-6.

17. V. Chan-Palay, E. Asan, Alterations in catecholamine neurons of the locus coeruleus in senile dementia of the Alzheimer type and in Parkinson's disease with and without dementia and depression, J Comp Neurol 287(3) (1989) 373-92.

18. C. Zarow, S.A. Lyness, J.A. Mortimer, H.C. Chui, Neuronal loss is greater in the locus coeruleus than nucleus basalis and substantia nigra in Alzheimer and Parkinson diseases, Archives of neurology 60 (3) (2003) 337-41.

19. D.J. Brooks, P. Piccini, Imaging in Parkinson's disease: the role of monoamines in behavior, Biol Psychiatry 59(10) (2006) 908-18.

20. B. Scatton, F. Javoy-Agid, L. Rouquier, B. Dubois, Y. Agid, Reduction of cortical dopamine, noradrenaline, serotonin and their metabolites in Parkinson's disease, Brain research 275(2) (1983) 321-8.

21. K.A. Jellinger, Pathology of Parkinson's disease. Changes other than the nigrostriatal pathway, Mol Chem Neuropathol 14(3) (1991) 153-97.

22. A.M. Gotham, R.G. Brown, C.D. Marsden, 'Frontal' cognitive function in patients with Parkinson's disease ‘on' and 'off' levodopa, Brain : a journal of neurology 111 ( Pt 2) (1988) 299-321.

23. R. Cools, R.A. Barker, B.J. Sahakian, T.W. Robbins, Enhanced or impaired cognitive function in Parkinson's disease as a function of dopaminergic medication and task demands, Cerebral cortex (New York, N.Y. : 1991) 11(12) (2001) 1136-43.

24. R. Cools, R.A. Barker, B.J. Sahakian, T.W. Robbins, L-Dopa medication remediates cognitive inflexibility, but increases impulsivity in patients with Parkinson's disease, Neuropsychologia 41(11) (2003) 1431-41.

25. R. Swainson, R.D. Rogers, B.J. Sahakian, B.A. Summers, C.E. Polkey, T.W. Robbins, Probabilistic learning and reversal deficits in patients with Parkinson's disease or frontal or temporal lobe lesions: possible adverse effects of dopaminergic medication, Neuropsychologia 38(5) (2000) 596-612.

26. C.H. Williams-Gray, A. Goris, M. Saiki, T. Foltynie, D.A. Compston, S.J. Sawcer, R.A. Barker, Apolipoprotein E genotype as a risk factor for susceptibility to and dementia in Parkinson's disease, Journal of neurology 256(3) (2009) 493-8.

27. J.F. Morley, S.X. Xie, H.I. Hurtig, M.B. Stern, A. Colcher, S. Horn, N. Dahodwala, J.E. Duda, D. Weintraub, A.S. Chen-Plotkin, V. Van Deerlin, D. Falcone, A. Siderowf, Genetic influences on cognitive decline in Parkinson's disease, Movement disorders : official journal of the Movement Disorder Society 27(4) (2012) 512-8. 
28. B.S. Harhangi, M.C. de Rijk, C.M. van Duijn, C. Van Broeckhoven, A. Hofman, M.M. Breteler, APOE and the risk of PD with or without dementia in a population-based study, Neurology 54(6) (2000) 1272-6.

29. L.M. de Lau, C.M. Schipper, A. Hofman, P.J. Koudstaal, M.M. Breteler, Prognosis of Parkinson disease: risk of dementia and mortality: the Rotterdam Study, Archives of neurology 62(8) (2005) 1265-9.

30. C.H. Williams-Gray, J.R. Evans, A. Goris, T. Foltynie, M. Ban, T.W. Robbins, C. Brayne, B.S. Kolachana, D.R. Weinberger, S.J. Sawcer, R.A. Barker, The distinct cognitive syndromes of Parkinson's disease: 5 year follow-up of the CamPaIGN cohort, Brain : a journal of neurology 132(Pt 11) (2009) 2958-69.

31. N. Seto-Salvia, J. Pagonabarraga, H. Houlden, B. Pascual-Sedano, O. Dols-Icardo, A. Tucci, C. Paisan-Ruiz, A. Campolongo, S. Anton-Aguirre, I. Martin, L. Munoz, E. Bufill, L. Vilageliu, D. Grinberg, M. Cozar, R. Blesa, A. Lleo, J. Hardy, J. Kulisevsky, J. Clarimon, Glucocerebrosidase mutations confer a greater risk of dementia during Parkinson's disease course, Movement disorders : official journal of the Movement Disorder Society 27(3) (2012) 393-9.

32. R.N. Alcalay, E. Caccappolo, H. Mejia-Santana, M. Tang, L. Rosado, M. Orbe Reilly, D. Ruiz, B. Ross, M. Verbitsky, S. Kisselev, E. Louis, C. Comella, A. Colcher, D. Jennings, M. Nance, S. Bressman, W.K. Scott, C. Tanner, S. Mickel, H. Andrews, C. Waters, S. Fahn, L. Cote, S. Frucht, B. Ford, M. Rezak, K. Novak, J.H. Friedman, R. Pfeiffer, L. Marsh, B. Hiner, A. Siderowf, H. Payami, E. Molho, S. Factor, R. Ottman, L.N. Clark, K. Marder, Cognitive performance of GBA mutation carriers with early-onset PD: the CORE-PD study, Neurology 78(18) (2012) 1434-40.

33. K. Brockmann, K. Srulijes, S. Pflederer, A.K. Hauser, C. Schulte, W. Maetzler, T. Gasser, D. Berg, GBA-associated Parkinson's disease: reduced survival and more rapid progression in a prospective longitudinal study, Movement disorders: official journal of the Movement Disorder Society 30(3) (2015) 407-11.

34. S.E. Winder-Rhodes, A. Hampshire, J.B. Rowe, J.E. Peelle, T.W. Robbins, A.M. Owen, R.A. Barker, Association between MAPT haplotype and memory function in patients with Parkinson's disease and healthy aging individuals, Neurobiology of aging 36(3) (2015) 1519-1528.

35. M. Pievani, W. de Haan, T. Wu, W.W. Seeley, G.B. Frisoni, Functional network disruption in the degenerative dementias, The Lancet. Neurology 10(9) (2011) 829-43.

36. B.B. Biswal, M. Mennes, X.N. Zuo, S. Gohel, C. Kelly, S.M. Smith, C.F. Beckmann, J.S. Adelstein, R.L. Buckner, S. Colcombe, A.M. Dogonowski, M. Ernst, D. Fair, M. Hampson, M.J. Hoptman, J.S. Hyde, V.J. Kiviniemi, R. Kotter, S.J. Li, C.P. Lin, M.J. Lowe, C. Mackay, D.J. Madden, K.H. Madsen, D.S. Margulies, H.S. Mayberg, K. McMahon, C.S. Monk, S.H. Mostofsky, B.J. Nagel, J.J. Pekar, S.J. Peltier, S.E. Petersen, V. Riedl, S.A. Rombouts, B. Rypma, B.L. Schlaggar, S. Schmidt, R.D. Seidler, G.J. Siegle, C. Sorg, G.J. Teng, J. Veijola, A. Villringer, M. Walter, L. Wang, X.C. Weng, S. Whitfield-Gabrieli, P. Williamson, C. Windischberger, Y.F. Zang, H.Y. Zhang, F.X. Castellanos, M.P. Milham, Toward discovery science of human brain function, Proceedings of the National Academy of Sciences of the United States of America 107(10) (2010) 4734-9.

37. M.P. van den Heuvel, H.E. Hulshoff Pol, Exploring the brain network: a review on resting-state fMRI functional connectivity, European neuropsychopharmacology : the journal of the European College of Neuropsychopharmacology 20(8) (2010) 519-34.

38. J. Pa, A.S. Berry, M. Compagnone, J. Boccanfuso, I. Greenhouse, M.T. Rubens, J.K. Johnson, A. Gazzaley, Cholinergic enhancement of functional networks in older adults with mild cognitive impairment, Annals of neurology 73(6) (2013) 762-73. 
39. D. Shah, I. Blockx, P.J. Guns, P.P. De Deyn, D. Van Dam, E. Jonckers, Y.P.R. Delgado, M. Verhoye, A. Van der Linden, Acute modulation of the cholinergic system in the mouse brain detected by pharmacological resting-state functional MRI, Neurolmage 109 (2015) 151-9.

40. H. Zheng, K. Onoda, Y. Wada, S. Mitaki, T. Nabika, S. Yamaguchi, Serotonin-1A receptor C-1019G polymorphism affects brain functional networks, Sci Rep 7(1) (2017) 12536.

41. B.L. Klaassens, H.C. van Gorsel, N. Khalili-Mahani, J. van der Grond, B.T. Wyman, B. Whitcher, S.A. Rombouts, J.M. van Gerven, Single-dose serotonergic stimulation shows widespread effects on functional brain connectivity, Neurolmage 122 (2015) 440-50.

42. D.M. Cole, C.F. Beckmann, N.Y. Oei, S. Both, J.M. van Gerven, S.A. Rombouts, Differential and distributed effects of dopamine neuromodulations on resting-state network connectivity, Neurolmage 78 (2013) 59-67.

43. W.C. Lin, H.L. Chen, T.W. Hsu, C.C. Hsu, Y.C. Huang, N.W. Tsai, C.H. Lu, Correlation between Dopamine Transporter Degradation and Striatocortical Network Alteration in Parkinson's Disease, Front Neurol 8 (2017) 323.

44. A. Dagher, A. Nagano-Saito, Functional and anatomical magnetic resonance imaging in Parkinson's disease, Molecular Imaging and Biology 9(4) (2007) 234-242.

45. M.C. Campbell, J.M. Koller, A.Z. Snyder, C. Buddhala, P.T. Kotzbauer, J.S. Perlmutter, CSF proteins and resting-state functional connectivity in Parkinson disease, Neurology 84(24) (2015) 2413-21.

46. B. Biswal, F.Z. Yetkin, V.M. Haughton, J.S. Hyde, Functional connectivity in the motor cortex of resting human brain using echo-planar MRI, Magnetic resonance in medicine 34(4) (1995) 537-41.

47. D.A. Gusnard, M.E. Raichle, M.E. Raichle, Searching for a baseline: functional imaging and the resting human brain, Nature reviews. Neuroscience 2(10) (2001) 685-94.

48. T.M. Madhyastha, M.K. Askren, J. Zhang, J.B. Leverenz, T.J. Montine, T.J. Grabowski, Group comparison of spatiotemporal dynamics of intrinsic networks in Parkinson's disease, Brain : a journal of neurology 138(Pt 9) (2015) 2672-86.

49. I. Rektorova, L. Krajcovicova, R. Marecek, M. Mikl, Default mode network and extrastriate visual resting state network in patients with Parkinson's disease dementia, Neuro-degenerative diseases 10(1-4) (2012) 232-7.

50. T. van Eimeren, O. Monchi, B. Ballanger, A.P. Strafella, Dysfunction of the default mode network in Parkinson disease: a functional magnetic resonance imaging study, Archives of neurology 66(7) (2009) 877-83.

51. S. Tinaz, H.E. Schendan, C.E. Stern, Fronto-striatal deficit in Parkinson's disease during semantic event sequencing, Neurobiology of aging 29(3) (2008) 397-407.

52. M. Gorges, H.P. Muller, D. Lule, E.H. Pinkhardt, A.C. Ludolph, J. Kassubek, To rise and to fall: functional connectivity in cognitively normal and cognitively impaired patients with Parkinson's disease, Neurobiology of aging 36(4) (2015) 1727-35. 
53. A.V. Lebedev, E. Westman, A. Simmons, A. Lebedeva, F.J. Siepel, J.B. Pereira, D. Aarsland, Largescale resting state network correlates of cognitive impairment in Parkinson's disease and related dopaminergic deficits, Frontiers in systems neuroscience 8 (2014) 45.

54. I. Rektorova, L. Krajcovicova, R. Marecek, M. Mikl, Effective connectivity of the default mode network in parkinson's disease and parkinson's disease dementia, Clinical Neurophysiology 125 (2014) S126-S127.

55. A.R. Laird, P.M. Fox, S.B. Eickhoff, J.A. Turner, K.L. Ray, D.R. McKay, D.C. Glahn, C.F. Beckmann, S.M. Smith, P.T. Fox, Behavioral interpretations of intrinsic connectivity networks, Journal of cognitive neuroscience 23(12) (2011) 4022-37.

56. A. Abos, H.C. Baggio, B. Segura, A.I. Garcia-Diaz, Y. Compta, M.J. Marti, F. Valldeoriola, C. Junque, Discriminating cognitive status in Parkinson's disease through functional connectomics and machine learning, Sci Rep 7 (2017) 45347.

57. M. Mijalkov, E. Kakaei, J.B. Pereira, E. Westman, G. Volpe, I. Alzheimer's Disease Neuroimaging, BRAPH: A graph theory software for the analysis of brain connectivity, PloS one 12(8) (2017) e0178798.

58. H.C. Baggio, R. Sala-Llonch, B. Segura, M.J. Marti, F. Valldeoriola, Y. Compta, E. Tolosa, C. Junque, Functional brain networks and cognitive deficits in Parkinson's disease, Human brain mapping 35(9) (2014) 4620-34.

59. R.A. Poldrack, P.C. Fletcher, R.N. Henson, K.J. Worsley, M. Brett, T.E. Nichols, Guidelines for reporting an fMRI study, Neurolmage 40(2) (2008) 409-14.

60. J. Radua, D. Mataix-Cols, M.L. Phillips, W. El-Hage, D.M. Kronhaus, N. Cardoner, S. Surguladze, A new meta-analytic method for neuroimaging studies that combines reported peak coordinates and statistical parametric maps, European psychiatry : the journal of the Association of European Psychiatrists 27(8) (2012) 605-11.

61. J. Radua, D. Mataix-Cols, Meta-analytic methods for neuroimaging data explained, Biology of mood \& anxiety disorders 2 (2012) 6 .

62. H.I. Jacobs, J. Radua, H.C. Luckmann, A.T. Sack, Meta-analysis of functional network alterations in Alzheimer's disease: toward a network biomarker, Neuroscience and biobehavioral reviews 37(5) (2013) 753-65.

63. D. Cooper, V. Barker, J. Radua, P. Fusar-Poli, S.M. Lawrie, Multimodal voxel-based meta-analysis of structural and functional magnetic resonance imaging studies in those at elevated genetic risk of developing schizophrenia, Psychiatry research 221(1) (2014) 69-77.

64. H. Hart, J. Radua, T. Nakao, D. Mataix-Cols, K. Rubia, Meta-analysis of functional magnetic resonance imaging studies of inhibition and attention in attention-deficit/hyperactivity disorder: exploring task-specific, stimulant medication, and age effects, JAMA psychiatry 70(2) (2013) 185-98.

65. Y.J. Meng, W. Deng, H.Y. Wang, W.J. Guo, T. Li, C. Lam, X. Lin, Reward pathway dysfunction in gambling disorder: A meta-analysis of functional magnetic resonance imaging studies, Behavioural brain research 275 (2014) 243-51.

66. D. Shen, L. Cui, J. Fang, B. Cui, D. Li, H. Tai, Voxel-Wise Meta-Analysis of Gray Matter Changes in Amyotrophic Lateral Sclerosis, Frontiers in aging neuroscience 8 (2016) 64. 
67. X. Yang, F. Tian, H. Zhang, J. Zeng, T. Chen, S. Wang, Z. Jia, Q. Gong, Cortical and subcortical gray matter shrinkage in alcohol-use disorders: a voxel-based meta-analysis, Neuroscience and biobehavioral reviews 66 (2016) 92-103.

68. J. Radua, D. Mataix-Cols, Voxel-wise meta-analysis of grey matter changes in obsessive-compulsive disorder, The British journal of psychiatry : the journal of mental science 195(5) (2009) 393-402.

69. S.M. Smith, P.T. Fox, K.L. Miller, D.C. Glahn, P.M. Fox, C.E. Mackay, N. Filippini, K.E. Watkins, R. Toro, A.R. Laird, C.F. Beckmann, Correspondence of the brain's functional architecture during activation and rest, Proceedings of the National Academy of Sciences of the United States of America 106(31) (2009) 13040-5.

70. P.T. Fox, J.L. Lancaster, Opinion: Mapping context and content: the BrainMap model, Nature reviews. Neuroscience 3(4) (2002) 319-21.

71. P.T. Fox, A.R. Laird, S.P. Fox, P.M. Fox, A.M. Uecker, M. Crank, S.F. Koenig, J.L. Lancaster, BrainMap taxonomy of experimental design: description and evaluation, Human brain mapping 25(1) (2005) 185-98.

72. A.R. Laird, J.L. Lancaster, P.T. Fox, BrainMap: the social evolution of a human brain mapping database, Neuroinformatics 3(1) (2005) 65-78.

73. M. Amboni, A. Tessitore, F. Esposito, G. Santangelo, M. Picillo, C. Vitale, A. Giordano, R. Erro, R. de Micco, D. Corbo, G. Tedeschi, P. Barone, Resting-state functional connectivity associated with mild cognitive impairment in Parkinson's disease, Journal of neurology 262(2) (2015) 425-34.

74. K.L. Possin, G.A. Kang, C. Guo, E.M. Fine, A.J. Trujillo, C.A. Racine, R. Wilheim, E.T. Johnson, J.L. Witt, W.W. Seeley, B.L. Miller, J.H. Kramer, Rivastigmine is associated with restoration of left frontal brain activity in Parkinson's disease, Movement disorders : official journal of the Movement Disorder Society 28(10) (2013) 1384-90.

75. H.C. Baggio, B. Segura, R. Sala-Llonch, M.J. Marti, F. Valldeoriola, Y. Compta, E. Tolosa, C. Junque, Cognitive impairment and resting-state network connectivity in Parkinson's disease, Human brain mapping 36(1) (2015) 199-212.

76. E. Canu, F. Agosta, E. Sarasso, M.A. Volonte, L. Sarro, S. Galantucci, S. Basaia, R. Gatti, A. Falini, G. Comi, M. Filippi, Brain structural and functional abnormalities in Parkinson's disease patients with freezing of gait, European journal of neurology 22 (2015) 295.

77. B. Borroni, E. Premi, A. Formenti, R. Turrone, A. Alberici, E. Cottini, C. Rizzetti, R. Gasparotti, A. Padovani, Structural and functional imaging study in dementia with Lewy bodies and Parkinson's disease dementia, Parkinsonism \& related disorders 21(9) (2015) 1049-55.

78. B. Chen, G.G. Fan, H. Liu, S. Wang, Changes in anatomical and functional connectivity of Parkinson's disease patients according to cognitive status, European journal of radiology 84(7) (2015) 1318-24.

79. L.R. Peraza, S.J. Colloby, M.J. Firbank, G.S. Greasy, I.G. McKeith, M. Kaiser, J. O’Brien, J.P. Taylor, Resting state in Parkinson's disease dementia and dementia with Lewy bodies: commonalities and differences, International journal of geriatric psychiatry 30(11) (2015) 1135-46.

80. Y. Hou, J. Yang, C. Luo, W. Song, R. Ou, W. Liu, Q. Gong, H. Shang, Dysfunction of the Default Mode Network in Drug-Naive Parkinson's Disease with Mild Cognitive Impairments: A Resting-State fMRI Study, Frontiers in aging neuroscience 8 (2016) 247. 
81. L.R. Peraza, D. Nesbitt, R.A. Lawson, G.W. Duncan, A.J. Yarnall, T.K. Khoo, M. Kaiser, M.J. Firbank, J.T. O’Brien, R.A. Barker, D.J. Brooks, D.J. Burn, J.P. Taylor, Intra- and inter-network functional alterations in Parkinson's disease with mild cognitive impairment, Human brain mapping 38(3) (2017) 1702-1715.

82. O. Bezdicek, T. Ballarini, F. Ruzicka, J. Roth, K. Mueller, R. Jech, M.L. Schroeter, Mild cognitive impairment disrupts attention network connectivity in Parkinson's disease: A combined multimodal MRI and meta-analytical study, Neuropsychologia 112 (2018) 105-115.

83. B. Chen, S. Wang, W. Sun, X. Shang, H. Liu, G. Liu, J. Gao, G. Fan, Functional and structural changes in gray matter of parkinson's disease patients with mild cognitive impairment, European journal of radiology 93 (2017) 16-23.

84. M. Diez-Cirarda, A.P. Strafella, J. Kim, J. Pena, N. Ojeda, A. Cabrera-Zubizarreta, N. Ibarretxe-Bilbao, Dynamic functional connectivity in Parkinson's disease patients with mild cognitive impairment and normal cognition, Neurolmage. Clinical 17 (2018) 847-855.

85. Z.W. Zhan, L.Z. Lin, E.H. Yu, J.W. Xin, L. Lin, H.L. Lin, Q.Y. Ye, X.C. Chen, X.D. Pan, Abnormal resting-state functional connectivity in posterior cingulate cortex of Parkinson's disease with mild cognitive impairment and dementia, CNS neuroscience \& therapeutics (2018).

86. N.Y. Shin, Y.S. Shin, P.H. Lee, U. Yoon, S. Han, D.J. Kim, S.K. Lee, Different Functional and Microstructural Changes Depending on Duration of Mild Cognitive Impairment in Parkinson Disease, AJNR. American journal of neuroradiology 37(5) (2016) 897-903.

87. D. Vidal-Pineiro, C. Valls-Pedret, S. Fernandez-Cabello, E.M. Arenaza-Urquijo, R. Sala-Llonch, E. Solana, N. Bargallo, C. Junque, E. Ros, D. Bartres-Faz, Decreased Default Mode Network connectivity correlates with age-associated structural and cognitive changes, Frontiers in aging neuroscience 6 (2014) 256.

88. J.R. Andrews-Hanna, A.Z. Snyder, J.L. Vincent, C. Lustig, D. Head, M.E. Raichle, R.L. Buckner, Disruption of large-scale brain systems in advanced aging, Neuron 56(5) (2007) 924-35.

89. J.S. Damoiseaux, C.F. Beckmann, E.J. Arigita, F. Barkhof, P. Scheltens, C.J. Stam, S.M. Smith, S.A. Rombouts, Reduced resting-state brain activity in the "default network" in normal aging, Cerebral cortex (New York, N.Y. : 1991) 18(8) (2008) 1856-64.

90. P.J. Toussaint, S. Maiz, D. Coynel, J. Doyon, A. Messe, L.C. de Souza, M. Sarazin, V. Perlbarg, M.O. Habert, H. Benali, Characteristics of the default mode functional connectivity in normal ageing and Alzheimer's disease using resting state fMRI with a combined approach of entropy-based and graph theoretical measurements, Neurolmage 101 (2014) 778-86.

91. M.L. Balthazar, B.M. de Campos, A.R. Franco, B.P. Damasceno, F. Cendes, Whole cortical and default mode network mean functional connectivity as potential biomarkers for mild Alzheimer's disease, Psychiatry research 221(1) (2014) 37-42.

92. J. Zhou, M.D. Greicius, E.D. Gennatas, M.E. Growdon, J.Y. Jang, G.D. Rabinovici, J.H. Kramer, M. Weiner, B.L. Miller, W.W. Seeley, Divergent network connectivity changes in behavioural variant frontotemporal dementia and Alzheimer's disease, Brain : a journal of neurology 133(Pt 5) (2010) 1352-67.

93. R.C. Wolf, F. Sambataro, N. Vasic, N.D. Wolf, P.A. Thomann, C. Saft, G.B. Landwehrmeyer, M. Orth, Default-mode network changes in preclinical Huntington's disease, Experimental neurology 237(1) (2012) 191-8. 
94. A. Tessitore, F. Esposito, C. Vitale, G. Santangelo, M. Amboni, A. Russo, D. Corbo, G. Cirillo, P. Barone, G. Tedeschi, Default-mode network connectivity in cognitively unimpaired patients with Parkinson disease, Neurology 79(23) (2012) 2226-32.

95. N. Yao, R. Shek-Kwan Chang, C. Cheung, S. Pang, K.K. Lau, J. Suckling, J.B. Rowe, K. Yu, H. Ka-Fung Mak, S.E. Chua, S.L. Ho, G.M. McAlonan, The default mode network is disrupted in Parkinson's disease with visual hallucinations, Human brain mapping 35(11) (2014) 5658-66.

96. E.A. Disbrow, O. Carmichael, J. He, K.E. Lanni, E.M. Dressler, L. Zhang, N. Malhado-Chang, K.A. Sigvardt, Resting state functional connectivity is associated with cognitive dysfunction in non-demented people with Parkinson's disease, Journal of Parkinson's disease 4(3) (2014) 453-65.

97. M. Tahmasian, S.B. Eickhoff, K. Giehl, F. Schwartz, D.M. Herz, A. Drzezga, T. van Eimeren, A.R. Laird, P.T. Fox, H. Khazaie, M. Zarei, C. Eggers, C.R. Eickhoff, Resting-state functional reorganization in Parkinson's disease: An activation likelihood estimation meta-analysis, Cortex; a journal devoted to the study of the nervous system and behavior 92 (2017) 119-138.

98. B.D. Berman, J. Smucny, K.P. Wylie, E. Shelton, E. Kronberg, M. Leehey, J.R. Tregellas, Levodopa modulates small-world architecture of functional brain networks in Parkinson's disease, Movement disorders : official journal of the Movement Disorder Society 31(11) (2016) 1676-1684.

99. R.L. Folmer, J.J. Vachhani, S.M. Theodoroff, R. Ellinger, A. Riggins, Auditory Processing Abilities of Parkinson's Disease Patients, BioMed research international 2017 (2017) 2618587.

100. M. Nojszewska, B. Pilczuk, B. Zakrzewska-Pniewska, K. Rowinska-Marcinska, The auditory system involvement in Parkinson disease: electrophysiological and neuropsychological correlations, Journal of clinical neurophysiology : official publication of the American Electroencephalographic Society 26(6) (2009) 430-7.

101. C.J. Hardy, C.R. Marshall, H.L. Golden, C.N. Clark, C.J. Mummery, T.D. Griffiths, D.E. Bamiou, J.D. Warren, Hearing and dementia, Journal of neurology 263(11) (2016) 2339-2354.

102. S.J.G. Lewis, A. Dove, T.W. Robbins, R.A. Barker, A.M. Owen, Cognitive impairments in early Parkinson's disease are accompanied by reductions in activity in frontostriatal neural circuitry, Journal of Neuroscience 23(15) (2003) 6351-6356.

103. S.P. Caminiti, C. Siri, L. Guidi, A. Antonini, D. Perani, The neural correlates of spatial and object working memory in elderly and Parkinson's disease subjects, Behavioural neurology 2015 (2015) 123636.

104. S. Japee, K. Holiday, M.D. Satyshur, I. Mukai, L.G. Ungerleider, A role of right middle frontal gyrus in reorienting of attention: a case study, Frontiers in systems neuroscience 9 (2015) 23.

105. P. Manza, S. Zhang, C.S. Li, H.C. Leung, Resting-state functional connectivity of the striatum in early-stage Parkinson's disease: Cognitive decline and motor symptomatology, Human brain mapping $37(2)(2016) 648-62$.

106. P.A. Reuter-Lorenz, K.A. Cappell, Neurocognitive Aging and the Compensation Hypothesis, Current Directions in Psychological Science 17(3) (2008) 177-182.

107. M. Samuel, A.O. Ceballos-Baumann, J. Blin, T. Uema, H. Boecker, R.E. Passingham, D.J. Brooks, Evidence for lateral premotor and parietal overactivity in Parkinson's disease during sequential and bimanual movements. A PET study, Brain : a journal of neurology 120 ( Pt 6) (1997) 963-76. 
108. A. Dagher, A.M. Owen, H. Boecker, D.J. Brooks, The role of the striatum and hippocampus in planning: a PET activation study in Parkinson's disease, Brain : a journal of neurology 124(Pt 5) (2001) 1020-32.

109. K.L. Poston, S. YorkWilliams, K. Zhang, W. Cai, D. Everling, F.M. Tayim, S. Llanes, V. Menon, Compensatory neural mechanisms in cognitively unimpaired Parkinson disease, Annals of neurology 79(3) (2016) 448-63.

110. I. Rektorova, Resting-state networks in Alzheimer's disease and Parkinson's disease, Neuro-degenerative diseases 13(2-3) (2014) 186-8.

111. R. Lopes, C. Delmaire, L. Defebvre, A.J. Moonen, A.A. Duits, P. Hofman, A.F. Leentjens, K. Dujardin, Cognitive phenotypes in parkinson's disease differ in terms of brain-network organization and connectivity, Human brain mapping 38(3) (2017) 1604-1621.

112. G.W. Duncan, M.J. Firbank, J.T. O’Brien, D.J. Burn, Magnetic resonance imaging: a biomarker for cognitive impairment in Parkinson's disease?, Movement disorders : official journal of the Movement Disorder Society 28(4) (2013) 425-38.

113. W. Marks, L. Evers, M. Faber, M. Verbeek, N. De Vries, B. Bloem, Study design for a multi-modal approach to understanding parkinson's disease: The personalized parkinson project, Movement Disorders 32 (Supplement 2) (2017) 948.

114. Y. Chen, W. Yang, J. Long, Y. Zhang, J. Feng, Y. Li, B. Huang, Discriminative analysis of Parkinson's disease based on whole-brain functional connectivity, PloS one 10(4) (2015) e0124153.

115. A. Vo, W. Sako, K. Fujita, S. Peng, P.J. Mattis, F.M. Skidmore, Y. Ma, A.M. Ulug, D. Eidelberg, Parkinson's disease-related network topographies characterized with resting state functional MRI, Human brain mapping 38(2) (2017) 617-630.

116. Y. Zeighami, M. Ulla, Y. Iturria-Medina, M. Dadar, Y. Zhang, K.M. Larcher, V. Fonov, A.C. Evans, D.L. Collins, A. Dagher, Network structure of brain atrophy in de novo Parkinson's disease, Elife 4 (2015).

117. D. Long, J. Wang, M. Xuan, Q. Gu, X. Xu, D. Kong, M. Zhang, Automatic classification of early Parkinson's disease with multi-modal MR imaging, PloS one 7(11) (2012) e47714.

118. G. Kalpouzos, J. Persson, L. Nyberg, Local brain atrophy accounts for functional activity differences in normal aging, Neurobiology of aging 33(3) (2012) 623 e1-623 e13.

119. V. Rajagopalan, G.H. Yue, E.P. Pioro, Do preprocessing algorithms and statistical models influence voxel-based morphometry (VBM) results in amyotrophic lateral sclerosis patients? A systematic comparison of popular VBM analytical methods, Journal of magnetic resonance imaging : JMRI 40(3) (2014) 662-7.

120. E. Zhurakovskaya, J. Paasonen, A. Shatillo, A. Lipponen, R. Salo, R. Aliev, H. Tanila, O. Grohn, Global Functional Connectivity Differences between Sleep-Like States in Urethane Anesthetized Rats Measured by fMRI, PloS one 11(5) (2016) e0155343.

121. L.C. Dang, J.P. O'Neil, W.J. Jagust, Dopamine supports coupling of attention-related networks, The Journal of neuroscience : the official journal of the Society for Neuroscience 32(28) (2012) 9582-7. 
122. L. Krajcovicova, M. Mikl, R. Marecek, I. Rektorova, The default mode network integrity in patients with Parkinson's disease is levodopa equivalent dose-dependent, Journal of neural transmission (Vienna, Austria : 1996) 119(4) (2012) 443-54.

123. P. Delaveau, P. Salgado-Pineda, P. Fossati, T. Witjas, J.P. Azulay, O. Blin, Dopaminergic modulation of the default mode network in Parkinson's disease, European neuropsychopharmacology : the journal of the European College of Neuropsychopharmacology 20(11) (2010) 784-92.

124. I. Litvan, D. Aarsland, C.H. Adler, J.G. Goldman, J. Kulisevsky, B. Mollenhauer, M.C. Rodriguez-Oroz, A.I. Troster, D. Weintraub, MDS Task Force on mild cognitive impairment in Parkinson's disease: critical review of PD-MCI, Movement disorders : official journal of the Movement Disorder Society 26(10) (2011) 1814-24.

125. I. Litvan, J.G. Goldman, A.I. Troster, B.A. Schmand, D. Weintraub, R.C. Petersen, B. Mollenhauer, C.H. Adler, K. Marder, C.H. Williams-Gray, D. Aarsland, J. Kulisevsky, M.C. Rodriguez-Oroz, D.J. Burn, R.A. Barker, M. Emre, Diagnostic criteria for mild cognitive impairment in Parkinson's disease: Movement Disorder Society Task Force guidelines, Movement disorders : official journal of the Movement Disorder Society 27(3) (2012) 349-56.

126. M. Emre, D. Aarsland, R. Brown, D.J. Burn, C. Duyckaerts, Y. Mizuno, G.A. Broe, J. Cummings, D.W. Dickson, S. Gauthier, J. Goldman, C. Goetz, A. Korczyn, A. Lees, R. Levy, I. Litvan, I. McKeith, W. Olanow, W. Poewe, N. Quinn, C. Sampaio, E. Tolosa, B. Dubois, Clinical diagnostic criteria for dementia associated with Parkinson's disease, Movement disorders : official journal of the Movement Disorder Society 22(12) (2007) 1689-707; quiz 1837.

127. B. Dubois, D. Burn, C. Goetz, D. Aarsland, R.G. Brown, G.A. Broe, D. Dickson, C. Duyckaerts, J. Cummings, S. Gauthier, A. Korczyn, A. Lees, R. Levy, I. Litvan, Y. Mizuno, I.G. McKeith, C.W. Olanow, W. Poewe, C. Sampaio, E. Tolosa, M. Emre, Diagnostic procedures for Parkinson's disease dementia: recommendations from the movement disorder society task force, Movement disorders : official journal of the Movement Disorder Society 22(16) (2007) 2314-24.

128. K. Dujardin, A.J. Moonen, H. Behal, L. Defebvre, A. Duhamel, A.A. Duits, L. Plomhause, C. Tard, A.F. Leentjens, Cognitive disorders in Parkinson's disease: Confirmation of a spectrum of severity, Parkinsonism \& related disorders 21(11) (2015) 1299-305. 
RESTING-STATE FMRI IN PARKINSON'S DISEASE PATIENTS WITH COGNITIVE IMPAIRMENT 55 

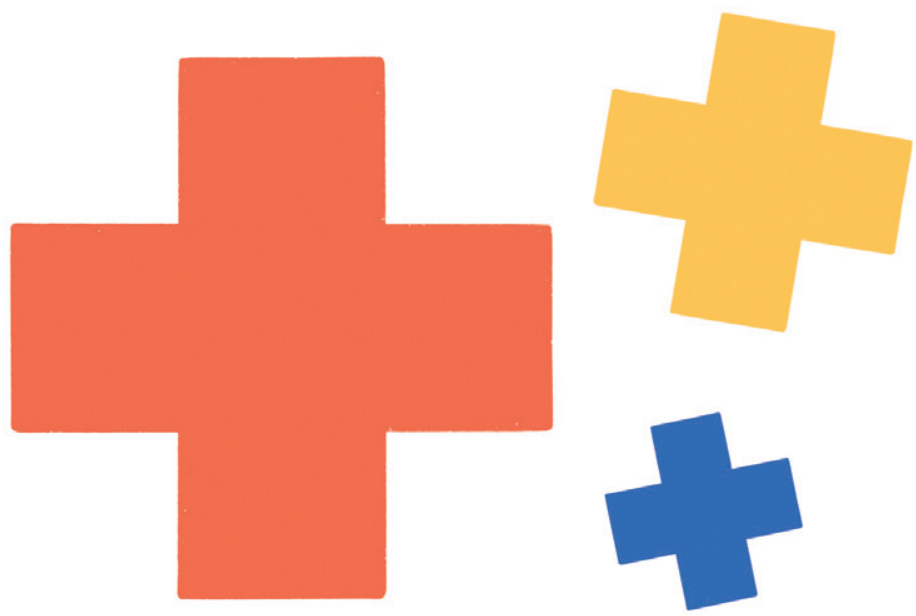


\section{CHAPTER 3}

\section{Promising non-}

pharmacological therapies in PD: Targeting late stage disease and the role of computer based cognitive training 


\section{ABSTRACT}

Non-pharmacological interventions are increasingly being acknowledged as valuable treatment options to overcome or reduce functional problems in patients with Parkinson's disease (PD). There is a wide range of such non-pharmacological treatments for which the supportive evidence is emerging. Physiotherapy is one good example in this domain. However, there are also several promising non-pharmacological treatment strategies that have thus far received less research attention. Here, we describe two relatively new, but encouraging approaches. First, we focus on a hitherto largely overseen subgroup of PD, namely those with late-stage disease, a population that is often excluded from clinical studies. Importantly, the aims and therapeutic strategies in late-stage PD differ considerably from those in early-stage $\mathrm{PD}$, and an emphasis on non-pharmacological management is particularly important for this vulnerable subgroup. Second, we focus on computer-based cognitive training, as an example of a relatively new intervention that includes innovative elements such as personalized training, artificial intelligence, and virtual reality. We review the latest evidence, practical considerations and future research perspectives, both for non-pharmacological approaches in late-stage PD and for computer-based cognitive training. 


\section{BACKGROUND}

Despite the clear beneficial effects of pharmacological and surgical treatments, patients with Parkinson's disease (PD) continue to experience a wide range of motor and non-motor symptoms that lead to marked functional impairment, disability and reduced quality of life. Non-pharmacological interventions are increasingly being recognized as valuable treatment options to help overcome or reduce these functional problems [1]. A wide range of nonpharmacological treatment options is now available (Table 1 ). In general, the supportive scientific evidence for these treatments is increasing [1], particularly for interventions related to physiotherapy and exercise. For example, there is strong evidence that cueing strategies are effective to overcome episodes of freezing of gait [2], and that exercise leads to positive effects on both motor and non-motor symptoms (such as depression) [3]. New rehabilitation approaches, e.g. using virtual reality [4] or dedicated dual task training [5], are also emerging. Furthermore, alternative types of exercise, like dance or t'ai chi, are gaining popularity, both among patients and the scientific community. However, several other non-pharmacological interventions look promising at first sight, but have not been studied extensively. Here, we focus on two such non-pharmacological treatments. First, we will focus on a hitherto largely overseen subgroup of PD, namely those with late-stage disease (defined as Hoehn and Yahr (HY) stage 4-5), a population that is often excluded from clinical studies. Non-pharmacological management is particularly important for this vulnerable subgroup, because many symptoms no longer respond adequately to pharmacological treatment [6]. Yet, most research has thus far focused largely on PD patients in HY stages 1e3. Second, we will focus on computer-based cognitive training (CT) and cognitive gaming, which are relatively new non-pharmacological interventions. We will not offer a detailed systematic overview, but rather share the overall evidence on the effectiveness for both interventions, and offer insights into the potential practical implications and objectives for future research. 
Table 1. Non-pharmacological interventions in PD. These are examples are not exhaustive, but merely illustrate the wide range of therapeutic options in this arena.

Intervention*

\begin{tabular}{ll}
\hline Cognitive training & Cognitive training, computer-based cognitive training, cognitive gaming \\
\hline Diet & $\begin{array}{l}\text { Personalized treatment aiming to improve nutritional status (e.g. avoiding } \\
\text { malnutrition, managing protein intake relative to levodopa intake) }\end{array}$ \\
\hline Exercise & Aerobic exercise, dancing, tai chi, strength training \\
\hline $\begin{array}{l}\text { Non-invasive brain } \\
\text { stimulation }\end{array}$ & Transcranial magnetic stimulation, transcranial direct current stimulation \\
\hline
\end{tabular}

Occupational therapy Personalized treatment aiming to enable patients to engage in meaningful roles and activities and to support self-management

\begin{tabular}{ll}
\hline Physiotherapy & $\begin{array}{l}\text { Personalized treatment aiming to maximize movement quality, functional } \\
\text { independence and general fitness; minimizing secondary complications; } \\
\text { optimizing safety; supporting self-management and participation. }\end{array}$ \\
\hline
\end{tabular}

$\begin{array}{ll}\begin{array}{l}\text { Speech and language } \\ \text { therapy }\end{array} & \begin{array}{l}\text { Personalized treatment aiming to improve communication, language } \\ \text { therapy, swallowing training }\end{array}\end{array}$

Complementary Wide range, including music therapy, mindfulness training, yoga

interventions

*In alphabetical order.

\section{NON-PHARMACOLOGICAL TREATMENT OPTIONS IN LATE-STAGE PD}

\section{LATEST EVIDENCE}

Although patients with late-stage PD would hypothetically benefit from non-pharmacological interventions, surprisingly, the available studies suggest that non-pharmacological strategies are less effective in late-stage PD compared to earlier disease stages. A recent non-controlled and non-randomized trial on multidisciplinary treatment (performed in an inpatient setting) showed a small improvement on the Functional Independence Measures, a scale that measures activities of daily living [7]. This study included non-demented PD-patients in HY-stage 3-5. A sub-analysis showed improvements in $\mathrm{HY}$-stage 3-4, but not in HY-stage 5. Also, two trials on home-based exercise and strategy training showed no improvement in fall rates in H\&Y stage 4 , while less falls were reported in earlier disease stages [8,9]. A possible explanation for this limited effectiveness in late stage PD is that these patients often have cognitive impairment, while improvements in motor function depend in part on cognitive performance (e.g. to learn new movement strategies, or comply with instructions) [10]. Another explanation may be the complexity of the intervention; the effectiveness is potentially compromised by low adherence to these complex interventions. 


\section{PRACTICAL IMPLICATIONS}

The absent effect of non-pharmacological interventions in late-stage PD suggests that effective interventions in early disease stages cannot be translated directly to late-stage PD. Indeed, therapeutic strategies in late-stage PD differ from treatments in other disease stages in several ways, and clinical trials should take these differences into account. First, as patients become more dependent on caregivers, the treatment approach shifts from the traditional patient-therapist dyad, to addressing the needs of patient and the care system. Educating caregivers about PD may lead to improvements in disease symptoms, mood and fatigue [11], but this awaits confirmation in well-designed trials. Therapy in later stages should therefore not only focus on training the patient, but also on instructing and educating informal and professional caregivers.

Second, the cognitive capacities of the patient and cognitive demands of the therapy must be taken into account. One study compared three types of patient instructions for speech and language therapy: explicit, visual analogy, and verbal analogy [12]. Visual analogy was superior to explicit instruction, while verbal analogy was cognitively least demanding, yielding the best result. Hence, future research should investigate the cognitive demands of therapies and identify the most feasible approaches.

Importantly, treatment goals differ in late-stage PD, and outcome measures in clinical trials should accommodate this. As patients become dependent, treatment goals shift from improvement of function to maintaining function and preventing complications [13]. Potential complications that can be addressed by non-pharmacological therapies are pressure ulcers, malnutrition, dehydration, aspiration pneumonia and falls. In order to prevent pressure ulcers, consensus-based guidelines recommend involvement of physiotherapy, occupational therapy and dietary treatment $[14,17]$. The physiotherapist and occupational therapist can evaluate and optimize posture when patients are bedridden or wheelchair bound. The dietitian can advise on protein-intake, as protein is implicated in wound healing. However, no studies have specifically evaluated these therapies in PD. Malnutrition, dehydration and aspiration pneumonia are associated with dysphagia, which can be addressed by speech language therapy and dietary treatment $[16,17]$.

Finally, treatment strategies should be adapted to the specific needs and capabilities of latestage PD patients, e.g. by using less cognitively demanding strategies or strategies that better stimulate adherence. Recent work focused on integrating non-pharmacological strategies into a palliative care approach [18]. Palliative care focuses on supporting patients and families, and on anticipating and planning future care to prevent and reduce disease burden and suffering. Functional decline is not considered as treatment failure, but as natural disease course. Allied health professionals can contribute to palliative care by treating in a holistic framework and helping patients to maintain meaningful activities [19]. For example, the speech and language therapist integrates information on the patient's favorite culinary dish in the treatment. Or the physiotherapist takes on a counseling role discussing grief after patients lose the ability 
to walk. The most important barriers include a lack of timely referral and inadequate funding for treatment. Also, personal barriers, such as a lack of knowledge, insufficient experience and lack of inter-professional collaboration may be important.

\section{FUTURE RESEARCH}

Several major research projects are ongoing. In the UK, the OPTCARE Neuro trial evaluates the short-term effectiveness of an intervention by a multidisciplinary palliative care team in long-term neurological conditions including PD. The Care of Late Stage Parkinsonismstudy (CLaSP-study) is a multicenter cohort study in six European countries that evaluates the healthcare needs and provision of care to patients in late-stage disease. These studies are expected to report their results shortly, informing us on the best approaches of treating late-stage PD. Future research should also focus on identifying best practices and finding evidence for non-pharmacological treatment in late-stage disease. However, studying a highly-disabled patient population that is often institutionalized and experiences cognitive decline, is challenging for a number of reasons. First, recruitment and enrollment of patients can be difficult as some patients have lost contact with their treating medical specialist, and are unable to visit outpatient clinics. Also, informed consent can be harder to obtain as it often requires proxy consent. Second, retaining patients during the study is more complex because additional strain (caused by participating in research) is not tolerated easily. Also, a high drop-out rate should be anticipated due to disease progression, moving to institutions, or death. Third, conventional outcomes, like the MDS-UPDRS, are not validated for this specific population. Lastly, compliance can be challenging when multiple caregivers are involved.

\section{COGNITIVE TRAINING}

\section{LATEST EVIDENCE}

Patients with PD are at risk of cognitive decline in both early and late disease stages. Cognitive deficits generally seen in PD include impairment in cognitive flexibility, planning, reasoning, attention, and verbal processes [20]. CT can be a potentially effective treatment strategy where specific cognitive tasks are offered to train different cognitive domains. In PD, the evidence on CT is promising. In-person (non-computer-based) CT can improve, among others, executive function, memory and processing speed. For example, a 4-week executive function training $(n=12)$ in the hospital resulted in improved executive function and working memory, as compared to a control group $(n=14)$ receiving occupational therapy, physiotherapy and physical treatment [21]. Another study showed that 13 weeks of in-person CT ( $n=22$, training attention, language, memory, verbal processing, executive function, theory of mind, general cognition, and functional disability) led to improved processing speed, visual memory, functional disability, and theory of mind, as compared to a control group $(n=22)$ receiving occupational training [22]. 
Recent technological innovations make it possible to create flexible computer-based trainings, adding benefits over in-person CT in terms of cost-effectiveness, scalability, flexibility, and constant adaptability of the training content. Evidence on the effectiveness of computer-based $\mathrm{CT}$ is emerging. Edwards et al. found improved speed of processing and field of view after 20 h of processing speed training over three months (training processing of visual stimuli, visual working memory and selective attention) ( $n=44)$, as compared to a waiting list control group $(n=43)$ [23]. Another study compared intensive individualized CT $(n=12)$ combining computerbased and in-person training to speech therapy $(n=12)$ [24]. After $9 \mathrm{~h}$ of training, the CT group performed better on various cognitive measures, including attention, information processing speed, memory, visuospatial and visuo-constructive abilities, semantic and verbal fluency, and executive function. Also, improved learning and memory retention was found after 14 2-h sessions of a combined in-person psychoeducational intervention and an individualized computer-based CT program $(n=35)$ [25]. In a recent review, CT was found to modestly but significantly improve overall cognition, working memory, processing speed, and executive functions. However, no significant effects were found on the cognitive domains memory and visuo-spatial skills [26]. Larger RCTs and clearly-defined trainings clearly remain required.

\section{PRACTICAL IMPLICATIONS}

Whereas $C T$ includes repeating cognitive tasks to improve cognition, the intervention can be made more engaging by including gaming features to transform a generally monotonous task into an enjoyable experience, which may positively influence motivation and adherence [27]. Cognitive games are expected to provide additional benefits to $\mathrm{CT}$, since they incorporate positive feedback, are gratifying, and can be tailored to the individuals' capacities. The effectiveness of pure cognitive games (with solely a cognitive rationale) has not yet been reported in PD, but new studies are in progress [28]. Moreover, exergames (i.e., video games that incorporate a form of exercise) can not only reduce the severity of motor symptoms; PD patients also seem to enjoy the process [29]. Additionally, exergames likely require cognitive abilities, and may therefore even improve both physical and cognitive functioning [29].

One great advantage of gaming is that games can be used to simultaneously asses and train cognitive domains, meaning that training can be readily adjusted to the players' individual cognitive capacities in terms of content and difficulty (Fig. 1). The adaptation of cognitive games can be enhanced further by Artificial Intelligence (AI) as an automated adaptation tool. The gaming system automatically and in real-time responds to the interaction of the user with the game, mapping those interactions to levels of cognitive states and personalizing the game accordingly, theoretically resulting in more efficient therapies. In addition to the adaptability of gaming systems using Al, virtual reality (VR) will probably also play a role in $\mathrm{CT}$, since realistic 3D environments can be tailored to the patients' needs. VR is found to improve patients' motivation, and VR experiences are more likely to be generalized or transferred outside of the training situation [30]. An overview of aspects that must be considered when designing a computer-based intervention for patients with PD are summarized in Table 2. 


\section{FUTURE RESEARCH}

Current research on computer-based CT has some limitations that should be considered in future studies. For example, hardly any training details are presented, limiting the conclusions that can be drawn in terms of transfer (e.g. what tasks are responsible for what cognitive effects). Future studies should also include larger sample sizes, use adequate control groups, longitudinal designs, a standardized neuropsychological test battery and explicitly define cognitive inclusion criteria. Additionally, to see whether cognitive games provide an additional benefit over (computer-based) CT in PD, studies need to explicitly examine the role of gamification on training adherence. Despite current limitations, computer-based CT is a promising method to train cognitive functions in patients with PD [26].

\section{CONCLUSION}

Here, we have described two relatively new, but encouraging approaches in the nonpharmacological treatment of patients with PD. More research is needed to further develop and study the optimal strategies for patients with PD in different disease stages. Considering computer-based CT and cognitive gaming, many advancements are expected, both in terms of technological developments and quality of scientific studies. This may also be relevant to patients with late-stage PD, who should be well-represented in future studies on nonpharmacological treatments in PD in general (and computer-based CT in particular), thereby increasing the treatment repertoire for the total population of patients with PD.

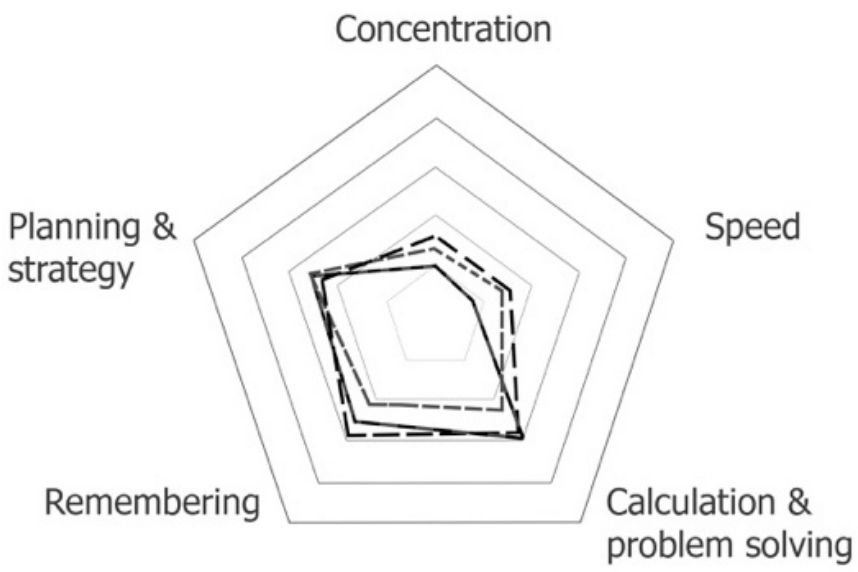

\section{Baseline $\quad$ First follow-up $\quad$ Second follow-up}

Figure 1. Cognitive scores on separate cognitive domains over time, which can be used to personalize the training. In the example of a particular patient shown here, the results of a baseline assessment indicate declined concentration and slower speed. These domains were subsequently trained. At first follow-up, modest improvements were found in these domains. When additional training was provided, further improved scores were noted at the second follow-up assessment. 
Table 2. Recommendations for the design of a cognitive game for patients with PD (ranked per category).

\begin{tabular}{lll} 
User interface and experience & Adaptability and feedback & Motivational aspects \\
\hline $\begin{array}{l}\text { Comprehendible and simple UI } \\
\text { design }\end{array}$ & $\begin{array}{l}\text { Adopt levels of increasing } \\
\text { complexity }\end{array}$ & Clear instructions and goals \\
\hline Limit the need for double clicking & $\begin{array}{l}\text { Dynamic difficulty adaptation } \\
\text { mechanism }\end{array}$ & Implement social functions \\
\hline $\begin{array}{l}\text { Limit the need for moving the } \\
\text { cursor }\end{array}$ & $\begin{array}{l}\text { Encouraging, positive feedback } \\
\text { mechanism }\end{array}$ & Multiplayer compatibility \\
\hline Limit keyboard use & Immediate feedback response & Competitive elements \\
\hline Limit simultaneous key & & Easy setup \\
combinations & & Cross-platform compatibility \\
\hline Make font sizes adjustable & & \\
\hline Make colour contrast adjustable & &
\end{tabular}

Ignore accidental longer touch durations

\section{CONFLICT OF INTEREST}

The authors declare no conflict of interest.

\section{ACKNOWLEDGEMENT}

Professor Bastiaan R. Bloem was supported by a centre grant of the National Parkinson Foundation (NPF). Dr. Nienke M. de Vries was supported by a research grant of The Netherlands Organisation for Health Research and Development (525001008). 


\section{REFERENCES}

1. B.R. Bloem, N.M. de Vries, G. Ebersbach, Nonpharmacological treatments for patients with Parkinson's disease, Mov. Disord. 30 (11) (2015) 1504e1520.

2. J. Nonnekes, A.H. Snijders, J.G. Nutt, G. Deuschl, N. Giladi, B.R. Bloem, Freezing of gait: a practical approach to management, Lancet Neurol. 14 (7) (2015) $768 \mathrm{e} 778$.

3. M.E. Cusso, K.J. Donald, T.K. Khoo, The impact of physical activity on non-motor symptoms in Parkinson's disease: a systematic review, Front. Med. (Lausanne) 3 (2016) 35.

4. A. Mirelman, L. Rochester, I. Maidan, S. Del Din, L. Alcock, F. Nieuwhof, M.O. Rikkert, B.R. Bloem, E. Pelosin, L. Avanzino, G. Abbruzzese, K. Dockx, E. Bekkers, N. Giladi, A. Nieuwboer, J.M. Hausdorff, Addition of a nonimmersive virtual reality component to treadmill training to reduce fall risk in older adults (V-TIME): a randomised controlled trial, Lancet 388 (10050) (2016) 1170e1182.

5. C. Strouwen, E. Molenaar, L. Munks, S.H.J. Keus, J.C.M. Zijlmans, W. Vandenberghe, B.R. Bloem, A. Nieuwboer, Training dual tasks together or apart in Parkinson's disease: results from the duality trial, Mov. Disord. 32 (8) (2017) 1201e1210.

6. M. Coelho, J.J. Ferreira, Late-stage Parkinson disease, Nat. Rev. Neuro 8 (8) (2012) 435e442.

7. Y. Kaseda, J. Ikeda, K. Sugihara, T. Yamawaki, T. Kohriyama, M. Matsumoto, Therapeutic effects of intensive inpatient rehabilitation in advanced Parkinson's disease, Neurol. Clin. Neurosci. 5 (1) (2017) 18 e21.

8. A. Ashburn, L. Fazakarley, C. Ballinger, R. Pickering, L.D. McLellan, C. Fitton, A randomised controlled trial of a home based exercise programme to reduce the risk of falling among people with Parkinson's disease, J. Neurol. Neurosurg. Psychiatry 78 (7) (2007) 678e684.

9. C.G. Canning, C. Sherrington, S.R. Lord, J.C. Close, S. Heritier, G.Z. Heller, K. Howard, N.E. Allen, M.D. Latt, S.M. Murray, S.D. O'Rourke, S.S. Paul, J. Song, V.S. Fung, Exercise for falls prevention in Parkinson disease: a randomized controlled trial, Neurology 84 (3) (2015) 304e312.

10. D. Ferrazzoli, P. Ortelli, R. Maestri, R. Bera, R. Gargantini, G. Palamara, M. Zarucchi, N. Giladi, G. Frazzitta, Focused and sustained attention is modified by a goal-based rehabilitation in parkinsonian patients, Front. Behav. Neurosci. 11 (2017) 56.

11. M. Makoutonina, R. lansek, P. Simpson, Optimizing care of residents with Parkinsonism in supervised facilities, Park. Relat. Disord. 16 (5) (2010) $351 \mathrm{e} 355$.

12. C.Y. Tse, A. Wong, T. Whitehill, E. Ma, R. Masters, Examining the cognitive demands of analogy instructions compared to explicit instructions, Int. J. Speech Lang. Pathol. 18 (5) (2016) 465e472.

13. D.L. Radder, I.H. Sturkenboom, M. van Nimwegen, S.H. Keus, B.R. Bloem, N.M. de Vries, Physical therapy and occupational therapy in Parkinson's disease, Int. J. Neurosci. (2017) 1e14.

14. S.H.Keus,M.Munneke,M.Graziano,etal.,EuropeanPhysiotherapyGuideline for Parkinson's Disease KNGF, ParkinsonNet, The Netherlands, 2014. 
15. I. Sturkenboom, M. Thijssen, J. Gons-Van Elsacker, I. Jansen, A. Maasdam, M. Schulten, D. Vijver-Visser, E.J.M. Steultjens, B.R. Bloem, M. Munneke, Ergotherapie bij de ziekte van Parkinson, een richtlijn van Ergotherapie Nederland, Uitgeverij LEMMA, Den Haag, 2008.

16. H. Kalf, B. De Swart, M. Bonnier-Baars, J. Kanters, M. Hofman, J. Kocken, M. Miltenburg, B.R. Bloem, M. Munneke, Logopedie bij de ziekte van Parkinson: een richtlijn van de Nederlandse Vereniging voor Logopedie en Foniatrie, Uitgeverij LEMMA, Den Haag, 2008.

17. M.J.M.D. Van Asseldonk, H.C. Dicke, B.J.W. Van den Beemt, D.J. Van den Berg, S. Ter Borg, G.M. Duin, S.L.H.M. Govers, J.J. Van Teeffelen, J.I. Hoff, B. Van Harten, B.R. Bloem, S.H.J. Keus, M. Munneke, Richtlijn Dietetiek bij de ziekte van Parkinson, Boom Lemma Uitgevers, Den Haag, 2012.

18. R.Bouca-Machado,N.Titova,K.R.Chaudhuri,B.R.Bloem,J.J.Ferreira,Palliative care for patients and families with Parkinson's disease, Int. Rev. Neurobiol. 132 (2017) 475e509.

19. M. Waldron, W. Kernohan, F. Hasson, S. Foster, B. Cochrane, C. Payne, Allied health professionals's views on palliative care for people with advanced Parkinson's disease, Int. J. Ther. Rehabil. 18 (1) (2011) 48 e58.

20. K. Pigott, J. Rick, S.X. Xie, H. Hurtig, A. Chen-Plotkin, J.E. Duda, J.F. Morley, L.M. Chahine, N. Dahodwala, R.S. Akhtar, A. Siderowf, J.Q. Trojanowski, D. Weintraub, Longitudinal study of normal cognition in Parkinson disease, Neurology 85 (15) (2015) $1276 \mathrm{e} 1282$.

21. G. Sammer, I. Reuter, K. Hullmann, M. Kaps, D. Vaitl, Training of executive functions in Parkinson's disease, J. Neurol. Sci. 248 (1e2) (2006) 115 e119.

22. Pena, N. Ibarretxe-Bilbao, I. Garcia-Gorostiaga, M.A. Gomez-Beldarrain, M. Diez-Cirarda, N. Ojeda, Improving functional disability and cognition in Parkinson disease: randomized controlled trial, Neurology 83 (23) (2014) $2167 e 2174$.

23. J.D. Edwards, R.A. Hauser, M.L. O'Connor, E.G. Valdes, T.A. Zesiewicz, E.Y. Uc, Randomized trial of cognitive speed of processing training in Parkinson disease, Neurology 81 (15) (2013) 1284e1290.

24. A.P. Paris, H.G. Saleta, M. de la Cruz Crespo Maraver, E. Silvestre, M.G. Freixa, C.P. Torrellas, S.A. Pont, M.F. Nadal, S.A. Garcia, M.V. Bartolome, V.L. Fernandez, A.R. Bayes, Blind randomized controlled study of the efficacy of cognitive training in Parkinson's disease, Mov. Disord. 26 (7) (2011) $1251 e 1258$.

25. S.L. Naismith, L. Mowszowski, K. Diamond, S.J. Lewis, Improving memory in Parkinson's disease: a healthy brain ageing cognitive training program, Mov. Disord. 28 (8) (2013) 1097e1103.

26. I.H. Leung, C.C. Walton, H. Hallock, S.J. Lewis, M. Valenzuela, A. Lampit, Cognitive training in Parkinson disease: a systematic review and meta-analysis, Neurology 85 (21) (2015) $1843 e 1851$.

27. J. Lumsden, E.A. Edwards, N.S. Lawrence, D. Coyle, M.R. Munafo, Gamification of cognitive assessment and cognitive training: a systematic review of applications and efficacy, JMIR serious games 4 (2) (2016) e11.

28. S.C. van de Weijer, A.A. Duits, B.R. Bloem, R.P. Kessels, J.F. Jansen, S. Kohler, G. Tissingh, M.L. Kuijf, The Parkin'Play study: protocol of a phase II randomized controlled trial to assess the effects of a health game on cognition in Parkinson's disease, BMC Neurol. 16 (1) (2016) 209. 
29. G. Barry, B. Galna, L. Rochester, The role of exergaming in Parkinson's disease rehabilitation: a systematic review of the evidence, J. Neuroeng Rehabil. 11 (2014) 33.

30. E.B. Larson, M. Feigon, P. Gagliardo, A.Y. Dvorkin, Virtual reality and cognitive rehabilitation: a review of current outcome research, NeuroRehabilitation 34 (4) (2014) 759e772. 
PROMISING NON-PHARMACOLOGICAL THERAPIES IN PD 69 


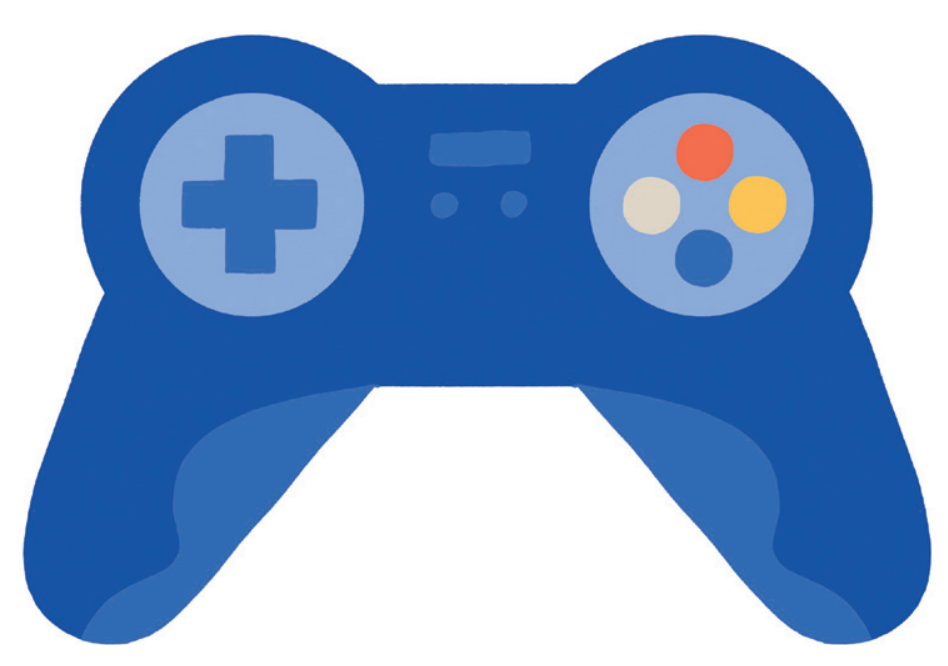




\section{CHAPTER 4}

\section{Do-It-Yourself Gamified Cognitive Training: A Viewpoint}




\section{ABSTRACT}

Background: Cognitive decline is an important nonmotor symptom in Parkinson's disease (PD). Unfortunately, very few treatment options are available. Recent research pointed to small positive effects of nonpharmacological cognitive training in PD. Most of these trainings are performed under supervision and solely computerized versions of (traditional) paper-pencil cognitive training programs, lacking rewarding gamification stimulants that could help to promote adherence.

Objective: By describing 3 different self-invented ways of cognitive gaming in patients with $\mathrm{PD}$, we aimed to raise awareness for the potential of gamified cognitive training in PD patients. In addition, we hoped to inspire the readers with our case descriptions, highlighting the importance of both personalization and cocreation in the development of games for health.

Results: In this viewpoint, we have presented 3 PD patients with different ages, with different disease stages, and from various backgrounds, who all used self-invented cognitive training, including elements of personalization and gamification. To indicate generalization into a larger PD population, the recruitment results from a recent cognitive game trial are added.

Discussion: The presented cases show similarities in terms of awareness of their cognitive decline and the ways this process could potentially be counteracted, by looking for tools to train their cognition. Based on the response of the recruitment procedure, there seems to be interest in gamified cognitive training in a larger PD population too. Gamification may add to traditional therapies in terms of personalization and adherence. Positive results have already been found with gamified trainings in other populations, and the cases described here suggest that PD is also an attractive area to develop and test gamified cognitive trainings. However, no results of gamified cognitive trainings in PD have been published to date. This suggests an unmet need in this area and may justify the development of gamified cognitive training and its evaluation, for which our considerations can be used. 


\section{INTRODUCTION}

\section{BACKGROUND}

Parkinson's disease (PD) is a neurodegenerative disorder characterized by both motor and nonmotor symptoms. Mild cognitive impairment can already be present in up to $40 \%$ of newly diagnosed PD patients [1] and more marked decline can ultimately be seen in up to $83 \%$ of patients [2]. Cognitive impairment is associated with a decreased quality of life, an increased caregiver burden, and an increased risk of developing dementia [3]. Unfortunately, very few treatment options are available. The only effective pharmacological treatment (rivastigmine) provides limited improvements in memory and language [4]. Recent research has pointed to small positive effects of nonpharmacological cognitive trainings on working memory, processing speed, and executive function [5-7], suggesting that these interventions could possibly attenuate cognitive deficits in PD. Many of the investigated cognitive trainings in PD are performed under supervision and include solely computerized versions of paper-pencil (traditional) cognitive trainings. These traditional cognitive trainings involve repetitive execution of cognitive tasks but lack gamification stimulants. Gamification of cognitive training can be used to promote adherence, such as reward and engagement, and could eventually improve health outcomes. Both personalization and gamification could increase the adherence to and effectiveness of cognitive training in PD. Although some previously investigated interventions adapted to the user performance, adherence variables were unfortunately insufficiently reported across these studies. Therefore, we are currently unable to conclude that gamification of cognitive training is indeed more attractive for PD patients and results in increased adherence rates. Hence, more research is needed in the area of gamified cognitive training. Before we can test the effectiveness of such gamified cognitive trainings, it would be helpful to explore whether PD patients are interested in using gamified cognitive trainings at all.

\section{OBJECTIVES}

In this viewpoint, we have presented 3 independent histories of PD patients with different ages, with different disease stages, and from various backgrounds, who all used self-invented cognitive training that included elements of personalization and gamification. Using computer videogames, card games, or real-life routines, these patients self-trained their cognitive abilities, which are essential for activities of daily living. We will discuss the training types and present the similarities and differences between these cases. We additionally report on recruitment data from a recent gamified cognitive training trial [8]. By describing 3 different self-invented ways of cognitive gaming in patients with PD, we aimed to raise awareness for the potential of gamified cognitive training in PD patients. Additionally, we hoped to inspire the readers with our case descriptions, highlighting the importance of both personalization and cocreation in the development of games for health. Finally, we have presented some considerations for future gamified cognitive training development and evaluation. 


\section{RESULTS}

\section{CASE 1}

This 64-year-old man with PD had a disease duration of 20 years and a Hoehn and Yahr Stage of III, indicating a mild-to-moderate bilateral disease and some postural instability but being physically independent (the range according to the Hoehn and Yahr stages is from 0 [no symptoms] to V [severely disabled and wheelchair bound]) [9]. In the course of his disease, he started experiencing postural instability, decreased memory performance, and depressive symptoms. His passion was virtual car racing, and he customized a computer videogame racing simulator (called iRacing, by iRacing.com Motorsport Simulations) with a trajectory on the Nürburgring Nordschleife circuit (Germany; see Figure 1 and Multimedia Appendix 1). At the time, he was treated with a levodopa equivalent daily dose of $1285 \mathrm{mg}$, including a daily dose of $3 \mathrm{mg}$ Ropinirole dopamine agonist. He started racing on a daily basis in his simulator and challenged himself to improve on every race lap. He assessed his performance by remembering the influence of variances in turns on lap times. A race simulator challenges various cognitive functions (attention, decision making, and memory) as well as motor functions (reaction times and perceptuomotor skills). In the following months, he experienced improved driving skills in real life and better attentional performance while driving a real car, outside of the simulator. The patient's spouse believed her partner had an extended attentional span after playing the game regularly. His compliance was excellent, as the pursuit of the perfect race lap on the circuit was an intrinsic motivation for creating a gamified cognitive training task. He feels that pushing the boundaries prevents a rapid cognitive decline, and he has now faithfully used his simulator for over 5 years.

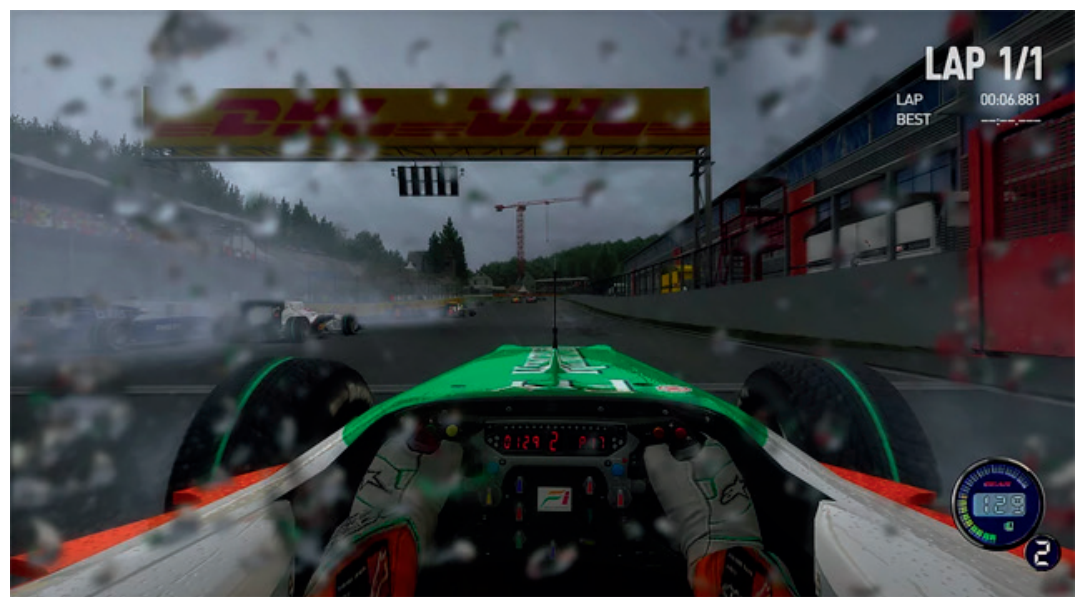

Figure 1. Screenshot of the racing simulator game played by Case 1 . 


\section{CASE 2}

The second case is a 67-year-old woman with PD in Hoehn and Yahr Stage II (bilateral involvement without impairment of balance) and a disease duration of 12 years. Soon after retiring as a financial consultant in the field of education, she became afraid that the decreased working load on her brain would result in memory loss. A few years into the disease, she indeed started experiencing memory loss, which motivated her to train herself in daily real-life situations. Specifically, she has developed several daily routines to train her memory. After waking up, she tries to remember all meetings for the upcoming day. She, afterward, checks her calendar to see if she was right. Also, if she is outdoors and plans a new meeting with a friend, she will note it in her calendar only by the time she comes home. Afterward, she will check to see if she remembered the correct date and time. Also, she manually enters frequently used phone numbers, even though she has saved them as contacts in her smartphone. Furthermore, when she plans on shopping for groceries, she makes a shopping list on paper that includes all the needed ingredients. In the store, however, she will not consult this list, but instead she will buy all products from memory. The shopping list is ultimately referred to as a checklist for completion. This type of real-life training requires multiple cognitive functions, including planning and memory. The patient feels that these self-invented routines keep her memory stable at an acceptable level. She is now confident that she is able to remember almost anything, and she has never heard from others that she forgot something. Importantly, compliance was again excellent, as she has been using these daily routines for over 5 years now.

\section{CASE 3}

The third case is a 68-year-old woman with PD in Hoehn and Yahr Stage III and a disease duration of 10 years. She has been living in South Africa volunteering as a community development worker for 28 years and has raised 5 children. After returning to the Netherlands, she was diagnosed with PD in 2009. In the following years, she started noticing cognitive problems, including concentration and memory deficits. She applied to a Dutch Web-based Bridge game service (called StepBridge, by StepBridge Foundation, see Figure 2), where she could play Bridge against gamers of similar difficulty levels at any time this would fit her schedule. This Bridge game requires several cognitive functions, including attention, reasoning, decision making, and memory. She reports subjective benefits in terms of both concentration and memory, which is also observed by her spouse. Compliance was again outstanding, as she has been playing StepBridge regularly for almost 10 years now. 


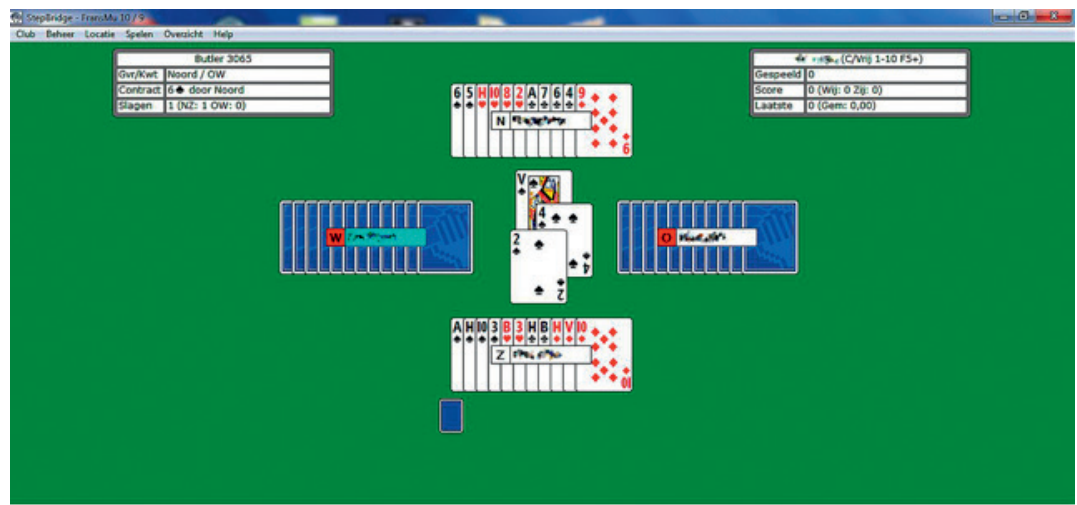

Figure 2. Screenshot of the StepBridge game played by Case 3.

\section{GENERALIZATION OF THESE CASES}

To investigate whether this interest for gamified cognitive training can be generalized to a larger PD population, a recruitment newsletter was sent out which contained information on various PD research projects. Among others, it presented a brief introduction to a randomized controlled trial on the effects of a gamified cognitive training in PD [8], including 2 clickable buttons directed to the recruitment website. The newsletter was sent on April $3^{\text {rd }} 2017$ at $7 \mathrm{pm}$ to 1103 PD patients in the Netherlands. As early as the next morning, 60 patients requested the patient information brochure via the recruitment website. The email was opened by over 800 patients, and the recruitment website traffic increased by over 7 times within a month. In total, 135 PD patients requested the patient information brochure via this single newsletter and 55 patients applied to the study, underlining that a larger population of PD patients may be interested in using structured and gamified ways to train cognition. The results from this study are now being analyzed and, when published, may add to the current evidence for the effectivity of gamified cognitive training.

\section{DISCUSSION}

\section{PRINCIPAL RESULTS}

The 3 patients presented here are, despite their differences in key characteristics such as age, gender, disease status, and disease duration, also similar in many ways. First, all 3 patients were aware of their decreased cognitive abilities and were proactively looking for ways to potentially counteract their imminent cognitive decline. They challenged themselves with self-invented trainings to improve their own daily life functioning. The first patient used a computer videogame race simulation to train his real-life driving skills. The second patient does not rely on lists to remember meetings or phone numbers, and thereby trains her memory performance for a variety of everyday functions such as shopping. The third patient used an online bridge game to train her concentration and memory performance. Taken together, these 3 stories carefully suggest that at least some PD patients are trying to counteract their 
cognitive deficits with self-invented trainings that address various cognitive skills. Whether such interventions are actually effective requires further formal testing in controlled studies.

Second, all 3 patients incorporated a form of play to address their cognitive deficits. Although the second patient did not resort to a game, the self-invented training approaches of all 3 patients entailed gamified elements (goals, challenges, and reward systems), which likely add motivation to continue the training. The first patient challenges himself to drive faster and faster laps, the second patient is rewarded each time she remembers the grocery list correctly, and the third patient has the goal to win as many tricks as possible. Indeed, in various studies, gamification has been found to increase the motivation and engagement of study subjects [10-12]. Owing to the predictability and repeatability of traditional (nongamified) trainings, eventually patients might get bored, increasing the risk of dropouts $[13,14]$. This could be avoided by challenging patients into performing interventions of varying complexity using attractive, interactive environments. There is debate on the support for gamified training and a large percentage of the general elderly population has never played game interventions [15], but it is unclear whether this is due to a lack of interest or if they are unfamiliar with the concept of gamified training. In exergaming studies, in the field of PD, it has already been established that patients are able to play games, improve their gameplay performance, and, more importantly, enjoy playing exergames $[16,17]$. One specific example was a recent study where gaming elements were used to promote adherence to a home-based exercise intervention; the results showed that PD patients, despite their well-known difficulties to engage in exercise, faithfully adhered to a regime of aerobic exercise at home, precisely as prescribed, namely 3 times a week for $30 \mathrm{~min}$ [18]. Also, various researchers suggested that trainings should be personalized by tailoring the intervention to the individuals' rehabilitation needs and performance levels, thereby improving motivation and adherence $[15,16,19]$. All 3 cases presented above likely showed positive attitudes toward gamification and used gamification strategies, such as goal setting, reinforcement, and the capacity to overcome challenges, that have been scientifically proven to promote health behavior change and thereby influence health outcomes [20]. Additionally, all 3 cases showed aspects of personalization: They chose their own way of training and made personal adjustments in gameplay or goals within their training.

Finally, all patients showed excellent adherence to the training for prolonged periods of time (several years). They were intrinsically motivated to continue, possibly because they felt that the therapy had a positive effect on their functional performance. An extrinsically motivated person requires an external reward to engage in a particular behavior, whereas intrinsic motivation arises from intrinsically rewarding factors. People may follow a training because it makes them feel better (intrinsic motivation) and feeling better may then have external benefits (extrinsic motivation) [21]. Ultimately, motivated people tend to exercise a behavior that is particularly rewarding to them, which may explain why these 3 patients continued to use their self-invented training for many years. However, Case 1 was treated with dopamine agonists, which could have resulted in increased addictive behavior in PD and thus in more 
adherence to the training. Nevertheless, motivation is an important influencer of adherence and it should be an important part of future interventions in this area.

\section{COMPARISON TO PRIOR WORK}

All 3 patients found their own way to train their cognition, but the majority of PD patients are not likely to be able to create such self-invented trainings. However, in some patients, there seems to be a need for a structured way of training cognitive functions. Various traditional cognitive trainings have already been investigated in PD, with small-to-moderate symptomatic effects on cognition, mainly on measures of processing speed, working memory, and executive functions [5,22]. These previous studies had short follow-up periods of maximally 3 months. It would be interesting to see whether these symptomatic effects also persist in the long term and whether the progression of cognitive decline could potentially be delayed (ie, a neuroprotective or disease-modifying effect). However, none of the previous studies investigated the long-term effects on cognition in PD. To date, there is no evidence whether gamified cognitive training can suppress (let alone delay the progression of) cognitive impairment in PD. Many of the investigated cognitive trainings in PD are lacking rewarding gamification stimulants that could stimulate adherence and eventually improve health outcomes even more. Additionally, many cognitive training studies had methodological challenges, such as the lack of solid sample sizes based on reliable power calculations. Importantly, showing that gamified cognitive training has disease-modifying effects that extend beyond mere symptomatic effects is very difficult and calls for specific study designs to separate temporary symptomatic improvement from a more sustained protective effect on actual progression [23].

Some efforts have been made to create gamified cognitive assessments, which may add benefits over traditional assessments in terms of reducing stress related to the formal assessment situation. These gamified assessments are usually relatively simple puzzles with, for example, added sound effects to appear as a game. More importantly, they validate well against traditional cognitive assessments [13]. Gamified cognitive assessments can additionally be used to evaluate the performance and adjust the game's difficulty level accordingly [8]. To our knowledge, no fully gamified cognitive assessments have been investigated in PD to date.

\section{THEORETICAL BASES}

Some theoretical bases have been proposed which promote health behavior when used in gamified treatments [24]. According to the self-determination theory, for example, it is assumed that everyone is driven by autonomy, competence, and relatedness [25]. Within games, autonomy can be implemented via features such as choice and structured reward systems, competence can be implemented via personalized challenges and feedback, and relatedness can be implemented via social elements [24]. For gamified treatments in PD patients, the complexity of the apathy-reward-motivation system must also be recognized [26]. Although the exact relationship is not yet clear, apathy is thought to result from dopaminergic depletion in the ventral striatum, substantia nigra, and ventral tegmental area [26]. Indeed, 
PD patients have a decreased reward sensitivity in an off-dopaminergic medication state [27]. Personalized trainings, with more rewarding elements and interventions that are specifically tailored to their cognitive abilities, will likely improve the self-efficacy of patients. Patients then feel more in control over the events or behaviors with regard to the training, thereby increasing motivation and enhancing resilience to failure [28]. To increase treatment adherence, a potentially ideal cognitive intervention should contain a mix of training elements targeting various cognitive domains but also contain gamified elements. In addition, it is suggested that a personalized challenge level may result in more engagement in the game [29]. Within PD, some computerized cognitive trainings have been investigated, such as RehaCom (computerassisted cognitive rehabilitation) [30], SmartBrain (28 computerized cognitive exercises) [31], NEUROvitalis (computerized exercises training attention, memory, and executive functions) [32], and InSight (5 exercises training information processing speed) [33], but none of these trainings incorporated gamification or personalization. In other populations, positive effects have been found with health games. For example, the NeuroRacer [34], a game-like training that aims to reduce susceptibility to cognitive interference and adapts the difficulty level to the player's performance levels (personalization) showed positive effects on attention, impulsivity, and multitasking in elderly subjects. Recently, positive results were published for the Project: Evo health game that targets cognitive conditions in children with attention deficit hyperactivity disorder (ADHD) [35]. Although Project: Evo is actually a therapy targeting specific neural circuitries involved in attentional control, the intervention feels like a videogame when it is being performed. The researchers found improvements in working memory and attention, but the treatment was also an attractive way to address ADHD, which is promising when it comes to achieving sustained treatment efficacy over time.

\section{RECOMMENDATIONS FOR FUTURE GAMIFIED INTERVENTIONS}

In Table 1, we briefly summarize considerations considering the design and evaluation of future gamified cognitive trainings. This table is based on recommendations from the literature on both game development and evaluation guidelines [36]. 
Table 1. Considerations for developing future gamified cognitive trainings.

\begin{tabular}{|c|c|c|}
\hline Area & Consideration $^{a}$ & Type \\
\hline \multirow[t]{14}{*}{ Gameplay } & Adopt levels of increasing complexity (with achievable goals) & Gameplay \\
\hline & Introduce cognitively demanding aspects slowly & Gameplay \\
\hline & Clear user-interface design (large fonts, bright colors) & User-interface \\
\hline & Include a dynamic difficulty adaptation mechanism (interactive) & Personalization \\
\hline & Personalize training content to individual needs and in real-time & Personalization \\
\hline & Add social elements (eg, play with grandchildren) & Social functions \\
\hline & Add competitive elements (against oneself, computer, or others) & Social functions \\
\hline & Choose actions that are familiar to patients (daily activities) & Gameplay \\
\hline & Think about fun factors (appealing story, graphics, and sounds) & Gameplay \\
\hline & Set long-term goals to help sustain long-term engagement & Engagement \\
\hline & Provide in-game variance (keep game engaging for longer periods) & Engagement \\
\hline & Reinforce positive performance with visual/audio feedback (reward) & Feedback \\
\hline & Avoid negative feedback & Feedback \\
\hline & Be hesitant with negative progress reports (self-monitoring) & Feedback \\
\hline \multirow[t]{5}{*}{ Development } & Integrate validated theories (eg, self-determination, motivation) & Design \\
\hline & Use recent serious game development guidelines [36] & Design \\
\hline & Participate with $\mathrm{PD}^{\mathrm{b}}$ patients and professionals in design/evaluation & Design \\
\hline & Optionally add other neuroplasticity stimulants (eg, exercise) & Design \\
\hline & (Re)evaluate the game with an evaluation protocol [36] & Evaluation \\
\hline \multirow[t]{5}{*}{ Procedural } & Provide crystal-clear and guided instructions & Instructions \\
\hline & Guide the patient through the first level(s) & Instructions \\
\hline & Set clear goals (distinguish game targets vs training targets) & Instructions \\
\hline & Adopt cross-platform availability and plug-and-play technology & Availability \\
\hline & Optionally add group-based, therapist-guided booster sessions & Efficacy \\
\hline \multirow[t]{8}{*}{ Methodological } & Clearly describe the training to aid in replication (publication) & Epidemiology \\
\hline & Compare standardized versus personalized training & Epidemiology \\
\hline & Have a solid sample size & Epidemiology \\
\hline & Report standard measures of disease severity (H\&Yc, UPDRS III $\left.{ }^{\mathrm{d}}\right)$ & Epidemiology \\
\hline & Report standard measures of medication status (LEDD ${ }^{\mathrm{e}}$ ) & Epidemiology \\
\hline & Report standard measures of cognitive status (MoCaf $\mathrm{MMSE}^{\mathrm{g}}$ ) & Epidemiology \\
\hline & Report objective and subjective measures of safety & Epidemiology \\
\hline & Report measures of feasibility and adherence & Epidemiology \\
\hline
\end{tabular}

aNoncomprehensive considerations for gamified cognitive training design (in the field of Parkinson's Disease); not presented in order of priority and obtained from the wider literature [7,13,15,16,19,20,30,36-39]. bPD: Parkinson's Disease.

'H\&Y: Hoehn \& Yahr scale.

'UPDRS III: Unified Parkinson's Disease Rating Scale Part III.

eLEDD: Levodopa equivalent daily dosage.

${ }^{f} \mathrm{MoCa}$ : Montreal Cognitive Assessment.

gMMSE: Mini Mental State Exam. 


\section{CONCLUSIONS}

Taken together, the 3 patients presented here as well as the recruitment results from a gamified and personalized cognitive training trial [8] may justify the development of more structured ways of training cognitive functions in PD, while incorporating elements to increase adherence such as personalization and gamification. Positive results have already been found with gamified trainings in other populations, and the cases described here suggest that PD is also an attractive area to develop and test gamified cognitive trainings. Our 3 patients also demonstrate enormous creativity and laudable resilience despite having PD. However, the majority of PD patients are not likely to be able to create such self-invented trainings. Researchers, health professionals, patients, and the industry should therefore collaborate to develop motivating and targeted cognitive trainings for persons with PD, for which our considerations offered here can be used. The first steps in this direction have already been taken, and several trials are now ongoing $[8,18,40,41]$.

\section{ACKNOWLEDGMENTS}

All authors contributed to the manuscript and approved the final manuscript.

\section{CONFLICTS OF INTEREST}

None declared. 


\section{REFERENCES}

1. Yarnall AJ, Breen DP, Duncan GW, Khoo TK, Coleman SY, Firbank MJ, Nombela C, Winder-Rhodes S, Evans JR, Rowe JB, Mollenhauer B, Kruse N, Hudson G, Chinnery PF, O’Brien JT, Robbins TW, Wesnes K, Brooks DJ, Barker RA, Burn DJ, ICICLE-PD Study Group. Characterizing mild cognitive impairment in incident Parkinson disease The ICICLE-PD Study. Neurology AAN Enterprises; 2014;82(4):308-316. PMID: 24363137

2. Hely MA, Reid WGJ, Adena MA, Halliday GM, Morris JGL. The Sydney multicenter study of Parkinson's disease: The inevitability of dementia at 20 years. Mov. Disord. Wiley-Blackwell; 2008 Feb 28;23(6):837-844. PMID: 18307261

3. Aarsland D, Kurz MW. The epidemiology of dementia associated with Parkinson's disease. Brain Pathol. Wiley/Blackwell (10.1111); 2010 May;20(3):633-639. PMID: 20522088

4. Schmitt FA, Aarsland D, Bronnick KS, Meng X, Tekin S, Olin JT. Evaluating rivastigmine in mild-to-moderate Parkinson's disease dementia using ADAS-cog items. American journal of Alzheimer's disease and other dementias SAGE PublicationsSage CA: Los Angeles, CA; 2010 Aug;25(5):407413. PMID: 20392860

5. Leung IHK, Walton CC, Hallock H, Lewis SJG, Valenzuela M, LampitA. Cognitive training in Parkinson disease: A systematic review and meta-analysis. Neurology 2015 Oct 30;85(21):1843-1851. PMID: 26519540

6. Kalbe E, Folkerts A-K. Cognitive Training in Parkinson's Disease - A New Therapy Option?. Fortschritte der Neurologie-Psychiatrie 2016 Jul;84 Suppl 1(S 01):S24-35. PMID: 27192506

7. Walton CC, Naismith SL, Lampit A, Mowszowski L, Lewis SJG. Cognitive Training in Parkinson's Disease. Neurorehabilitation and neural repair SAGE PublicationsSage CA: Los Angeles, CA; 2017 Mar 1;31(3):207-216. PMID: 27899737

8. Van de Weijer SCF, Duits AA, Bloem BR, Kessels RP, Jansen JFA, Köhler S, Tissingh G, Kuijf ML. The Parkin'Play study: protocol of a phase II randomized controlled trial to assess the effects of a health game on cognition in Parkinson's disease. BMC Neurol BioMed Central; 2016 Nov 3;16(1):209. PMID: 27809791

9. Hoehn MM, Yahr MD. Parkinsonism: onset, progression and mortality. Neurology 1967 May;17(5):427442. PMID: 6067254

10. Prins PJM, Dovis S, Ponsioen A, Brink ten E, van der Oord S. Does computerized working memory training with game elements enhance motivation and training efficacy in children with ADHD? Cyberpsychol Behav Soc; 2011 Mar;14(3):115-122. PMID: 20649448

11. Brown HR, Zeidman P, Smittenaar P, Adams RA, McNab F, Rutledge RB, et al. Crowdsourcing for cognitive science the utility of smartphones. PLoS ONE Public Library of Science; 2014;9(7):e100662. PMID: 25025865

12. Aalbers T, Baars MAE, Olde Rikkert MGM, Kessels RPC. Puzzling with online games (BAM-COG): reliability, validity, and feasibility of an online self-monitor for cognitive performance in aging adults. J. Med. Internet Res.; 2013 Dec 3;15(12):e270. PMID: 24300212 
13. Lumsden J, Edwards EA, Lawrence NS, Coyle D, Munafò MR. Gamification of Cognitive Assessment and Cognitive Training: A Systematic Review of Applications and Efficacy. JMIR Serious Games; 2016 Jul 15;4(2):e11. PMID: 27421244

14. Pachoulakis I, Papadopoulos N, Analyti A. Kinect-Based Exergames Tailored to Parkinson Patients. International Journal of Computer Games Technology Hindawi; 2018 Oct 30;2018(4):1-14.

15. Tobaigy A, Alshehri MA, Timmons S, Helal OF. The feasibility of using exergames as a rehabilitation tool: the attitudes, awareness, opinions and experiences of physiotherapists, and older people towards exergames. J Phys Ther Sci The Society of Physical Therapy Science; 2018 Apr;30(4):555-562. PMID: 29706705

16. Barry G, Galna B, Rochester L. The role of exergaming in Parkinson's disease rehabilitation: a systematic review of the evidence. J Neuroeng Rehabil BioMed Central; 2014 Mar 7;11(1):33. PMID: 24602325

17. Pompeu JE, Mendes FADS, Silva KGD, Lobo AM, Oliveira T de P, Zomignani AP, et al. Effect of Nintendo $\mathrm{Wii}^{\mathrm{TM}}$-based motor and cognitive training on activities of daily living in patients with Parkinson's disease: A randomised clinical trial. Physiotherapy 2012 Sep;98(3):196-204. PMID: 22898575

18. van der Kolk NM, de Vries NM, Penko AL, van der Vlugt M, Mulder AA, Post B, et al. A remotely supervised home-based aerobic exercise programme is feasible for patients with Parkinson's disease: results of a small randomised feasibility trial. Journal of Neurology, Neurosurgery \& Psychiatry; 2017 Jun 12; jnnp-2017-315728. PMID: 28607121

19. Keshner EA. Virtual reality and physical rehabilitation: a new toy or a new research and rehabilitation tool? J Neuroeng Rehabil BioMed Central; 2004 Dec 3;1(1):8. PMID: 15679943

20. Cugelman B. Gamification: what it is and why it matters to digital health behavior change developers. JMIR Serious Games 2013 Dec 12;1(1):e3. PMID: 25658754

21. Medalia A, Saperstein A. The role of motivation for treatment success. Schizophr Bull 2011 Sep;37 Suppl 2(suppl 2):S122-8. PMID: 21860041

22. Glizer D, MacDonald PA. Cognitive Training in Parkinson's Disease: A Review of Studies from 2000 to 2014. Parkinson's Disease Hindawi Publishing Corporation; 2016;2016(4):1-19. PMID: 27688923

23. MCGhee DJM, Ritchie CW, Zajicek JP, Counsell CE. A review of clinical trial designs used to detect a disease-modifying effect of drug therapy in Alzheimer"s disease and Parkinson"s disease. BMC Neurol; 2016 Jun 16;16(1):92. PMID: 27312378

24. Mühlhaus J, Frieg H, Bilda K, Ritterfeld U. Game-Based Speech Rehabilitation for People with Parkinson's Disease. Entertainment Computing; 2017. p. 76-85.

25. Ryan RM, PatrickH, Deci EL, Williams GC. Facilitating health behaviour change and its maintenance: Interventions based on self-determination theory. The European Health Psychologist, 2008 March;10:2-5.

26. Brok den MGHE, van Dalen JW, van Gool WA, Moll van Charante EP, de Bie RMA, Richard E. Apathy in Parkinson's disease: A systematic review and meta-analysis. Mov. Disord. 2015 May 1;30(6):759-769. PMID: 25787145 
27. Muhammed K, Manohar S, Ben Yehuda M, Chong TTJ, Tofaris G, Lennox G, et al. Reward sensitivity deficits modulated by dopamine are associated with apathy in Parkinson's disease. Brain Oxford University Press; 2016 Oct 1;139(10):2706-2721. PMID: 27452600

28. Esposito F, Gendolla GHE, Van der Linden M. Are self-efficacy beliefs and subjective task demand related to apathy in aging? Aging \& Mental Health Routledge; 2014 Mar 12;18(4):521-530. PMID: 24286481

29. Csikszentmihalyi M. Flow. Harper Collins; 2009. ISBN: 9789053525081

30. Cerasa A, Gioia MC, Salsone M, Donzuso G, Chiriaco C, Realmuto S, et al. Neurofunctional correlates of attention rehabilitation in Parkinson's disease: an explorative study. Neurological sciences; 2014 Feb 20;35(8):1173-1180. PMID: 24554416

31. París AP, Saleta HG, la Cruz Crespo Maraver de M, Silvestre E, Freixa MG, Torrellas CP, et al. Blind randomized controlled study of the efficacy of cognitive training in Parkinson's disease. Mov. Disord. Wiley Subscription Services, Inc., A Wiley Company; 2011 Jun;26(7):1251-1258. PMID: 21442659

32. Petrelli A, Kaesberg S, Barbe MT, Timmermann L, Fink GR, Kessler J, et al. Effects of cognitive training in Parkinson's disease: a randomized controlled trial. Parkinsonism and Related Disorders 2014 Nov;20(11):1196-1202. PMID: 25242806

33. Edwards JD, Hauser RA, O’Connor ML, Valdés EG, Zesiewicz TA, Uc EY. Randomized trial of cognitive speed of processing training in Parkinson disease. Neurology Lippincott Williams \& Wilkins; 2013 Oct 8;81(15):1284-1290. PMID: 24014503

34. Anguera JA, Boccanfuso J, Rintoul JL, Al-Hashimi O, Faraji F, Janowich J, et al. Video game training enhances cognitive control in older adults. Nature 2013 Sep 4;501(7465):97-101. PMID: 24005416

35. Davis NO, Bower J, Kollins SH. Proof-of-concept study of an at-home, engaging, digital intervention for pediatric ADHD. Beiki O, editor. PLoS ONE 2018;13(1):e0189749. PMID: 29324745

36. Idriss M, Tannous H, Istrate D, Perrochon A, Salle J-Y, Ho Ba Tho M-C, et al. Rehabilitation-Oriented Serious Game Development and Evaluation Guidelines for Musculoskeletal Disorders. JMIR Serious Games JMIR Publications Inc., Toronto, Canada; 2017 Jul 4;5(3):e14. PMID: 28676468

37. Paraskevopoulos IT, Tsekleves E, Craig C, Whyatt C, Cosmas J. Design guidelines for developing customised serious games for Parkinson's Disease rehabilitation using bespoke game sensors. Entertainment Computing 2014 Dec;5(4):413-424.

38. Dörrenbächer S, Müller PM, Tröger J, Kray J. Dissociable effects of game elements on motivation and cognition in a task-switching training in middle childhood. Front Psychol Frontiers; 2014;5(158):1275. PMID: 25431564

39. Van de Weijer SCF, Hommel ALAJ, Bloem BR, Nonnekes J, De Vries NM. Promising non-pharmacological therapies in PD: Targeting late stage disease and the role of computer based cognitive training. Parkinsonism and Related Disorders 2018 Jan;46 Suppl 1:S42-S46. PMID: 28923292

40. van der Kolk NM, Overeem S, de Vries NM, Kessels RPC, Donders R, Brouwer M, et al. Design of the Park-in-Shape study: a phase II double blind randomized controlled trial evaluating the effects of exercise on motor and non-motor symptoms in Parkinson's disease. BMC Neurol BioMed Central; 2015 Apr 16;15(1):56. PMID: 25880966 
41. Vriend, C; Van Balkom, TD. 2016. Clinical Trial registration for Cognitive Training in Parkinson Study (cogtips). https://clinicaltrials.gov/ct2/show/NCT02920632.

Archived at: http://www.webcitation.org/72EE6EFbZ 


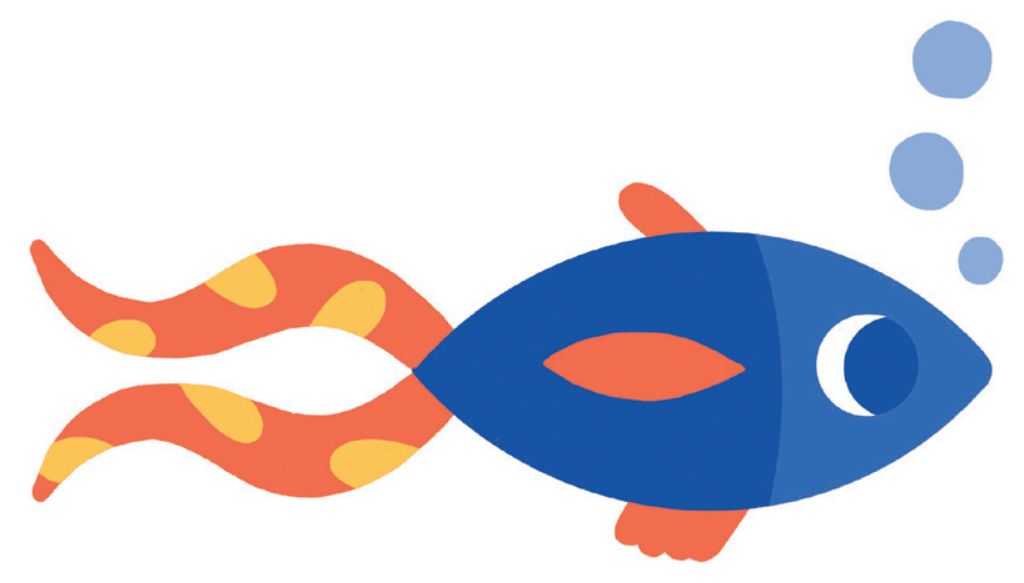




\section{CHAPTER 5}

\section{The validation of a new online cognitive assessment tool: The MyCognition Quotient}




\section{ABSTRACT}

Objectives: Cognitive impairment affects many psychiatric patients, influences daily functioning, and should be an important treatment focus. Assessment of cognitive status is crucial in cognitive remediation studies. However, current test batteries have limitations. A new, online tool, the MyCognition Quotient (MyCQ ${ }^{\mathrm{TM}}$ ), was developed to assess cognition within $30 \mathrm{~min}$. We present the psychometric properties and aim to determine the validity of the MyCQ ${ }^{\mathrm{TM}}$ by comparing it with the Cambridge Neuropsychological Automated Test Battery (CANTAB).

Methods: Eighty-seven patients diagnosed with obsessive compulsive disorder, schizophrenia/ schizoaffective disorder, or major depressive disorder were included. Patients completed both the MyCQ ${ }^{\mathrm{TM}}$ and CANTAB.

Results: Our hypothesized domains of psychomotor speed, attention, episodic memory, working memory, and executive functioning were confirmed by principal component analysis. The MyCQ ${ }^{\text {TM }}$ total score correlated highly with the CANTAB total score. The MyCQ ${ }^{\text {TM }}$ domains of psychomotor speed, attention, and episodic memory showed moderate to high correlations with corresponding CANTAB domains. Working memory and executive functioning had limited divergent validity. Conclusion: The $\mathrm{MyCQ}^{\mathrm{TM}}$ appears to be a promising instrument for assessing cognition online within a mixed psychiatric population. It is cost-efficient, easily administered, and usable in different psychiatric populations, which makes it a good candidate for both clinical and community studies. 


\section{INTRODUCTION}

Cognitive impairment is a common problem in psychiatric patients (Millan et al., 2012), seriously affecting functional outcome and quality of life in patients with a variety of psychiatric disorders (Brissos, Dias, \& Kapczinski, 2008; Green, Kern, \& Heaton, 2004; McCall \& Dunn, 2003; Tolman \& Kurtz, 2012). Treatment of cognitive deficits has received increasing attention, and cognitive remediation programs show promising results for patients with a psychotic disorder (Anaya et al., 2012; Grynszpan et al., 2011; Wykes, Huddy, Cellard, McGurk, \& Czobor, 2011) as well as patients with other psychiatric diagnoses (Motter et al., 2016; Tchanturia, Lounes, \& Holttum, 2014). To further develop and validate these cognitive remediation programs and for successful implementation in the (outpatient) clinic, assessment of the patient's cognitive status is essential. Unfortunately, although many good instruments are available for assessing cognitive functioning, their content, methods, and applications vary widely (Keefe et al., 2004). They require specialist supervision making them timeconsuming and expensive and are usually confined to a clinical setting. A new, web-based tool, the MyCognition Quotient (MyCQ ${ }^{T M}$ ), has been developed to overcome these drawbacks. The aim of this paper is to present the MyCQ ${ }^{\mathrm{TM}}$ and report its psychometric properties.

To measure the full spectrum of cognitive functioning, traditionally, a broad range of separate tests are combined into a neurocognitive test battery. There is an extensive choice of tests available that have proven themselves valid, reliable, and sensitive over the last decades in measuring specific aspects of cognitive functioning. However, the combination of a sufficient number of these traditional tests into a complete test battery can lead to a lengthy assessment, taking up to several hours to complete. This can be burdensome for a patient and affect their motivation and performance, which might even lead to trial dropout. Furthermore, leaving researchers free to create their own test battery can result in a wide variety of test combinations. This negatively influences consistency and generalizability of the results, and thus hinders the standardized evaluation of new interventions aiming to improve cognition. This method also requires test supervisors to be proficient in many different tests. In the last decade, this issue has fortunately received more attention, and a number of guidelines and standard batteries for measuring cognition in psychiatric patients were developed. The Measurement and Treatment Research to Improve Cognition in Schizophrenia (MATRICS) Consensus Cognitive Battery for the assessment of cognitive functioning in schizophrenia (Nuechterlein et al., 2008) could now be considered the gold standard for cognitive assessment in schizophrenia, being recommended by the Food and Drug Administration for clinical trials of cognitive enhancement therapies in schizophrenia. The Brief Assessment of Cognition (BACS; Keefe et al., 2004) is another standardized comprehensive battery for schizophrenia, of which a new version is currently available for affective disorders (BAC-A).

Nowadays, ease of access of test instruments is important for both research as well as clinical practice. Initially, cognitive assessment required the minimum involvement of pen and paper, but often also utilized various props such as colorful blocks (Wechsler, 2008) or 
plastic balls (Culbertson \& Zillmer, 1998). There are currently a number of computerized neurocognitive test batteries available, such as the Cambridge Neuropsychological Test Automated Battery (CANTAB; Cambridge Cognition Ltd., 2008), CogStateBattery (https://www. cogstate.com), Cogtest (https://www.cogtest.com), and CNS vital signs (Gualtieri \& Johnson, 2006). Furthermore, the BACS was recently transformed into a digitalized version in the form of an app that can be used on an iPad (Atkins et al., 2017). These computerized batteries have the advantages of having standardized and consistent administration and automated response recording and scoring, reducing errors in scoring. However, these computerized tests are still dependent on software and hardware that can be relatively expensive, are often tied to a specific location, and still require supervision by a trained expert. Web-based and self-administered assessment would increase accessibility and reduce costs, which could be advantageous for both research as well as clinical practice. In the last years, the mobile, self-administered assessment of cognition has slowly started to make ground, but not many validation studies have yet been done. The THINC-integrated tool (THINC-it) cognitive screener app (McIntyre et al., 2017) has recently been validated for subjects with depression. In the current paper, we study an online and patient-administered instrument for patients with varying psychiatric disorders, the $\mathrm{MyCQ}^{\mathrm{TM}}$.

The MyCQ ${ }^{\mathrm{TM}}$ was developed to assess the broad cognitive status of patients in a relatively quick and easy manner. The instrument was developed with the primary care setting in mind. It is intended as a self- administered tool, to cut down on clinical workload and open up cognitive assessment to larger numbers of patients. Originally, the $\mathrm{MyCQ}^{\mathrm{TM}}$ was intended to be used in unison with an online cognitive training application, where it tracks progress and helps determine which cognitive domains require most training. It furthermore could be used for research purposes as well as in care settings, fulfilling a screening function or to help keep track of cognitive status longitudinally. The $\mathrm{MyCQ}^{\mathrm{TM}}$ aims to assess five cognitive domains through 10 short subtests and can be completed online via PC or iPad. Furthermore, the MyCQ ${ }^{\mathrm{TM}}$ was developed to be used transdiagnostically, without a specific patient population in mind.

In the present study, we assessed the psychometric properties of the $\mathrm{MyCQ}^{\mathrm{TM}}$ in a transdiagnostsic sample. Internal consistency and structure of the instrument were evaluated. Convergent and divergent validity was determined by comparing the MyCQ ${ }^{\mathrm{TM}}$ with the CANTAB. Finally, we examined the ability of the cognitive domains to relate to age and premorbid IQ.

\section{METHODS}

\section{SUBJECTS}

Subjects were recruited between May 2014 and February 2017 at the psychiatry department of the Amsterdam University Medical Centers (location AMC), The Netherlands, as part of a randomized controlled trial investigating the effectiveness of a new web-based cognitive training game. This study was approved by the Medical Ethics Committee of the AMC and 
carried out in accordance with the latest version of the Declaration of Helsinki. Primarily, eligible patients coming for intake or treatment for their psychiatric disorder were invited to participate in the study. Subjects were also recruited by flyers and posters distributed throughout the AMC. To be included in the trial, subjects had to be aged between 16 and 55 and diagnosed with a main Diagnostic and Statistical Manual of Mental Disorders - Fourth Edition (Text Revision) (DSM-IV-TR; American Psychiatric Association, 2000) Axis I disorder of schizophrenia/schizoaffective disorder, obsessive compulsive disorder or major depressive disorder. Exclusion criteria were a high risk of suicide, an unstable comorbid medical disorder, meeting the criteria for a substance use disorder within the last 3 months, a history of a clinically significant abnormality of the neurological system or seizure, and a premorbid IQ below 70. All subjects received a compensation of $€ 40$ and a travel cost reimbursement. Written informed consent was obtained from all subjects and from the legal guardians when the subject was younger than 18 years.

\section{THE MYCQ ${ }^{\mathrm{TM}}$}

The MyCQ ${ }^{\mathrm{TM}}$ was developed to assess cognitive functioning in a fast and efficient way. It is available online and was developed to be self- administered in a variety of settings. The $\mathrm{MyCQ}^{\mathrm{TM}}$ consists of 10 sub-tests that are hypothesized to measure five primary cognitive domains: psychomotor speed, attention, episodic memory, working memory, and executive functioning. Although researchers do not fully agree on how cognitive functioning can be divided into different domains, the MATRICS initiative has identified seven separate cognitive domains (speed of processing, attention/vigilance, working memory, verbal learning and memory, visual learning and memory, reasoning and problem solving, and social cognition) that are often affected in schizophrenia and that are now leading in studies about cognitive function in schizophrenia (Nuechterlein et al., 2004). The MATRICS domains are similar to those of the $\mathrm{MyCQ}^{\mathrm{TM}}$. Although the MyCQ ${ }^{\mathrm{TM}}$ considers episodic memory as one domain, the battery includes a verbal as well as a visual memory task. The $\mathrm{MyCQ}^{\mathrm{TM}}$ does not include a social cognition domain, because it focuses specifically on neurocognitive functioning. Social cognition is recognized as an important construct that is often impaired in schizophrenia (Green \& Leitman, 2008) as well as in mood disorders (Ladegaard, Larsen, Videbech, \& Lysaker, 2014) and is associated with functional impairment (Fett et al., 2011). However, social cognition and nonsocial neurocognitive functioning have been shown to be related but distinct phenomena (Fett et al., 2011; Hasson-Ohayon, Goldzweig, Lavi-Rotenberg, Luther, \& Lysaker, 2018; van Hooren et al., 2008). With the five domains assessed by the $\mathrm{MyCQ}^{\mathrm{T}}$, the primary neurocognitive functions are covered. The $\mathrm{MyCQ}^{\mathrm{TM}}$ uses renowned paradigms that index the key areas of cognitive function. Each subtest is based on a reliable and well validated paper-and-pencil cognitive test. In the selection of the subtests, it was important that subtests were relatively brief and would be easy to use without expert administration. A detailed description of the subtests and their paper-and-pencil equivalents and the corresponding hypothesized domains are presented in Table 1 and Data S1. Written instructions with illustrative pictograms are included in the instrument and are provided prior to each subtest being taken. After the instruction, a short practice session is also provided. Subjects receive feedback on their performance after this 
practice in the form of the number of errors made and reaction speed and then proceed with the actual test. For every subtest, two outcome variables are recorded: mean latency and total number of errors, resulting in a total of 20 outcome variables.

\section{CAMBRIDGE NEUROPSYCHOLOGICAL TEST AUTOMATED BATTERY}

To assess the convergent validity of the MyCQ ${ }^{\text {TM }}$, the CANTAB (Cambridge Cognition Ltd., 2008) was employed as second measurement of cognitive functioning. This computerized test battery has been extensively used in clinical practice as well as in scientific studies in a wide range of disorders and healthy controls. It is mentioned in over 2,000 scientific publications, and its ability to adequately discriminate between healthy adults and various (neuro) psychiatric populations has been confirmed (Egerhazi, Berecz, Bartok, \& Degrell, 2007; Haring, Mottus, Koch, Trei, \& Maron, 2015). It shows moderate correlations with traditional neuropsychological assessments (Smith, Need, Cirulli, Chiba-Falek, \& Attix, 2013). The CANTAB includes a variety of subtests that are delivered on a touchscreen computer and can be combined into different test batteries. We used the five CANTAB sub-tests choice reaction time (CRT), rapid visual information Processing (RVP), verbal recognition memory (VRM), spatial working memory (SWM), and intra-extra dimensional set shift (IED) to measure the five domains of psychomotor speed, attention, episodic memory, working memory, and executive functioning.

Table 1. Individual $M y C Q^{T M}$ tests listed with test equivalents and corresponding cognitive domains

\begin{tabular}{llll}
\multicolumn{1}{l}{ MyCQ } & Proposed domain & Validated test equivalent \\
\hline 1 & Simple reaction time (SRT) & Psychomotor speed/attention & Donders type A \\
\hline 2 & Choice reaction time (CRT) & Psychomotor speed/attention & Donders type B \\
\hline 3 & Go no go reaction time (GNG) & Psychomotor speed/attention & Donders type C \\
\hline 4 & $\begin{array}{l}\text { Verbal memory recognition } \\
\text { (VeMR) }\end{array}$ & Episodic memory & Rey auditory verbal learning test \\
\hline 5 & $\begin{array}{l}\text { Visual memory recognition } \\
\text { (ViMR) }\end{array}$ & Episodic memory & Benton visual retention test \\
\hline 6 & N-back 1 (NB1) & Working memory & One back \\
\hline 7 & N-back2 (NB2) & Working memory & Two back \\
\hline 8 & Coding (COD) & Working memory & Digit symbol substitution test \\
\hline 9 & Trail making test A (TMA) & Executive function & Trail making test part A \\
\hline 10 & Trail making test B (TMB) & Executive function & Trail making test part B
\end{tabular}

Note. MyCQ ${ }^{\mathrm{TM}}$ : MyCognition Quotient.

\section{PREMORBID IQ}

Premorbid intelligence was assessed by using the Dutch version of the National Adult Reading Test (NART; Bright, Jaldow, \& Kopelman, 2002), which is a valid estimate of a person's premorbid level of intellectual ability. The NART is an untimed measure, consisting of 50 words with atypical phonemic pronunciation. Subjects are presented these words on a list and are asked to read each aloud. 


\section{PROCEDURE}

Subjects were assessed as part of a larger randomized controlled trial testing the effectiveness of a new cognitive remediation game, AquaSnap ${ }^{\mathrm{TM}}$. When approached for participation, subjects received written and oral information about the study goals and procedures. After signing informed consent and being negatively screened for the exclusion criteria, subjects were interviewed regarding their clinical, social, and cognitive status. Then, the cognitive assessment began with the NART. Thereafter, the subjects completed the CANTAB sub-tests followed by the MyCQ ${ }^{\mathrm{TM}}$. The MyCQ ${ }^{\mathrm{TM}}$ was completed on a laptop with a mouse. A trained psychology student was present during the full assessment to introduce and supervise tasks and to make sure participants understood the instructions. Total testing time for each subject could take up to $4 \mathrm{hr}$. Because some subjects experienced difficulty sustaining their attention over such a period of time, sometimes two appointments were necessary to complete the measurement. The second appointment was always planned as soon as possible following the first appointment. Subjects could ask for a short break when needed during the testing session.

\section{STATISTICAL ANALYSES}

The underlying structure of the $\mathrm{MyCQ}{ }^{\mathrm{TM}}$ was evaluated by examining intercorrelations and performing a principal component analysis (PCA). Internal consistency for the instrument and its subdomains were measured with Cronbach's coefficient.

Convergent and divergent validity was assessed by calculating Pearson correlations between the five composite domain scores of the $\mathrm{MyCQ}^{\mathrm{TM}}$ and CANTAB. Composite domain scores were computed by averaging the $z$-scores for each domain. Some variables were inversed, so that a higher score meant more impaired cognition. The decision about which outcomes to combine into a composite score was based on factor structure for the MyCQ ${ }^{\mathrm{TM}}$. For the CANTAB, we chose one subtest per cognitive domain and combined the key variables (CRT mean correct latency, RVPA and RVP correct rejections, VRM free recall (short term) and recognition (short and long term), IED total errors, and spatial working memory total errors) into a composite score.

We examined the sensitivity of the $\mathrm{MyCQ}^{\mathrm{TM}}$ to differentiate between in-group differences by examining Pearson correlations between cognitive domain scores and sum scores and NART and age.

All statistical analyses were performed in SPSS (version 22) for Windows, and statistical significance was set at the 0.05 level.

\section{RESULTS}

A total of 87 subjects were included in this study ( 44 male). Age ranged from 16 to 56 years $(M=32.0, S D=10.44)$. The mean total duration of the $\mathrm{MyCQ}^{\mathrm{TM}}$ was $30.8 \mathrm{~min}$ (range $=24.7-64.8$, $S D=6.43 \mathrm{~min}$ ). Table 2 presents the sample's demographic characteristics. Before further analyses, the data were inspected for outliers. Nine MyCQ ${ }^{\mathrm{TM}}$ and five CANTAB data points 
were identified as outliers for falling more than four standard deviations from the mean. These severely deviating data-points most likely occurred due to measurement error (e.g., misplacement of the fingers on the keyboard or starting the subtest before instructions were clear) and were excluded from further analyses. Outliers were found in the $\mathrm{MyCQ}^{\mathrm{TM}}$ variables: simple reaction time total errors, $\mathrm{N}$-back 1 total errors, $\mathrm{N}$-back 2 mean latency, coding mean latency, trail making test A (TMA) mean latency, TMA total errors, and trail making test B (TMB) mean latency and in the CANTAB variables: RVPA, VRM short-term recognition and long-term recognition, IED, and CRT. Almost none of the subjects made errors on the MyCQ ${ }^{\mathrm{TM}} \mathrm{TMA}$ and trail making test B (TMB), so both variables were excluded from further analyses. In Table 2, the means and standard deviations of the $\mathrm{MyCQ}^{\mathrm{TM}}$ variables are presented.

Table 2. Baseline demographic data and $M y C Q^{T M}$ outcomes of the sample, outliers removed

\begin{tabular}{|c|c|c|c|}
\hline Characteristic & & Mean (SD) or $n(\%)$ & Range \\
\hline Age & & $32(10.4)$ & \\
\hline NART & & $99(14.2)$ & \\
\hline Gender & Male & $44(50.6)$ & \\
\hline & Female & $43(49.4)$ & \\
\hline Working? & Yes & $52(59.8)$ & \\
\hline & No & $34(39.1)$ & \\
\hline DSM-IV-TR diagnosis & Psychotic disorder & $36(41.4)$ & \\
\hline & Obsessive-compulsive disorder & $36(41.4)$ & \\
\hline & Depressive disorder & $15(17.2)$ & \\
\hline Level of education completed & Higher tertiary & $27(31.0)$ & \\
\hline & Lower tertiary & $20(23.0)$ & \\
\hline & Secondary & $34(39.1)$ & \\
\hline & Primary & $3(3.4)$ & \\
\hline & None & $2(2.3)$ & \\
\hline & Unknown & $1(1.1)$ & \\
\hline Marital status & Unmarried & $61(70.1)$ & \\
\hline & Married or living together & $21(24.1)$ & \\
\hline & Divorced & $4(4.6)$ & \\
\hline & Unknown & $1(1.1)$ & \\
\hline MyCQ ${ }^{\mathrm{TM}}$ subtest scores & SRT Mean latency & $375.9(61.03)$ & $258-564$ \\
\hline & SRT Total errors & $1.0(1.50)$ & $0-7$ \\
\hline & CRT Mean latency & $466.2(93.79)$ & $313-823$ \\
\hline & CRT Total errors & $1.6(2.85)$ & $0-14$ \\
\hline & GNG Mean latency & $510.4(79.29)$ & $371-793$ \\
\hline & GNG Total errors & $1.4(1.62)$ & $0-6$ \\
\hline & VeMR Mean latency & $931.0(195.79)$ & $582-1462$ \\
\hline
\end{tabular}


Table 2. Continued

Characteristic Mean (SD) or $n(\%) \quad$ Range

\begin{tabular}{lll}
\hline VeMR Total errors & $11.4(7.59)$ & $0-37$ \\
\hline ViMR Mean latency & $848.9(142.69)$ & $544-1202$ \\
\hline ViMR Total errors & $8.8(7.30)$ & $0-33$ \\
\hline NB1 Mean latency & $796.0(220.38)$ & $461-1456$ \\
\hline NB1 Total errors & $2.7(4.12)$ & $0-27$ \\
\hline NB2 Mean latency & $1152.8(338.59)$ & $633-2384$ \\
\hline NB2 Total errors & $8.4(7.15)$ & $0-29$ \\
\hline COD Mean latency & $900.1(217.17)$ & $579-1865$ \\
\hline COD Total errors & $3.1(2.97)$ & $0-13$ \\
\hline TMA Mean latency & $888.4(229.00)$ & $530-1617$ \\
\hline TMA Total errors & $0.2(0.66)$ & $0-3$ \\
\hline TMB Mean latency & $1218.9(399.63)$ & $613-2646$ \\
\hline TMB Total errors & $0.6(1.01)$ & $0-4$
\end{tabular}

Note. Mean latency in milliseconds. SD: standard deviation; NART: national adult reading test; SRT: simple reaction time; CRT: choice reaction time; GNG: go no go reaction time; VeMR: verbal memory recognition; ViMR: visual memory recognition; NB1: N-back 1; NB2: N-back 2; COD: coding; TMA: trail making test A; TMB: trail making test B; MyCQ ${ }^{\mathrm{TM}}$ : MyCognition Quotient.

\section{STRUCTURE OF THE SCALE AND INTERNAL CONSISTENCY}

To explore the underlying structure of the $\mathrm{MyCQ}^{\mathrm{TM}}$, a PCA with oblique rotation was used on the 18 outcome measures. First, the correlation matrix was inspected to check appropriateness of the data. There were no variables that did not correlate with any other variable or that showed very high correlations with other variables. The determinant of the matrix was 6.972E5 , meaning that there are no indications for multicollinearity in the data. Five components with eigenvalues greater than one were revealed. This model explained $68.0 \%$ of the total variance and largely confirmed our hypothesized domains of psychomotor speed, attention, episodic memory, working memory, and executive functioning. Four variables did not seem to be a perfect fit in the model. GNG total errors, N-back 1 mean latency, and coding total errors did not show a high loading of $>0.45$ on any of the components. Furthermore, $\mathrm{N}$-back 2 Total errors showed similar loadings on two components (episodic memory and executive functioning). Repeating the PCA without these four variables resulted in the final five-component model that had a good fit, including 14 variables. All included components had an eigenvalue above one; every variable presented a high loading on one component only; and the model explained $75.2 \%$ of the total variance and confirmed our hypothesized cognitive domains. Factor loadings and intercorrelations of the components of the final PCA are presented in Table 3.

Internal consistency of the total $\mathrm{MyCQ}^{\mathrm{TM}}$, including all $18 \mathrm{MyCQ}^{\mathrm{TM}}$ variables, was good, with a Cronbach's $a$ of 0.79 . Internal consistency without the four items that were removed due to 
their low factor loadings was slightly smaller, with a Cronbach's a of 0.76. Cronbach's a was also computed for the specific cognitive domains as resulting from the PCA. These statistics were as follows: psychomotor speed, 0.86 ; attention, 0.37 ; episodic memory, 0.78 ; working memory, 0.73; and executive functioning, 0.69 .

\section{CONVERGENT AND DIVERGENT VALIDITY}

One subject was missing CANTAB data, this subject was excluded from further analyses. The MyCQ ${ }^{\text {TM }}$ composites based on the PCA were compared with CANTAB composites using Pearson correlations. The results are presented in Table 4. Significant correlations were found between every matched domain, with the highest correlations between psychomotor speed $(p<0.001)$ and episodic memory $(p<0.001)$. All $z$-scores were also summed to form one MyCQ ${ }^{\text {TM }}$ or CANTAB sum score and were highly correlated $(\mathrm{N}=77, \mathrm{r}=0.650, \mathrm{p}<0.001)$ as can be seen in Figure 1 . For psychomotor speed and attention, divergent validity appeared to be reasonable, with lower correlations between nonmatching domains compared with the matching domains of CANTAB and MyCQ ${ }^{\text {TM }}$. Although the MyCQ ${ }^{\text {TM }}$ domains of episodic memory, working memory, and executive functioning showed significant correlations with their corresponding CANTAB domains, these components showed comparable or stronger relationships with nonmatching CANTAB domains. 
Table 3. Factor structure of the $\mathrm{MyCQ}^{\mathrm{TM}}$ outcome measures

Final five-component model

\begin{tabular}{|c|c|c|c|c|c|}
\hline & $\begin{array}{l}\text { Psychomotor } \\
\text { speed }\end{array}$ & Attention & $\begin{array}{l}\text { Episodic } \\
\text { memory }\end{array}$ & $\begin{array}{l}\text { Working } \\
\text { memory }\end{array}$ & $\begin{array}{l}\text { Executive } \\
\text { functioning }\end{array}$ \\
\hline SRT mean latency & -0.917 & & & & \\
\hline CRT mean latency & -0.664 & & & & \\
\hline GNG mean latency & -0.899 & & & & \\
\hline SRT total errors & & 0.840 & & & \\
\hline CRT total errors & & 0.721 & & & \\
\hline VeMR total errors & & & 0.875 & & \\
\hline ViMR total errors & & & 0.904 & & \\
\hline VeMR mean latency & & & & -0.939 & \\
\hline ViMR mean latency & & & & -0.883 & \\
\hline NB1 total errors & & & & & 0.648 \\
\hline NB2 mean latency & & & & -0.689 & \\
\hline COD mean latency & & & & & 0.680 \\
\hline TMA mean latency & & & & & 0.676 \\
\hline TMB mean latency & & & & & 0.839 \\
\hline Variance explained & 7.9 & 9.1 & 11.8 & 33.6 & 12.9 \\
\hline
\end{tabular}

Correlations among components

Psychomotor speed

\begin{tabular}{llllll}
\hline Attention & 0.198 & - & & & \\
\hline Episodic memory & $0.314^{\mathrm{a}}$ & 0.191 & - & & \\
\hline Working memory & $0.480^{\mathrm{a}}$ & 0.091 & 0.123 & - & \\
\hline Executive functioning & $0.437^{\mathrm{a}}$ & $0.220^{\mathrm{b}}$ & 0.103 & $0.312^{\mathrm{a}}$ & - \\
\hline MyCQ $^{\text {TM }}$ sum score & $0.774^{\mathrm{a}}$ & $0.446^{\mathrm{a}}$ & $0.538^{\mathrm{a}}$ & $0.742^{\mathrm{a}}$ & $0.619^{\mathrm{a}}$
\end{tabular}

Note. SRT: simple reaction time; CRT: choice reaction time; GNG: go no go reaction time; VeMR: verbal memory recognition; ViMR: visual memory recognition; NB1: N-back 1; NB2: N-back 2; COD: coding; TMA: trail making test A; TMB: trail making test B; MyCQ ${ }^{\text {TM}: ~ M y C o g n i t i o n ~ Q u o t i e n t . ~ P a t t e r n ~ m a t r i x ~ a n d ~}$ correlations between $M y C C^{\mathrm{TM}}$ domains. Principal Component Analyses with Oblimin rotation and Kaiser Normalization. Factor loadings $<0.4$ are hidden. Rotation converged in 12 iterations.

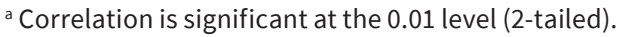

${ }^{\mathrm{b}}$ Correlation is significant at the 0.05 level (2-tailed).

\section{ASSOCIATIONS BETWEEN MYCQ ${ }^{\text {TM }}$ SCORES AND SUBJECT CHARACTERISTICS}

Pearson correlations were calculated between MyCQ ${ }^{\mathrm{TM}}$ scores and age and NART with a significance level of 0.05 . Higher age related to slower psychomotor speed $(r=0.224, p=0.037)$ and worse performance on the working memory $(r=0.232, p=0.033)$ and executive functioning $(r=0.393, p<0.001)$. A higher NART score was associated with better MyCQ ${ }^{\text {TM }}$ performance in psychomotor speed $(r=-0.217, p=0.044)$ and executive functioning $(r=-0.254, p=0.019)$. 
In contrast, when comparing the CANTAB domain scores with these subject characteristics, only two significant correlations were found. The CANTAB sum score $(r=0.229, p=0.038)$ and psycho-motor speed domain ( $r=0.223, p=0.040$ ) were significantly related to age, with higher age relating to worse performance. There were no associations between NART score and CANTAB domain or sum score.

Table 4. Convergent and divergent validity: Pearson correlations between $\mathrm{MyCQ}^{\mathrm{TM}}$ and CANTAB domains

\section{CANTAB Domains}

\begin{tabular}{|c|c|c|c|c|c|c|}
\hline & & \\
\hline & & $\begin{array}{l}\text { Psychomotor } \\
\text { speed }\end{array}$ & Attention & $\begin{array}{l}\text { Episodic } \\
\text { memory }\end{array}$ & $\begin{array}{l}\text { Working } \\
\text { memory }\end{array}$ & $\begin{array}{l}\text { Executive } \\
\text { functioning }\end{array}$ \\
\hline \multirow[t]{5}{*}{$\begin{array}{l}\text { MyCQ }^{\mathrm{TM}} \\
\text { Domains }\end{array}$} & $\begin{array}{l}\text { Psychomotor } \\
\text { speed }\end{array}$ & $0.604^{\mathrm{a}}$ & $0.311^{\mathrm{a}}$ & 0.192 & $0.328^{\mathrm{a}}$ & $0.243^{b}$ \\
\hline & Attention & -0.061 & $0.224^{b}$ & 0.065 & 0.182 & 0.172 \\
\hline & Episodic memory & 0.194 & $0.374^{\mathrm{a}}$ & $0.374^{\mathrm{a}}$ & $0.353^{a}$ & $0.319^{a}$ \\
\hline & Working memory & $0.371^{\mathrm{a}}$ & $0.245^{\mathrm{b}}$ & 0.123 & $0.229^{b}$ & 0.089 \\
\hline & $\begin{array}{l}\text { Executive } \\
\text { functioning }\end{array}$ & $0.360^{a}$ & $0.432^{a}$ & -0.066 & $0.442^{a}$ & $0.278^{b}$ \\
\hline
\end{tabular}

Note. CANTAB: Cambridge Neuropsychological Automated Test Battery; MyCQ ${ }^{T M}$ : MyCognition Quotient. Values printed in bold style correspond to convergent validity, values printed in regular style correspond to divergent validity.

${ }^{\text {a }}$ Correlation is significant at the 0.01 level (2-tailed).

${ }^{\mathrm{b}}$ Correlation is significant at the 0.05 level (2-tailed).

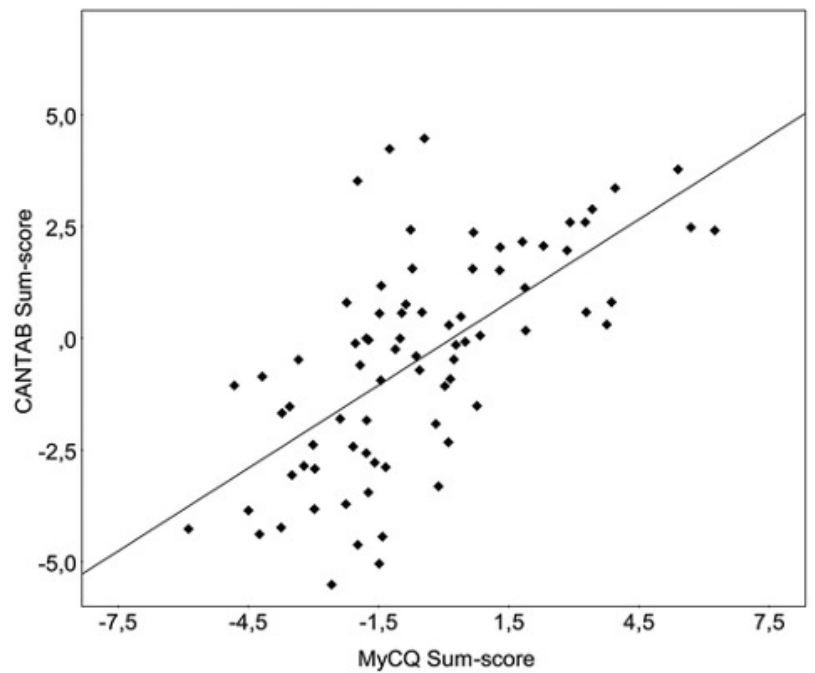

Figure 1. Scatterplot of the relation between MyCognition Quotient (MyCQ ${ }^{\mathrm{TM}}$ ) and Cambridge Neuropsychological Automated Test Battery (CANTAB) sum scores $(r=0.664)$ 


\section{DISCUSSION}

The results of the current study provide initial evidence that the $M y C \mathrm{CQ}^{\mathrm{TM}}$ is a valid and consistent instrument for a relatively quick assessment of cognitive functioning within a transdiagnostic psychiatric population. The instrument measures overall cognition within a relatively short time compared with more traditional instruments and can be self-administered and accessed online, creating possibilities for increased accessibility and reduced costs.

The underlying structure of the $\mathrm{MyCQ}^{\mathrm{TM}}$ confirmed the hypothesized key cognitive domains reasonably well. The $\mathrm{MyCQ}^{\mathrm{TM}}$ subtests load into five separate components, which can be interpreted as the domains of psychomotor speed, attention, episodic memory, working memory, and executive functioning. Although no true consensus exists about which domains of cognition should be considered key domains, a few initiatives have attempted to bring more clarity. The DSM-5 defines six key domains of cognitive functioning (perceptual-motor function, language, learning and memory, social cognition, complex attention, and executive functioning; Sachdev et al., 2014), while the MATRICS initiative identified seven domains on which schizophrenia patients are impaired, including speed of processing, attention, working memory, verbal memory and visual memory, reasoning and problem solving, and social cognition (Nuechterlein et al., 2004). Although these domains do not fully conform to the domains of the $\mathrm{MyCQ}^{\mathrm{TM}}$, there is much overlap, and the $\mathrm{MyCQ}^{\mathrm{TM}}$ includes tests that measure almost all of these domains. The five domains covered by the $\mathrm{MyCQ}^{\mathrm{TM}}$ should provide an adequate quick overall screening for neurocognitive functioning.

During the PCA, we dropped a few items that did not fit in well in the model. Fourteen of the $20 \mathrm{MyCQ}^{\mathrm{TM}}$ outcome measures were eventually included in the final analyses. Most of these measures provide useful information, but the total number of errors of the TMT A and TMT B are an exception, for almost no errors were made on these subtests. In addition, errors on either of these subtests would probably result in a longer mean latency, indicating that this variable can safely be dropped from further analyses. Four more outcome measures were excluded due to either not loading highly on any of the retained factors or loading similarly high on more than one factor. Removing outcome measures that complicate the factor structure of an instrument is common practice. Simple structure, in which each item loads relatively strong on one factor and small on others, helps to achieve easy and meaningful interpretation (Thurstone, 1947). All subtests are still incorporated in the final $\mathrm{MyCQ}^{\mathrm{TM}}$ total score and subdomains, providing evidence that the five cognitive domains are covered by the 10 subtests of the MyCQ ${ }^{\mathrm{TM}}$. More than $75 \%$ of the total variance observed was explained, a good result compared with other studies (Henson \& Roberts, 2006). Nevertheless, our sample was quite small for this type of analysis, which could be of influence on the accuracy and generalizability of the model. Although opinions on minimum sample sizes for PCA differ, most rules of thumb ask for at least 10:1 ratio of $\mathrm{N}$ to variables (Osborne \& Costello, 2004). Until the current results are replicated in a larger study population, caution in the interpretation of these domains is warranted. 
Most MyCQ ${ }^{\mathrm{TM}}$ domains are moderately associated with each other. This finding is not surprising and has been noted by scientists since the beginning of the 20th century when Charles Spearmen first proposed the existence of a general intelligence factor, or $\mathrm{g}$ factor (Spearman, 1904). Furthermore, some of the subtests also load on different domains. For example, different outcome measures of the verbal and visual memory subtests are used for both the working memory and the episodic memory domain. The high association between working memory and psychomotor speed can be explained by both domains relying heavily on the mean latency of subtests.

Comparing the $\mathrm{MyCQ}^{\mathrm{TM}}$ with the CANTAB shows reasonable validity of the instrument. Sum scores of the MyCQ ${ }^{\mathrm{TM}}$ and CANTAB strongly relate to each other. All MyCQ ${ }^{\mathrm{TM}}$ domains show significant associations with corresponding CANTAB domains, with the strongest association between the psychomotor speed domains. This domain consists of the mean latency variables of the reaction time tasks (simple, choice, and go-no-go). MyCQ ${ }^{\mathrm{TM}}$ psychomotor speed relates to the CANTAB domains of attention, working memory, and executive functioning, but not as well as it does to the CANTAB psychomotor speed domain. Attention is covered by both the errors on the simple and choice reaction time task. Within the MyCQ ${ }^{\mathrm{TM}}$, it appears to be especially specific, showing only a significant association with CANTAB attention, while the associations with any of the other CANTAB domains were very small. The total numbers of errors on the verbal and visual memory tests form the episodic memory domain. MyCQ ${ }^{\mathrm{TM}}$ episodic memory shows comparable associations of moderate strength with CANTAB episodic memory and attention. CANTAB working memory and executive functioning show somewhat smaller associations with $\mathrm{MyCQ}^{\mathrm{TM}}$ episodic memory. CANTAB psychomotor speed is not significantly related to $\mathrm{MyCQ}^{\mathrm{TM}}$ episodic memory. The working memory component includes four variables: the mean latency of the two memory tasks, the mean latency of the 2-back task, and the errors on the coding task. This domain relates to the corresponding CANTAB domain, although this relationship is quite weak. Somewhat stronger associations exist with the domains of attention and psychomotor speed. The moderate correlation between working memory and CANTAB psychomotor speed might be explained by the fact that $\mathrm{MyCQ}^{\mathrm{TM}}$ working memory is largely based on mean latency outcome measures. Curiously, $M_{Y C Q}{ }^{\mathrm{TM}}$ working memory and CANTAB executive functioning are not related, although previous studies suggest that these are overlapping concepts (Chan, Shum, Toulopoulou, \& Chen, 2008; McCabe, Roediger, McDaniel, Balota, \& Hambrick, 2010), and the MyCQ ${ }^{\mathrm{TM}}$ also shows a moderate association between the two. Finally, the errors on the 1-back task, the mean latency of the coding task, and both trail making tests' mean latencies are included to form the executive functioning domain. $\mathrm{MyCQ}^{\mathrm{TM}}$ executive functioning has a significant but small correlation with the corresponding CANTAB domain. Most other CANTAB domains are moderately associated with MyCQ ${ }^{\mathrm{TM}}$ executive functioning.

Although the $\mathrm{MyCQ}^{\mathrm{TM}}$ domains of psychomotor speed, attention, and episodic memory show decent divergent validity, this appears less the case for the domains of working memory and executive functioning. The concepts of executive functioning (and to a lesser extent 
working memory) are more complicated, broad and open to conjecture, than the concepts of attention, motor speed or episodic memory. As higher order domains, the structure of executive functioning and working memory are more difficult to capture, because they encompass a range of different components that can even be quite fractioned (Chan et al., 2008). In the current study, only one CANTAB subtest was used per cognitive domain. Although the CANTAB executive functioning domain in this study captured set-shifting, the MyCQ ${ }^{\text {TM }}$ domain consisted of four subtests, measuring different aspects of executive functioning. In a previous study, executive functions appeared to exist out of three latent structures, including one that was considered to be working memory (Lehto, Juujarvi, Kooistra, \& Pulkkinen, 2003). Furthermore, a recent study showed that the CANTAB subtests themselves do not distinguish well between four cognitive domains found in traditional neurocognitive tests (Lenehan, Summers, Saunders, Summers, \& Vickers, 2016). This highlights the overall difficulty of measuring human cognition. The poor divergent validity of the domains of working memory and executive functioning warrants some caution when interpreting the results of the $\mathrm{MyCQ}^{\mathrm{TM}}$. Although using the $\mathrm{MyCQ}^{\text {TM }}$ for assessing global cognitive functioning by using the overall score seems valid, for the confirmation of impairments in the separate domains-especially those of working memory and executive function-additional tests might be considered.

The MyCQ ${ }^{T M}$ is somewhat sensitive to differences in age and premorbid IQ. Better MyCQ ${ }^{\mathrm{TM}}$ psychomotor speed and executive functioning scores are associated with a lower age and a higher premorbid IQ. Working memory also declines with age. In contrast, the CANTAB domains are not associated with premorbid IQ and only the sum score and psychomotor speed show age-related decline. When using the Bonferroni correction for multiple comparisons, only the association between age and executive functioning retains its significance. However, the necessity to Bonferroni correct has been disputed. Especially with a relatively small sample size such as in our study, the probability of Type II errors greatly increases with this method, while the power to find small effects is reduced (Nakagawa, 2004). The effect-size remains the same, however.

This study has a number of additional limitations. To further validate the $\mathrm{MyCQ}^{\mathrm{TM}}$, it should be compared with a wider array of different (both computerized and paper-and-pencil) neurocognitive tests. Specifically, for further establishing concurrent validity, the MyCQ ${ }^{\mathrm{TM}}$ subtests should be compared with their paper-and-pencil equivalents. Second, no group of healthy controls and only patients with three different psychiatric disorders were included in this study. For an instrument that aims to be universal and used across a broad population, more subpopulations should be studied. However, the results do show that the MyCQ ${ }^{\mathrm{TM}}$ can be used to assess the five cognitive domains in the psychiatric conditions we studied. Because we did not include any healthy controls, we are unable to determine if patients are impaired. Administration did not involve counter- balancing. Therefore, order effects were not controlled for and might have influenced the scores on the $\mathrm{MyCQ}^{\text {TM }}$. However, because the scores were primarily used for correlation analyses without evaluating the raw scores, this should not have much effect on the presented findings. Finally, this study only determined a proportion of the 
psychometric properties of the MyCQ ${ }^{\mathrm{TM}}$. Studies aiming to assess the sensitivity to change or test-retest reliability are in progress. Further studies should also investigate if the $\mathrm{MyCQ}^{\mathrm{TM}}$ can predict real-life cognitive functioning, clinical symptoms, or psychosocial functioning.

Despite these limitations, the advantages of assessing cognition with an online assessment tool such as the $\mathrm{MyCQ}^{\mathrm{TM}}$ are numerous. Computerized tests make consistent and precise administration and scoring possible, reducing measurement error and examiner bias. Computerized testing has been used before, but offering it through an online portal is a new development and could be the next step in neurocognitive assessment. One of the important advantages of self-administered and online assessment, over and above the cost- efficiency, is that it enables large scale assessment in a range of settings, both in the clinic and the community. This could be especially important for prevention studies and interventions. There is evidence that cognitive impairment can precede other clinical symptoms. This is especially true for psychotic disorders (Fuller et al., 2002), but might also be the case for mood disorders although results are still inconclusive (Allott, Fisher, Amminger, Goodall, \& Hetrick, 2016). Neurocognitive functioning might well be a shared risk factor for overall mental health and global functioning. Although more studies are necessary to enlighten how cognition influences later functioning, early detection of impairment in neurocognitive functioning might be important and could be widely implemented with an online instrument. However, the usability of the MyCQTM for psychiatric patients in settings outside the clinic should be further established. The $\mathrm{MyCQ}^{\mathrm{TM}}$ already has been used to assess large school populations of 600- 800 students, confirming its usability in community studies for healthy populations (Ratto, Cliveden, \& Sparrowhawk, 2017).

The MyCQ ${ }^{\text {TM }}$ opens up the possibility of home assessment, which might especially be cost and time efficient and less burdensome for patients. The current paper did not investigate assessment at home, and it is still unclear how home assessment might affect the validity and reliability of the results. Uncontrolled confounders, such as distractions, assistance, or substance use, might interfere with reliable assessment. However, in some situations, home assessment might even provide an ecologically more valid estimation of a patient's cognitive functioning in their daily life. The effects on the reliability and validity of the MyCQ ${ }^{\mathrm{TM}}$ when assessing cognition at home should be further investigated. A number of subjects in this study also completed a follow-up measurement of the $\mathrm{MyCQ}^{\mathrm{TM}}$ at home without problems, providing first support for the usability of the instrument for home assessment.

In summary, the $\mathrm{MyCQ}^{\mathrm{TM}}$ appears to be a promising instrument for assessing cognitive functioning online within a mixed psychiatric population. It compares reasonably well with the CANTAB, and its short duration and self-administration capability potentially reduces health care costs. More studies with different populations, for example, Parkinson's disease (van de Weijer et al., 2016) and breast cancer patients with depression, and different neuropsychological assessment batteries are in progress to further validate the $\mathrm{MyCQ}^{\mathrm{TM}}$ and investigate its usability within other populations. 


\section{ACKNOWLEDGEMENTS}

This study was partly funded by MyCognition, London, UK. The sponsor had no role in the data collection, analysis, or interpretation, and in writing the manuscript. This study was also supported by a Top Sector Life Sciences \& Health TKI allowance (LSHM13007). 


\section{REFERENCES}

1. Allott, K., Fisher, C. A., Amminger, G. P., Goodall, J., \& Hetrick, S. (2016). Characterizing neurocognitive impairment in young people with major depression: State, trait, or scar? Brain and Behavior: A Cognitive Neuroscience Perspective, 6(10), e00527. https://doi.org/10.1002/brb3.527

2. American Psychiatric Association (2000). Diagnostic and statistical manual of mental disorders (4th ed.). Washington, DC: American Psychiatric Association.

3. Anaya, C., Martinez Aran, A., Ayuso-Mateos, J. L., Wykes, T., Vieta, E., \& Scott, J. (2012). A systematic review of cognitive remediation for schizo-affective and affective disorders. Journal of Affective Disorders, 142(1-3), 13-21. https://doi.org/10.1016/j.jad.2012. 04.020

4. Atkins, A. S., Tseng, T., Vaughan, A., Twamley, E. W., Harvey, P., Patterson, T., ... Keefe, R. S. (2017). Validation of the tablet-administered brief assessment of cognition (BAC App). Schizophrenia Research, 181, 100-106. https://doi.org/10.1016/j.schres.2016.10.010

5. Bright, P., Jaldow, E., \& Kopelman, M. D. (2002). The national adult reading test as a measure of premorbid intelligence: A comparison with estimates derived from demographic variables. Journal of the International Neuropsychological Society, 8(6), 847-854. https://doi.org/10.1017/ S1355617702860131

6. Brissos, S., Dias, V. V., \& Kapczinski, F. (2008). Cognitive performance and quality of life in bipolar disorder. The Canadian Journal of Psychiatry, 53(8), 517-524. https://doi.org/10.1177/070674370805300806

7. Cambridge Cognition Ltd. (2008). CANTAB Topic: Test-retest reliabilities and detecting reliable change.: CANTAB Resources.

8. Chan, R. C., Shum, D., Toulopoulou, T., \& Chen, E. Y. (2008). Assessment of executive functions: Review of instruments and identification of critical issues. Archives of Clinical Neuropsychology, 23(2), 201-216. https:// doi.org/10.1016/j.acn.2007.08.010

9. Culbertson, W. C., \& Zillmer, E. A. (1998). The Tower of London (DX): A standardized approach to assessing executive functioning in children. Archives of Clinical Neuropsychology, 13(3), 285-301. https://doi.org/ 10.1093/arclin/13.3.285

10. Egerhazi, A., Berecz, R., Bartok, E., \& Degrell, I. (2007). Automated Neuropsychological Test Battery (CANTAB) in mild cognitive impairment and in Alzheimer's disease. Progress in Neuro-Psychopharmacology and Biological Psychiatry, 31(3), 746-751. https://doi.org/10.1016/j. pnpbp.2007.01.011

11. Fett, A. K., Viechtbauer, W., Dominguez, M. D., Penn, D. L., van Os, J., \& Krabbendam, L. (2011). The relationship between neurocognition and social cognition with functional outcomes in schizophrenia: A meta-analysis. Neuroscience and Biobehavioral Reviews, 35(3), 573-588. https://doi.org/10.1016/j. neubiorev.2010.07.001

12. Fuller, R., Nopoulos, P., Arndt, S., O’Leary, D., Ho, B. C., \& Andreasen, N. C. (2002). Longitudinal assessment of premorbid cognitive functioning in patients with schizophrenia through examination of standardized scholastic test performance. American Journal of Psychiatry, 159(7), 1183-1189. https://doi.org/10.1176/appi.ajp.159.7.1183 
13. Green, M. F., Kern, R. S., \& Heaton, R. K. (2004). Longitudinal studies of cognition and functional outcome in schizophrenia: Implications for MATRICS. Schizophrenia Research, 72(1), 41-51. https:// doi.org/ 10.1016/j.schres.2004.09.009

14. Green, M. F., \& Leitman, D. I. (2008). Social cognition in schizophrenia. Schizophrenia Bulletin, 34(4), 670-672. https://doi.org/10.1093/ schbul/sbn045

15. Grynszpan, O., Perbal, S., Pelissolo, A., Fossati, P., Jouvent, R., Dubal, S., \& Perez-Diaz, F. (2011). Efficacy and specificity of computer-assisted cognitive remediation in schizophrenia: A meta-analytical study. Psychological Medicine, 41(1), 163-173. https://doi.org/10.1017/ S0033291710000607

16. Gualtieri, C. T., \& Johnson, L. G. (2006). Reliability and validity of a computerized neurocognitive test battery, CNS vital signs. Archives of Clinical Neuropsychology, 21(7), 623-643. https://doi.org/10.1016/j. acn.2006. 05.007

17. Haring, L., Mottus, R., Koch, K., Trei, M., \& Maron, E. (2015). Factorial validity, measurement equivalence and cognitive performance of the Cambridge Neuropsychological Test Automated Battery (CANTAB) between patients with first-episode psychosis and healthy volunteers. Psychological Medicine, 45(9), 1919-1929. https://doi.org/10.1017/ S0033291714003018

18. Hasson-Ohayon, I., Goldzweig, G., Lavi-Rotenberg, A., Luther, L., \& Lysaker, P. H. (2018). The centrality of cognitive symptoms and metacognition within the interacting network of symptoms, neurocognition, social cognition and metacognition in schizophrenia. Schizophrenia Research https:// doi.org/10.1016/j.schres.2018.07.007, 202, 260-266.

19. Henson, R. K., \& Roberts, J. K. (2006). Use of exploratory factor analysis in published researchCommon errors and some comment on improved practice. Educational and Psychological Measurement, 66(3), 393-416. https://doi.org/10.1177/0013164405282485

20. Keefe, R. S. E., Goldberg, T. E., Harvey, P. D., Gold, J. M., Poe, M., \& Coughenour, L. (2004). The brief assessment of cognition in schizophrenia: Reliability, sensitivity, and comparison with a standard neurocognitive battery. Schizophrenia Research, 67(1), 263-263.

21. Ladegaard, N., Larsen, E. R., Videbech, P., \& Lysaker, P. H. (2014). Higher-order social cognition in first-episode major depression. Psychiatry Research, 216(1), 37-43. https://doi.org/10.1016/j. psychres.2013. 12.010

22. Lehto, J. E., Juujarvi, P., Kooistra, L., \& Pulkkinen, L. (2003). Dimensions of executive functioning: Evidence from children. British Journal of Developmental Psychology, 21, 59-80. https://doi. org/10.1348/026151003321164627

23. Lenehan, M. E., Summers, M. J., Saunders, N. L., Summers, J. J., \& Vickers, J. C. (2016). Does the Cambridge Automated Neuropsychological Test Battery (CANTAB) distinguish between cognitive domains in healthy older adults? Assessment, 23(2), 163-172. https://doi.org/10.1177/ 1073191115581474

24. McCabe, D. P., Roediger, H. L., McDaniel, M. A., Balota, D. A., \& Hambrick, D. Z. (2010). The relationship between working memory capacity and executive functioning: Evidence for a common executive attention construct. Neuropsychology, 24(2), 222-243. https://doi.org/10.1037/a0017619 
25. McCall, W. V., \& Dunn, A. G. (2003). Cognitive deficits are associated with functional impairment in severely depressed patients. Psychiatry Research, 121(2), 179-184. https://doi.org/10.1016/j.psychres. 2003.09 .003

26. McIntyre, R. S., Best, M. W., Bowie, C. R., Carmona, N. E., Cha, D. S., Lee, Y., ... Harrison, J. (2017). The THINC-Integrated tool (THINC-it) screening assessment for cognitive dysfunction: Validation in patients with major depressive disorder. Journal of Clinical Psychiatry, 78(7), 873-881. https:// doi.org/10.4088/JCP.16m11329

27. Millan, M. J., Agid, Y., Brune, M., Bullmore, E. T., Carter, C. S., Clayton, N. S., ... Young, L. J. (2012). Cognitive dysfunction in psychiatric disorders: Characteristics, causes and the quest for improved therapy. Nature Reviews Drug Discovery, 11(2), 141-168. https://doi.org/10.1038/ nrd3628

28. Motter, J. N., Pimontel, M. A., Rindskopf, D., Devanand, D. P., Doraiswamy, P. M., \& Sneed, J. R. (2016). Computerized cognitive training and functional recovery in major depressive disorder: A meta-analysis. Journal of Affective Disorders, 189, 184-191. https://doi.org/10.1016/j.jad.2015.09.022

29. Nakagawa, S. (2004). A farewell to Bonferroni: The problems of low statistical power and publication bias. Behavioral Ecology, 15(6), 1044-1045. https://doi.org/10.1093/beheco/arh107

30. Nuechterlein, K. H., Barch, D. M., Gold, J. M., Goldberg, T. E., Green, M. F., \& Heaton, R. K. (2004). Identification of separable cognitive factors in schizophrenia. Schizophrenia Research, 72(1), 29-39. https://doi.org/ 10.1016/j.schres.2004.09.007

31. Nuechterlein, K. H., Green, M. F., Kern, R. S., Baade, L. E., Barch, D. M., Cohen, J. D., ... Marder, S. R. (2008). The MATRICS consensus cognitive battery, part 1: Test selection, reliability, and validity. American Journal of Psychiatry, 165(2), 203-213. https://doi.org/10.1176/appi. ajp.2007.07010042

32. Osborne, J. W., \& Costello, A. B. (2004). Sample size and subject to item ratio in principal components analysis. Practical Assessment, Research \& Evaluation, 9(11). Available online: http://PAREonline. net/getvn. asp? $=9 \& n=11$

33. Ratto, M., Cliveden, PB, Sparrowhawk KT. (2017). Improving learning and school achievement with an assessment and training program of basic cognitive function. Paper presented at the International Psychological Applications Conference and Trends 2017, Budapest, HU.

34. Sachdev, P. S., Blacker, D., Blazer, D. G., Ganguli, M., Jeste, D. V., Paulsen, J. S., \& Petersen, R. C. (2014). Classifying neurocognitive disorders: The DSM-5 approach. Nature Reviews Neurology, 10(11), 634-642. https://doi.org/10.1038/nrneurol.2014.181

35. Smith, P. J., Need, A. C., Cirulli, E. T., Chiba-Falek, O., \& Attix, D. K. (2013). A comparison of the Cambridge Automated Neuropsychological Test Battery (CANTAB) with "traditional" neuropsychological testing instruments. Journal of Clinical and Experimental Neuropsychology, 35(3), 319-328. https:// doi.org/10.1080/13803395.2013.771618

36. Spearman, C. (1904). "General intelligence", objectively determined and measured. American Journal of Psychology, 3, 271-295.

37. Tchanturia, K., Lounes, N., \& Holttum, S. (2014). Cognitive remediation in anorexia nervosa and related conditions: A systematic review. European Eating Disorders Review, 22(6), 454-462. https:// doi.org/10.1002/ erv.2326 
38. Thurstone, L. L. (1947). Multiple factor analysis. Chicago: University of Chicago Press.

39. olman, A. W., \& Kurtz, M. M. (2012). Neurocognitive predictors of objective and subjective quality of life in individuals with schizophrenia: A meta-analytic investigation. Schizophrenia Bulletin, 38(2), 304-315. https://doi.org/10.1093/schbul/sbq077

40. van de Weijer, S. C. F., Duits, A. A., Bloem, B. R., Kessels, R. P., Jansen, J. F. A., Köhler, S., ... Kuijf, M. L. (2016). The Parkin'Play study: Protocol of a phase II randomized controlled trial to assess the effects of a health game on cognition in Parkinson's disease. BMC Neurology, 16, 209. https://doi. org/10.1186/s12883-016-0731-z

41. van Hooren, S., Versmissen, D., Janssen, I., Myin-Germeys, I., A Campo, J., Mengelers, R., ... Krabbendam, L. (2008). Social cognition and neurocognition as independent domains in psychosis. Schizophrenia Research, 103(1-3), 257-265. https://doi.org/10.1016/j.schres. 2008.02.022

42. Wechsler, D. (2008). Wechsler adult intelligence scale (Fourth ed.). San Antonio, TX: Pearson.

43. Wykes, T., Huddy, V., Cellard, C., McGurk, S. R., \& Czobor, P. (2011). A meta-analysis of cognitive remediation for schizophrenia: Methodology and effect sizes. American Journal of Psychiatry, 168(5), 472-485. https://doi.org/10.1176/appi.ajp.2010.10060855 


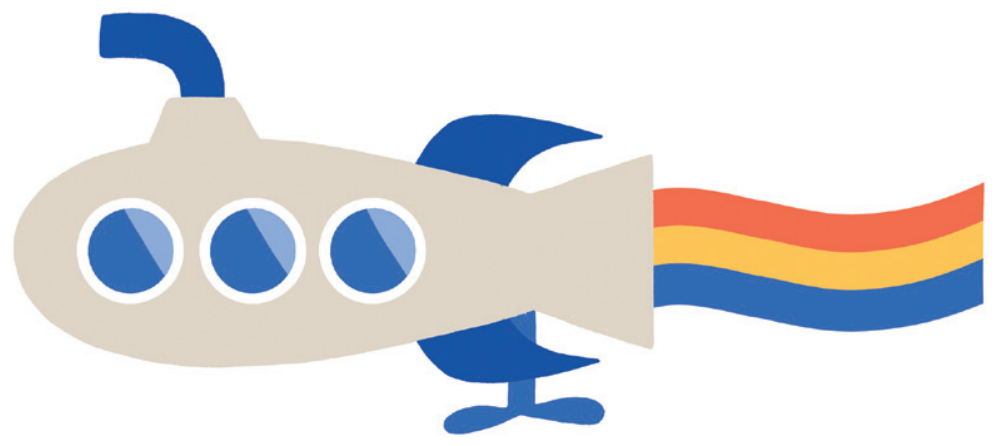




\section{CHAPTER 6}

The Parkin'Play study: protocol of a phase II randomized controlled trial to assess the effects of a health game on cognition in Parkinson's disease

Van de Weijer SCF, Duits AA, Bloem BR, Kessels RP, Jansen JFA, Köhler S, Tissingh G, Kuijf ML. BMC Neurol BioMed Central; 2016 Nov 3;16(1):209. PMID: 27809791 Parkinsonism and Related Disorders 2019 May;62:16-27. PMID: 30580907 


\section{ABSTRACT}

Background: In Parkinson's disease (PD), cognitive impairment is an important non-motor symptom heralding the development of dementia. Effective treatments to slow down the rate of cognitive decline in PD patients with mild cognitive impairment are lacking. Here, we describe the design of the Parkin'Play study, which assesses the effects of a cognitive health game intervention on cognition in PD.

Methods/Design: This study is a multicentre, phase-II, open-randomized clinical trial that aims to recruit 222 PD patients with mild cognitive impairment. Eligible patients have PD, Hoehn \& Yahr stages I-III, are aged between 40 and 75 years, and have cognitive impairment but no dementia. The intervention group $(n=111)$ will be trained using a web-based health game targeting multiple cognitive domains. The control group $(n=111)$ will be placed on a waiting list. In order to increase compliance the health game adapts to the subjects' performance, is enjoyable, and can be played at home. From each group, 20 patients will undergo fMRI to test for potential functional brain changes underlying treatment. The primary outcome after 12 weeks of training is cognitive function, as assessed by a standard neuropsychological assessment battery and an online cognitive assessment. The neuropsychological assessment battery covers the following domains: executive function, memory, visual perception, visuoconstruction and language. A compound score for overall cognitive function will be calculated as the mean score of all test Z-scores based on the distribution of scores for both groups taken together. Secondary outcomes at follow-up visits up to 24 weeks include various motor and non-motor symptoms, compliance, and biological endpoints (fMRI).

Discussion: This study aims at evaluating whether a cognitive intervention among PD patients leads to an increased cognitive performance on targeted domains. Strengths of this study are a unique web-based health game intervention, the large sample size, a control group without intervention and innovations designed to increase compliance. 


\section{BACKGROUND}

Non-motor symptoms in Parkinson's disease (PD) are now recognized as major contributors to a decreased quality of life $[1,2]$. Cognitive impairment is an important non-motor symptom and common in a substantial proportion of PD patients, even in early stages of the disease, and cognitive deficits typically worsen with disease progression [3]. Mild cognitive impairment in Parkinson's disease (or PD-MCI) is an umbrella term that refers to the heterogeneity of cognitive deficits in multiple domains. It describes the transition from healthy aging to dementia in which cognitive dysfunction is present, but no functional impairment [4]. PD is considered to be a fronto-striatal syndrome that gives rise to cognitive deficits that are particularly apparent when patients need to generate behaviour on the basis of internal rather than external cues, and when they need to flexibly switch between well-learned tasks [5]. Salient cognitive deficits in PD thus usually relate to deficits in attention and executive function, yet the overall cognitive profile is heterogeneous, with co-existing deficits in memory and visuospatial functions also being frequent.

Current treatment strategies for the cognitive deficits are partially effective at best. Even with optimal medical management, cognitive impairment remains a common and incapacitating problem for many PD patients. Therefore, adequate strategies to improve cognitive function and to possibly delay the onset of PD dementia are urgently needed.

The aging brain is thought to retain some degree of plasticity [6], which suggests that older adults may benefit from cognitive training programs. Most studies on the effect of cognitive training programs have been performed in healthy older adults, or in people with cognitive impairment due to vascular pathology or traumatic brain injury. Results showed that these programs could improve multiple domains of cognition [7-9]. So far, only a few cognitive intervention-studies have been conducted in PD patients [10-12]. Some have shown that cognitive training programs improved memory performance [11] and overall cognitive functions [12] after only six weeks of training. Furthermore, cognitive training resulted in a reduced risk of developing PD-MCl at a oneyear follow up assessment [12]. Indeed, a recent systematic review by Leung et al. [13] demonstrated a modest effect ( $g=0.23,95 \%$ confidence interval $0.014-0.44, p=0.037$ ) of cognitive training on cognitive function in patients with mild to moderate PD. According to Leung et al. [13], studies in larger samples are needed to examine the abilities of a cognitive training in preventing cognitive decline in PD.

The functional changes in the brain and mechanisms responsible for the associated degeneration process of $\mathrm{MCl}$ are unknown [14]. However, several changes in functional connectivity between selective brain regions also take place during this degeneration process. Visualization of these changes helps to localize the responsible underlying mechanisms and may be used as a tool for evaluation of future treatments. In PD-MCl, changes in global patterns of resting-state functional connectivity have been associated with widespread connectivity decrements in several networks, including the default-mode network (DMN) and occipital 
networks $[3,15,16]$. Most studies demonstrated a pronounced working-memory related under-recruitment of the striatum and dorsolateral prefrontal regions, but also increments of the connectivity of the DMN with posterior cortical regions. The under-recruitment of the striatum may be associated with a reduced capacity for working-memory updating through a decreased phasic release of dopamine [17], but direct evidence linking cognitive changes to underlying brain mechanisms in PD is sparse. A longitudinal study that followed PD patients for three years mainly found functional connectivity changes in the parietal, temporal, and occipital cortices that were associated with cognitive decline [18]. No longitudinal studies have been published on functional network activity changes in PD patients during active cognitive training.

Here, we describe the design of the Parkin'Play study, a multi-centre randomized controlled trial (RCT) that examines the effect of a web-based gaming service (MyCognition AquaSnap) on cognitive function in PD patients with cognitive impairment but no dementia. The gaming service includes both a cognitive training videogame (AquaSnap) and a cognitive assessment $\left(\mathrm{MyCQ}^{\mathrm{TM}}\right)$, which respectively trains and assesses the cognitive functions of a player on five core cognitive domains: attention, psychomotor speed, working memory, episodic memory, and executive function. The combination of these two components ensures an intervention adjusted to the (impaired) cognitive performance level of the subjects. Creating a web-based and adaptive health game that incorporates enhanced cognitive training loops may be more engaging, which may result in an increased compliance and effect in PD-MCl. The aim of this study is to determine whether a web-based gaming service designed for cognitive training is a feasible approach, and able to improve cognitive functioning within a three-month time frame in a new cohort of PD-MCI patients. We hypothesize that being able to train at home may optimize compliance to the intervention, as it motivates and rewards the patients.

\section{OBJECTIVES}

The primary objective of the Parkin'Play study is to evaluate whether an individually tailored multi-domain cognitive intervention (a health game) leads to an improvement in cognitive performance on various targeted domains, such as executive function, memory, visual perception, visuo-construction, and language. The secondary objective is to study whether the effects persists over time, after the intervention has ceased.

Additionally, given the limited number of studies among PD-patients, it is still unclear whether cognitive improvement due to cognitive training has a neurobiological basis. To provide us with evidence for a mechanistic explanation for the effect of the intervention and to locate the changes in various brain structures, we will assess task-based and resting-state fMRI. Specifically, we will analyse changes in connectivity patterns in the resting-state sensorimotor network, including the supplementary motor area, sensorimotor cortex, and secondary somatosensory cortex [19]. 


\section{METHODS/DESIGN}

\section{ETHICAL APPROVAL AND TRIAL REGISTRATION}

The study is carried out in compliance with the Helsinki Declaration. The local ethics committee of the Maastricht University Medical Centre has approved the study protocol, patient information letter, and the informed consent forms. Informed consent is obtained and signed by the patient prior to the screening session, after the patient is fully informed about the study and the procedures. The Parkin'Play study is registered in the Dutch trial registration under registration number NTR5637.

\section{STUDY DESIGN}

The Parkin'Play study is a multicenter phase-II openrandomized controlled study that aims to recruit 222 patients with Parkinson's disease with PD-MCl. Patients are randomly assigned to the intervention (cognitive training) or the control group (waiting list). All participants will undergo three assessments $(t=0, t=1$, and $t=2)$, which consist of neuropsychological assessments and questionnaires. In 20 patients from each group (total $n=40$ ) two fMRI scans will be obtained $(\mathrm{t}=0$ and $\mathrm{t}=1)$. The duration of the main intervention will be 12 weeks. In order to investigate the compliance and attractiveness of the health game, patients will be given the opportunity to continue playing for an additional 12 weeks (Fig. 1). The study will be analysed based on an intention-to-treat approach and results will be published on behalf of the Parkin'Play investigators.

\section{INTERVENTION}

The total duration of the intervention will be 12 weeks for both groups, with a voluntary extension of the cognitive training for an additional 12 weeks.

\section{COGNITIVE TRAINING (INTERVENTION GROUP)}

The intervention consists of a combination of two sofware products. To give people insight into their cognitive profile and tailor the intervention towards an individuals' weaknesses, the participants' cognitive functions will be assessed using an online assessment tool: MyCognition Quotient (MyCQ $\left.{ }^{\mathrm{TM}}\right)$. MyCQ ${ }^{\mathrm{TM}}$ is a cognitive assessment tool developed by the company MyCognition. Through ten individual tests, this assessment specifically evaluates the patients' capabilities in five core cognitive domains: attention, psychomotor speed, working memory, episodic memory, and executive function, consistent with other standard cognitive assessment targeted on Parkinson Disease [20, 21], and with the main neuropsychological domains normally considered as being affected by PD-MCI [22]. $\mathrm{MyCQ}^{\mathrm{TM}}$ is a 30-min assessment, which provides a personalized cognition score for each individual and a profile of strengths or weaknesses across the five core cognitive domains assessed. The individual tests that comprise the $\mathrm{MyCQ}^{\mathrm{TM}}$ assessment are based on validated paradigms and they are considered to be revised versions of paradigms that are commonly employed in the assessment of human cognition (see Table 1). The MyCQ ${ }^{\mathrm{TM}}$ was compared to the Cambridge Neuropsychological 
Automated Test Battery (CANTAB) in a population of fifty-five patients with psychiatric disorders [23]. Results indicated that most of the MyCQ ${ }^{\mathrm{TM}}$ subtests correlated with the CANTAB subtests of the corresponding domain.

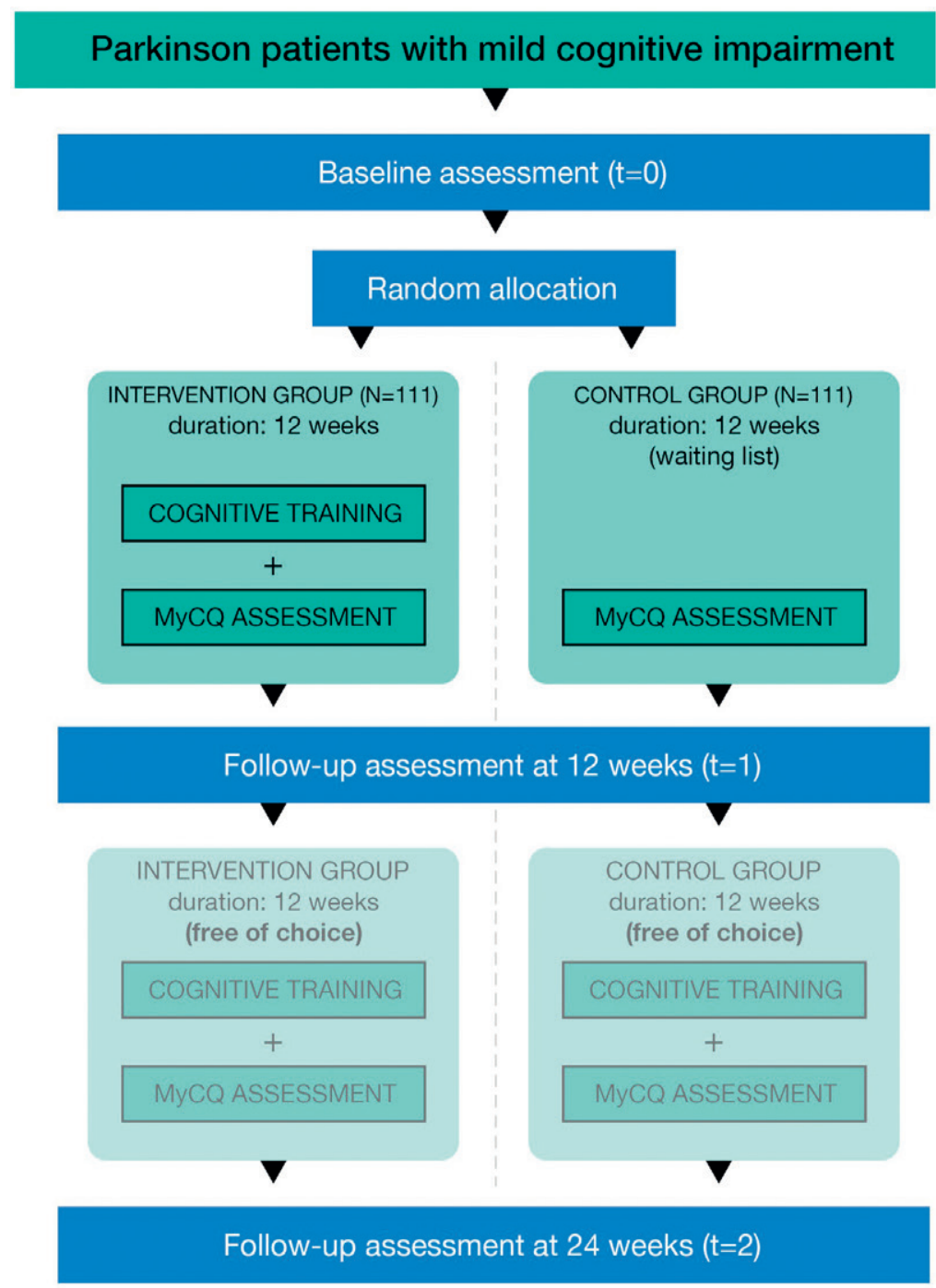

Figure 1. Design of the Parkin'Play study

The second product is a custom-made and web-based cognitive training AquaSnap that was built with input from experts of both Radboud University Medical Center and Maastricht University Medical Center. The adaptive cognitive training aims at exercising the cognitive domains of attention, working memory, episodic memory, psychomotor speed and executive function. In AquaSnap, played online on a PC/laptop or Apple iPad, a player is required to 
explore the ocean in an underwater rover and complete specific tasks by taking pictures of fish. The pictures are worth currency, which can be used to dive deeper into the sea to discover different aquatic environments with rarer fish. The $\mathrm{MyCQ}^{\mathrm{TM}}$ assessment is carried out monthly and according to an individuals' profile, AquaSnap adapts the speed and difficulty level of the game. The lower a player's MyCQ ${ }^{\mathrm{TM}}$ score, the more training tasks they need to complete in that specific domain. Each cognitive domain is mainly trained by a particular training loop, while some domains are trained across different tasks (see Table 2). The game develops on different structural levels. At the basic structural level there are the loops, which corresponds to the five first tasks in Table 2. The loops are organized in underwater dives, in which the player undergoes a set of loops. At the Ocean map level, users have to organise their dive in order to both achieve the proposed mission and to discover new areas. The progress of the players on the map, and consequently the growth of difficulty in the training game depend on the coins the players are able to collect during their dives. In this way the game adapts its difficulty to the level of progression reached by the player (Fig. 2). Additionally, as mentioned above, the intensity of the training depends on the individual $\mathrm{MyCQ}^{\mathrm{TM}}$ scores, as the number of loops for each type of task depends on the score obtained on each cognitive domain. In this way, more impaired domains will receive more intensive training.

Table 1. Individual $\mathrm{MyCQ}^{\mathrm{TM}}$ tests listed with test equivalents.

\begin{tabular}{|c|c|c|c|c|}
\hline & MycQ ${ }^{\mathrm{MM}}$ Test & $\begin{array}{l}\text { Cognitive domains } \\
\text { measures }\end{array}$ & $\begin{array}{l}\text { Paper \& pencil test } \\
\text { equivalent }\end{array}$ & $\begin{array}{l}\text { Computerised test } \\
\text { equivalent }\end{array}$ \\
\hline 1 & Simple Reaction Time & Psychomotor speed & Donders Type A & Detection \\
\hline 2 & Choice Reaction Time & Attention & Donders Type B & Identification \\
\hline 3 & $\mathrm{Go} / \mathrm{No} \mathrm{Go}^{\mathrm{a}}$ & Inhibition & Donders Type C & - \\
\hline 4 & $\begin{array}{l}\text { Verbal recognition } \\
\text { memory }\end{array}$ & Episodic memory & $\begin{array}{l}\text { Rey Auditory Verbal } \\
\text { Learning Test }\end{array}$ & $\begin{array}{l}\text { CDR Word } \\
\text { Recognition }\end{array}$ \\
\hline 5 & $\begin{array}{l}\text { Visual recognition } \\
\text { memory }\end{array}$ & Episodic memory & $\begin{array}{l}\text { Benton Visual } \\
\text { Retention Test }\end{array}$ & $\begin{array}{l}\text { CDR Picture } \\
\text { Recognition }\end{array}$ \\
\hline 6 & 1-Back ${ }^{\mathrm{a}}$ & Working memory & - & One Back \\
\hline 7 & 2-Back ${ }^{\mathrm{a}}$ & Working memory & - & Two Back \\
\hline 8 & Trail Making A & $\begin{array}{l}\text { Praxis/psychomotor } \\
\text { speed }\end{array}$ & $\begin{array}{l}\text { Trail Making Test } \\
\text { Part A }\end{array}$ & Chase Test \\
\hline 9 & Trail Making $\mathrm{B}^{\mathrm{a}}$ & $\begin{array}{l}\text { Praxis/psychomotor } \\
\text { speed/set shifting }\end{array}$ & $\begin{array}{l}\text { Trail Making Test } \\
\text { Part B }\end{array}$ & $\begin{array}{l}\text { Groton Maze } \\
\text { Learning Test }\end{array}$ \\
\hline 10 & Coding ${ }^{a}$ & $\begin{array}{l}\text { Psychomotor speed/ } \\
\text { attention }\end{array}$ & $\begin{array}{l}\text { Digit Symbol } \\
\text { Substitution Test }\end{array}$ & - \\
\hline
\end{tabular}

${ }^{\mathrm{a} A l s o}$ indexes elements of executive function

The duration of the cognitive training period is set at 12 weeks with a cumulative duration of 18 $\mathrm{h}$, divided over 36 sessions. In other cognitive training studies, the mean cumulative duration was $17,6 \mathrm{~h}$ with a range between 5 and $42 \mathrm{~h}$ and the mean training period was 9 weeks with a range between 4 and 24 weeks $[10,11,24-26]$. 
Time spent per day on gaming will be recorded automatically. There will no pre-set time limits in order to investigate the attractiveness of the games as well as the possibility of testing for addictive behaviour in post-hoc analyses. To prevent attrition due to an inadequate level of difficulty (i.e. too high or too low), coaches will contact the patients every two weeks in the first month to adjust the levels accordingly.

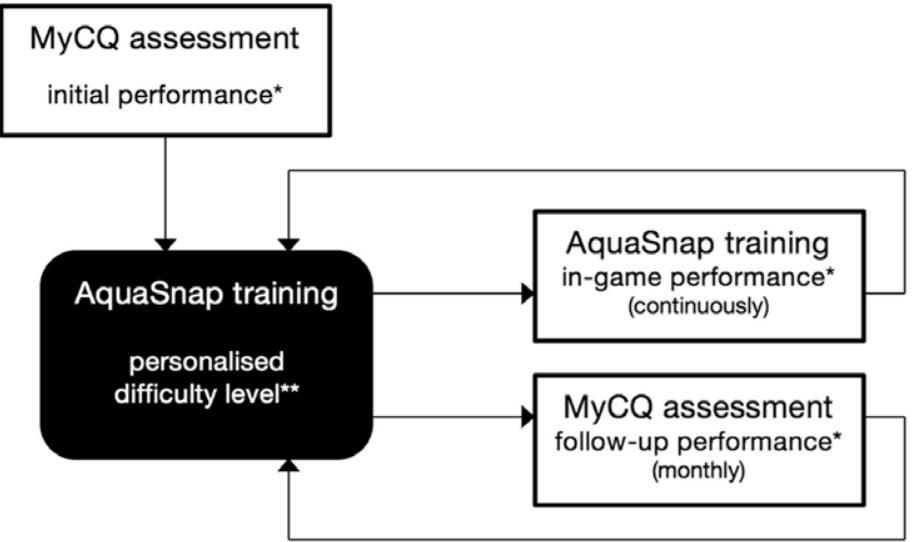

Figure 2. Feedback loops regulating the difficulty and pace of the AquaSnap training

\section{WAITING LIST (CONTROL GROUP)}

The control group is designed as a waiting list group in order to increase compliance. The participants are required to perform the $\mathrm{MyCQ}^{\mathrm{TM}}$ assessment monthly. For motivational purposes, the control group is offered to take part in a deferred intervention after 12 weeks (e.g. from week 12 until 24), with the same intensity as the intervention group in the first 12 weeks.

Table 2. Individual tasks in AquaSnap and trained domains

\begin{tabular}{llll}
\hline \multicolumn{1}{c}{ AquaSnap task } & Description & Cognitive domain \\
\hline 1 & Memory shot & $\begin{array}{l}\text { Remember the position of the glowing } \\
\text { fish in the loop }\end{array}$ & Working memory \\
\hline 2 & Quick shot & Snap the fish as soon as you see it & Processing speed and attention \\
\hline 3 & Careful quick shot & $\begin{array}{l}\text { Snap the fish as soon as you see it, but be } \\
\text { careful not to snap the shocking flash }\end{array}$ & $\begin{array}{l}\text { Attention and executive function } \\
\text { (inhibition) }\end{array}$ \\
\hline 4 & Group shot & Snap the group of fish all together & Attention and processing speed \\
\hline 5 & Fish Tracker & $\begin{array}{l}\text { Remember which fish are glowing after } \\
\text { they change position }\end{array}$ & Working memory \\
\hline 6 & Oceanic survey & $\begin{array}{l}\text { Remember which fish have you seen at } \\
\text { the end of the dive }\end{array}$ & Episodic memory \\
\hline 7 & $\begin{array}{l}\text { Missions and map } \\
\text { exploration }\end{array}$ & $\begin{array}{l}\text { Achieve the goals proposed by the daily } \\
\text { missions and try to discover new areas } \\
\text { on the ocean map }\end{array}$ & $\begin{array}{l}\text { Executive function (planning and } \\
\text { organizing) }\end{array}$ \\
\hline
\end{tabular}




\section{DROP-OUTS AND ADVERSE EVENTS}

Patients who drop out will be encouraged to complete the follow-up measurements. All unwanted and harmful outcomes spontaneously reported by the patients, that may or not be related to the treatment (adverse events), will be recorded according to section 10, subsection 1 of the Dutch Research Involving Human Subjects Act (WMO). Addiction to playing the game will be monitored by recording the amount of playing time and the BIS-11 scoring that measures changes in impulsivity. In case of a serious adverse event, the Ethics committee and relevant authorities (Toetsingonline and principal investigator) will be notified immediately.

\section{MEDICATION ADJUSTMENTS}

All participants are asked to keep their medication stable during the intervention period. Nonetheless, if medication changes are presumed necessary by the treating neurologist, they are allowed to do so. Any changes in medication will be noted in the case report form.

\section{STUDY POPULATION}

In- and exclusion criteria are shown in Table 3. Patients who are eligible for participating in the study have PD diagnosed by an experienced neurologist, with Hoehn and Yahr stages I-III, and are aged between 40 and 75 years. Furthermore, they have cognitive impairment but no dementia. Neuropsychological assessment including a global measure and a limited battery of standard tests will be used to assess whether $\mathrm{MCl}$ is present in accordance with the Level I criteria for the diagnosis of PD-MCI by Litvan et al. [27]. Patients must be on relatively stable dopaminergic medication for at least three months prior to inclusion, or the change in medication does not influence cognition. Patients should not receive any other cognitive therapy during the study period. Also, gamers who play any type of computer games more than one hour per week in the preceding year are excluded from participation.

Table 3. In- and exclusion criteria

\section{Inclusion criteria}

Idiopathic Parkinson's disease diagnosed by a neurologist

Hoehn \& Yahr stage $\leq 3$

Age 40-75 years

Mild cognitive impairment according to MDS

(MCl Level 1 criteria)

Relatively stable dopaminergic medication dose for at least three months prior to the study, or change in medication does not influence cognition. Deemed unlikely to start or stop treatment within the next three months.

\section{Exclusion criteria}

Medication affecting cognition (such as anticholinergic drugs, benzodiazepine and methylfenidate) Other medical conditions:

- Advanced problems in cognitive functioning (MoCA <19/30)

- Dementia

- Active depression or psychosis and/or treatment with anti- depressant or antipsychotic drugs

- History of active thyroid disease, stroke with residual deficits, severe hypertension or diabetes or head trauma interfering in cognition Severe auditory of visual deficits

No internet at home

fMRI sub-study:

- Metal in the body

- Claustrophobia 


\section{RECRUITMENT AND SETTING}

The treating neurologist will evaluate eligibility of patients using a checklist. After eligible patients have been informed about the study and have agreed to participate, they will be invited for a screening assessment. Informed consent is signed prior to the screening assessment. The inclusion period for the Parkin'Play study will last 18 months. In order to reach target sample size, neurologists from Maastricht University Medical Center, Radboud University Medical Center Nijmegen, and Zuyderland Medical Center Heerlen will be involved in recruitment. Participants are currently being recruited and enrolled. For practical reasons, only patients from Maastricht University Medical Center will be asked to join the fMRI substudy, prior to the screening assessment. We stop including fMRI participants when the goal of 20 patients per arm is reached.

\section{RANDOMIZATION AND BLINDING}

After the baseline measurements are performed, participants will be randomly assigned to one of two groups: the Intervention group (IG), or the Control group placed on a waiting list (CG), in a 1:1 ratio. Randomisation (minimisation) will be performed by the Clinical Trial Centre Maastricht (not formally involved in the study or assessments) using the software package ALEA (Formsvision BV). Stratification factors include site location (Maastricht, Nijmegen, or Heerlen) and age group ( $<60$ and $>=60$ years old). The study is an open- randomised controlled trial, so there will be no blinding for treatment allocation. Nonetheless, all follow-up measurements will be assessed by blinded outcome assessors. Patients will be enrolled by a coordinating investigator, who assigns patients to the intervention groups.

\section{OUTCOME MEASURES}

The primary outcome is global cognitive function, as assessed by both a standard neuropsychological assessment and the $\mathrm{MyCQ}^{\mathrm{TM}}$, at baseline and 12 weeks after baseline assessment. The standard neuropsychological assessment consists of various tests covering the following domains: executive function (Stroop Colour Word Test [28], category fluency and letter fluency in parallel versions [29]), memory (Rey Auditory Verbal Learning Test in parallel versions [30], Location Learning Test [31]), visual perception (Judgement of Line Orientation in parallel versions [32]), visuoconstruction (Rey-Osterrieth Complex Figure [33, 34]), and language (Boston Naming Test - Short Form). A compound score for overall (global) cognitive function will be calculated as the mean score of all test Z-scores based on the distribution of scores for both groups taken together. Z-scores of tests with higher scores representing worse performance will be inverted before computing the compound scores. By comparing Z-scores across different neuropsychological tests, the neuropsychological profiles of participants can be directly compared to identify the domains that benefitted most from the intervention.

The participants will also perform a monthly web-based online neuropsychological assessment $\left(\mathrm{MyCQ}^{\mathrm{TM}}\right)$, which is part of the intervention program. Since the MyCQ ${ }^{\mathrm{TM}}$ is integrated in the AquaSnap health game (i.e., the difficulty level of the game automatically adapts according 
to $\mathrm{MyCQ}{ }^{\mathrm{TM}}$ performance), it is not an independent measure of treatment effects, and hence the standard neuropsychological test battery is the primary outcome measure.

\section{ADDITIONAL STUDY PARAMETERS}

\section{Non-motor symptoms}

Several assessments will be performed at baseline. The Montreal Cognitive Assessment (MoCA) is used to assess global cognition and it is suggested to be more sensitive to cognitive impairment in PD populations in comparison with the Mini Mental State Exam (MMSE) [35]. MoCA includes several neuropsychological items focussing on memory, language, executive function, and visuospatial processing [36]. The Dutch National Adult Reading Task (NART) estimates premorbid intelligence levels. The test is untimed and consists of 50 words with atypical phonemic pronunciation. Each word is presented individually and subjects are asked to read them out loud [37].

Depression will be assessed with the Hamilton Anxiety and Depression Scale (HADS) [38], which has been validated in PD [39]. The Epworth Sleepiness Scale is used to provide a measurement of the subject's general level of daytime sleepiness [40]. The Cognitive Failure Questionnaire (CFQ) contains 25 questions and provides a self-report evaluation of perception, memory and motor-function in daily life [41]. The Parkinson's Disease Cognitive Functional Rating Scale (PD-CFR) is a 12-item questionnaire for rating functional abnormalities associated to cognitive impairment in non-demented PD patients [42]. The pre-Rasch-built Overall Disability Scale (pre-R-ODS) can be used to capture functional disability of the patients in a descriptive way [43]. Quality of life will be assessed with the Parkinson Disease Questionnaire (PDQ-39) [44]. The Barratt Impulsiveness Scale 11 (BIS-11) is a 30-item self-report questionnaire that measures impulsive behaviour [45].

\section{Motor functioning}

The unified Parkinson's disease rating scale (MDS-UPDRS) part III is used to assess and monitor disability and impairment in PD patients.

\section{Compliance}

Compliance and attractiveness of the health game will be determined based on the number of dropouts and the cognitive training duration in both groups. Additionally, extended compliance in the intervention group, who continue to play the health game between 12 and 24 weeks from baseline, will be tracked. Comparable studies among both healthy subjects as well as PD patients have found increased cognitive abilities after 6 months follow-up [46, 47] and 1 year follow-up [12]. Based on these studies, we expect to find long term benefits of AquaSnap. Moreover, with a 24 week follow-up measurement, we're able to test the feasibility of the intervention based on the motivation of the subjects to continue playing AquaSnap. 
fMRI

MRI imaging will be performed on a 3.0-Tesla unit and in the ON-medication state. Imaging will consist of a neuroradiological protocol (T1-weighted sequences), supplemented with full brain functional imaging ( $\mathrm{N}$-back fMRI and resting-state fMRI for functional connectivity imaging). T1 scans will be used to assess macro-structural findings. Volumetric assessment of T1-weighted images will be performed using FreeSurfer software. Task based fMRI will be assessed using SPM and resting state fMRI will be assessed using Melodic. In addition, changes in the resting-state sensorimotor network, including the supplementary motor area, sensorimotor cortex, and secondary somatosensory cortex [19], will be analysed using a robust data-driven approach: independent component analysis (ICA) [48, 49]. Imaging will be performed at the Department of Radiology of the Maastricht University Medical Center. The assessors performing the fMRI baseline and follow-up assessments are blinded for treatment allocation.

\section{STUDY ACTIVITIES}

Enrolment will take place in the weeks prior to baseline assessment, except for the additional inclusion assessments (see Table 4). The following demographics and general variables are collected at baseline: date of birth, sex, educational level, age at onset, disease duration, and details on Parkinson medication (i.e., drug name, dose, frequency, levodopa equivalence). Details on Parkinson medication will also be collected at both follow-up measurements. Randomization will be performed after the baseline assessment. Primary and additional outcome assessments will be performed at baseline, after 12 and after 24 weeks follow up. The additional fMRI scans will be performed at baseline and after 12 weeks, matching the primary outcome assessments. The intervention group will be required to play the health game from start of the intervention until week 12 for at least 36 sessions of 30 min (three times a week). After week 12, both the intervention as well as the control group will be allowed to continue playing the health game free of choice. All patients will be examined in their on-state.

\section{DATA COLLECTION AND MANAGEMENT}

All personnel involved in data collection will review the standard operating procedures (SOP) and manuals. Assessors will be certified in Good Clinical Practice (GCP), certified in performing the MDS-UPDRS-III, and trained in assessing the neuropsychological test battery and other assessments by experienced raters. Data will be collected on paper forms and entered into a web-based data entry portal. Questionnaires are completed digitally and are imported automatically in the electronic database, of which a backup will be made daily. A member of the research team will monitor inclusion progress and data collection progress. After completion of the Parkin'Play study, the database will be approved and locked before data-analysis is set in motion. 
Table 4. Study schedule and assessments

\begin{tabular}{|c|c|c|c|c|c|c|c|}
\hline & Timepoint $\rightarrow$ & $-t 1$ & $t 0$ & & & $t 1$ & $t 2$ \\
\hline & Visit \# $\rightarrow$ & 0 & 1 & & & 2 & 3 \\
\hline & Week \# $\rightarrow$ & -1 to -3 & 0 & 4 & 8 & 12 & 24 \\
\hline \multirow[t]{8}{*}{ Enrolment } & \multicolumn{7}{|l|}{ Screening \& inclusion assessments } \\
\hline & Regular clinical intake & $x$ & & & & & \\
\hline & Demographics & $x$ & & & & & \\
\hline & Inclusion/exclusion & $x$ & & & & & \\
\hline & National Adult Reading Task & & $x$ & & & & \\
\hline & Montreal Cognitive Assessment & & $x$ & & & & \\
\hline & Epworth Sleepiness & & $x$ & & & & \\
\hline & Levodopa equivalent dose (LED) & & $x$ & & & $x$ & $x$ \\
\hline \multirow[t]{10}{*}{ Assessments } & \multicolumn{7}{|l|}{ Primary outcomes } \\
\hline & Standard neuropsychological battery & & $x$ & & & $x$ & $x$ \\
\hline & $\mathrm{MyCQ}^{\mathrm{TM}}$ assessment & & $x$ & $x$ & $\mathrm{X}$ & $x$ & $x$ \\
\hline & \multicolumn{7}{|l|}{ Additional outcomes } \\
\hline & Motor function (UPDRS part III) & & $x$ & & & $x$ & $x$ \\
\hline & Self-report questionnaires & & $x$ & & & $x$ & $x$ \\
\hline & Parkinson Disease Questionnaire-39 & & $\mathrm{X}$ & & & $x$ & $x$ \\
\hline & Pre-Rasch-built Overall Disability Scale & & $\mathrm{X}$ & & & $x$ & $x$ \\
\hline & fMRI exclusion criteria & $x$ & & & & & \\
\hline & fMRI scan ${ }^{a}$ & & $x$ & & & $x$ & \\
\hline \multirow[t]{2}{*}{ Interventions } & Randomization & & $x$ & & & & \\
\hline & Game playing ( 36 sessions of $30 \mathrm{~min}$ ) & & $x$ & $x$ & $x$ & $x$ & $x$ \\
\hline
\end{tabular}

a Only for a subset of 40 subjects.

\section{STATISTICAL ANALYSIS PROCEDURE}

Differences between the intervention and control group in global cognitive function (compound score of the neuropsychological test battery) at 12 weeks (primary endpoint) will be compared using independent sample t-tests. In addition, differences between groups in the individual cognitive domains will be tested using multivariate analysis of variance (MANOVA). Change in cognition over time (baseline, 12 weeks, 24 weeks) will be tested using repeated-measures ANOVA. All tests will be two-tailed with alpha set at 0.05 . The assumptions of normality and homogeneity of variance will be assessed by inspection of normal probability plots and residual plots. In case assumptions are not met, appropriate data transformations will be used. Secondary outcomes will be presented as means, standard deviations, minimum, maximum, median, lower and upper quartiles. The number of observations and changes from baseline will be presented. Categorical data will be presented in contingency tables as frequencies and 
percentages. The analyses will be performed on an intention-to-treat basis. A secondary "PerProtocol" analysis will also be included for the patients who have fully completed the study protocol for the primary endpoint. In order to investigate the effect of 'missingness', sensitivity analyses will be performed, including missing data augmentation using multiple imputation by chained equations [45] and maximum likelihood estimation with random effects.

For the main study, interim analysis will be conducted after 40 patients have completed the 12-week cognitive training period, including the corresponding assessments, to find out if the training is particularly beneficial or not. If, after interim analysis, it can be concluded that the training is particularly harmful, we may end the study and analyze the study data. This will only be decided in consultation with the local ethics committee.

\section{POWER AND SAMPLE SIZE ESTIMATE}

The Parkin'Play study is powered to show a moderate effect of the AquaSnap cognitive training on the standard neuropsychological assessment. A study in healthy older participants compared a brain training program (Brain Age) to a control condition (Tetris gaming) and found an ANCOVA-based effect size of Cohen's $f=0.39$ (eta2 $=0.13$ ) on the Trail Making Test, which assesses cognitive flexibility, often reduced in PD [9]. This reflects a moderate-to-large effect size (equivalent of Cohen's d of 0.78 ). The present population includes participants aged between 40 and 75 years old, but with PD. Still, we expect a moderate effect size (0.4-0.5). Therefore, the estimated sample size for detecting a difference between the means of the treatment groups on the compound score of standard neuropsychological tests, with a power of 0.8 and a Cohen's $d=0.4$, at an alpha-level of 0.05 , for a two-sided test, and an allocation ratio of $1: 1$ is 200 patients.

Based on previous cognition training and video game clinical trials the attrition rate is estimated at $10 \%$, which yields an additional 22 patients that should be recruited for participation $[9,11,50]$. This leaves a total of 222 patients that should be recruited.

\section{DISCUSSION}

The overall aim of this phase-2 multi-center open-randomized controlled clinical trial among PD patients is to evaluate whether a web-based health game designed to train cognition leads to an improvement in cognitive performance on targeted cognitive domains, relative to a control group on a waiting list. The strengths of the Parkin'Play study are the large sample size $(n=222)$, the gamified and home-based training approach, the individually tailored and adaptive training, and the application of $\mathrm{fMRI}$ assessments in order to explore underlying brain mechanisms. The addition of the 24-week follow up assessment provides valuable information about maintenance of benefit and about the feasibility of implementing health games in a PD population. 
Now that the new MDS-criteria for PD-MCl have been introduced, this study will for the first time study a large sample of patients fulfilling the proposed criteria in a clinical trial [13]. Previous cognitive clinical trials for PD have incorporated a range of cognitive and functional outcome measures, partly based on scales derived from Alzheimer's disease [51, 52]. This illustrates the lack of consensus on cognitive outcome measures. Furthermore, regulatory agencies may require measures that take into account a functional benefit for the patient. In this study, a compound score from a neuropsychological test battery will be used and complemented with the MyCQ ${ }^{\mathrm{TM}}$. The recently validated PD-CFRS scale was chosen to study functional benefit with demonstrated responsiveness over time [53].

The Parkin'Play intervention consists of various innovations. Firstly, performing cognitive training at home is likely to optimize compliance, since PD patients' reduced mobility might prevent them from attending outdoor activities $[12,47]$. Secondly, a task that is too hard may result in anxiety and therefore lead to resistance of collaboration to the treatment (e.g. stop playing the health game). The health game and $\mathrm{MyCQ}^{\mathrm{TM}}$ assessment combination adapts to the subject's capabilities and thereby results in a challenging game specifically tailored to the individual. Thirdly, the health game aims at being fun to play, introducing a multi-layer game story opposed to abstract repetitive cognitive brain trainings. These innovations are aimed at increasing the compliance of the intervention and prevent substantial attrition rates associated with study failures.

Cognitive training is thought to have beneficial effects on cognitionthrough activating mechanisms of the brain plasticity. Brain plasticity refers to the capacity of the central nervous system to change or to adapt its structure and function over a lifetime [54,55]. There is evidence that training on demanding tasks (adaptive training) decreases activation in frontal, parietal, and occipital regions of older adults, which may reflect improved neural efficiency and reduced use of resources [56]. Cognitive training has proved to be able to produce improvement in the main neuropsychological domains involved in PD as memory, attention, processing speed and executive function. Specific studies on the effects of cognitive training on PD have recently been conducted [26]. The results of randomized control trials show that cognitive and affective functions can be improved by cognitive trainings in PD patients. To our knowledge, no cognitive videogame training interventions have been conducted in PD-MDI.

Mild cognitive impairment in PD is generally viewed as a pre-dementia stage in PD, but the functional changes in the brain and mechanisms responsible for the associated degeneration process are unknown. Since the aged brain still retains neuroplasticity [6], it may restore or prevent functional changes of brain network activity. Indeed, in a non-imaging study, Petrelli et al. [12] have found that patients in the cognitive training intervention group had a reduced risk of developing $\mathrm{MCl}$ after 1 year follow up. Nonetheless, no longitudinal studies have been published on functional changes in PD patients during cognitive training. With the use of fMRIimaging, we hope to have a better understanding on functional network activity changes in $\mathrm{PD}$ patients as a result of active cognitive training. Visualization of these changes helps to 
localize the responsible underlying mechanisms and may be used as a tool for evaluation of future treatments.

The Parkin'Play study contributes to a better understanding of cognitive impairment in PD and evaluates a new possible non-pharmacological intervention for PD-MCI.

\section{ACKNOWLEDGEMENTS}

Not applicable.

\section{FUNDING}

The Parkin'Play study is funded by MyCognition, London, UK. The sponsors had no role in the design of the study and collection, analysis, and interpretation of data, and in writing the manuscript.

\section{AVAILABILITY OF DATA AND MATERIALS}

Datasets will be made available from the corresponding author on reasonable request.

\section{AUTHORS' CONTRIBUTIONS}

$B B$ initiated the study. MK, BB, and AD drafted the first version of the protocol. SW and MK initiated the study design and assisted in the design of the health game. BB, MK, GT, and AD provided expertise in trial design. SK contributed to the statistical paragraphs. JJ contributed to the design of the fMRI sub-study. All authors contributed to the study protocol and approved the final manuscript.

\section{COMPETING INTERESTS}

The authors declare to have no competing interests.

\section{CONSENT FOR PUBLICATION}

Not applicable.

\section{ETHICS APPROVAL AND CONSENT TO PARTICIPATE}

The local ethics committee of the Maastricht University Medical Centre has approved the study protocol, patient information letter, and the informed consent forms. The committee's reference number is 141128 . 


\section{REFERENCES}

1. Leroi I, McDonald K, Pantula H, Harbishettar V. Cognitive impairment in Parkinson disease: impact on quality of life, disability, and caregiver burden. J Geriatr Psychiatry Neurol. 2012;25(4):208-14. SAGE Publications.

2. Lawson RA, Yarnall AJ, Duncan GW, Khoo TK, Breen DP, Barker RA, et al. Severity of mild cognitive impairment in early Parkinson's disease contributes to poorer quality of life. Parkinsonism Relat Disord. 2014;20(10):1071-5.

3. Yarnall AJ, Breen DP, Duncan GW, Khoo TK, Coleman SY, Firbank MJ, et al. Characterizing mild cognitive impairment in incident Parkinson disease The ICICLE-PD Study. Neurology. 2014;82(4):308-16. AAN Enterprises.

4. Petersen RC. Mild cognitive impairment as a diagnostic entity. J Intern Med. 2004;256(3):183-94.

5. Barker RA, Williams-Gray CH. Mild Cognitive Impairment and Parkinson's Disease-Something to Remember. J Parkinsons Dis. 2014;4(4):651-6. IOS Press.

6. Jones S, Nyberg L, Sandblom J, Stigsdotter-Neely A, Ingvar M, Magnus Petersson K, et al. Cognitive and neural plasticity in aging: general and task-specific limitations. Neurosci Biobehav Rev. 2006;30(6):864-71.

7. Ngandu T, Lehtisalo J, Solomon A, Levälahti E, Ahtiluoto S, Antikainen R, et al. A 2 year multidomain intervention of diet, exercise, cognitive training, and vascular risk monitoring versus control to prevent cognitive decline in at-risk elderly people (FINGER): a randomised controlled trial. Lancet. 2015.

8. Anguera JA, Boccanfuso J, Rintoul JL, Al-Hashimi O, Faraji F, Janowich J, et al. Video game training enhances cognitive control in older adults. Nature. 2013;501(7465):97-101.

9. Nouchi R, Taki Y, Takeuchi H, Hashizume H, Akitsuki Y, Shigemune Y, et al. Brain Training Game Improves Executive Functions and Processing Speed in the Elderly: A Randomized Controlled Trial. Aleman A, editor. PLoS One. 2012;7(1):e29676.

10. Sammer G, Reuter I, Hullmann K, Kaps M, Vaitl D. Training of executive functions in Parkinson's disease. J Neurol Sci. 2006;248(1-2):115-9.

11. Naismith SL, Mowszowski L, Diamond K, Lewis SJG. Improving memory in Parkinson's disease: A healthy brain ageing cognitive training program. Mov Disord. 2013;28(8):1097-103.

12. Petrelli A, Kaesberg S, Barbe MT, Timmermann L, Rosen JB, Fink GR, et al. Cognitive training in Parkinson's disease reduces cognitive decline in the long term. Eur J Neurol. 2015;22(4):640-7.

13. Leung IHK, Walton CC, Hallock H, Lewis SJG, Valenzuela M, Lampit A. Cognitive training in Parkinson disease: A systematic review and meta-analysis. Neurology. 2015;30.

14. Chaudhuri KR, Schapira AHV. Non-motor symptoms of Parkinson's disease: dopaminergic pathophysiology and treatment. Lancet Neurol. 2009;8(5): 464-74. 
15. Garcia-Diaz AI, Segura B, Baggio HC, Marti MJ, Valldeoriola F, Compta Y, et al. Parkinsonism and Related Disorders. Parkinsonism Relat Disord. 2014; 20(12):1405-10. Elsevier Ltd.

16. Nagano-Saito A, Habak C, Mejía-Constaín B, Degroot C, Monetta L, Jubault T, et al. Neurobiology of Aging. Neurobiol Aging. 2014;35(1):223-31. Elsevier Ltd.

17. Ekman U, Eriksson J, Forsgren L, Jakobson Mo S, Riklund K, Nyberg L. Functional brain activity and presynaptic dopamine uptake in patients with Parkinson's disease and mild cognitive impairment: a cross-sectional study. Lancet Neurol. 2012;11(8):679-87. Elsevier Ltd.

18. Dubbelink KTEO, Schoonheim PMM, Deijen PJB, Twisk PJWR, Barkhof PF, Berendse PHW. Functional connectivity and cognitive decline over 3 years in Parkinson disease. Neurology. 2014;2014(83):204753.

19. Smith SM, Fox PT, Miller KL, Glahn DC, Fox PM, Mackay CE, et al. Correspondence of the brain's functional architecture during activation and rest. Proceedings of the National Academy of Sciences of the United States of America. National Acad Sci. 2009;106(31):13040-5.

20. Dalrymple-Alford JC, MacAskill MR, Nakas CT, Livingston L, Graham C, Crucian GP, et al. The MoCA: Well-suited screen for cognitive impairment in Parkinson disease. Neurology. 2010;75(19):1717-25. Lippincott Williams \& Wilkins.

21. Pagonabarraga J, Kulisevsky J, Llebaria G, García-Sánchez C, Pascual-Sedano B, Gironell A. Parkinson's disease-cognitive rating scale: a new cognitive scale specific for Parkinson's disease. Mov Disord. 2008;23(7):998-1005.

22. Goldman JG, Holden S, Ouyang B, Bernard B, Goetz CG, Stebbins GT. Diagnosing PD-MCI by MDS task force criteria: How many and which neuropsychological tests? Mov Disord. 2015;30(3):402-6.

23. Domen AC, Kumar R, Harrison J, De Haan L, Denys DAJP, Nieman DH. The validation of a new online cognitive assessment tool. ECNP Conference. 2016. https://www.ecnp.eu/presentationpdfs/57/P.1.j.037.pdf.

24. Nombela C, Bustillo PJ, Castell PF, Sanchez L, Medina V, Herrero MT. Cognitive Rehabilitation in Parkinson's Disease: Evidence from Neuroimaging. Front Neurol. 2011;2.

25. Zimmermann R, Gschwandtner U, Benz N, Hatz F, Schindler C, Taub E, et al. Cognitive training in Parkinson disease Cognition-specific vs nonspecific computer training. Neurology. 2014;82(14):1219 26. AAN Enterprises.

26. Petrelli A, Kaesberg S, Barbe MT, Timmermann L, Fink GR, Kessler J, et al. Effects of cognitive training in Parkinson's disease: a randomized controlled trial. Parkinsonism Relat Disord. 2014;20(11):1196-202.

27. Litvan I, Goldman JG, Tröster AI, Schmand BA, Weintraub D, Petersen RC, et al. Diagnostic criteria for mild cognitive impairment in Parkinson's disease: Movement Disorder Society Task Force guidelines. Mov Disord. 2012;27(3): 349-56.

28. Jensen AR, Rohwer WD. The Stroop color-word test: a review. Acta Psychol (Amst). 1966;25(1):36-93.

29. Schmand B, Groenink SC, van den Dungen M. Letter fluency: psychometric properties and Dutch normative data. Tijdschr Gerontol Geriatr. 2008;39(2):64-76. 
30. Vakil E, Blachstein H. Rey Auditory-Verbal Learning Test: structure analysis. J Clin Psychol. 1993;49(6):883-90.

31. Kessels RPC, Nys GMS, Brands AMA, van Zandvoort MJE. The Location Learning Test as a measure of spatial memory: applicability of a modified administration procedure and normative data. Tijdschr Gerontol Geriatr. 2004;35(4):147-52.

32. Treccani B, Torri T, Cubelli R. Is judgement of line orientation selectively impaired in right brain damaged patients? Neuropsychologia. 2005; 43(4):598-608.

33. Rey A. L'examen psychologique dans les cas d'encéphalopathie traumatique. (Les problems.). Archives de Psychologie. 1941;28:215-85.

34. Osterrieth PA. Le test de copie d'une figure complexe; contribution à l'étude de la perception et de la mémoire. Archives de Psychologie. 1944; 30:206-56.

35. Zadikoff C, Fox SH, Tang-Wai DF, Thomsen T, de Bie RMA, Wadia P, et al. A comparison of the mini mental state exam to the Montreal cognitive assessment in identifying cognitive deficits in Parkinson's disease. Mov Disord. 2008;23(2):297-9. Wiley Subscription Services, Inc., A Wiley Company.

36. Kandiah N, Zhang A, Cenina AR, Au WL, Nadkarni N, Tan LC. Montreal Cognitive Assessment for the screening and prediction of cognitive decline in early Parkinson's disease. Parkinsonism Relat Disord. 2014;20(11):1145-8.

37. Schmand B, Bakker D, Saan R, Louman J. The Dutch Reading Test for Adults: a measure of premorbid intelligence level. Tijdschr Gerontol Geriatr. 1991;22(1):15-9.

38. Zigmond AS, Snaith P. The hospital anxiety and depression scale. 1983. 1 p.

39. Marinus J, Leentjens AFG, Visser M, Stiggelbout AM, van Hilten JJ. Evaluation of the hospital anxiety and depression scale in patients with Parkinson's disease. Clin Neuropharmacol. 2002;25(6):318-24.

40. Johns MW. A new method for measuring daytime sleepiness: the Epworth sleepiness scale. sleep. 1991.

41. Broadbent DE, Cooper PF, FitzGerald P, Parkes KR. The Cognitive Failures Questionnaire (CFQ) and its correlates. Br J Clin Psychol. 2011;21(1):1-16. Blackwell Publishing Ltd.

42. Kulisevsky J, de Bobadilla RF, Pagonabarraga J, Martínez-Horta S, Campolongo A, García-Sánchez C, et al. Parkinsonism and Related Disorders. Parkinsonism Relat Disord. 2013;19(9):812-7. Elsevier Ltd.

43. van Nes SI, Vanhoutte EK, Van Doorn PA, Hermans M, Bakkers M, Kuitwaard K, et al. Rasch-built Overall Disability Scale (R-ODS) for immune-mediated peripheral neuropathies. Neurology. 2011;76(4):337-45. AAN Enterprises.

44. Jenkinson C, Fitzpatrick R, Peto V, Greenhall R, Hyman N. The Parkinson's Disease Questionnaire (PDQ-39): development and validation of a Parkinson's disease summary index score. Age and Ageing. Br Geriatrics Soc; 1997;26(5):353-7

45. Patton JH, Stanford MS, Barratt ES. Factor structure of the Barratt impulsiveness scale. J Clin Psychol. 1995;51:768-74. 
46. Ball K, Berch DB, Helmers KF, Jobe JB, Leveck MD, Marsiske M, et al. Effects of cognitive training interventions with older adults: a randomized controlled trial. JAMA. 2002;288(18):2271-81.

47. Smith GE, Housen P, Yaffe K, Ruff R, Kennison RF, Mahncke HW, et al. A cognitive training program based on principles of brain plasticity: results from the Improvement in Memory with Plasticity-based Adaptive Cognitive Training (IMPACT) study. J Am Geriatr Soc. 2009;57(4):594-603.

48. Beckmann CF, DeLuca M, Devlin JT, Smith SM. Investigations into resting-state connectivity using independent component analysis. Philos Trans Roy Soc B. 2005;360(1457):1001-13.

49. Calhoun VD, Liu J, Adalı T. A review of group ICA for fMRI data and ICA for joint inference of imaging, genetic, and ERP data. Neurolmage. 2009;45(1):S163-72.

50. París AP, Saleta HG, la Cruz Crespo Maraver de M, Silvestre E, Freixa MG, Torrellas CP, et al. Blind randomized controlled study of the efficacy of cognitive training in Parkinson's disease. Mov Disord. 2011;26(7):1251-8.

51. Emre M, Aarsland D, Albanese A. Rivastigmine for dementia associated with Parkinson's disease. N Engl J Med. 2004;351(24):2509-18.

52. Dubois B, Tolosa E, Katzenschlager R, Emre M, Lees AJ, Schumann G, et al. Donepezil in Parkinson's disease dementia: A randomized, double-blind efficacy and safety study. Mov Disord. 2012;27(10):1230-8. Wiley Subscription Services, Inc., A Wiley Company.

53. Fernández de Bobadilla R, Pagonabarraga J, Martínez-Horta S, Pascual-Sedano B, Campolongo A, Kulisevsky J. Parkinson's disease-cognitive rating scale: Psychometrics for mild cognitive impairment. Mov Disord. 2013;28(10):1376-83.

54. Freitas C, Farzan F, Pascual-Leone A. Assessing brain plasticity across the lifespan with transcranial magnetic stimulation: why, how, and what is the ultimate goal? Frontiers in neuroscience. Frontiers. 2013;7:42.

55. Sehgal M, Song C, Ehlers VL, Moyer JR. Learning to learn intrinsic plasticity as a metaplasticity mechanism for memory formation. Neurobiol Learn Mem. 2013;105:186-99.

56. Brehmer Y, Rieckmann A, Bellander M, Westerberg H, Fischer H, Bäckman L. Neural correlates of training-related working-memory gains in old age. Neurolmage. 2011;58(4):1110-20. 


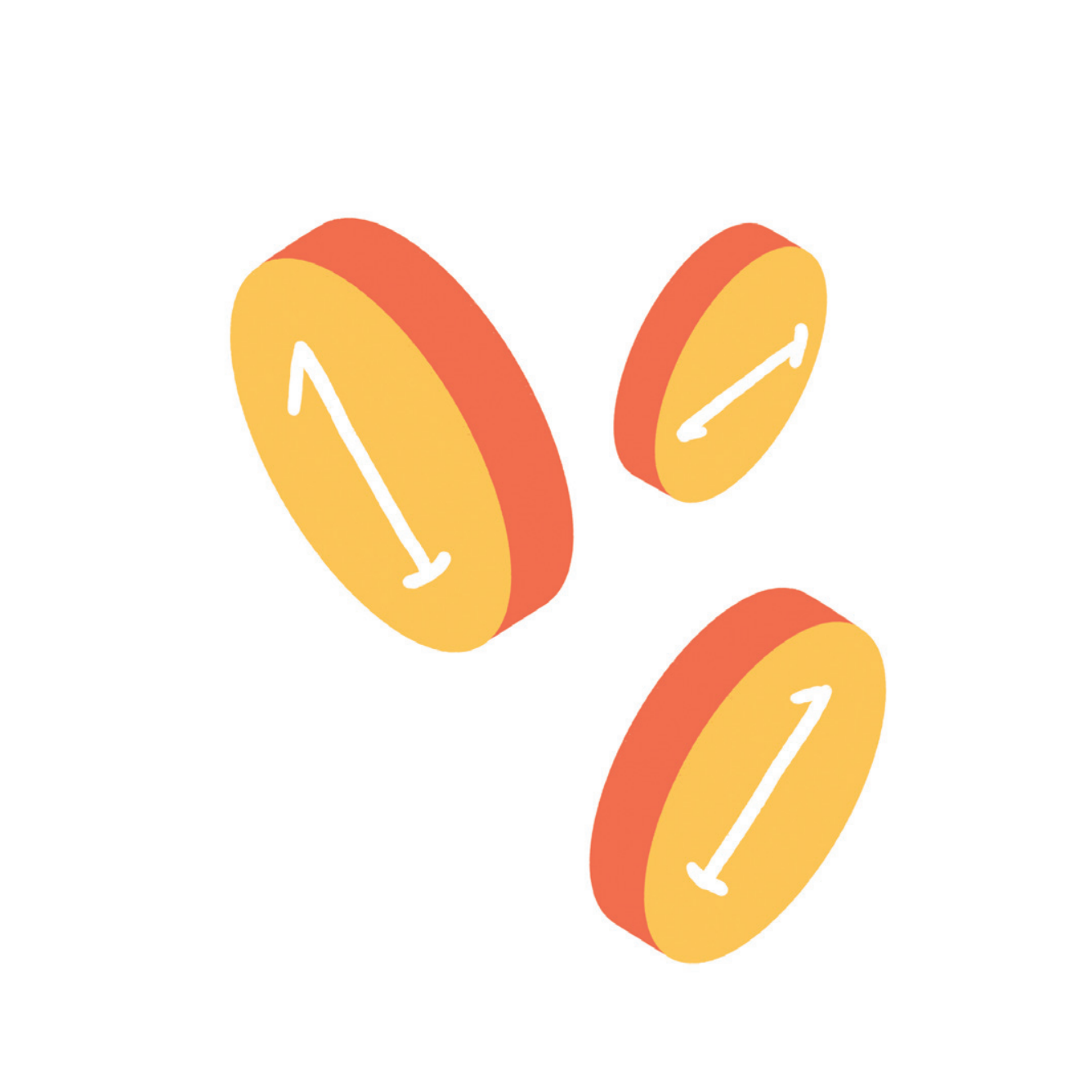




\section{CHAPTER 7}

\section{Feasibility of gamified cognitive}

training in Parkinson's disease: the randomized controlled Parkin'Play pilot 


\section{ABSTRACT}

Cognitive training (CT) shows modest positive effects on cognitive function in patients with Parkinson's disease (PD). Gamification may enhance adherence to traditional CT, but this has not been studied yet. Here, we investigated the feasibility of a gamified CT. We performed a randomized controlled trial including PD patients with mild cognitive impairment. Participants were randomly allocated to a 12-week home-based gamified CT intervention or waiting-list control group. Assessments were performed at baseline, and at weeks 12 and 24. Forty-one patients were included (21 intervention; 20 waiting-list controls). Sixty-three percent of the intervention group trained $>50 \%$ of the recommended sessions, while $81 \%$ voluntarily continued training after 12 weeks. After 24 weeks, $87.5 \%$ graded the game to be satisfactory. Global cognition scores improved after 24 weeks. Home-based gamified CT shows acceptable feasibility in patients with PD, and we observed preliminary indications for efficacy. Larger trials are needed to establish this efficacy. 


\section{INTRODUCTION}

Cognitive impairment is an important concern for patients with Parkinson's disease (PD) and decreases the quality of life [1]. Moreover, cognitive impairment may progress to dementia in advanced disease stages, causing considerable caregiver burden and ultimately resulting in institutionalization [2]. Treatments are therefore needed, also because early interventions might slow down progression to dementia [3]. Pharmacotherapy is not effective for mild cognitive impairment $(\mathrm{MCl})$ and has only limited effects on PD dementia. Cholinesterase inhibitors can delay cognitive decline up to at least six months, but increase the risk of adverse drug reactions [4]. Hence, nonpharmacological treatment for cognitive impairment might prove particularly meaningful. Recent work points to the possible beneficial effects of cognitive training (CT). CT appears to be safe and cost-effective in PD, with modest positive effects on executive functions and working memory [5]. Gamification may further enhance traditional $\mathrm{CT}$ in terms of attractiveness and adherence [6], thereby making patients more inclined to continue playing, increasing possible treatment effectiveness, and maintaining benefits over time. So far, the merits of gamified CT have never been investigated in PD patients. Our long-term aim is to perform a large randomized controlled trial to evaluate the impact of gamified CT on cognition in PD [7]. The focus of the present proof-of-principle study is to test the feasibility and adherence to gamified CT.

\section{PATIENTS AND METHODS}

A complete description of the methods of this randomized controlled trial has been published previously [7]. PD patients were recruited at three centers in the Netherlands. Inclusion criteria were PD (diagnosed by a neurologist), Hoehn \& Yahr stage $\leq 3$, age between 40 and 75 years, mild cognitive impairment according to MDS (MCI Level 1 criteria), and stable dopaminergic medication during the last 3 months. Patients with dementia were excluded. Eligible patients were randomized to either the gamified CT or no intervention (waiting-list control). The intervention group was asked to perform the online CT game (named AquaSnap ${ }^{\mathrm{TM}}$ ) at home using an internet-browser for a recommended 3 weekly sessions of 30 minutes each, for at least 12 weeks (primary phase). Participants scheduled their own agenda and each session duration was not fixed. Both groups could voluntarily play the gamified CT from weeks 12 to 24 (secondary phase). The waiting-list control group did not train in the primary phase, but was allowed to train in the secondary phase. AquaSnap ${ }^{\mathrm{TM}}$ is an adaptive CT game exercising five cognitive domains: attention, working memory, episodic memory, psychomotor speed and executive function. The player is an underwater photographer, exploring the ocean and completing cognitive tasks by taking pictures of fish. Pictures are worth currency and are used to progress into more difficult game levels. To promote adherence, various game elements are incorporated, such as goals, challenges/missions, reward systems, personalization and 3D environments. To assess the feasibility of standalone $\mathrm{CT}$, patients trained unsupervised at home and only reactive support was provided when requested by the patient. At baseline and after 12 and 24 weeks, a standard neuropsychological assessment battery including 
self-report questionnaires was administered in the ON medication state (Table 1), as well as an online cognitive assessment (MyCQ $\left.{ }^{\mathrm{TM}}\right)$. This 30-minute $\mathrm{MyCQ}^{\mathrm{TM}}$ assessment was included to automatically adapt the game difficulty level to the performance of the patient, thereby personalizing the intervention [8]. After completing a 12-week training period, participants completed an intervention acceptability questionnaire, in which they were asked to grade the overall intervention. The waiting-list control group completed this questionnaire 24 weeks after the randomization visit whereas the intervention group received the questionnaire after 12 weeks and 24 weeks. Assessors were not blinded to treatment allocation. Missing data were imputed prior to statistical analysis. To be able to replicate outcome measures and use a-priory defined criteria, four feasibility outcome measures (accessibility, training compliance, technical smoothness, and training motivation) were derived from previous studies on computerized CT $[9,10]$. The effect of gamified CT on global cognition at 12 and 24 weeks was analyzed using 2-tailed $t$ tests for independent samples.

\section{RESULTS}

Forty-one patients were included (21 intervention; 20 waiting-list controls). Baseline characteristics were similar between both groups, including cognitive status (Table 1). Three patients dropped out of the intervention group due to depressive symptoms $(n=1)$ or technical issues related to logging in and getting the game and assessment to work properly in their browsers ( $n=2$ ) (Table 1). One patient dropped out of the waiting-list control group for similar technical difficulties and two because of planned surgery.

During the primary phase from baseline to week 12 , the mean number of sessions per week within the intervention group was $2.9 \pm 3.4$ with a mean duration of $89 \pm 88 \mathrm{~min} /$ week (Table 1). In total, $35.5 \pm 30.4$ (range 17-120) sessions of the recommended 36 sessions (98.3\%) were performed during the primary phase. Six participants (37.5\%) performed more than 36 sessions, which was allowed, thereby increasing the average completion rate. When we cut off all number of sessions above 36 for these four participants, the intervention group performed on average $24.6 \pm 11.3$ (range 7-36) sessions (68.3\%). From between week 5 and 8 , the intervention group on average trained the required number of sessions, and this number decreased between week 17 and 20 . In terms of duration, eleven participants (68.8\%) trained more than $50 \%$ of the recommended training minutes in the primary phase, of which seven participants trained more than $100 \%$ (Appendix 1). Two intervention participants did not start training, because technical issues disallowed them to train; both subjects were therefore left out of the study. In the secondary and voluntary phase from week 12 to 24 , the intervention group trained $2.4 \pm 2.6$ sessions/week and $91 \pm 114 \mathrm{~min} /$ week. Three participants stopped with performing additional sessions after the primary phase (18.8\%). 


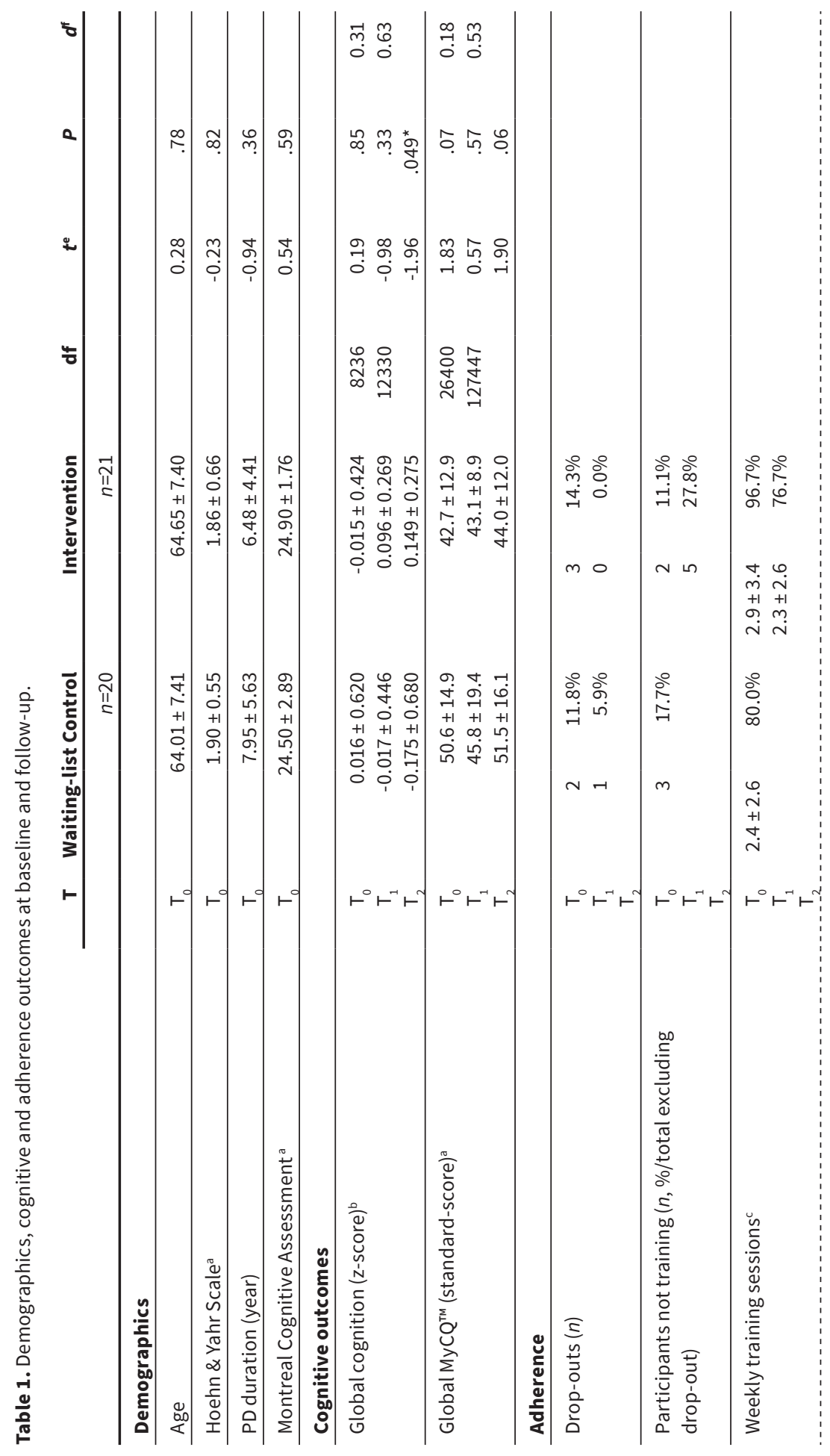




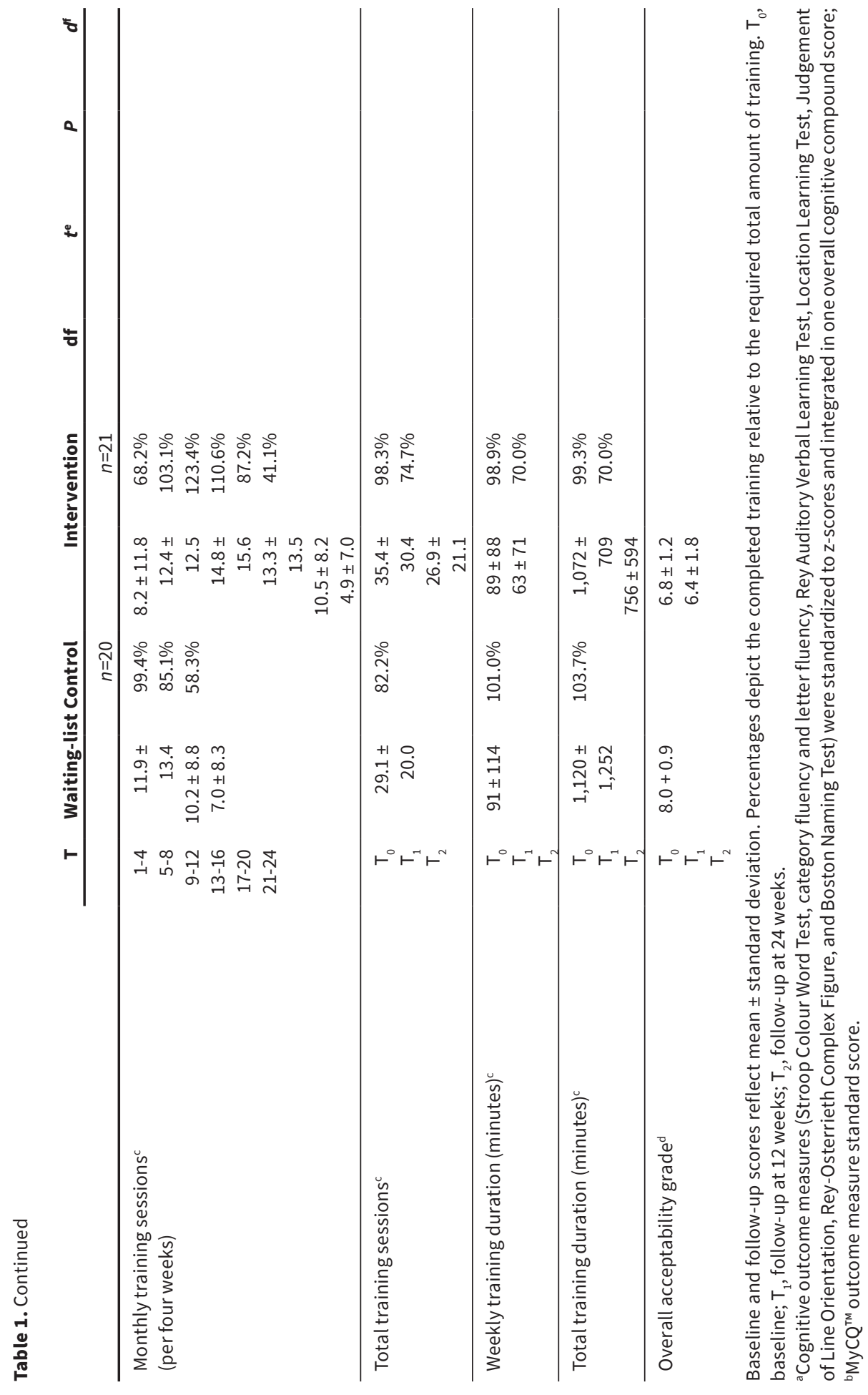


Table 1. Continued

${ }^{c}$ waiting-list control $n=14$ and intervention $n=16$, both intervention and waiting-list control patients were allowed to train voluntarily after $\mathrm{T}_{1}$;

'Overall acceptability grade on item: “What grade would you give the game intervention?". 10-point grading scale: $1=$ very poor, $6=$ satisfactory, $10=$ perfect;

e2-tailed $t$ for independent samples;

${ }^{\text {f}}$ Cohen's $d$. $T$ test results are depicted in the p values (<.05), which indicates group difference between intervention and waiting-list control group.

${ }^{*} P$ values $<0.05$ were considered significant.

Global cognition scores improved in the intervention group after 24 weeks of training compared to the waiting-list control group: respectively $0.149 \pm 0.275$ and $-0.175 \pm 0.680(t=-1.96, P=.049$, $d=0.63$; Table 1 ). However, no significant between group difference was observed at 12 weeks follow-up.

Feasibility outcome measures based on the intervention feasibility criteria by Verhelst et al. [10] are presented in Table 2. Most, but not all criteria were met: (1) all intervention group participants understood the goal of the game, (2) the average training completion for the intervention group after 12 weeks was $68.2 \%$ (SD = 31.3, range 19.4\%-100\%). Eight out of the 16 intervention participants carried out at least $86 \%$ of the sessions during the 12 -week training intervention, while three participants (18.8\%) completed less than $40 \%$ of the sessions. (3) In total, half of the participants encountered technical issues as a results of login difficulties and plugin support difficulties. Although these issues were resolved to prevent future interruptions, some were critical, since they resulted in drop-out $(n=2)$. (4) Most participants (87.5\%) gave at least a 'satisfactory' grade on the 10-point acceptability questionnaire, while only one (6.3\%) gave an 'unsatisfactory' grade. The mean acceptability questionnaire grade was $6.4 \pm 2.1$ (range 4-8). Twelve intervention participants (75\%) would recommend this game to others. In the secondary phase, the proportion of participants from the intervention group reporting to like playing the game increased from $47 \%$ to $64 \%$. In the waiting-list control group, which was allowed to play the game in the secondary phase only, the mean acceptability grade was $8.0 \pm$ 0.9 (range 6-10), indicating they appreciated the intervention more than the intervention group. 


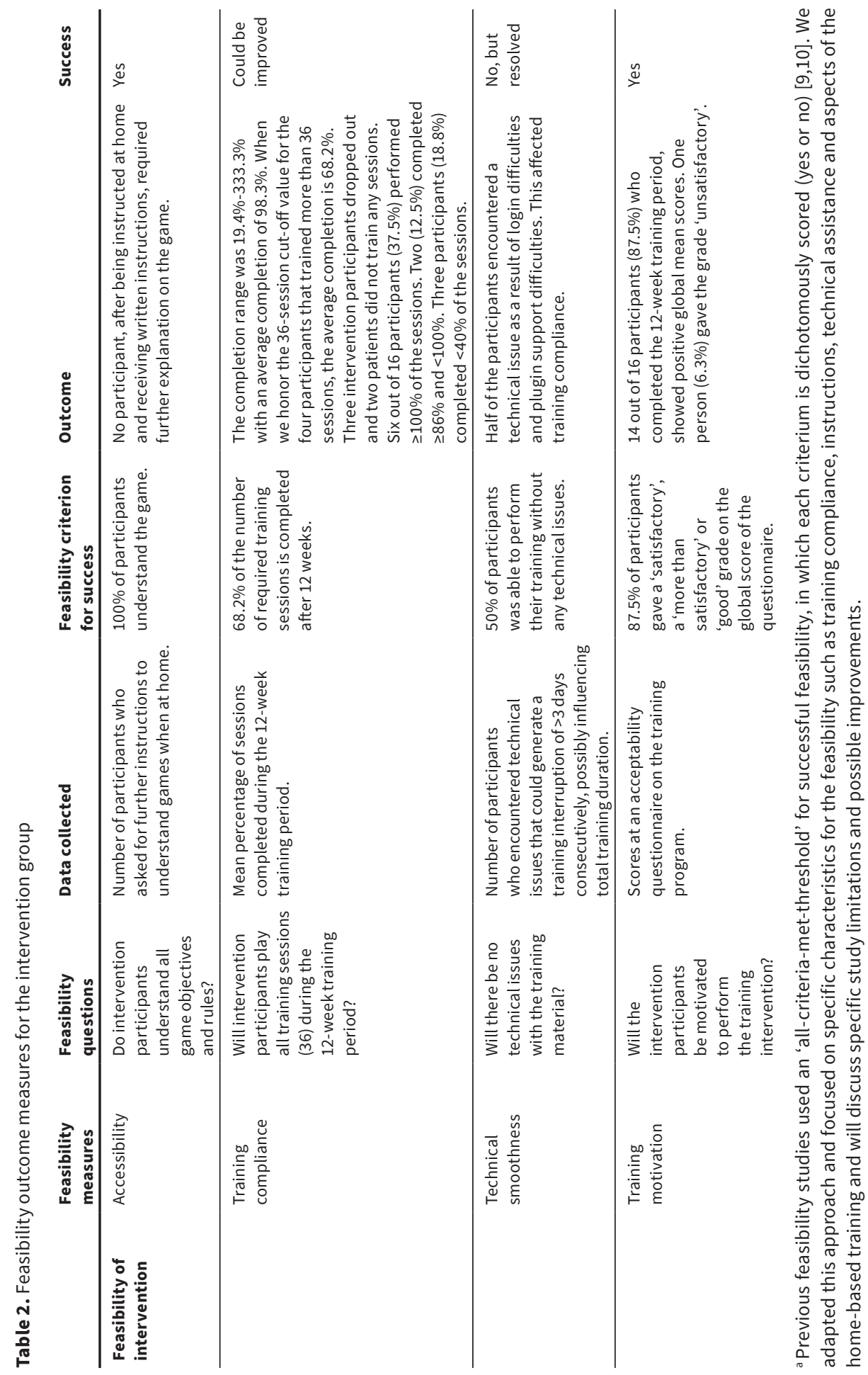




\section{DISCUSSION}

This study evaluated the feasibility of a home-based gamified cognitive training (AquaSnap ${ }^{T M}$ ) in a group of PD patients with $\mathrm{MCl}$. The results show an acceptable feasibility of this online cognitive training approach. As such, it holds promise for a future larger clinical trial. In order to reflect on the feasibility of the study, different aspects of both the cognitive training and the study design should be addressed.

When we reflect on the feasibility or our cognitive training, we found that the training compliance in general was high for both the intervention and waiting-list control group. However, we observed a considerable variation in the number of participants completing the recommended number of trainings.

In our study, sixty-three percent of the required training sessions was completed after 12 weeks and $69 \%$ of the participants played more than half of the prescribed training duration. The observed variation might be explained by the relatively non-committal nature of the training instructions. Although we recommended the participants to train 30 mins per session for 3 weekly sessions, this instruction was not strict and the training sessions have not been closely checked by the research staff. Hence, participants may have felt free to play according to their own needs, resulting in considerable variation. In comparison, in a 5-week computerized cognitive intervention pilot study in nine persons with Huntington's disease, $77 \%$ started the training and of those $100 \%$ completed all training sessions [11]. In another study of 59 persons with multiple sclerosis, $71 \%$ started the training and of those, $81 \%$ played more than half of the prescribed training sessions [12]. The results of the current study are in line with these observations, although the percentage of $\mathrm{PD}-\mathrm{MCl}$ patients playing more than half of the prescribed training was a bit lower in our study. This could be due to differences in patient profiles and age. We have no indications that the lower training adherence was due to unclear game objectives, since all participants responded to understand the game objectives and rules and none of our participants required additional game instructions. Study participants received both an extensive in-person training of the game (at home) and paper support manuals. This approach can be relevant for clinical practice, since high treatment complexity and limited treatment knowledge are barriers for therapy compliance [13].

Similar to some other studies, many participants experienced technical issues [14] and these likely hindered adherence rates and therapy compliance. Making the gamified cognitibe training available as an app for smartphones and tablets will presumably decrease technical problems and increase usability. For our cognitive training, this has meanwhile been adopted in a new version of the product. This is especially relevant since many $\mathrm{MCl}$ patients have compromised problem solving skills [15] and therefore are less likely to successfully solve technical issues. In addition, the investigated (now obsolete) version of the game was designed to be engaging for up to 18 training hours, after which no additional content was added to the gameplay. Consequently, some participants indicated that the game, at some point, had 
become monotonous. This is reflected in our results: between week 17 and 20, the number of sessions started to decrease. Since prolonged periods of training or repeated booster sessions are likely beneficial for long-term cognitive effects [16], future gamified interventions should include a sufficient amount of gameplay variance (levels, challenges, missions, positive feedback elements, social challenges, unlockable content, and continuous updates) to achieve optimal engagement. Nevertheless, almost all participants gave a satisfactory overall grade for the game and $75 \%$ would recommend this game to others. The more positive feedback of the waiting-list control group could be related to different expectations generated after the initial waiting period, the non-binding nature of the secondary phase, or the improved problem-solving skills of the support staff. Altogether, this indicates that this type of training can be considered relevant for this target population, however, it may only be suitable for a certain proportion of patients with PD-MCI. More research is needed to investigate for which portion of the PD-MCI population online cognitive training training is most suitable.

In other populations (such as non-PD dementia or healthy older adults), gamification and personalization of therapies are felt to be motivating and attractive $[17,18]$, but firm empirical evidence is still lacking [19]. In order to increase adherence, the gamified cognitive training used in this study incorporated an online cognition assessment (MyCQ ${ }^{\mathrm{TM}}$ ) that was used to adapt the training to the patients' cognitive abilities. As such, the cognitive training focused on personalized and engaging gameplay over longer periods of time. One of the most frequent reasons reported to decline participation in an intervention study is the need to travel to an institute for the assessments [9]. MyCQ ${ }^{\mathrm{TM}}$, when validated, can be particularly helpful in this regard, since cognitive assessments can be performed in the home-situation. Moreover, our patients could also train at home at any time. However, remote supervision or technical assistance for patients training at home could be of specific relevance for this group of patients. Whereas many previous studies provided remote supervision $[9,20]$, we intentionally only provided reactive technical support to test the stand-alone feasibility and adherence. Participants could schedule their own training-agenda, independently from research staff, and end sessions at the duration of their likings. On the other hand, the reactive technical assistance instead of pro-active remote supervision may have had a negative impact on adherence, as observed in the considerable variation in number of training sessions and training duration. The advantages of remote supervision are better monitoring of progression, the possibility to give personalized feedback, and motivational interviewing to boost adherence $[9,11,15]$. Additionally, participants can be given a more structured and scheduled therapy with regard to timing of session numbers and durations. Thus, a blended approach combining personalized and engaging gamified cognitive training in the home-situation with low-threshold remote supervision seems to have great future potential.

Our primary goal was to test the feasibility of a gamified cognitive training. Secondarily, we also explored potential effects and our analyses showed a significant improvement of global cognition scores after 24 weeks. This effect was not found after 12 weeks. The significant improvement increased in subgroup analysis (2-tailed $t$-tests for independent samples), 
in which the intervention group was compared to a passive control group (e.g. only the participants in the waiting-list control group who were inactive in the secondary phase were included in the control group). This may suggest that gamified cognitive training interventions should be executed a minimum (cumulative) amount of time to possibly affect cognition, and that continuous training may be essential for prolonged cognitive benefits over time. Previous traditional or computerized cognitive training programs also found small to modest effects of cognitive training interventions on cognition in PD, mainly on measures of processing speed, working memory, and executive functions [5]. However, none of those used a home-based gamified and personalized cognitive training, and most trials used heterogeneous cognitive outcomes and short follow-up periods, hampering a direct comparison with these studies. Although this proof-of-principle study included a relatively large sample of participant when compared to other cognitive training interventions, larger studies with robust cognitive outcomes that also focus on translational aspects into daily life are needed to determine the effectiveness of this intervention. Additionally, trials should include blinded assessors, an active control group receiving a mock intervention and longer follow-up periods.

In conclusion, this study shows that PD patients with $\mathrm{MCl}$ can perform an online, gamified and unsupervised cognitive training on a regular basis in their homes, with a potential effect on global cognition after 24 weeks of training. Intervention feasibility criteria are mostly met, but improvements are needed in order to increase usability. Even if online gamified cognitive training may not be suitable for all patients, many PD patients are interested in such interventions [6], increasing the need to further investigate the efficacy of gamified cognitive training in larger PD trials. 


\section{APPENDIX}

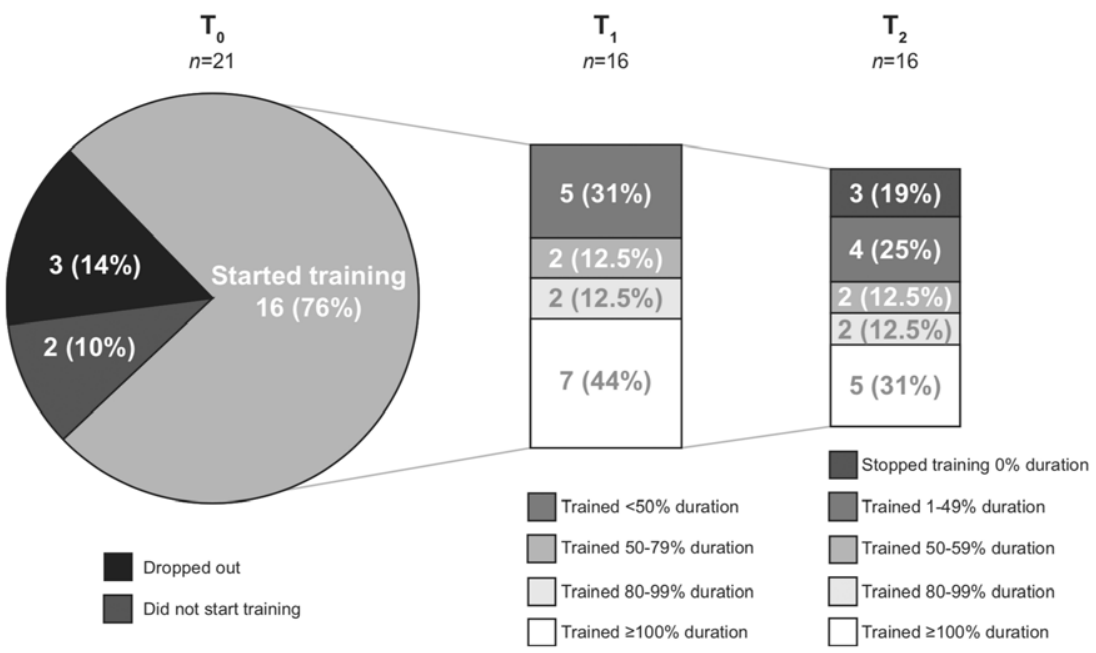

Appendix 1. Dropout and training adherence for the intervention group at baseline and follow-ups. $\mathrm{T}^{0}$, baseline; $\mathrm{T}^{1}$, follow-up at 12 weeks (primary phase); $\mathrm{T}^{2}$, follow-up at 24 weeks (voluntary secondary phase). Two intervention patients did not train, but completed all assessments.

\section{ACKNOWLEDGEMENTS}

We thank Mirella Davies-Waber, Vienna Kooijman, Amée Wolters, Joni de Kriek, Ashley van Woerkom, Tiny Sporken, and Jessica Hubbers for their contribution in this study.

\section{STATEMENT OF ETHICS}

All subjects gave their written informed consent and the study protocol was approved by the institute's committee on human research: METC azM Maastricht, number NL51188.068.14.

\section{DISCLOSURE STATEMENT}

All support and financial involvement of the authors will be added here after the first review round. The authors have no conflicts of interest to declare.

\section{FUNDING SOURCE}

The Parkin'Play study was funded by MyCognition, London, UK. The sponsors had no role in the design of the study and collection, analysis, and interpretation of data, and in writing the manuscript.

\section{AUTHOR CONTRIBUTIONS}

SW, AD BB, GT, and MK were involved in the conception, organization and execution of the research project. SW and SK were involved in the execution of the statistical analysis. RK and GT were involved in the organization of the research project. All authors were involved in the review of the analysis and the writing of the manuscript. 


\section{REFERENCES}

1. Schapira AHV, Chaudhuri KR, Jenner P. Non-motor features of Parkinson disease. Nature Reviews Neuroscience. Nature Publishing Group; 2017 Jun 8;18(7):435-50.

2. Schrag A, Hovris A, Morley D, Quinn N, Jahanshahi M. Caregiver-burden in parkinson's disease is closely associated with psychiatric symptoms, falls, and disability. Parkinsonism and Related Disorders. 2006 Jan;12(1):35-41.

3. Azuma T, Cruz RF, Bayles KA, Tomoeda CK, Montgomery EB. A longitudinal study of neuropsychological change in individuals with Parkinson's disease. International journal of geriatric psychiatry. Wiley-Blackwell; 2003 Nov;18(11):1043-9.

4. Pagano G, Rengo G, Pasqualetti G, Femminella GD, Monzani F, Ferrera N, Tagliati M. Cholinesterase inhibitors for Parkinson's disease: a systematic review and meta-analysis. Journal of Neurology, Neurosurgery \& Psychiatry. BMJ Publishing Group Ltd; 2015 Jul;86(7):767-73.

5. Leung IHK, Walton CC, Hallock H, Lewis SJG, Valenzuela M, Lampit A. Cognitive training in Parkinson disease: A systematic review and meta-analysis. Neurology. 2015 Oct 30;85(21):1843-51. PMCID: PMC4662707

6. Van de Weijer SCF, Kuijf ML, de Vries NM, Bloem BR, Duits AA. Do-It-Yourself Gamified Cognitive Training: Viewpoint. JMIR Serious Games. 2019 May 7;7(2):e12130.

7. Van de Weijer SCF, Duits AA, Bloem BR, Kessels RP, Jansen JFA, Köhler S, et al. The Parkin'Play study: protocol of a phase II randomized controlled trial to assess the effects of a health game on cognition in Parkinson's disease. BMC Neurol. BioMed Central; 2016 Nov 3;16(1):209. PMCID: PMC5093943

8. Domen AC, van de Weijer SCF, Jaspers MW, Denys D, Nieman DH. The validation of a new online cognitive assessment tool: The MyCognition Quotient. Int J Methods Psychiatr Res. John Wiley \& Sons, Ltd; 2019 Feb 13;6(10):e1775.

9. Corti C, Poggi G, Romaniello R, Strazzer S, Urgesi C, Borgatti R, Bardoni A. Feasibility of a homebased computerized cognitive training for pediatric patients with congenital or acquired brain damage: An explorative study. van Wouwe JP, editor. PLoS ONE. Public Library of Science; 2018;13(6):e0199001. PMCID: PMC6010294

10. Verhelst H, Vander Linden C, Vingerhoets G, Caeyenberghs K. How to Train an Injured Brain? A Pilot Feasibility Study of Home-Based Computerized Cognitive Training. Games Health J. Mary Ann Liebert, Inc. 140 Huguenot Street, 3rd Floor New Rochelle, NY 10801 USA; 2017 Feb;6(1):28-38.

11. Sadeghi M, Barlow-Krelina E, Gibbons C, Shaikh KT, Fung WLA, Meschino WS, et al. Feasibility of computerized working memory training in individuals with Huntington disease. Fasano A, editor. PLoS ONE. Public Library of Science; 2017;12(4):e0176429.

12. Shatil E, Metzer A, Horvitz O, Miller A. Home-based personalized cognitive training in MS patients: a study of adherence and cognitive performance. NeuroRehabilitation. 2010;26(2):143-53.

13. Jin J, Sklar GE, Min Sen Oh V, Chuen Li S. Factors affecting therapeutic compliance: A review from the patient's perspective. Ther Clin Risk Manag. Dove Press; 2008 Feb;4(1):269-86. 
14. Manera V, Ben-Sadoun G, Aalbers T, Agopyan H, Askenazy F, Benoit M, et al. Recommendations for the Use of Serious Games in Neurodegenerative Disorders: 2016 Delphi Panel. Front Psychol. Frontiers; 2017;8:1243.

15. Anderson RJ, Simpson AC, Channon S, Samuel M, Brown RG. Social problem solving, social cognition, and mild cognitive impairment in Parkinson's disease. Behav Neurosci. 2013 Apr;127(2):184-92.

16. Rebok GW, Ball K, Guey LT, Jones RN, Kim HY, King JW, et al. Ten-year effects of the advanced cognitive training for independent and vital elderly cognitive training trial on cognition and everyday functioning in older adults. J Am Geriatr Soc. 2nd ed. John Wiley \& Sons, Ltd (10.1111); 2014 Jan;62(1):16-24. PMCID: PMC4055506

17. Kappen D, Mirza-Babaei P, Nacke L. Gamification of Older Adults' Physical Activity: An Eight-Week Study. HICSS. Hawaii International Conference on System Sciences; 2018. p. 1-11.

18. Forman EM, Goldstein SP, Flack D, Evans BC, Manasse SM, Dochat C. Promising technological innovations in cognitive training to treat eating-related behavior. Appetite. 2018 May 1;124:68-77. PMCID: PMC5641227

19. Lumsden J, Edwards EA, Lawrence NS, Coyle D, Munafò MR. Gamification of Cognitive Assessment and Cognitive Training: A Systematic Review of Applications and Efficacy. JMIR Serious Games. JMIR Publications Inc., Toronto, Canada; 2016 Jul 15;4(2):e11. PMCID: PMC4967181

20. Van der Kolk NM, de Vries NM, Penko AL, van der Vlugt M, Mulder AA, Post B, et al. A remotely supervised home-based aerobic exercise programme is feasible for patients with Parkinson's disease: results of a small randomised feasibility trial. Journal of Neurology, Neurosurgery \& Psychiatry. BMJ Publishing Group Ltd; 2017 Jun 12; jnnp-2017-315728. 


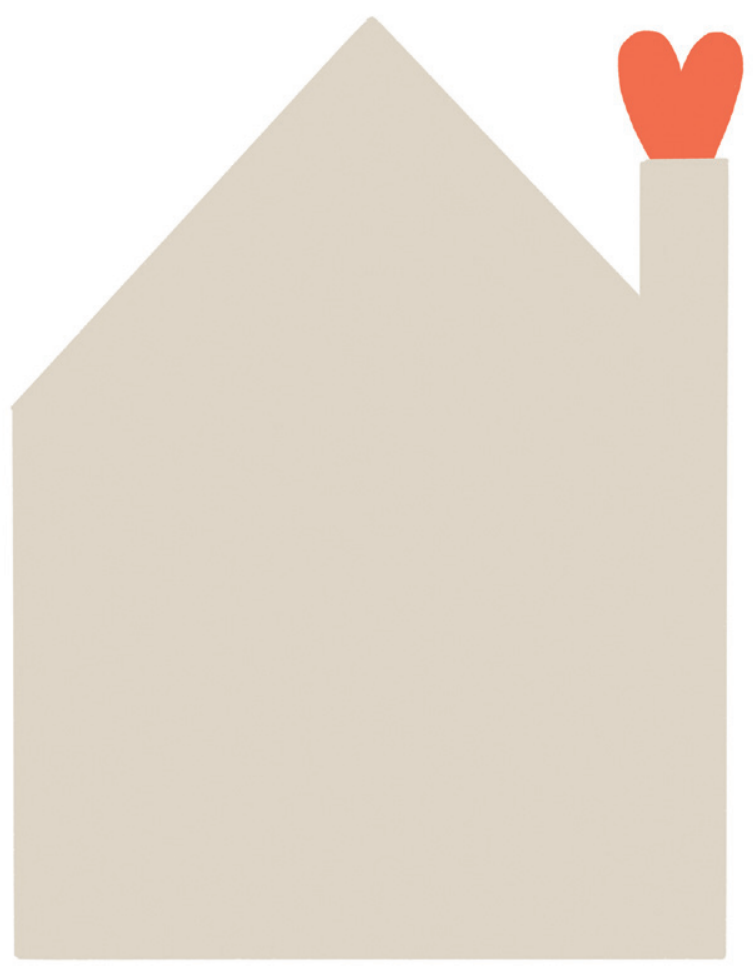




\section{CHAPTER 8}

\section{Summary, General Discussion, and Potential Impact}




\section{SUMMARY}

\section{SUMMARY IN ENGLISH}

The main aim of this thesis was to investigate the prerequisites for gamification in homebased cognitive training and analyze the adherence to such interventions in persons with Parkinson's disease (PD). These answers could help to establish new home-based cognitive treatment programs. This thesis has evolved around the Parkin'Play study, which aimed to test the adherence to and efficacy of a gamified and home-based cognitive training in a PD-MCI population.

\section{COGNITIVE DECLINE AND ITS UNDERLYING PATHOPHYSIOLOGY}

In Chapter 2, we outlined the problem of cognitive decline in PD and investigated the current knowledge of the biological basis for cognitive training in PD. Cognitive impairment is a common non-motor symptom in PD that results in impairments in attention, memory, processing speed, executive functioning, and visuospatial orientation skills. Up to $80 \%$ of the population develops dementia during the course of the disease. The underlying pathophysiology presently remains unclear. Identifying a valid biomarker for cognitive decline could provide new steppingstones for cognitive training. The clinical manifestations of PD are mainly related to reduced dopamine production in the substantia nigra, resulting in difficulties controlling movements. Importantly, dopamine does not solely play a role in motor functions. Even in early stages of PD, alterations can occur in executive functions and visuospatial abilities, including planning and working memory, suggesting a role for dopamine in controlling cognitive functions. This assumption is supported by the observation that that levodopa treatment can improve cognitive functions that are controlled by dopamine-depleted brain regions but can decrease cognitive functions that depend on non-depleted regions (i.e. an inverted-U relation) [1]. Nonetheless, the pathophysiology underlying cognitive decline in PD remains largely unclear and no valid biomarkers for cognitive decline in PD have been identified. Further research in this field has focused on the formation of protein aggregates, on neurotransmitter system dysfunction other than dopamine and on genetic risk factors. More recently, the "dual syndrome hypothesis" has been introduced that suggests (1) a role for dopaminergic dysfunction in planning, working memory and executive functions, and (2) a role for cholinergic dysfunction in visuo-spatial function and semantic fluency. The latter syndrome may lead to a more rapid cognitive decline to dementia [2].

Several studies have used functional magnetic resonance imaging ( $\mathrm{FMRI}$ ) to investigate brain connectivity changes in PD. A number of alterations in functional network connectivity have been described in PD patients with cognitive impairment which are probably the result of the heterogenous pathophysiology underlying this cognitive decline, including dopaminergic and cholinergic deficits. Accordingly, the reported resting-state connectivity patterns vary greatly among studies. 
We aimed to evaluate the localization and magnitude of functional connectivity patterns in resting-state brain networks in PD patients with cognitive impairment by pooling data from available studies, in order to obtain a better understanding of the functional connectivity networks that play a role in PD with cognitive decline, and to investigate the usability of fMRI results as a biomarker for PD with cognitive decline. A literature search in PubMed, Medline, Embase, PsycINFO and the Cochrane Library was performed to identify functional MRI studies in PD patients with cognitive impairment. Next, the reference lists of the included articles were scanned for additional qualified manuscripts. We conducted the search on the 30th of April 2018, resulting in a total of 1,122 articles. Resting-state fMRI studies were included that compared a group of cognitively impaired PD patients with either PD patients without cognitive decline or healthy controls. Only studies were included that applied a whole-brain analysis, independent component analysis or seed-based analysis, with a correlation of the seeds to voxels encompassing the entire brain. After exclusion, screening, and reading of the manuscript, a total of seventeen studies were used in the analysis. Combined, these studies included 932 participants.

We derived our own criteria for assessing the quality of $\mathrm{FMRI}$ studies from the guidelines for reporting fMRI studies as described by Poldrack et al. [3] and assessed the quality of all papers. Using the anisotropic effect-size version of the signed differential mapping method, a voxelbased meta-analysis combined with quality statistics was performed, which SDM is specifically developed to combine neuroimaging studies with studies reporting solely peak coordinates in coordinate systems (e.g. Talairach, MNI).

In the default mode network, right fronto-parietal network and auditory network, we found reduced connectivity when comparing PD patients with cognitive impairment with healthy controls. Furthermore, when PD patients with cognitive impairment were compared with healthy controls, we also saw signs of spatial correlation with the sensorimotor network, potentially related to the motor symptoms of patients with PD. For the comparison of PD who are cognitively unimpaired with PD patients with cognitive impairment, we found reduced connectivity especially in the default mode network. These networks are thought to be relevant for cognition. Taken together, our pooled findings provide a distinct step in the differentiation of network disruptions associated with cognitive impairment in PD. Ultimately, functional connectivity alterations may contribute to cognitive decline in Parkinson patients and may be a promising future biomarker, although much more work remains needed to translate these accrued group data to something that is usable at the level of individual patients.

Take-home message:

1. A biomarker for cognitive decline could provide new targets for cognitive training.

2. In cognitively impaired PD patients, reduced connectivity was found in the default mode network.

3. Larger studies are needed to explore the potential role of fMRI as a biomarker for cognitive decline. 


\section{CURRENT KNOWLEDGE ON COGNITIVE TRAINING}

In Chapter 3, we elaborated on the current knowledge as well as future directions for cognitive training in PD. Increasingly valuable treatment options to overcome or reduce problems in patients with PD are non-pharmacological interventions. More and more supportive evidence is emerging for such interventions, among which are occupational therapy, physiotherapy, exercise, diet, non-invasive brain stimulation, etcetera [4]. PD patients are at risk of cognitive decline in both earlier and later stages of the disease. Common cognitive deficits in PD include impairment in cognitive flexibility, reasoning, attention, planning, and verbal processes [5]. A growing body of evidence is emerging for the positive effects of cognitive training on working memory, processing speed, and executive function in PD [6], and hence, cognitive training is increasingly being acknowledged as a treatment option for cognitive decline in patients with PD. With modest positive effects on working memory as well as executive functions, cognitive training appears to be safe and cost-effective in PD [7]. However, a drawback of these traditional cognitive training programs is the need for repetitive execution of cognitive tasks which may become monotonous over time, creating a risk of nonadherence. New technological innovations may further enhance traditional cognitive training in terms of attractiveness and adherence, thereby making patients more inclined to continue playing, to increase possible treatment effectiveness, and to maintain benefits over time. An example of a relatively new intervention is computer-based cognitive training is, which includes innovative elements such as adaptive and personalized training, with the added benefit of providing preventative care to the homes of those who need it. In Chapter 7, we presented the feasibility results of the interim analysis of the Parkin'Play study. The results additionally indicated that gamified home-based cognitive training might have beneficial effects on overall cognition in PD. In a recent review that included eight studies that used computer-based cognitive training in PD, positive effects were found in memory, processing speed, executive function and attention [5]. In addition to making cognitive training computer-based, artificial intelligence and virtual reality could boost the efficacy of such innovative therapies. These recent technological innovations make it possible to create flexible computer-based and home-based trainings, adding benefits over inperson cognitive training in terms of scalability, cost-effectiveness, flexibility, and continuous tailoring in training content.

This is not only relevant for early-stage PD, but also for patients with late-stage PD. By including more late-stage PD patients in future studies on non-pharmacological treatments in PD in general (and on computer-based cognitive training in particular), we could increase the treatment options for the total PD population. In Chapter 3, we presented several (noncomprehensive) recommendations for future gamified cognitive training design for late stage PD. For example, the user interface needs to be comprehensible and simple, and keyboard and mouse use needs to be tailored to the limitations in the musculoskeletal system control. Also, adaptation mechanism could be included and, in the future, further automated by Artificial Intelligence. A strong focus needs to be put on encouraging, positive feedback mechanisms that respond immediately to the player's inputs. Virtual reality could be used by creating realistic 3D environments within the intervention, which could possibly promote generalization 
or transfer outside of the training situation [8]. Many advancements are expected soon in terms of technological developments in cognitive training. Improved scientific studies could result in an increased treatment repertoire for PD in both the early and late stages of the disease.

Take-home message:

1. Cognitive training seems to be effective in persons with $P D$, but also has some drawbacks, such as the requirement to complete repetitive and mundane tasks for longer periods of time.

2. Many technological advancements, such as personalization of training, artificial intelligence, and virtual reality, are expected to be implemented in computer-based cognitive training in the near future.

3. Our recommendations, which focus on optimizing computer-based cognitive training, could be used in future designs of gamified cognitive trainings for both early and late stage PD.

\section{GAUGING INTEREST IN GAMIFIED THERAPIES}

Chapter 4 reports on the attractiveness of and motivation for gamified cognitive training in the PD population. Most of the cognitive trainings are performed under supervision. Additionally, most are merely (traditional) paper-pencil cognitive training programs presented as a computerized version, lacking rewarding gamification stimulants that could help to boost adherence. In Chapter 4, we described three patients with PD with different ages, from various backgrounds, and in different disease stages. Using computer videogames, real-life routines, or card games, these patients were able to self-train their cognitive abilities. Hereby, we intented to raise awareness for the potential of gamified cognitive training in PD patients and the importance of both personalization and cocreation in the development of games for health. To indicate generalization into a larger PD population, the recruitment results from a recent cognitive game trial are added.

Many similarities are evident in these three cases. First, all three patients were aware of their decline in cognitive abilities and were experimenting with solutions to potentially counteract their looming cognitive decline. Second, in order to improve their cognitive deficits, all three patients included a form of play in their daily routines. Finally, our results indicated that all patients were able to continue their training for years. They were intrinsically motivated to continue, possibly because they felt that the therapy had a positive effect on their functional performance. We also reported the results of a recruitment procedure in a recent gamified cognitive training study: a larger PD population appears to be interested in home-based cognitive training. Gamification may improve traditional therapies in terms of personalization and adherence. Although no gamified cognitive training study results in PD have been published to date, our findings in Chapter $\mathbf{4}$ advocate that PD is an attractive area to develop and test gamified cognitive trainings. This suggests an unmet need in this area and may justify the development of gamified cognitive training and its evaluation, for which our recommendations could be used. 
Take-home message:

1. Some patients are creating cognitive trainings themselves, and a larger group shows interest in gamified cognitive training trials as well.

2. Personalization seems to be an important factor in ascertaining the success of home-based interventions.

3. We recommend cocreation by various stakeholders in the development of games for health.

4. There is a need for the development and evaluation of more structured and gamified ways of training cognition in PD.

\section{ASSESSMENT OF COGNITION}

Chapter 5 evaluated the validation of the MyCognition Quotient (MyCQ): a brief online cognitive assessment, which is used in the Parkin'Play study to tailor the training program to the individual cognitive performance levels of a subject. Of course, cognitive impairment is not only relevant for PD patients. Cognitive impairment should be a treatment focus for psychiatric patients as well, while it influences their daily functioning. Reliably assessing cognitive status is important in both clinical practice as well as in a research setting, since it can assist in the diagnosis of the disease, the development of interventions (adaptation mechanisms), and the evaluation of treatment programs. Although assessment of cognitive status is crucial in cognitive remediation studies, current test batteries have several limitations. For example, most instruments are labor-intensive, expensive, and confined to a clinical setting. A further problem is that researchers can use a heterogenous collection of cognitive tests. This hinders comparability between studies. Several standardized computerized cognitive assessments are available already, but they are still dependent on relatively expensive soft- and hardware configurations. In Chapter 5, we presented a new, online tool, the MyCognition Quotient (MyCQ), which was developed to assess cognition in a fast and efficient way (within 30 minutes). It consists of 10 sub-tests that aim to assess five primary cognitive domains: episodic memory, working memory, psychomotor speed, attention, and executive functioning. The MyCQ results can not only be used to track cognitive status, but also to frequently personalize a cognitive training based on cognitive performance levels. We present the psychometric properties and aim to determine the validity of the MyCQ by comparing it with the Cambridge Neuropsychological Automated Test Battery (CANTAB), which is a computerized test kit that has been widely used in clinical practice as well as in scientific studies in a wide range of disorders and healthy controls, but takes up to three hours to complete.

We included eighty-seven psychiatric patients with major depressive disorder, obsessive compulsive disorder, or schizophrenia/schizoaffective disorder. Patients completed selected CANTAB sub-tests followed by the MyCQ on a laptop in the hospital. During the full assessment, an educated psychology student was present to supervise tasks and to make sure instructions were understuud. Principal component analysis comfirmed the domains of episodic memory, working memory, psychomotor speed, attention, and executive functioning. A high correlation was found for the MyCQ total score correlated and the CANTAB total score. The MyCQ domains 
of attention, psychomotor speed, and episodic memory showed moderate to high correlations with corresponding CANTAB domains. We found limited divergent validity for executive functioning and working memory.

Based on our study results, the MyCQ appears to be a valid, consistent and quick assessment of cognitive functioning within a mixed psychiatric population. Compared with traditional instruments, the MyCQ is able to measure overall cognition within a relatively short time and can be self-administered and accessed online. This creates possibilities for future home-based diagnostics and reduced costs. Similarities between psychiatric patients and PD patients exists in dopaminergic alterations, such a as dopaminergic denervation (PD), reduced dopamine signaling (depression and obsessive-compulsive disorder), and dopamine dysregulation (schizophrenia). These conditions all show reduced dopamine levels as well as decreased reward anticipation and processing, which might mean that faster (more rewarding) assessments could result in more reliable outcomes. More studies with different populations, for example, persons with PD (see Chapter 5) and breast cancer patients with depression, and different neuropsychological assessment batteries are in progress to further validate the MyCQ and investigate its usability within other populations.

Take-home message:

1. A brief, web-based cognitive assessment can be performed by a heterogenous group of psychiatric patients.

2. MyCQ seems to be a valid instrument to assess cognitive status in a psychiatric population, but it should be further validated against a wide array of neurocognitive tests and in other patient groups.

3. MyCQ is a promising instrument for a cost-effective and consistent assessment of cognitive status.

\section{INVESTIGATING THE EFFECTIVENESS OF GAMIFIED HOME-BASED COGNITIVE TRAINING}

In Chapter 6, we presented the design of the Parkin'Play study, which was designed to investigate the feasibility and the effects of a gamified home-based cognitive training intervention on cognition in PD.

The Parkin'Play Study aimed to recruit 222 PD patients with mild cognitive impairment in a multicentre, phase-II, open-randomized clinical trial. Eligible patients had PD, Hoehn \& Yahr stages I-III, were aged between 40 and 75 years, and had cognitive impairment but no dementia. Eligibility criteria were strict, because we wanted to obtain a homogeneous group in order to increase the chances of finding a possible effect. It was designed to include a total of 222 patients. The intervention group $(n=111)$ was scheduled to be trained using a web-based gamified cognitive training targeting multiple cognitive domains. The control group $(n=111)$ was planned to be placed on a waiting list. In order to increase compliance, the health game adapted to the subjects' performance, was enjoyable, and home-based. From each group, 
20 patients were scheduled to undergo fMRI to test for potential functional brain changes underlying treatment.

The primary endpoint was cognitive function after 12 weeks of training, as assessed by a standard neuropsychological assessment battery and an online cognitive assessment. The neuropsychological assessment battery assesses the domains of visual perception, visuoconstruction, executive function, memory, and language. We calculated a compound score for overall cognitive function as the mean score of all test Z-scores based on the distribution of scores for both groups taken together. Secondary endpoints were assessed at follow-up visits up to 24 weeks and included various motor and non-motor symptoms, biological endpoints (fMRI), and adherence.

\section{ADHERENCE TO GAMIFIED COGNITIVE TRAINING IN PD}

Gamification could potentially enhance traditional cognitive training with regard to attractiveness and adherence [9]. This may results in more patients who are willing to continue playing, increase possible treatment effectiveness, and maintain benefits over time. So far, the merits of gamified cognitive training have never been investigated in PD patients. We interim analyzed the feasibility of a gamified cognitive training in $\mathrm{PD}$, of which the results are presented in Chapter 7.

We performed a randomized controlled trial in which PD patients with mild cognitive impairment were randomly allocated to a home-based gamified cognitive training intervention with a duration of 12 weeks. Patients were asked to exercise the gamified cognitive training at home for 3 weekly sessions of 30 mins per session, while others were assigned to the waitlist-control group. Cognitive assessments were performed in the hospital at baseline, and at weeks 12 and 24. Both groups could voluntarily play the gamified cognitive training from weeks 12 to 24 .

Forty-one patients were included in the interim analysis ( 21 intervention; 20 controls). Baseline characteristics were comparable between both groups, including cognitive status. In the intervention group, $69 \%$ trained more than $50 \%$ of the recommended training minutes, while $81 \%$ voluntarily continued training after 12 weeks. After 24 weeks, $87,5 \%$ graded the game to be satisfactory.

The results show that gamified cognitive training appears feasible in PD patients with $\mathrm{MCl}$, with an acceptable adherence: PD patients can perform the prescribed number of weekly sessions and continue training for many weeks. The relatively high percentage of patients in the intervention group (81\%) who voluntarily continued training in the secondary phase indicates that the current intervention is sufficiently attractive, although drop-out and adherence rates could still be improved further. Reporting of adherence rates in computerized cognitive training is essential but remains rare. In previous home-based cognitive interventions in multiple sclerosis [10] we found comparable results to our interim analysis results. 
Encouragingly, we found preliminary indications for improved global cognitive function for the intervention group after 24 weeks of training. Nevertheless, larger trials are needed to establish efficacy of gamified cognitive training.

Take-home message:

1. It is feasible for PD patients to perform a gamified cognitive training for many weeks in the home situation.

2. Improvements may need to be made in future platforms to improve adherence rates, such as adding remote support / coach guidance and accountability elements.

3. It is too early to establish the efficacy of gamified cognitive training in PD.

\section{WHAT WE LEARNED}

In this thesis, we aimed to address the feasibility and efficacy of gamified home-based cognitive training in PD. This study consisted of three phases:

Phase A The development of a gamified and home-based cognitive training in collaboration with patients and MyCognition Ltd., aimed at creating a science-based and attractive training program that is both attractive to play and effective.

We learned We collaborated with MyCognition Ltd. and a game developer to develop the gamified cognitive training Aquasnap. The concept version was tested by PD patients in Maastricht and went through a couple of iterations. We additionally found it important to investigate the theoretical background of cognitive decline in PD and to research the interest of a PD population in such interventions. We found reduced connectivity in the default mode network in cognitively impaired PD patients. Furthermore, some PD patients are creating cognitive trainings themselves, and a larger group shows interest in gamified cognitive training trials.

Phase B-1 The conduction of a pilot study on the feasibility of such a gamified cognitive training in $\mathrm{PD}-\mathrm{MCl}$, in which the adherence to a 24-week long training program was evaluated.

We learned In our trial, PD patients were able to exercise a gamified training for many weeks in the home situation. We conclude that gamified cognitive training holds promise as a feasible approach in persons with PD. There were some factors that possibly negatively influenced our adherence numbers. Hence, future gamified interventions should consider adding more training programs, remote support / coach guidance and accountability elements. 
Phase B-2 The conduction of a pilot study on the preliminary effects of such a gamified cognitive training. An active intervention group was to be compared to a waiting list control group after 12 weeks of home-based gamified cognitive training (aimed at exercising the cognitive domains of working memory, episodic memory, attention, psychomotor speed and executive function).

We learned We carefully note that our gamified cognitive training has a small positive effect on global cognition, because the effect is only slightly significant for this sample size $(p=.49)$. This encourages us to strive for a larger trial, in which efficacy may be established.

Phase C The conduction of a larger study on the efficacy of an improved gamified home-based cognitive training in PD-MCl, in which all lessons learned from the previous phases were incorporated into an improved training program and study design.

We learned Because we did not reach this phase yet, we cannot establish the efficacy of gamified cognitive training in PD yet. In a future trial, we advise researchers to incorporate, among others, training programs with more variation in gameplay, a blinded assessor, remote support, and an active control group. 


\section{NEDERLANDSE SAMENVATTING}

Het voornaamste doel van dit proefschrift was het verkennen van de voorwaarden voor gamificatie in cognitieve training vanuit thuis, en om de therapietrouw te analyseren voor zulke interventies in een doelgroep van mensen met de ziekte van Parkinson. De antwoorden kunnen bijdragen in de ontwikkeling van nieuwe cognitieve behandelprogramma's die in de thuissituatie toegepast kunnen worden. Aan de basis van dit proefschrift ligt de Parkin'Play studie, dat deze doelen najoeg.

\section{DE ONDERLIGGENDE PATHOFYSIOLOGIE VAN COGNITIEVE ACHTERUITGANG}

In Hoofdstuk 2 lichtten we het probleem van cognitieve achteruitgang bij Parkinson toe en onderzochten we de biologische basis van cognitieve training bij mensen met Parkinson. Cognitieve beperking is een veelvoorkomend niet-motorisch symptoom bij Parkinson dat resulteert in beperkingen in aandacht, geheugen, verwerkingssnelheid, executieve functies, en visuspatiële vaardigheden. Naarmate de ziekte vordert, ontwikkelt tot wel $80 \%$ van de Parkinson populatie ontwikkelt dementie. Tot dusver is de onderliggende pathofysiologie nog deels onbekend. De identificatie van een betrouwbare biomarker voor cognitieve achteruitgang kan nieuwe kansen bieden voor cognitieve trainingen. De klinische manifestaties van de ziekte van Parkinson zijn voornamelijk gerelateerd aan een gereduceerde dopamine productie in de substantia nigra, wat leidt tot moeilijkheden in bewegen. Dopamine speelt echter ook een rol in cognitieve achteruitgang; zelfs in de vroege stadia van Parkinson kan men al problemen ervaren in verschillende cognitieve domeinen, terwijl in de latere stadia andere cognitieve domeinen volgen. De onderliggende pathofysiologie van cognitieve achteruitgang bij Parkinson is nog onduidelijk en er is nog geen betrouwbare biomarker geïdentificeerd. Verder onderzoek heeft zich gefocust op de formatie van eiwit-ophopingen, op (anders dan dopamine) neurotransmitter dysfuncties en op genetische risicofactoren. Recent is de 'dual syndroom hypothese' geïntroduceerd die enerzijds suggereert dat dopamine dysfunctie een rol speelt in planning, werkgeheugen en executieve functies en anderzijds cholinergische dysfunctie een rol speelt in visuospatiële functies en semantische vloeiendheid. Het laatstgenoemde syndroom leidt mogelijk tot een versnelde cognitieve achteruitgang en dementie [2].

Verschillende functionele MRI studies bij Parkinson beschreven dat veranderingen in functionele netwerken veroorzaakt worden door een heterogene pathofysiologie, waaronder de dopamine en cholinergische dysfuncties. Mede daardoor zijn er tussen studies grote verschillen in rusttoestand connectiviteits-patronen gevonden.

Het was ons doel om, in een groep Parkinson patiënten, de locatie en de sterkte van de connectiviteitspatronen in hersennetwerken in rusttoestand te evalueren door data uit verschillende beschikbare studies samen te voegen. Hiermee wilden we verkennen welke functionele connectiviteitsnetwerken een rol spelen in Parkinson en cognitieve achteruitgang. Ook hoopten we te verkennen wat de inzetbaarheid is van fMRI als een biomarker voor cognitieve achteruitgang bij Parkinson. Middels een literatuur zoektocht verzamelden we 1.122 
fMRI studies met Parkinson patiënten met cognitieve achteruitgang. Daaruit selecteerden we rusttoestand fMRI studies waarin Parkinson patiënten met cognitieve achteruitgang werden vergeleken met Parkinson patiënten zonder achteruitgang of gezonde proefpersonen. $\mathrm{Na}$ het filteren op specifieke analyse technieken hielden we zeventien studies over, met een gecombineerd totaal van 932 patiënten.

Om de kwaliteit van deze fMRI studies te beoordelen, ontwikkelden we criteria op basis van de voorzet van Poldrack et al. [3]. Een voxel-gebaseerde meta-analyse gecombineerd met kwalitatieve statistiek werd ingezet om neuro-beeldvormende studies te combineren met studies die enkel piek-coördinaten in een coördinaten-systeem rapporteerden (zoals Talairach of MNI).

We vonden verminderde connectiviteit bij mensen met Parkinson en cognitieve achteruitgang in onder andere het 'default mode netwerk': dit is vermoedelijk een relevant netwerk voor cognitie. Dit geeft inzicht in de verstoringen in de hersenen binnen deze doelgroep. Uiteindelijk kunnen veranderingen in functionele connectiviteit leiden tot cognitieve beperkingen bij Parkinson patiënten en derhalve een kansrijke biomarker hiervoor zijn. Desalniettemin is er nog veel onderzoekswerk nodig om deze groepsdata te vertalen naar individuele fMRI metingen.

Hoofdgedachte:

1. Een biomarker voor cognitieve achteruitgang kan leiden tot nieuwe doelwitten voor cognitieve training.

2. In mensen met Parkinson en cognitieve achteruitgang vonden we een verminderde connectiviteit in het 'default mode netwerk'.

3. Grotere studies zijn nodig om de potentiële rol van fMRI als een biomarket voor cognitieve beperkingen te verkennen.

\section{HUIDIGE KENNIS OVER COGNITIEVE TRAINING}

In Hoofdstuk 3 gingen we in op de huidige kennis en de toekomstige ontwikkelingen van cognitieve training bij Parkinson. Er is steeds meer aandacht voor niet-farmacologische behandelopties om symptomen te verlichten bij mensen met Parkinson, waaronder ergo- en fysiotherapie, beweging, dieet, niet-invasieve hersenstimulatie, etc. [4]. Parkinson patiënten hebben een vergroot risico voor een verslechterde cognitie, zowel vroeg als laat in de ziekte. Veel voorkomende cognitieve problemen zijn: cognitieve flexibiliteit, redeneren, aandacht, plannen en verbale processen [5]. De wetenschappelijke basis voor de effectiviteit van cognitieve training wordt ook steeds groter [6], waardoor dit steeds vaker als een gedegen behandeloptie wordt gezien die veilig en kosteneffectief is [7]. Een nadeel van traditionele cognitieve trainingsprogramma's is echter de eis om eenzijdige en herhalende taken uit te voeren, waardoor het programma op termijn monotoon kan worden. Dit heeft een negatieve impact op therapietrouw. Nieuwe technologische ontwikkelingen stellen ons in staat om traditionele cognitieve training te verbeteren, door aantrekkelijke elementen toe te voegen 
die ervoor kunnen zorgen dat patiënten langer blijven trainen. Zo kan de impact van de cognitieve training vergroot worden. Een voorbeeld van een technologische ontwikkeling is computer-gebaseerd cognitieve training, waar innovatieve elementen aan toegevoegd worden als een adaptieve en gepersonaliseerde trainingsinhoud. Een computer-gebaseerde training kan in de thuissituatie uitgevoerd worden en zo mogelijk preventief werken. In Hoofdstuk 7 presenteerden we de haalbaarheidsresultaten van de interim analyse van de Parkin'Play studie. Ook presenteerden we in dat hoofdstuk effectiviteitsresultaten die impliceerden dat een gegamificeerde digitale cognitieve training een positief effect heeft op cognitie bij Parkinson. In een recent review-artikel, waarin acht studies werden geanalyseerd, concludeerden de auteurs dat computer-gebaseerde cognitieve training een positief effect heeft op geheugen, verwerkingssnelheid, executieve functies en aandacht [5]. Tevens zou de toevoeging van virtual reality of kunstmatige intelligentie de effectiviteit van zulke interventies kunnen vergroten. Deze recente technologische innovaties maken het namelijk mogelijk om een flexibele digitale training vorm te geven, die schaalbaar, kosten-effectief en flexibel is, terwijl de trainingsinhoud continu aangepast wordt op de patiënt.

Dit is niet alleen relevant voor de vroege stadia van Parkinson, maar ook voor patiënten die verder in de ziekte zitten. $\mathrm{Er}$ is in het onderzoek naar computer-gebaseerde cognitieve trainingen echter meer aandacht nodig voor deze laatste doelgroep, zodat ook voor hen meer behandelopties beschikbaar worden. In Hoofdstuk $\mathbf{3}$ presenteerden we verschillende adviezen voor toekomstige gegamificeerde cognitieve trainingen voor deze doelgroep. Zo dient de gebruikersinterface overzichtelijk en eenvoudig te zijn en er dient rekening gehouden te worden met de mogelijk beperkte bewegingsmogelijkheden in de latere stadia van Parkinson. Verder dient een mechanisme toegevoegd te worden dat de trainingsinhoud automatisch aanpast op de gebruiker, eventueel ondersteund middels kunstmatige intelligentie. Een belangrijk element is het toevoegen van positieve aanmoedigingsmechanismen die direct reageren op de acties van de gebruiker. Virtual reality kan aanvullend realistische 3D omgevingen creëren, waardoor generalizatie van het trainingseffect naar het normale leven mogelijk versterkt wordt [8]. Betere en grotere studies kunnen met behulp van deze technologische ontwikkelingen ervoor zorgen dat het behandelrepertoire voor mensen met Parkinson vergroot wordt, zowel in de vroege als in de late stadia van de ziekte.

Hoofdgedachte:

1. Cognitieve training lijkt effectief te zijn bij mensen met Parkinson, maar kent ook enkele nadelen, zoals de eis om herhalende taken uit te voeren.

2. Verschillende technologische ontwikkelingen zullen in de nabije toekomst een bijdragen kunnen leveren aan computer-gebaseerde cognitieve trainingen, waaronder personalisatie, kunstmatige intelligentie en virtual reality.

3. Onze adviezen kunnen gebruikt worden om toekomstige gegamificeerde cognitieve trainingen te ontwikkelen die zowel geschikt in de vroege als de late stadia van de ziekte van Parkinson. 


\section{HET PEILEN VAN INTERESSE IN GEGAMIFICEERDE THERAPIEËN}

In Hoofdstuk 4 peilden we de interesse in gegamificeerde cognitieve trainingen bij mensen met Parkinson. Normaliter worden cognitieve trainingen uitgevoerd onder begeleiding van een professional. Daarnaast zijn de meeste computer-gebaseerde cognitieve trainingen louter kopieën van traditionele pen en papier trainingen, waardoor videogame elementen ontbreken die juist een positieve impact op therapietrouw kunnen hebben. In Hoofdstuk 4 beschreven we drie patiënten met Parkinson met verschillende leeftijden en van verschillende achtergronden. Deze patiënten gebruikten videogames, kaartspellen of daagden zichzelf in het echte leven uit om zo hun eigen cognitieve functies te trainen. Zo probeerden we aandacht te vragen voor de potentie van gegamificeerde cognitieve trainingen bij deze doelgroep. Door de wervingsresultaten van een recente cognitieve videogame training toe te voegen, concludeerden we dat ook een grotere doelgroep van Parkinson patiënten interesse heeft in zulke interventies.

De drie voorbeelden in Hoofdstuk 4 leken op vele gebieden op elkaar. Op de eerste plaats waren alle patiënten zich bewust van de achteruitgang van hun cognitieve mogelijkheden en zochten ze naar manieren om dit tegen te gaan. Op de tweede plaats voegden alle drie de patiënten een spelelement toe aan hun dagelijkse bezigheden. Op de derde plaats lieten deze patiënten zien dat ze in staat zijn om hun eigen bedachte trainingen meerdere jaren uit te voeren. Ze waren intrinsiek gemotiveerd om door te gaan met trainen doordat zij positieve effecten ervaarden van hun trainingen. De toevoeging van wervingsresultaten van de Parkin'Play studie laten verder zien dat een grote groep patiënten geïnteresseerd is in gegamificeerde training in de thuissituatie. Onze bevindingen in Hoofdstuk 4 laten zien dat de ziekte van Parkinson een aantrekkelijke doelgroep kan zijn om zulke trainingen voor te ontwikkelen.

Hoofdgedachte:

1. Sommige patiënten bedenken zelf trainingen en een grotere groep patiënten toont interesse in gegamificeerde cognitieve trainingen.

2. Personalisatie van training is ogenschijnlijk een belangrijke factor in het succes van een thuisinterventie.

3. We adviseren dat verschillende stakeholders samenwerken in de ontwikkeling van gezondheidsgames.

4. Er is behoefte aan een gestructureerde ontwikkeling en evaluatie van gegamificeerde cognitieve trainingen bij Parkinson.

\section{COGNITIEVE MOGELIJKHEDEN METEN}

In Hoofdstuk 5 werd de validatie van de MyCognition Quotient (MyCQ ${ }^{\mathrm{TM}}$ ) onderzocht: een beknopte online cognitieve test, welke in de Parkin'Play studie gebruikt werd om de training aan te passen op de cognitieve mogelijkheden van een deelnemer. Cognitieve achteruitgang komt niet enkel voor binnen een Parkinson populatie en kan derhalve ook toegepast worden binnen andere doelgroepen, waaronder psychiatrische patiënten. Het betrouwbaar meten van de cognitieve mogelijkheden is van belang in zowel klinische als onderzoeksomgevingen, aangezien het kan bijdragen aan een (vroege) diagnose, het ontwikkelen van personalisatie- 
mechanismen in interventies en kan bijdragen aan de evaluatie van behandelprogramma's. Toch zijn er enkele beperkingen aan veelgebruikte traditionele cognitieve testen: ze nemen veel tijd in beslag en behoeven aanwezigheid en begeleiding van professionals. Verder hebben onderzoekers de beschikking over een nogal ruim arsenaal aan verschillende testen en kiezen ze niet altijd dezelfde testen, wat de vergelijkbaarheid tussen studies beperkt. Er zijn al enkele gestandaardiseerde en computer-gebaseerde cognitieve testen verkrijgbaar, maar deze zijn vaak afhankelijk van relatief dure software en hardware. In Hoofdstuk 5 presenteerden we een nieuwe online cognitieve test, genaamd $\mathrm{MyCQ}^{\text {тM }}$, die speciaal ontwikkeld is om op een snelle en efficiënte manier cognitie te beoordelen (binnen 30 minuten). De test bestaat uit 10 sub-testen waarmee vijf belangrijke cognitieve domeinen beoordeeld worden: episodisch geheugen, werkgeheugen, psychomotorische snelheid, aandacht en executieve functies. De resultaten van $\mathrm{MyCQ}^{\mathrm{TM}}$ kunnen zo gebruikt worden om het verloop in cognitie te monitoren. Tevens kunnen de resultaten gebruikt worden om een cognitieve training te personaliseren. We stelden ons als doel om de validiteit van $\mathrm{MyCQ}^{\mathrm{TM}}$ te bepalen door de resultaten te vergelijken met de Cambridge Neuropsychological Automated Test Battery (CANTAB), dat ook een computer-gebaseerde cognitieve test is die vaak ingezet wordt in onderzoeken. De CANTAB neemt drie uur in beslag om te voltooien.

We includeerden 87 psychiatrische patiënten met een depressieve stoornis, een obsessieve compulsieve stoornis of schizofrenie. Alle deelnemers voltooiden de CANTAB en de MyCQ ${ }^{\mathrm{TM}}$ op een laptop in het ziekenhuis, terwijl een studente Psychologie aanwezig was om de metingen te begeleiden. We vonden een hoge correlatie tussen de $M y C Q^{T M}$ en de CANTAB totale score en vonden hoge correlaties voor de domeinen aandacht, psychomotorische snelheid, en episodisch geheugen. We vonden verder een beperkte divergente validiteit voor de domeinen executieve functies en werkgeheugen.

De $\mathrm{MyCQ}^{\mathrm{TM}}$ online cognitieve lijkt een valide en consistente methode om snel de cognitieve mogelijkheden binnen een gemengde psychiatrische doelgroep te beoordelen. Dit biedt perspectief op laagdrempelige en relatief goedkope diagnostiek in de thuissituatie. Zowel bij Parkinson als bij psychiatrische patiënten spelen dopaminerge veranderingen in de hersenen een rol in de ziekte, waarbij de dopamine waardes lager zijn en patiënten minder gevoelig worden voor beloning. Door (cognitieve) testen korter van duur te maken, en daardoor mogelijk meer belonend, kan dit wellicht bijdragen aan de betrouwbaarheid van zulke testen. Toch zijn er meer studies binnen verschillende populaties nodig om de $\mathrm{MyCQ}^{\mathrm{TM}}$ verder te valideren.

Hoofdgedachte:

1. Een psychiatrische groep patiënten is in staat om een snelle online cognitieve test uit te voeren.

2. De $M C_{C Q}{ }^{\text {TM }}$ lijkt een valide manier om cognitie te beoordelen in een psychiatrische doelgroep, maar aanvullende vergelijkingen met andere neurocognitieve testen zijn nodig in andere patiëntengroepen.

3. De MyCQ ${ }^{\mathrm{TM}}$ is een veelbelovend instrument om op een consistente manier cognitie te beoordelen, tegen relatief lage kosten. 


\section{DE EFFECTIVITEIT VAN EEN GEGAMIFICEERDE ONLINE COGNITIEVE TRAINING ONDERZOEKEN}

In Hoofstuk 6 presenteerden we het design van de Parkin'Play studie. Het doel van deze studie is het onderzoeken van de haalbaarheid en de effectiviteit van een gegamificeerde online cognitieve training bij Parkinson.

De Parkin'Play Study richtte zich op het werven van 222 Parkinsonpatiënten met een milde cognitieve stoornis voor deelname aan deze multicenter, fase-2 en open-gerandomiseerde klinische studie. Geschikte patiënten hadden Parkinson, Hoehn \& Yahr stadium I-III, waren tussen de 40 en 75 jaar oud en hadden cognitieve problemen, maar geen dementie. Deze toelatingseisen waren bewust streng, omdat we zo een homogene groep hoopten te includeren, waardoor we de kans op het vinden van een effect vergrootten. Deelnemers uit de interventiegroep ( $n=111$ ) konden verschillende cognitieve domeinen trainen via een speciaal web portaal, waarin zij een cognitieve videogame training konden volgen (AquaSnap ${ }^{\mathrm{TM}}$ ). De controlegroep $(n=111$ ) werd op een wachtlijst geplaatst en mocht later starten met trainen, in de open-label fase. Om de therapietrouw te verhogen poogden we de training te personaliseren en kon deze in de thuissituatie uitgevoerd worden. Van elke groep vroegen we 20 patiënten om ook fMRI scans te ondergaan, waardoor we mogelijk ook functionele veranderingen in de hersenen als gevolg van de training konden detecteren.

De primaire uitkomstmaat was cognitie na 12 weken training, beoordeeld door een standaard neuropsychologische testbatterij en een online cognitieve test $\left(\mathrm{MyCQ}^{\mathrm{TM}}\right)$. We berekenden een totaalscore voor cognitie samengesteld uit alle sub-test Z-scores. Secundaire uitkomstmaten, waaronder therapietrouw, biologische maten en verschillende motorische en niet-motorische testen, werden afgenomen tot 24 weken na de start van de training.

\section{THERAPIETROUW BIJ EEN GEGAMIFICEERDE COGNITIEVE TRAINING}

Gamificatie en spelelementen kunnen mogelijk voordelen bieden ten opzichte van traditionele cognitieve training, onder andere op het gebied van aantrekkelijkheid en therapietrouw [9]. Zo zullen mogelijk meer patiënten langer doortrainen, waardoor het trainingseffect sterker kan zijn en langer aan kan houden. Tot dusver is er echter nog geen onderzoek gedaan naar gegamificeerde cognitieve training bij mensen met Parkinsonpatiënten. In Hoofdstuk 7 presenteerden we de resultaten van een interim analyse naar de haalbaarheid van zo'n training.

We voerden een gerandomiseerd onderzoek uit waarbij Parkinsonpatiënten met een milde cognitieve stoornis willekeurig werden toegewezen aan een gegamificeerde online cognitieve training die zij 12 weken volgden. Deelnemers werd gevraagd om thuis, driemaal per week gedurende 30 minuten per sessie te trainen, terwijl de anderen aan de wachtlijst controlegroep werden toegewezen. Vóór deelname, en in week 12 en 14 werd een neuropsychologische cognitieve testbatterij afgenomen in het ziekenhuis. Na week 12 mochten deelnemers uit beide groepen de training vrijwillig volgen. 
We includeerden 41 vergelijkbare patiënten in deze interim analyse (21 interventiegroep; 20 controlegroep). In de interventiegroep trainde $69 \%$ van de deelnemers meer dan $50 \%$ van de gevraagde trainingsminuten en $81 \%$ van de deelnemers ging vrijwillig door met trainen na 12 weken. $\mathrm{Na} 24$ weken beoordeelde $87,5 \%$ van de deelnemers het spel positief.

De resultaten van de interim analyse laten zien dat het haalbaar is voor Parkinsonpatiënten met een milde cognitieve stoornis om een gegamificeerde cognitieve training te volgen. Ook lieten ze een acceptabele therapietrouw zien; vele trainingssessies werden uitgevoerd, zelfs na vele weken. Het relatief hoge aandeel patiënten in de interventiegroep dat er vrijwillig voor koos om door te trainen na 12 weken geeft aan dat de aangeboden interventie voldoende aantrekkelijk was. Desondanks kan de therapietrouw nog verbeterd worden, en actieve coaching op afstand kan hier een rol in spelen. Het rapporteren van therapietrouw gebeurt niet vaak in studies met computer-gebaseerde cognitieve training, ondanks dat wij dat belangrijk achten. In een andere studie onder patiënten met multiple sclerose (MS) vonden we echter toch vergelijkbare resultaten met betrekking tot therapietrouw [10].

De effectiviteitsmaat toonde een interessante trend: de totale cognitiescore verbeterde na 24 weken trainen. Deze studie bevatte echter een te kleine groep patiënten om effectiviteit aan te kunnen tonen.

\section{Hoofdgedachte:}

1. Het is haalbaar voor Parkinsonpatiënten om een gegamificeerde online cognitieve training gedurende vele weken thuis te volgen.

2. In toekomstige trainingen moeten verschillende verbeteringen aangebracht worden om therapietrouw te verbeteren, zoals het toevoegen van ondersteuning en coaching op afstand.

3. Het is te vroeg om vast te stellen dat deze gegamificeerde training een positief effect heeft op cognitie. 


\section{WAT WE GELEERD HEBBEN}

In dit proefschrift probeerden we de haalbaarheid en effectiviteit te evalueren van een gegamificeerde online cognitieve training bij mensen met Parkinson. Het project bestond uit drie fases:

Fase A De ontwikkeling van een gegamificeerde cognitieve training die in de thuissituatie uitgevoerd kon worden, in samenwerking met patiënten en MyCognition Ltd. Het doel was om een wetenschappelijk onderbouwd trainingsprogramma te maken dat zowel aantrekkelijk als effectief was.

We leerden In samenwerking met MyCognition Ltd. (Londen, VK) en een gameontwikkelaar maakten we de gegamificeerde cognitieve training AquaSnap. De conceptversie werd gepresenteerd aan Parkinsonpatiënten in Maastricht en hun feedback werd ingezet om het product te verbeteren. Aanvullend vonden we het belangrijk om onderzoek te doen naar de biologische basis van cognitieve achteruitgang en ontdekten verminderde connectiviteit in hersendelen die onder andere een rol spelen in cognitie (default mode netwerk). Ook deden we onderzoek naar de interesse vanuit de doelgroep in zulke gegamificeerde cognitieve trainingen en vonden verschillende patiënten die in hun eigen leven al zelf trainingen in spelvorm hadden ontwikkeld. Bovendien concludeerden we uit de wervingscijfers van de Parkin'Play studie dat veel Parkinsonpatiënten interesse hebben in dit soort trainingen.

Fase B-1 Het uitvoeren van een pilot studie naar de haalbaarheid van een gegamificeerde cognitieve training bij mensen met Parkinson en cognitieve beperkingen, waarbij deelnemers tot 24 weken een thuistraining konden volgen.

We leerden In onze studie mochten de deelnemers de videogame training thuis volgen. We concludeerden dat het uitvoeren van dit soort trainingen haalbaar is voor mensen met Parkinson. Sommige factoren hebben de therapietrouw mogelijk verlaagd, waaronder de geringe variatie in trainingsinhoud en het ontbreken van coaching op afstand.

Fase B-2 Het uitvoeren van een pilot studie naar (voorlopige) effecten van een gegamificeerde cognitieve training. Een actieve interventiegroep werd vergeleken met een wachtlijst controlegroep na 12 weken training (gericht op de cognitieve domeinen: werkgeheugen, episodisch geheugen, aandacht, psychomotorische snelheid en executieve functies).

We leerden We stellen voorzichtig dat onze training een bescheiden positief effect had op de totale cognitie, maar dit effect is slechts net significant bij deze groepsgrootte $(p=0.49)$. Dit is echter bemoedigend voor een toekomstige grotere studie, welke nodig zijn om een definitief effect aan te tonen. 
Fase C Het uitvoeren van een grotere studie naar de effectiviteit van een (verbeterde versie van een) gegamificeerd cognitief trainingsprogramma bij mensen met Parkinson, waarin alle lessen uit de vorige fases worden opgenomen in een verbeterd trainingsprogramma en een sterker studie design.

We leerden Deze fase hebben we (nog) niet bereikt. Daardoor kunnen we niet definitief concluderen dat gegamificeerde cognitieve training een positief effect heeft op cognitie bij mensen met Parkinson. We adviseren onderzoekers om verschillende elementen toe te voegen aan toekomstige gegamificeerde interventie studies, waaronder: meer variatie in spelinhoud, coaching op afstand, een "blinde" beoordelaar tijdens de metingen en een actieve controlegroep.

\section{REFERENCES}

1. Cools R. Dopaminergic modulation of cognitive function-implications for L-DOPA treatment in Parkinson's disease. Neurosci Biobehav Rev 2006;30(1):1-23. PMID: 15935475

2. Painous C, Marti MJ. Cognitive Impairment in Parkinson's Disease: What We Know so Far. JPRLS Dove Press; 2020;Volume 10:7-17.

3. Poldrack RA, Fletcher PC, Henson RN, Worsley KJ, Brett M, Nichols TE. Guidelines for reporting an fMRI study. Neurolmage 2008 Apr 1;40(2):409-414. PMID: 18191585

4. de Vries NM, Nonnekes J, Bloem BR. Toward affordable falls prevention in Parkinson's disease. Mov. Disord. John Wiley \& Sons, Ltd; 2016 Jan;31(1):3-6. PMID: 26660664

5. Nousia A, Martzoukou M, Tsouris Z, Siokas V, Aloizou A-M, Liampas I, et al. The Beneficial Effects of Computer-Based Cognitive Training in Parkinson's Disease: A Systematic Review. Arch Clin Neuropsychol 2020 Jan 14;13(4):217. PMID: 31942917

6. Alzahrani H, Venneri A. Cognitive Rehabilitation in Parkinson's Disease: A Systematic Review. Journal of Parkinson's disease IOS Press; 2018;8(2):233-245. PMID: 29614698

7. Leung IHK, Walton CC, Hallock H, Lewis SJG, Valenzuela M, LampitA. Cognitive training in Parkinson disease: A systematic review and meta-analysis. Neurology 2015 Oct30;85(21):1843-1851. PMID: 26519540

8. Larson EB, Feigon M, Gagliardo P, Dvorkin AY. Virtual reality and cognitive rehabilitation: A review of current outcome research. NeuroRehabilitation IOS Press; 2014 Jan 1;34(4):759-772.

9. van de Weijer SC, Kuijf ML, de Vries NM, Bloem BR, Duits AA. Do-It-Yourself Gamified Cognitive Training: Viewpoint. JMIR Serious Games 2019 May 7;7(2):e12130. PMID: 31066713

10. Shatil E, Metzer A, Horvitz O, MillerA. Home-based personalized cognitive training in MS patients: a study of adherence and cognitive performance. NeuroRehabilitation 2010;26(2):143-153. PMID: 20203380 


\section{DISCUSSION \& INTERPRETATION}

\section{FEASIBLE FOR EVERYONE}

Based on this thesis, we conclude that gamification of home-based cognitive training holds promise as a feasible approach in PD. Encouragingly, it has been found that gamified interventions are feasible in other populations as well [1]. In Chapter 4 and Chapter 7, we found that PD patients are interested in both exercising a gamified intervention and joining a study on gamified cognitive training. The cases presented in Chapter $\mathbf{4}$ experienced reward and self-efficacy when they performed their gamified intervention, which may lead to increased resilience to difficulties or failure [2]. All three patients continued their self-invented training over longer periods of time (many years) and, more importantly, they all perceived subjective cognitive benefits. In Chapter 7, we showed that PD patients were able to perform such an intervention unsupervised, on a regular basis in their homes. We also saw that some patients showed high adherence to the gamified cognitive training, whereas others did not even start training. We believe that initial interest in an intervention is an important first step for sound feasibility, but that there is not just one intervention that fits all patients. If patients are unattracted to a specific type of intervention, they are unlikely to use it. Of course, they might use such an intervention initially, but the continuance will be compromised, among other things due to limited intrinsic motivation [3]. Cognitive training is not only suitable or effective in PD-MCl patients. As we showed in Chapter $\mathbf{2}$, fMRI research also shows similarities between patients with different cognitive profiles. Hence, patients in the pre-stages of $\mathrm{MCl}$ or patients who have progressed to dementia should also be allowed to follow these therapies.

We do not believe that gamified cognitive training is suitable for every patient, similar to not all sports being attractive to those who like to play golf. This may explain why some patients in our study showed excellent adherence, but others did not, despite consenting to the study. Therefore, we recommend the creation of a menu or repertoire of cognitive training interventions of which a patient can choose from, so that every patient can follow a therapy that fits their personal preferences and/or may switch between interventions as long as these interventions target the areas that require training for the particular individual. Yet, other facilitators and barriers for treatment adherence have been found in the literature as well. Among those are patient-centered factors (e.g. attitudes and beliefs towards the therapy, history of good compliance, technological skills), therapy-related factors (e.g. treatment complexity, duration of the treatment period, degree of behavioral change required), healthcare system factors (e.g. accessibility, unsatisfactory clinic visits), social and economic factors (e.g. inability to take time off work to exercise the treatment, social support, cost of the treatment), and disease factors (e.g. severity of the disease) [4]. Especially therapy-related factors and healthcare related factors should be addressed when developing new interventions. For example, the therapies can be created in such a way that they are technologically easy to exercise and attractive for longer durations. Additionally, providing adequate therapy knowledge in terms of evidence for therapy efficacy may also add to 
improved beliefs towards the therapy. Adherence facilitators should be added while adherence barriers should be prevented while developing new therapies. Moreover, interventions can be developed to be low-cost and accessible and accessible in the home situation. Home-based health care delivery is especially relevant in the midsts of health threats like COVID-19, because it reduces the risk of spreading the virusses to vulnerable patients and simultaneously provides to address health issues, such as cognitive decline.

Feasible for everyone? Elements to consider:

- There is not just one intervention that fits all patients.

- Clinicians, patient and caregivers should jointly consider a variety of interventions, so that every patient can follow a therapy that fits their personal preferences.

- Facilitators and barriers for treatment adherence should be addressed while developing new therapies. These factors are patient-related, therapy-related, healthcare related, and disease-related.

\section{TECHNOLOGY USABILITY FOR ELDERLY}

Our research focused on an elderly population, on average about 65 years old. In 2017, 67\% of the American elderly population had internet at home [5]. This percentage resembles the number of elderlies that had our bought this technology, but it does not consider whether they are actually using the technology after buying it. A recent qualitative study suggest that barriers to technology use among elderly include low technology literacy, physical challenges, and top-down design processes [6]. Many interviewed elderlies expressed feelings of frustration when using technology, which resulted in decreased self-confidence and decreased motivation to pursue using the technology. When we are designing a computer-based and home-based intervention, we also need to ascertain the requirements of the target group to be able to use such technologies.

In our research, we saw that there definitely is an interest of elderly people in technological interventions. More importantly, this group is also willing to contribute to the design of technologies that would facilitate aging independently [6]. When designing the AquaSnap intervention, several patients participated during the review process, which gave valuable insights into the usability of the setup. I believe that user-centered design strategies, in which the end-user/patient plays a role in the development of the intervention, could increase the adoption rate of such interventions and improve the technological literacy and motivation of that target audience. In our trial, although all subjects were motivated initially to start the treatment, we experienced that technical difficulties occasionally prevented the subjects from being able to play the game, thereby decreasing their motivation. We expect that this has influenced our adherence results in a negative way, and it would be interesting to research the updated version of our gamified cognitive training for improved adherence.

We need to strongly focus on so-called "system usability" in user-centered design strategy as well. The digital technology-based home care products should just work, without compromises. 
It can be frustrating for older adults to use a technology that does not work intuitively or perhaps only works when specific requirements are met, such as using a specific browser on Windows computers. In Chapter $\mathbf{7}$, we indeed saw that these technical difficulties even caused some subjects to drop out of home-based treatments. A technological intervention should for example be supported across platforms (i.e. various versions of Windows, MacOS, IOS, Android) and it should contain both online and offline modes to increase usability for elderly with a (temporary) lack of internet connection. When designing a technological intervention for elderly populations, our requirements as presented in Chapter $\mathbf{1}$ and Chapter $\mathbf{2}$ can be used to make the intervention as user-friendly and as intuitive as possible. Some important recommendations to note here are presented in the following box.

Technology usability in interventions. Elements to consider:

- $\quad$ Provide clear instructions and perhaps a digital walkthrough.

- Set reachable goals and objectives.

- Present neutral and appropriate feedback, without negative remarks.

- Slowly introduce cognitively demanding elements.

- Design a graphically appealing gameplay for elderly that and can be adjusted (in terms of contrast and size).

- All parties involved in intervention design should collaborate to develop motivating and targeted interventions for specific elderly target groups.

- New treatment options should incorporate thorough implementation and remotesupport procedures in order to increase the likelihood of adoption success.

Unfortunately, in many cases, there is too little or no input from patients in designing a new technological intervention. This top-down design process may result in mismatches between the needs of those patients and the technologies that are used. Thus, we advise researchers, health professionals, patients, and the industry to collaborate with patients, being the primary end-users, to develop motivating and targeted interventions for specific elderly audiences. Effective interventions are those that prioritize the needs and wishes of patients, focus on general acceptance of potential users, and create effective preconditions for its adoption. This knowledge is becoming more mainstream in recent years (e.g. participatory healthcare), resulting in initiatives that aim to connect researchers, patients and doctors in order to facilitate innovations. An example of one such an initiative is ParkinsonNext,which is a web-based platform where patients interested in research are connected to researchers seeking participation of patients (www.ParkinsonNEXT.nl).

Perhaps an even more important factor in the success of a new digital intervention is the implementation and support process. New treatment options should incorporate thorough implementation procedures and frequent, remote-support contact moments in order to increase the likelihood of adoption success and the continuation of the intervention. Among others, this includes implementation training, implementation documentation, a dedicated implementation project coordinator, and frequent contact moments between the participant and the hospital staff. Currently, Dutch coaches are already being trained to effectively 
implement cognitive training programs and deliver the types of support mentioned above to patients in order to stimulate adherence.

\section{FUN OR SERIOUS}

It definitely is a challenge to create a fun intervention or game, and the success of a game does not only depend on how well a game is designed. Why is a game like Candy Crush immensely popular, but are other presumably more sophisticated games not?

There is an extensive literature database available on game development and factors that influence the attractiveness and "fun-ness" of a game. The book "A Theory of Fun: for Game Design" [7] explains that most successful games are built upon the same elements, of which some have been mentioned throughout this thesis (e.g. reward). In Chapter 7, we saw that our study subjects experienced boredom at a certain point during the trial. This was partly due to the limited number of cognitive trails that were built into the initial version of AquaSnap: after training more than 18 hours, no new trials were added to the game. Other reasons were the lack of introducing new levels and new worlds within the game. We think that introducing a large variety of attractive exercises can prevent boredom and abandonment. Whereas we earlier proposed the professional to select a variety of interventions from with the patient could choose (more therapies with various themes), each intervention should additionally contain various exercises and various levels that make it more attractive and fun for longer periods of time, in order to ensure the user stays engaged and motivated to use it for as long as necessary. In short: an effective gamified intervention teaches all new competences it has to offer before the player stops playing and within the updated version of AquaSnap, many new variances have been added to address these issues.

Nonetheless, attractiveness or fun factor may not be the only essential elements for the success of a gamified intervention. After having conducted several gaming studies in PD, we feel there needs to be a clear balance between the fun factor in and the goal of the intervention. Therapy designers aim to make a training a more bearable activity, because they hope that this results in increased health benefits in the long run. At the same time, it should be made clear to patients that the most important goal of performing a gamified intervention is essentially not the fun factor, but rather is it the willingness to improve health and the necessity they feel to do so (i.e. intrinsic motivation). We frequently compare cognitive training with physical workouts in the gym: the workouts themselves are neither fun or easy, but a combination of challenges (complete $4 \times 10$ push-ups) and clear goals (lose weight or increase fitness on the long run) results in a cocktail for success. Following that analogy, it is obvious that a many people fail to regularly visit the gym due to dislike for the activity, probably because the goals are unclear, and results are booked slowly (which may have been promised differently at the outset). For persons with PD, this is even more complex, since reward anticipation and reward processing are disturbed in PD patients due to a combination of dopaminergic denervation and, for some, the adverse effects of dopaminergic medication. Interventions with fast-reward features are 
therefore more likely to be complied to as compared to interventions with future-reward features. Improving health is hard work, and this needs to be communicated clearly to patients.

Communication and education are factors that can influence a patient's motivation and are therefore facilitators for adherence to therapies. When a patient believes that the disease is posing severe consequences for his health and when he believes that the proposed therapy benefits the disease progression, he is more likely to be intrinsically motivated to adhere to the treatment [4]. In contrast, misconceptions or flawed beliefs about the disease and the treatment will negatively affect adherence. Hence, healthcare professionals play an important role in educating the patients about the disease and the treatment, while researchers play an important role in coming up with evidence for the treatment's efficacy.

Fun or serious? Elements to consider:

- An intervention should contain various exercises and various levels that make it more attractive for longer periods of time.

- Arguably, the most important goal of performing a gamified intervention is not the fun factor, is it the willingness to improve.

- Communication and education about the disease and the treatment are facilitators for adherence, and both healthcare professionals and researchers play an important role in this.

\section{OBJECTIVE VERSUS FUNCTIONAL IMPROVEMENTS}

Whenever the discussion of the effectiveness of cognitive training comes up, there is always the question what can be expected in terms of post-training cognitive functions. First, in order to determine the effectiveness of a cognitive training, it is important to understand what types of assessments are available. There is a broad range of standard cognitive tests available that have proven to be reliable, valid, and in measuring specific aspects of cognitive functioning. In recent years, criteria for PD-MCl and PD-dementia have been compiled, which entail the conduction of an array of standard and valid cognitive tests. To meet the criterium of PD-MCl, a patient needs to be diagnosed with PD and cognitive disturbances need to be present, but the patients are still able to function autonomously [8]. To meet the criterium of PD-dementia, a patient meets all earlier criteria, but the cognitive decline also severely interferes with normal functioning in daily life [9]. Although these cognitive tests are timeconsuming, they are currently essential in establishing $\mathrm{MCl}$ or dementia at baseline in a patient. For treatment evaluation or progression monitoring, however, a brief screening is preferred and supports the development of cognitive assessment alternatives. Widely used alternatives are the Mini Mental State Exam and Montreal Cognitive Assessment, which assess multiple cognitive domains, but these are still pen-and-paper assessments that require professional involvement to complete. In our opinion, future studies should focus on creating a brief and simple standardized core outcome set for cognitive assessments that is focused on improving outcome reporting, thereby comparability and replicability between studies, and this is supported by other researchers [10]. Additionally, it is important to assess mood, behavioral 
issues, functional abilities, and medical or surgical treatments, as they can affect cognition in PD as well [11], but these assessments do come at the cost additional time.

A web-based cognitive assessment has potential benefits over traditional cognitive assessments, since the former can be standardized, completed in the home situation, and it therefore does not require specialist supervision, preventing time consuming and costly assessments that are confined to a clinical setting. The MyCQ was developed to screen the broad cognitive status of patients in user-friendly and quick manner, which could then be used to adjust the difficulty level and speed of the intervention to each patient's abilities and specific deficits (i.e. tailored individually). We found that the MyCQ output was comparable to the Cambridge Neuropsychological Automated Test Battery, which is an extensive computerbased cognitive assessment [12]. Our research did not investigate the reliability of cognitive assessments in the home situation, and it is still unclear how potential confounders, such as distractions, partners' assistance, or substance use, may affect the reliability and validity of the results. Yet, the MyCQ provides an opportunity for home-based assessment, which on the one hand might be less burdensome for patients and cost and time efficient, and which might function as a more real-time cognitive score on the other hand. In this way, it could also be used as an adaptation mechanism within the (gamified) and home-based cognitive training.

Assessing cognition using an objective cognitive test is one way to investigate the effectiveness of a cognitive training, but an alternative method should be to look at the subjective and functional improvements that are meaningful for patients (e.g. quality of life, activities of daily living) as well, in which respectively the subjective views of the patients and the benefits of engaging in activities are examined. The Institute of Medicine released a report called "Cognitive Aging" and proposed new criteria for the evaluation of cognitive training programs [13]. These criteria include the establishment of efficacy through comparisons of active control groups, post training skill retention, replicability across studies, and the transferability of the effect to relevant real-world situations and tasks. For patients, it would be most beneficial when functional benefits can be achieved. We also think that subjective cognition (when a patient subjectively feels better) adds to the success experience of patients. An emphasis on these new criteria should be added to future studies. 
Objective versus functional improvements. Elements to consider:

- Objective criteria are mostly used in research and form an important gold standard of assessing cognitive function.

- Web-based cognitive screenings could speed up the process of cognitive treatment evaluations or cognitive progression monitoring.

- Home-based cognitive screening allow for frequent monitoring without the need for a clinical setting.

- To meet the criterium of PD-MCl, a patient needs to be diagnosed with PD and cognitive disturbances need to be present, but the patients are still able to function autonomously. To meet the criterium of PD-dementia, a patient meets all earlier criteria, but the cognitive decline also severely interferes with normal functioning in daily life.

- Subjective and functional cognition should be considered essential in assessing the efficacy of cognitive training programs.

\section{LIMITATIONS}

There are a few shortcomings to the research presented in this thesis. Firstly, including a passive waiting list as the control arm resulted in several limitations. We cannot conclude that gamification of cognitive training results in increased adherence to the intervention, because we did not include an active control arm receiving standard of care (a regular, nongamified cognitive training). Future studies could include an additional control arm receiving standard of care to investigate the benefits of gamified interventions. Also, because we did not include an active control arm receiving a regular game without cognitive training trials, we cannot conclude that a possible effect on cognition is specific for this gamified cognitive training intervention or that this effect would appear in other activities as well. In another study, for example, it was found that playing Super Mario was more beneficial as compared to a specifically designed cognitive training game [14]. Nevertheless, we did deliberately add a passive waitlist-control group, because we wanted to investigate whether a gamified cognitive training intervention has any effect on cognition at all as compared to no treatment (which is the reality in current usual care). We would not be able to establish this if we provided the control group with an (sham) intervention.

Secondly, both researchers and patients were unblinded in our main study. This was a practical limitation that was introduced because we (1) did not provide a sham intervention to the control group and (2) we did not have enough research assistants to do either the assessments or deliver the cognitive training instructions and technical support. Hence, both the subjects and the investigator were aware of the randomization of the subject and this potentially influenced the results. The results of double blinded RCT's are - justifiably - considered much more reliable as compared to non-blinded studies. Therefore, future gamified cognitive training trials should add sham interventions and enable reliable and blinded assessments. 
Thirdly, a limitation of this study was the limited working capacity to perform such a large trial. We aimed at including 222 subjects but including such a large number subjects via their own treating neurologist proved to be quite a challenge, and it took almost 24 months to include only $18.5 \%$ of the projected sample size. We saw a promising spike in registrations after sending out two newsletters through the national Parkinson's Disease association (Parkinson Vereniging) and the ParkinsonNext service (www.ParkinsonNEXT.nl). We were unfortunately unable to complete the full study, due to financial limitations.

\section{FUTURE OUTLOOK}

In summary, gamification of cognitive training holds promise as a feasible approach in persons with PD. Larger trials are needed to establish efficacy of gamified home-based cognitive training in PD. A two-armed double-blind design that compares a coach-guided, online cognitive training game with care as usual is required to investigate the added benefit of gamification as compared to standard of care (which typically involves no cognitive training).

As discussed in our limitations, for a new intervention to be clinically relevant, it should be at least as effective as the standard care. Based on our own research presented in this thesis, it remains to be seen whether gamified elements are a beneficial intervention in PD. This has not been investigated for cognitive training yet, but gamified physical exercise-based interventions found that gamified exercise-based interventions are relevant alternatives to rehabilitation at home, because the effectiveness of these interventions was at least comparable to traditional therapy or usual care [15]. Unexpectedly, however, they also found fairly high attrition rates and reported reasons for participants declined or discontinued the intervention: (1) technological issues, such as lack of internet connection, (2) discouragements when confronted with technological devices, (3) lack of customization and negative feedback in commercial games, and (4) childish or juvenile graphic design of the games. These reasons for non-adherence can be particularly informative for future non-exercise-based gamified interventions as well.

On another note, the addition of physical exercise to cognitive interventions has been investigated in PD as well. In a recent study among 125 PD patients, it was found that a highintensity aerobic exercise was able to attenuate off-state MDS-UPDRS motor signs in PD, but the effects on cognition were not clinically relevant [16]. After 6 weeks to 18 months of aerobic exercise, a large study encompassing 2,049 PD participants found cognitive improvements in processing speed, executive function, attention, and memory [17]. A combined treatment of cognitive and physical training seems a valid strategy in PD as well. A potential risk of this combined strategy is the occurrence of physical injury, but an important additional advantage is transfer of effects between cognitive and motor skills, for example, cognitive training's beneficial effects in physical symptoms such as freezing of gait [18]. Exergames are a form of therapy in which exercise and gaming are combined into a training in which the patient must conduct physical movements while performing cognitive exercises. Exergames show exciting potential for both physical and cognitive rehabilitation in PD [19]. 
After analyzing the results of our Parkin'Play pilot study, we feel that several factors negatively influenced adherence in our study, among which are technological issues, a lack of a variety of exercises and a lack of supervision while training at home. Many of these could be prevented in the future, and the former two factors have been discussed in this chapter already.

In the future, home-based intervention studies could benefit from a blended approach including a personalized training calendar and remote supervision to the intervention in order to set goals, to track progress of the participant and to add accountability. As suggested in Chapter 7, in our work with PD patients, we reach lower adherence scores when we allow patients to exercise the intervention whenever it suits them. In future intervention studies, we plan to sit down with patients to create a personalized training calendar that fits their personal schedule. Additionally, we aim to instruct them to inform us when they deviate from the agreed training times. We also plan to map individual facilitators and barriers to come up with a personalized approach. In addition to the personalized training calendar, we also plan regular calls with remote research assistants to follow-up on the subjects' progress. The success of remote supervision in interventions in PD has recently been published [16]. These researchers found a significant effect of home-based and gamified exercise training in PD, and excellent adherence due to remote supervision. In both my academic and clinical experience, patients appeared to be more motivated when a person (research assistant) both called and emailed them on a frequent basis, which generally resulted in better adherence in using the homebased eHealth products. In future interventions, this system could be further enhanced by automating it within the gaming platform, for example via integrations with digital calendars and or automatic e-mail reminders. By adding remote supervision to interventions, we hope to reach a state in which the patient's accountability increases, thereby feeling more responsibility for his own acts and behaviors. Nevertheless, feelings of failure or frustration with the training might at some point become inevitable during training, and teaching the patients strategies to cope with these might result in less drop-out. Consequently, these aspects should increase adherence to home-based health care and ultimately benefit health outcomes.

Several new innovations are increasingly being introduced in videogames in the last decennium. Current cognitive assessment methods are aimed at assessing a patient's cognitive performance in a clinical setting. In the future, the cognitive performance of a patient could be assessed by measuring its performance in the home situation in between game sessions or while playing the game. This data can be used to tailor the gameplay in real time to the abilities and requirements of the player.

More knowledge of how humans learn and transfer skills into real life is required to develop more effective virtual interventions. Experimental paradigms with simple tasks in experimental contexts, such as remembering a certain number of items, are usually at the base of developing of new interventions. When patients exercise such an experimental intervention in controlled environments, it remains to be seen whether new skills are transferred to real world skills or not. Especially in cognitive training, there is a large body of critique on this topic. Hence, 
another particularly interesting innovation for cognitive training is virtual reality. Using virtual reality, patients can be introduced in virtual real-world settings which hopefully results in transfer of complex skills, while still being in a relatively experimental or controlled setting. The precision at which virtual reality can control such a setting, and the subjects within it, exceeds the possibilities of real-world physical environments. Now, patients can walk through a virtual store and collect the items they had to remember. VR is found to improve patients' motivation as well, increasing the potential benefits of the intervention. The V-TIME project by Mirelman et al. is a successful example of such a virtual reality intervention [20] with benefits on falls reduction by VR treadmill training as compared to non-VR treadmill training.

Artificial intelligence (Al) algorithms are used in games to generate responsive gameplay, but a distinction should be made between "game Al" and "academic Al". Experts in the field of videogames state that game Al includes relatively - in the Al world - simple techniques that present a limited set of predetermined responses to a limited set of predetermined inputs by the player. Academic Al is much more complex and facilitates computer learning, in a way that the games can create content themselves based on inputs that are not necessarily predefined. Academic Al results in clever human-like systems and is in my opinion particularly interesting for the future of videogames and gamified interventions. Using Al, the game settings automatically tailor to the interaction of the user with the game, mapping those interactions to levels of cognitive states and adjusting the gameplay in real-time, theoretically resulting in more efficient therapies. A sub-group in Al, data mining, involves discovering patterns in large data sets, which is done using machine learning. Large datasets from patients/players, perhaps with the help of new types of studies [21], can then be analyzed (in real time) and grouped into comprehensible pieces of information. Most interestingly, this could result in the creation of patient profiles that predict future behavior. When a gamified intervention can read personal patient profiles, it can then tailor the content specifically to that patient, which then optimizes adherence and health benefits. These virtual reality and AI techniques are now being introduced in regular videogames and should be considered closely by serious game designers.

Future improvements for cognitive training. Elements to consider:

- Use articifial intelligent algorhitms to personalize the intervention

- Increase the number of variations in gamification elements

- $\quad$ Provide (remote) proactive support staff for patients, that assist in designing a personalized training calendar, monitor and give feedback on progression, give advise with regard to training location and frequency, set and monitor training goals, etc.

- Add elements that could promote transfer to real life, such as virtual reality

- Educate the patient on the training rationale, the expected effects, and strategies for coping with failure or frustration

As long as there is no surgical or medical treatment for cognitive decline, we are confident that a new cognitive therapy can be created that strikes the ultimate balance between gamification, personalization, attractiveness, precision and efficiency. Future studies should ascertain the 
requirements of successfully delivering home-based interventions. In addition, future studies should investigate the optimal levels, dosage, duration and timing of cognitive training in PD. We think that in the human brain, there is room to maintain cognition and autonomy for longer periods of time if training is started as soon as the first symptoms arise, or preferably even sooner. Due to functional brain plasticity, we also believe that the human brain is physically capable of shifting functions to other brain regions or circuitries, thereby helping to maintain cognitive function. We doubt whether cognitive training can ever restore cognition to preclinical levels when it is already significantly declined, but progression to dementia is not inevitable [11] and this should be a target on the horizon.

Further innovations will improve cognitive training elements by making it smarter (Al), more tailored (gamification) and potentially more generalizable to real life (exercise and/or VR), which usually is a disadvantage to functional cognitive training. In the ideal scenario, cognitive functions can be trained for longer periods of time by an array of cognitive therapies that transfer improvements into a real-world setting, so that a patient experiences the true success of the therapy.

One day, innovations will enable patients to have various home-based interventions to be personalized, attractive, precise, and efficient, all at once.

\section{POTENTIAL IMPACT OF THIS THESIS}

\section{SCIENTIFIC RELEVANCE}

Digital technology in health care empowers healthcare professionals to deliver care to patients' homes, thereby making them less dependent on hospitals or nursing homes. The research presented in this thesis adds to the knowledge of both the feasibility as well as the efficacy of digital technology-enabled cognitive training in an elderly population, with a focus on gamification. In the scientific community, researchers can benefit from our feasibility results to improve adherence in future studies. First, our results suggest that it might be important for researchers to assist research subjects in planning their intervention activities, by creating a personalized training schedule. Second, elderly may require more time to familiarize themselves with digital interventions and this process might initially be in need of guided training sessions. Third, the addition of pro-active remote support, in which subjects are contacted on a frequent basis about their therapy progression, may further enhance adherence to the treatment. By following these guidelines, researchers might additionally prevent dropout due to a mismatch between research requirements and patients' capacities to follow those expectations.

In the ideal scenario, cognitive functions can be trained for longer periods of time by an array of cognitive therapies that transfer improvements into a real-world setting, so that a patient experiences the true success of the therapy. Gamification is all about engagement. Patients 
that are engaged to a therapy are first and foremost more likely to complete the treatment plan, but they also have a greater shot at experiencing the beneficial effects introduced by the therapy, such as living independently at home for longer. From a scientific perspective though, there still is a lot to learn about specific elements in gamification that promote the attainment of effective skills the most.

Our resting-state fMRI meta-analysis provides additional evidence for the association between cognitive decline and reduced connectivity in the default node network in PD, this was suggested by other researchers as well (Chapter 2 ). Therefore, our results may provide additional support for continued research in this area, and this potentially results in a future biomarker for cognitive decline in PD. Finding a valid biomarker for cognitive decline could provide new opportunities for early detection of cognitive decline, and consequently may also yield timely intervention of this decline, which is suggested to be more beneficial as compared to intervention later in the disease. Gained knowledge in this relatively new territory may also provide insights in new biological targets for cognitive rehabilitation, which could then be targeted with improved-adherence interventions.

\section{POTENTIAL FOR HOME-BASED CARE}

In addition to its relevance in research, the gained knowledge in this thesis can be particularly relevant for both caregivers and health care in general. Even if patients are receiving interventions without gamified elements, caregivers should consider the adherence-promoting strategies that were presented in this thesis, such as active (human) support throughout the use of the platform as well as tailoring of the platform to the user's competences, as assessed by a digital assessment (Chapter $\mathbf{5}$ ).

In addition, the vast majority of health care is still delivered in a traditional hospital setting. The cost of delivering certain types of health care to the patients' homes could be significantly lower. Due to home-based health care, patients can start better managing their own health and wellbeing. Digital home-based health care can also act as a prevention measure to hospital or nursing home admissions and limit readmissions to hospitals or nursing homes [22]. Moreover, in the midst of cross-border health threats like (corona)viruses, hospitals and nursing homes can become high risk settings for those who are more vulnerable to infections. Hereby, digital and home-based interventions are more indispensable than ever by decreasing the risk of infections. During pandemics, digital tech-enabled home care benefits hospital resources management in a couple of ways. Firstly, less staff capacity is required to deliver care to patients in a hospital setting. Secondly, less infections mean more capacity in the nursing wards and Intensive Care units for those who need it. Thirdly, gamification elements can be used to enhance knowledge and application of certain infection prevention procedures by caregivers as well as patients [23].

Further, in health care, gamification could be added as compliance-increasing elements to other target groups and contexts, for example, therapies for psychological disorders, 
physical training, or even medication management. Yet, can our research, and gamification in particular, serve a purpose beyond the context of health care? Gamification can have an impact in other contexts as well, and I think both education and business can especially benefit from lessons learned here.

\section{GAMIFICATION IN EDUCATION}

Our current educational system contains some flaws, in my opinion, that are potentially at the basis of increasing disinterest and boredome among students. Firstly, one of those flaws is group size. Instead of tailoring courses to the individual abilities and interest of its pupils, teachers are generally teaching large, 30-person groups with generic courses. Yet, researchers have found that people dislike activities that are too easy or too hard [7], which means that this impersonal educational practice could lead to pupils switching off during classes. Secondly, the school system seems to be focused on prestige instead of failure: successful tests are rewarded with good grades, yet unsuccessful tests are considered a failure and are awarded low grades. Although trial and error is an extremely efficient learning concept, in the classroom, low grades are given for failed attempts, thereby discouraging students to keep trying. NASA did not reach space on its first shot, but kept trying and eventually landed a human being on the moon by learning from earlier "failed attempts". NASA's goals were reached not in spite of failing, but due to learning from failure [24].

These flaws in our educational system could be addressed with gamification strategies by making learning a more fun experience. For example, digital courses with gamified elements could tailor the classes to the individual students, despite group size. Also, additional courses could be added for those students who show particular interest in certain aspects. Furthermore, awarding students' progression with progress points after completing educational objectives assists them to track progress and encourage perseverance. Trial and error could be emphasized in gamified courses through awarding learning points, as an alternative for low grades, that could provide proof of a students' effort to reach a certain goal. I believe that institutions could improve the overall learning experience by adding gamification elements.

\section{GAMIFIED BUSINESSES}

Gamification could be used to boost business results as well. By implementing gamified elements like goals, achievements, rewards and leaderboards, gamified platforms could engage employees to more effectively track their work progress and subsequently promote action-taking behavior in order to succeed in business [25]. Employees that use gamified platforms may outperform others, with an increase return on investment as a result. A gamified cognitive training could more specifically increase the cognitive load limit of businessmen, for example by improving focus and thinking speed, thereby optimizing performance. 


\section{RECOMMENDATIONS FOR THE SERIOUS GAMES INDUSTRY}

While developing new treatments, creators in the industry can use our results to create games that are more suitable for elderly target groups, for which they can use our recommendations specifically tailored at PD patients, as presented in Chapter $\mathbf{3}$ and $\mathbf{4}$. Facilitators and barriers for treatment adherence should be addressed, such as patient-related, therapy-related, health care related, and disease-related factors. Another important consideration should be the introduction of a larger variety of training elements and levels in order to prevent boredom in the patient group. Moreover, a bottom-up design approach should be followed in which the industry, patients, researchers and caregivers collaborate to develop motivating therapies that can be executed in the home environment.

\section{TECHNOLOGICAL AND SOCIETAL READINESS}

The technology readiness level (TRL) scale was first used by NASA in the nineteen-seventies as systematic maturity assessment of a technology during its development, from idea generation to commercialization [26]. TRL is a nine-step ordinal scale (with 9 being the most mature) that is widely accepted as critical in making development decisions and investments for new technology. For good reason, it is used as a tool to evaluate both the potential and progression of European Horizon 2020 innovation projects [27]. Our gamified home-based cognitive intervention for PD has already completed TRL levels 1 to 4, which means that the technology concept has been formulated and the proof of concept have been developed and completed. We consider our technology to be at TRL 5 or 6 , indicating that the technology has been validated and demonstrated in a relevant population (PD) from a feasibility perspective, but the innovation still needs to be adjusted and efficacy needs to be proved in the real life setting.

Since the TRL focusses solely on the technological aspects of a project, the societal readiness level (SRL) was designed as an alternative scale to assess the societal adoption readiness of an innovation. The SRL is a nine-step ordinal scale and gives an impression of the steps required for a successful transition towards societal adaptation (as designed by Innovation Fund Denmark). SRL levels 1-3 reflect the work that was presented in this thesis, in which we included relevant stakeholders and target groups in the design process and we additionally identified the current base for gamified interventions in PD. SRL levels 4-6 have also been implicitly included in this thesis by forming and testing our research hypothesis as well as researching the impact of our product in the target group. SRL level 7 is the next step: our product needs to be refined and the subsequent results need to communicated to create awareness among relevant stakeholders in order to complete societal adaptation.

\section{COMMUNICATING RESEARCH TO THE PUBLIC}

Proper communication of scientific results to potential target groups is often overlooked in research. An essential requirement of a PhD program is the writing of scientific manuscripts to be published in scientific journals. This means that, with the correct search words and the right subscription, the results can be found by an academic audience through scientific search engines. Talks at specific conferences are also common ways of disseminating results to that 
audience. Research that is published in high impact journals may receive additional attention from regional or national news networks. When in luck, novel research will be picked up by the international press, which increases the likelihood of knowledge finding the appropriate audience. In addition to scientific publications, it is also common practice to translate scientific results into simpler language in order to inform the subjects that were included into studies. Nevertheless, scientific progress in cognitive training might be of interest to other audiences as well, such as caregivers (medical or psychological), businesses, the serious games industry, and the general public.

In line with the digital context of this thesis, I think it would be appropriate to follow a combined offline and online approach in communicating this research to the relevant stakeholders. Firstly, patients could be informed through patient associations via both newsletters and presentations. Secondly, a press release should be written as a short and appealing summary of the research. Thirdly, web-based, online media could be contacted in order to speak about this research in webinars and podcasts. Fourthly, conferences with a focus on eHealth, health care technology, and (neuro)psychology should be addressed and attended in order to reach an academic audience. Lastly, digital health tech blogs should be contacted to write a column about the investigated topic as well as the potential impact of the results.

Through these steps, I believe that we have a fair shot at conveying the prerequisites of gamification in home-based health care to relevant audiences, that can use this knowledge to their advantage in the future. 


\section{REFERENCES}

1. Boendermaker WJ, Gladwin TE, Peeters M, Prins PJM, Wiers RW. Training Working Memory in Adolescents Using Serious Game Elements: Pilot Randomized Controlled Trial. JMIR Serious Games JMIR Publications Inc., Toronto, Canada; 2018 May 23;6(2):e10. PMID: 29792294

2. Esposito F, Gendolla GHE, Van der Linden M. Are self-efficacy beliefs and subjective task demand related to apathy in aging? Aging \& Mental Health Routledge; 2014 Mar 12;18(4):521-530.

3. Medalia A, Saperstein A. The role of motivation for treatment success. Schizophr Bull 2011 Sep;37 Suppl 2(suppl 2):S122-8. PMID: 21860041

4. Jin J, Sklar GE, Min Sen Oh V, Chuen Li S. Factors affecting therapeutic compliance: A review from the patient's perspective. Ther Clin Risk Manag Dove Press; 2008 Feb;4(1):269-286. PMID: 18728716

5. Anderson M, Perrin A. Tech adoption climbs among older adults [Internet]. 2017 [cited 2020 Oct 19]. p. 1-23. Available from: https://www.pewresearch.org/internet/2017/05/17/tech-adoption-climbsamong-older-adults/

6. Wang S, Bolling K, Mao W, Reichstadt J, Jeste D, Kim H-C, et al. Technology to Support Aging in Place: Older Adults' Perspectives. Healthcare (Basel) Multidisciplinary Digital Publishing Institute; 2019 Apr 10;7(2):60. PMID: 30974780

7. Koster R. A Theory of Fun: for Game Design. 2nd ed. O’Reilly; 2013. p. 1-299.

8. Goldman JG, Litvan I. Mild cognitive impairment in Parkinson's disease. Minerva medica NIH Public Access; 2011;102(6):441.

9. Emre M, Aarsland D, Brown R, Burn DJ, Duyckaerts C, Mizuno Y, et al. Clinical diagnostic criteria for dementia associated with Parkinson's disease. Mov. Disord. 2007 Sep 15;22(12):1689-707-quiz 1837. PMID: 17542011

10. Gates NJ, Rutjes AW, Di Nisio M, Karim S, Chong L-Y, March E, et al. Computerised cognitive training for maintaining cognitive function in cognitively healthy people in late life. Cochrane Dementia and Cognitive Improvement Group, editor. Cochrane Database Syst Rev John Wiley \& Sons, Ltd; 2019 Mar 13;3(6):CD012277. PMID: 30864187

11. Goldman JG, Vernaleo BA, Camicioli R, Dahodwala N, Dobkin RD, Ellis T, et al. Cognitive impairment in Parkinson's disease: a report from a multidisciplinary symposium on unmet needs and future directions to maintain cognitive health. npj Parkinson's Disease 2018 Jun 26;4(1):608-11.

12. De Luca CR, Wood SJ, Anderson V, Buchanan J-A, Proffitt TM, Mahony K, et al. Normative data from the CANTAB. I: development of executive function over the lifespan. Journal of clinical and experimental neuropsychology 2003 Apr;25(2):242-254. PMID: 12754681

13. Committee on the Public Health Dimensions of Cognitive Aging, Board on Health Sciences Policy, Institute of Medicine, Blazer DG, Yaffe K, Liverman CT. Cognitive Aging: Progress in Understanding and Opportunities for Action. Washington (DC): National Academies Press (US); 2015 Jul 21. PMID: 25879131 
14. Perrot A, Maillot $P$, Hartley A. Cognitive Training Game Versus Action Videogame: Effects on Cognitive Functions in Older Adults. Games Health J Mary Ann Liebert, Inc., publishers 140 Huguenot Street, 3rd Floor New Rochelle, NY 10801 USA; 2019 Feb;8(1):35-40. PMID: 30376364

15. Perrochon A, Borel B, Istrate D, Compagnat M, Daviet J-C. Exercise-based games interventions at home in individuals with a neurological disease: A systematic review and meta-analysis. Ann Phys Rehabil Med 2019 May 9;62(5):366-378. PMID: 31078706

16. van der Kolk NM, de Vries NM, Kessels RPC, Joosten H, Zwinderman AH, Post B, et al. Effectiveness of home-based and remotely supervised aerobic exercise in Parkinson's disease: a double-blind, randomised controlled trial. The Lancet Neurology 2019 Nov;18(11):998-1008. PMID: 31521532

17. Smith PJ, Blumenthal JA, Hoffman BM, Cooper H, Strauman TA, Welsh-Bohmer K, et al. Aerobic exercise and neurocognitive performance: a meta-analytic review of randomized controlled trials. Psychosom Med 2010 Apr;72(3):239-252. PMID: 20223924

18. Walton CC, Shine JM, Mowszowski L, Naismith SL, Lewis SJG. Freezing of gait in Parkinson's disease: current treatments and the potential role for cognitive training. Restor. Neurol. Neurosci. 2014;32(3):411-422. PMID: 24531294

19. Garcia-Agundez A, Folkerts A-K, Konrad R, Caserman P, Tregel T, Goosses M, et al. Recent advances in rehabilitation for Parkinson's Disease with Exergames: A Systematic Review. J Neuroeng Rehabil 2019 Jan 29;16(1):17. PMID: 30696453

20. PhD DAM, PhD PLR, PhD IM, Del Din PhD S, PhD LA, MS FN, et al. Articles Addition of a non-immersive virtual reality component to treadmill training to reduce fall risk in older adults (V-TIME): a randomised controlled trial. Lancet Elsevier Ltd; 2016 Aug 11;388(10050):1-13.

21. Bloem BR, Marks WJ, Silva de Lima AL, Kuijf ML, Van Laar T, Jacobs BPF, et al. The Personalized Parkinson Project: examining disease progression through broad biomarkers in early Parkinson's disease. BMC Neurol BioMed Central; 2019 Jul 17;19(1):160-10. PMID: 31315608

22. Roy S, Jha R, Karnes A, Oates P, Wojciechowski K, Dvorak A, et al. Innovative eHealth at Home Program Reduces Hospitalization and Readmission Rates of Patients with Heart Failure. Journal of Cardiac Failure Elsevier B.V; 2019 Aug 1;25(Supplement):S127.

23. Suppan L, Abbas M, Catho G, Stuby L, Regard S, Harbarth S, et al. Impact of a Serious Game on the Intention to Change Infection Prevention and Control Practices in Nursing Homes During the COVID-19 Pandemic: Protocol for a Web-Based Randomized Controlled Trial. JMIR Res Protoc 2020;9(12):e25595-12.

24. Madsen PM, Desai V. Failing to Learn? The effects of failure and success on organizational learning in the global orbital launch vehicle industry. Academy of Management Journal 2010 Jun 18;53(3):451-476.

25. Klevers M, Sailer M, Günthner WA. Implementation Model for the Gamification of Business Processes: A Study from the Field of Material Handling. In: Kaneda T, Kanegae H, Toyoda Y, Rizzi P, editors. Simulation and Gaming in the Network Society Singapore: Springer Singapore; 2016. p. 173-184.

26. Conrow EH. Estimating Technology Readiness Level Coefficients. Journal of Spacecraft and Rockets 2011 Jan;48(1):146-152.

27. Héder M. From NASA to EU: the evolution of the TRL scale in Public Sector Innovation. The Public Sector Innovation Journal 2017 Sep 1;22(2):1-23. 


\section{DANKWOORD}

Het spreekwoord 'vele handen maken licht werk' is zeker van toepassing op mijn proefschrift. Zonder hulp en ondersteuning van belangrijke anderen was het mijns inziens onmogelijk geweest om mijn proefschrift af te ronden. De mensen die voor mij van onmisbare waarde zijn gebleken, wil ik in dit dankwoord in het zonnetje zetten.

Allereerst gaat mijn dank uit naar mijn promotieteam. Toen dr. Annelien Duits en dr. Mark Kuijf mij benaderden om als promovendus te komen werken en dit project te leiden, vond ik dat een hele eer. Jullie verschillende expertises (neuropsychologisch en neurologisch) combineerden prachtig in dit project en ik heb telkens energie geput uit jullie enthousiasme. Jullie spraken jullie vertrouwen in mij uit en hebben me de vrijheid gegeven om mezelf en dit project te ontwikkelen, wat uiteindelijk niet alleen geresulteerd heeft in dit proefschrift, maar ook in een ontwikkeling van mijn vaardigheden. Annelien, je was (en bent nog steeds) een luisterend oor en altijd in voor een babbel. De kans om dit project te mogen draaien komt uit jouw koker, heeft mij als persoon ontwikkeld, en daar ben ik je dankbaar voor. Mark, als mede-projectleider ben ik je dankbaar voor je begeleiding tijdens mijn eerste stappen in de onderzoekswereld en voor je sterke inhoudelijke medische bijdrage in mijn publicaties. Dat heeft een lichtje aangewakkerd! Dr. Nienke de Vries is gedurende de loop van het project toegetreden tot mijn promotieteam. Sindsdien heb ik enorm veel projectmatige en organisatorische kennis opgedaan van deze kanjer en ik heb tevens heel veel geleerd van je intelligente schrijfstijl. Het team in Nijmegen mag heel blij zijn met jou als hoofdonderzoeker en onderzoeksmanager. Prof. dr. Bas Bloem is de inspirator en najager van dit project. Als promotor heb jij me telkens omringd met kwalitatieve collega's die het project naar een hoger niveau konden tillen. Zakelijk gezien heb ik bewondering voor je onuitputtelijke positivisme, het altijd op zoek zijn naar meer, en het verbinden van expertises. Persoonlijk gezien ben ik je dankbaar voor het opvangen van mijn promotietraject op de momenten dat de voortzetting in het gedrang kwam. Ik hoop dat de toekomst nog mooie samenwerkingen in het verschiet zal hebben.

Hartelijk dank aan de leden van de beoordelingscommissie, prof. dr. S.A. Kotz (voorzitter), prof. dr. C.M. van Heugten, prof. dr. L. Fasotti en dr. R. Esselink, voor jullie tijd en moeite om dit proefschrift te lezen en beoordelen.

Dear late Keiron Sparrowhawk, thank you for your trust in the abilities of our team in the Netherlands. As an entrepreneur, your friendly leadership-style to the MyCognition company and your endless optimism were super inspiring for me: it made me see that perseverance wins in the end. In addition, your warmth made me feel at home immediately during my frequent visits to London. It is soothing to know that our scientific publication on your beloved product came in time for you. May you rest in peace. My thoughts are with Imogen, your family, and friends.

Thank you Duncan Knight, for the fun “business” trips to Aachen and other cities. I still can't believe that bananabeer is just regular bear with banana-flavoured lemonade, can you?! 
Anna Domen, mijn MyCognition-research-counterpart in Amsterdam. Jij voerde een soortgelijk onderzoek in een andere doelgroep uit, en dat hielp mij enorm op weg om met het videogame platform te werken. Ik heb er bewondering voor dat jij je eigen pad hebt durven kiezen, en tevens ben ik dankbaar voor je inspanningen voor het MyCQ validatie artikel.

Het gros van mijn promotietraject heb ik doorgebracht op de onderzoekskamer op de stafgang Neurologie in Maastricht. De mensen die ik daar het meeste sprak en zag waren Anne-France, Amir, Bianca, Daniëlle, Eefje, Eleana, Eric, Irene, Isis, Maurice, Renske, en Sara. In een groep werken zorgt ervoor dat je de lasten kunt delen, en in mijn geval profiteerde ik van jullie kennis op het gebied van promoveren. Bovendien hebben jullie meer dan eens telefoontjes van nieuwe deelnemers voor mij aangenomen (daar waren jullie overigens ook erg goed in). Ik denk met plezier terug aan de leuke uitjes, de reis naar Barcelona, de tosti-middagen, en natuurlijk de enorm effectieve pomodoro's.

Voor het uitvoeren van onderzoek ben je afhankelijk van verschillende personen die je helpen. Tijdens mijn promotietraject hebben verschillende uitzonderlijke talenten mij geholpen met het verzamelen en analyseren van data. Ashley, Joni, Amée en Vienna: elk hebben jullie op jullie eigen manier een waardevolle bijdrage geleverd. Niet alleen in het zien van patiënten, maar ook in het plannen en registreren waren jullie onmisbaar. Hoewel dit proefschrift voornamelijk betrekking heeft op het onderzoek in Maastricht, wil ik mijn dank uitspreken aan Eline, Arjonne, Ilse en Berber voor hun hulp in Nijmegen. Jullie stelden mij in staat om aandacht te schenken aan de projecten die ten grondslag liggen aan dit proefschrift. Voor zowel de dames in Maastricht als in Nijmegen geldt: het uitvoeren van dit onderzoek was zeker niet zo gezellig geweest zonder jullie.

Ook wil ik Mirella Davies-Waber (verpleegkundig specialist Parkinson in Maastricht) danken voor haar hulp bij de vele metingen met patiënten. Jouw expertise met bewegingsstoornissen heeft enorm geholpen in de snelle UPDRS metingen. Jij hebt hart voor patienten en helpt hen dag in dag uit om een betere kwaliteit van leven te krijgen. Verschillende technici hebben verder ondersteund in het inrichten van randzaken voor mijn onderzoek, waaronder Nico, Ron, maar ook de verschillende collega's bij Radiologie (fMRI). Dank jullie wel voor jullie inzet.

Het werven van deelnemers is essentieel bij medisch wetenschappelijk onderzoek, en in dat kader hebben Gerrit en Tineke (Zuyderland Heerlen), Jessica en Nienke (Radboudumc Nijmegen) en de Neurologen in Maastricht, onder leiding van prof. dr. R.J. van Oostenbrugge, een enorme bijdrage geleverd. Ook spreek ik een speciaal woord van dank uit naar twee online platformen die geholpen hebben in (snelle) digitale werving van deelnemers, namelijk de Parkinson Vereniging en ParkinsonNext. In de laatste plaats spreek ik mijn dank uit aan alle patiënten die tijd hebben gemaakt om deel te nemen aan deze onderzoeken. Zonder jullie kunnen wij als wetenschappers geen onderzoek uitvoeren. 
Daarnaast wil ik Anoek Meijers-Erdkamp bedanken voor de (voor haar vermoedelijk onbewuste) bijdrage die zij geleverd heeft in de totstandkoming van dit proefschrift. Als collega's werkten we nauw samen binnen een digitaal zorgproject, en dankzij jouw collegialiteit en flexibiliteit stelde je mij in staat om resterende taken uit te voeren die nodig waren om dit proefschrift af te ronden. Ik kijk met plezier terug op onze samenwerking.

Tijdens de afronding van mijn proefschrift ben ik bij het Centrum voor Acute en Kritieke Zorg gaan werken als stafadviseur Informatiemanagement en Onderzoek (MUMC+). In het bijzonder mijn collega's/werkgevers Freek van Daal, Wolfgang Buhre en Iwan van der Horst hebben mij daar laten inzien dat - zélfs in een grote organisatie - zorg ook op innovatieve manieren geboden kan worden, zolang je maar in kansen denkt en vertrouwen uit straalt. Het jaar 2020 en 2021 is een enorm leerzaam jaar gebleken. Dank jullie wel voor jullie vertrouwen in mij, en ik kijk uit naar de projecten die we nog samen gaan opzetten: op naar World Peace!

In Sittard en Maastricht hebben vele vrienden mij elk op hun eigen manier geholpen om mijn (onderzoeks)werk goed te kunnen doen. In het bijzonder wil ik een woord van dank uitspreken naar mijn paranimfen, die mij tijdens de verdediging van mijn proefschrift zullen bijstaan. Raoul: we zijn al tientallen jaren vrienden. Ik hoef je maar voor hulp te vragen en je staat voor me klaar. We kunnen altijd goede gesprekken voeren, meestal over iets en soms ook over niets. Je humor is verlichtend, al is hij vaak ook herhalend. Ik vind het heel tof dat je me gaat bijstaan tijdens de verdediging, en ben benieuwd naar het feest dat je voor mij gaat organiseren. Tom: ik wil jou bedanken voor je oprechte en frequente interesse in mijn proefschrift en in mijn dagelijkse functioneren. Het mooie aan onze vriendschap vind ik dat we het leven samen kunnen vieren met iets eenvoudigs als een lekker biertje. Toch bied je ook een luisterend oor als het een keer wat moeilijker gaat. Ik kijk op tegen jouw creatieve doorzettingsvermogen in je werk en de rust die je uit kan stralen, en benijd ik tevens in onze vriendschap. Ik hoop dat mijn vriendschap met jullie beide nog lang voort zal duren.

Ook mijn familieleden hebben mij in staat gesteld om dit proefschrift tot een goed einde te brengen. In het bijzonder wil ik mijn lieve ouders bedanken voor hun steun tijdens mijn dynamische carrière. Jullie stimuleren me om na te streven waar ik gelukkig van word, omdat jullie zien waar ik energie van krijg. Jullie stellen kritische vragen, die me laten nadenken over het pad dat ik moet bewandelen. Jullie spreken vertrouwen uit en staan voor me klaar, altijd. Ik ben trots op jullie.

Hoewel menigeen niet zo positief zal spreken over zijn schoonouders, is dat in mijn geval anders. Hans en Anja, jullie stimuleren me om mezelf te zijn en naar mijn waardes te leven. Jullie tonen begrip voor mijn dynamische werk en proberen daar rekening mee te houden. Dank jullie wel voor jullie steun.

Lieve Paulien, naast elke "succesvolle" man staat een sterkere vrouw. Het is onmogelijk om mijn ambities na te streven zonder jou. Je stelt de juiste vragen, op het juiste moment. Jij 
bouwt mijn structuur. Jij creëert ruimte om mij heen, met bewonderenswaardige flexibiliteit wanneer ik weer "even iets moet doen voor werk". Je bent de meest liefdevolle partner en de liefste moeder. Door jou kan ik mijn dromen waarmaken, waar jij er één van bent. Ik ben er enorm trots op dat jij aan mijn zijde staat en kijk uit naar onze toekomst samen. 


\section{ABOUT THE AUTHOR}

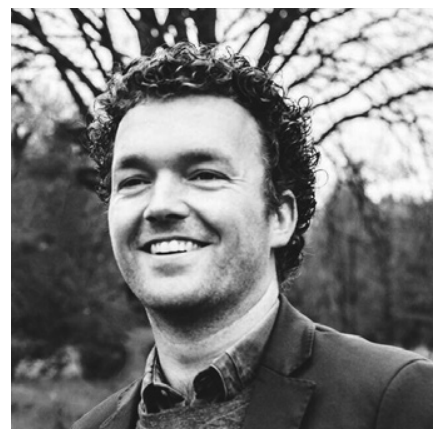

English: Sjors Camille Frans van de Weijer was born on the $12^{\text {th }}$ of February 1988 in Maastricht, the Netherlands. He graduated in 2005 from highschool (HAVO, Porta Mosana College Maastricht). In the same year, he started studying Applied Psychology at Fontys Eindhoven. The following year, after receiving a propaedeutic transcript, he started studying Health Sciences at Maastricht University. In 2007, he co-founded his first company AUDIOEYE. Whilst obtaining his Bachelor's degree in Psychology (2010) and Master's degree in Health Sciences (2011), he built AUDIOEYE into an acknowledged event technology rental and sales firm in the southern part of the Netherlands. In 2012, he co-founded a software company in the health care space, which ultimately led to co-founding Clementine: a health care technology company focusing on creating awareness for hearing impairment and enhancing the connection between audiology services and potential clients.

In 2015, Sjors started working as a PhD student at the Neurology Department of the Maastricht University Medical Center (MUMC+), he later continued his PhD work at Radboudumc Nijmegen and wrote a PhD thesis on digital technology-based home health care. Sjors was nominated for the Upcoming Scientist Award in 2017 (Innovation for Health) and the Hans Lakke Prize in 2020 (Parkinson Vereniging) for his work in the Parkin'Play Study.

In his carreer, Sjors combines his passion for technology, health care, and research, and through his work, he gained extensive experience in these areas. Lately, in an advisory position on information management \& innovation research for the Centre for Acute and Critical Care at MUMC+, he has been overseeing the Emergency Department, Operation Rooms, and Medium and Intensive Care Units. Sjors is currently working as Manager Research and Development at the Hearing Screening department at Eargo Inc. (San Jose, CA, USA). 
Nederlands: Sjors Camille Frans van de Weijer is op 12 februari 1988 geboren in Maastricht (Nederland). Hij verkreeg zijn HAVO-dimploma in 2005 (Porta Mosana College Maastricht). In datzelfde jaar startte hij met de opleiding Toegepaste Psychologie aan Fontys Eindhoven, waarhij zijn propedeuse haalde. Het daaropvolgende jaar begon hij aan de opleiding Gezondheidswetenschappen aan Universiteit Maastricht. In 2007 richtte hij zijn eerste bedrijf AUDIOEYE op. Terwijl hij een Bachelor Psychologie (2010) en een Master Gezondheidswetenschappen (2011) voltooide, bouwde hij AUDIOEYE uit tot een gerenomeerd verhuurbedrijf in de evenementenindustrie in het zuiden van Nederland. In 2012 richte hij een softwarebedrijf op dat zich focuste op de gezondheidszorg, dat uiteindelijk leidde tot het mede-oprichten van Clementine: een gezondheidszorg technologiebedrijf dat zich richt op het vergroten van bewustzijn voor hoorproblematiek en het faciliteren van de verbinding tussen hoordiensten en potentiële klanten.

Sjors startte in 2015 met een PhD op de afdeling Neurologie van het Maastricht Universitair Medisch Centrum, werkte later aan het Radboudumc Nijmegen en schreef een proefschrift over digitale en technologische zorg aan huis. Sjors werd genomineerd voor de Upcoming Scientist Award in 2017 (Innovation for Health) en de Hans Lakke Prijs in 2020 (Parkison Vereniging) voor zijn promotie-onderzoek.

In zijn carriere combineert Sjors zijn passie voor technologie, gezondheid(szorg) en onderzoek. Als stafadviseur Informatiemanagement en Onderzoek voor het Centrum voor Acute en Kritieke Zorg (MUMC+) overziet hij informatie- en onderzoeksvraagstukken voor de afdelingen Spoed-Eisende-Hulp, Operatiekamers en Intensive Care. Sjors werkt momenteel als Manager Research and Development op de afdeling Hoorscreening bij Eargo Inc. (San José, Californië, VS). 
190 APPENDICES 


\section{LIST OF PUBLICATIONS}

1. Van de Weijer SCF, Duits AA, Bloem BR, Kessels RP, Jansen JFA, Köhler S, Tissingh G, Kuijf ML. The Parkin'Play study: protocol of a phase II randomized controlled trial to assess the effects of a health game on cognition in Parkinson's disease. BMC Neurol. BioMed Central; 2016 Nov 3;16(1):209. PMCID: PMC5093943

2. Van de Weijer SCF, Duits AA, Bloem BR, Kessels RP, Jansen JFA, Köhler S, Tissingh G, Kuijf ML. A versatile web-based training: to slow down cognitive decline in Parkinson's disease. Poster presented at: Innovation for Health Conference 2017; 2017 Feb 16; Rotterdam.

3. Van de Weijer SCF, Hommel ALAJ, Bloem BR, Nonnekes J, De Vries NM. Promising non-pharmacological therapies in PD: Targeting late stage disease and the role of computer based cognitive training. Parkinsonism and Related Disorders 2018 Jan;46 Suppl 1:S42-S46. PMID: 28923292

4. Wolters AF, van de Weijer SCF, Leentjens AFG, Duits AA, Jacobs HIL, Kuijf ML. Resting-state fMRI in Parkinson's disease patients with cognitive impairment: A meta-analysis. Parkinsonism and Related Disorders 2019 May;62:16-27. PMID: 30580907

5. Domen AC, van de Weijer SCF, Jaspers MW, Denys D, Nieman DH. The validation of a new online cognitive assessment tool: The MyCognition Quotient. Int J Methods Psychiatr Res. John Wiley \& Sons, Ltd; 2019 Feb 13;6(10):e1775.

6. Van de Weijer SCF, Kuijf ML, de Vries NM, Bloem BR, Duits AA. Do-It-Yourself Gamified Cognitive Training: Viewpoint. JMIR Serious Games. 2019 May 7;7(2):e12130.

7. Nuic D, van de Weijer SCF, Olivier C, Cherif S, Foulon P, Bloem BR, de Vries NM, Welter ML. Rééducation par les serious games des troubles de la marche et de l'équilibre chez les patients avec maladie de Parkinson : étude randomisée contrôlée à domicile. Neurophysiologie Clinique / Clinical Neurophysiology Elsevier Masson SAS; 2019 Dec 1;49(6):429.

8. De Joode LEGH, Martin EC, Stultiens JJA, Leue C, Delespaul P, Peeters F, Erdkamp A, van de Weijer SCF, Blom H, Bruintjes T, Zwergal A, Grill E, Guinand N, Perez-Fornos A, van de Berg MR, Widdershoven J, Kingma H, van de Berg R. The DizzyQuest: to have or not to have... a vertigo attack? Journal of Neurology; 2020 Dec;267(6):15-23. PMID: 32654061

9. Martin EC, Leue C, Delespaul P, Peeters F, Janssen AML, Lousberg R, Erdkamp A, van de Weijer SCF, Widdershoven J, Blom H, Bruintjes T, Zwergal A, Grill E, Guinand N, Perez-Fornos A, van de Berg MR, Stultiens JJA, Kingma H, van de Berg R. Introducing the DizzyQuest: an app-based diary for vestibular disorders. Journal of Neurology; Springer Berlin Heidelberg; 2020 Jul 25;9(6):1076-12. PMID: 32712867

10. Van de Weijer SCF, Duits AA, Bloem BR, de Vries NM, Kessels RPC, Köhler S, Tissingh G, Kuijf ML. Feasibility of a Cognitive Training Game in Parkinson's Disease: The Randomized Parkin'Play Study. Eur Neurol Karger Publishers; 2020 Aug 5;83(3):1-7.

11. Koelmans BEA, Verwey NA, van de Weijer SCF, Bloem BR, Nonnekes J. Tailored use of compensation strategies to enable exercise despite festination in parkinson's disease. J Rehabil Med Clin Commun 2021;4:1000055. PMID: 34276902 

This thesis investigates important aspects of gamification in home-based cognitive training. It analyzes the adherence to such interventions in persons with Parkinson's disease by outlining the current knowledge on cognitive training and cognitive assessments, by researching the biological basis of cognitive decline, by gauging the interest level for gamified therapies, and by presenting the results of a gamified cognitive training study. These answers could help to establish new home-based, personalized cognitive treatment programs that incorporate attractive elements to promote long-term cognitive effects. 